University of Louisville

ThinkIR: The University of Louisville's Institutional Repository

Electronic Theses and Dissertations

$12-2014$

\title{
Evaluating the effectiveness and hydrological performance of green infrastructure stormwater control measures.
}

Hamidreza Kazemi

University of Louisville

Follow this and additional works at: https://ir.library.louisville.edu/etd

Part of the Civil and Environmental Engineering Commons

\section{Recommended Citation}

Kazemi, Hamidreza, "Evaluating the effectiveness and hydrological performance of green infrastructure stormwater control measures." (2014). Electronic Theses and Dissertations. Paper 1744.

https://doi.org/10.18297/etd/1744

This Doctoral Dissertation is brought to you for free and open access by ThinkIR: The University of Louisville's Institutional Repository. It has been accepted for inclusion in Electronic Theses and Dissertations by an authorized administrator of ThinkIR: The University of Louisville's Institutional Repository. This title appears here courtesy of the author, who has retained all other copyrights. For more information, please contact thinkir@louisville.edu. 


\title{
EVALUATING THE EFFECTIVENESS AND HYDROLOGICAL PERFORMANCE OF GREEN INFRASTRUCTURE STORMWATER CONTROL MEASURES
}

\author{
by \\ Hamidreza Kazemi

\begin{abstract}
A Dissertation
Submitted to the Faculty of the

J.B. Speed School of Engineering of the University of Louisville

in Partial Fulfillment of the Requirements

for the Degree of
\end{abstract}

Doctor of Philosophy

Department of Civil and Environmental Engineering

University of Louisville

Louisville, Kentucky

December 2014 
(C) Copyright 2014 by Hamidreza Kazemi

All rights reserved 



\title{
EVALUATING THE EFFECTIVENESS AND HYDROLOGICAL PERFORMANCE OFGREEN INFRASTRUCTURE STORMWATER CONTROL MEASURES
}

\author{
Submitted by: Hamidreza Kazemi
}

A dissertation approved on

December $5^{\text {th }}, 2014$

by the following dissertation committee:

Dr. Thomas Rockaway, Dissertation Director

Dr. J.P. Mohsen

Dr. Mark McGinley

Dr. William Biles 


\section{DEDICATION}

To my loving parents and family who have supported and encouraged me through all years of my life.

And to my beautiful Parvaneh, who has filled my life with love and passion, and stood by me patiently during the completion of this work. 


\section{ACKNOWLEDGMENTS}

This document wouldn't have been completed without the support and wisdom of my advisor and director of Center for Infrastructure Research, Dr. Thomas Rockaway. I'm grateful for his insight and support during all years of my education in University of Louisville. His encouragement and indispensable guidance led me to the completion of this research. Dr. Rockaway has been and will always be a great mentor and role model.

I'd also like to thank Joshua Rivard, a great friend who made me feel welcomed in Center for Infrastructure Research and has provided me with numerous guidance and recommendations through all my endeavors.

I'd like to express my gratitude to my doctoral defense committee members, Dr. Mark McGinley, Dr J.P. Mohsen, and Dr. William Biles, for sharing their experiences and insightful observations and suggestions.

Finally I would also like to acknowledge the wonderful people of USEPA Office of Research and Development at Edison, NJ, especially Michael Borst and Dr. Robert A. Brown who made my stay a unique and priceless learning experience and have provided me with many valuable advices during this research. 


\title{
ABSTRACT \\ EVALUATING THE EFFECTIVENESS AND HYDROLOGICAL PERFORMANCE OF GREEN INFRASTRUCTURE STORMWATER CONTROL MEASURES
}

\author{
Hamidreza Kazemi
}

December 5, 2014

The Louisville and Jefferson County Metropolitan Sewer District (MSD) has developed a Long-Term Control Plan (LTCP) to address the combined sewer overflow (CSO) issue through a combination of gray and green infrastructure (GI) stormwater management practices in the city of Louisville. In support of this effort, the MSD installed a series of GI stormwater control measures (SCMs) to abate the CSO volumes in an urban sewershed basin. This research ia focused on monitoring and evaluating the individual hydrological performance, which included infiltration and exfiltration processes, of two permeable pavement systems over a two year study period. It also assess the combined effectiveness of the suite of GI SCMs in achieving the CSO mitigation.

Assessing the hydrological performance of the permeable pavement systems was completed through collected data from embedded electronic sensors and field measurements. Modeling techniques were employed to predict the changes of water level within the storage layer under pavement sections. The developed model was used as an assessment tool to monitor the hydrological performance of the two GI controls. Ultimately 
the results from the developed model and other data analysis techniques were used to track changes in infiltration and exfiltration performances of both permeable pavement systems.

The infiltration performance was observed to be a key component affecting the hydrological performance of a permeable pavement system. The infiltration capacity is limited by clogging formed on the surface of the permeable pavement section but can be restored after applying a suitable maintenance treatment.

The modeling effort also provided understanding of the exfiltration processes as the GI controls transferred the captured stormwater runoff to underlying and surrounding soil layers. Seasonal changes in system performance were observed and attributed to changes in dynamic viscosity of water caused by variation of temperature. It was also observed that exfiltration performance is affected by infiltration capacity of the system.

To assess the effectiveness of the GI controls in achieving the CSO mitigation, a regression analysis modelling effort was conducted to compare the pre and postconstruction conditions. The results showed that almost 2.6 million gallons of overflow volumes were eliminated from the combined sewer system during the second half of 2013 and following the construction of all GI stormwater controls. 


\section{TABLE OF CONTENTS}

DEDICATION ...................................................................................

ACKNOWLEDGMENTS ............................................................. iv

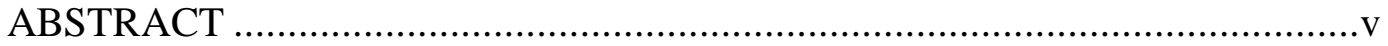

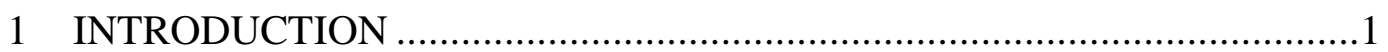

$1.1 \quad$ Background ..........................................................................

1.2 Statement of the Problem ........................................................

1.3 Objectives of this Research ...................................................6

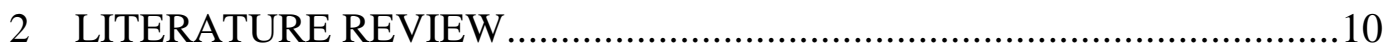

2.1 Introduction ....................................................................... 10

2.2 Combined Sewer Systems .......................................................... 11

2.3 Effects of Urbanization on the Hydrological Cycle .............................14

2.4 Green Infrastructure Stormwater Control Measures............................19

2.4.1 Stormwater Volume Control ..................................................21

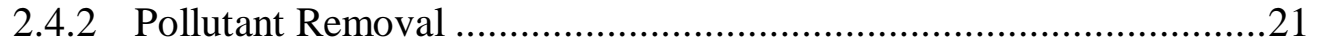

2.4.3 Water Conservation ...........................................................22

2.4.4 Non-Water Benefits ..............................................................22 
2.5 Different Types of GI Stormwater Controls

2.6 Permeable Pavement Systems ........................................................26

2.7 Hydrological Performance of Permeable Pavement Systems ................29

2.7.1 Infiltration Capacity and Surface Clogging ................................29

2.7.2 Surface Maintenance Treatments .............................................35

2.7.3 Exfiltration Performance and Contributing Factors .......................37

2.7.4 Rainfall Characteristics of Storm Events .................................. 41

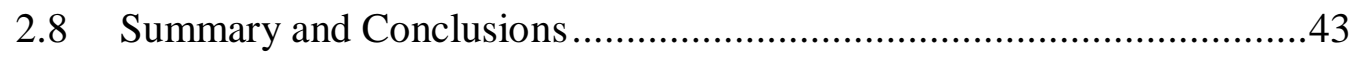

3 EXPERIMENTAL APPROACH ….................................................45

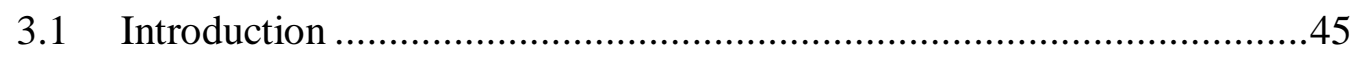

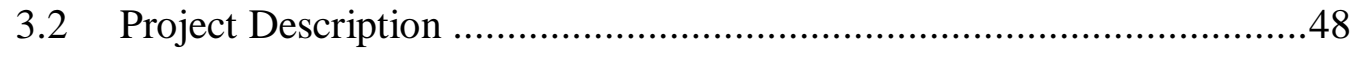

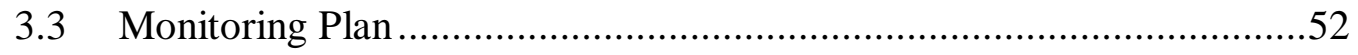

3.3.1 Instrumentation.............................................................53

3.3.1 One-Time Measurements ...................................................59

3.3.2 Measurements Conducted Periodically ....................................65

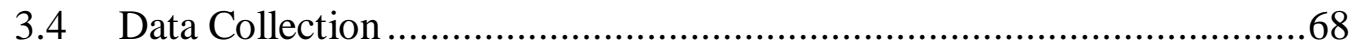

3.4.1 Data/Sampling Frequency ...............................................6 69

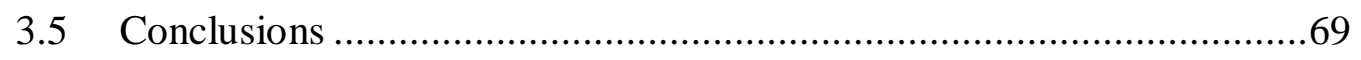

4 ELECTRONIC DATA ANALYSIS AND MODELING ..........................71

$4.1 \quad$ Rainfall Data........................................................................... 71 
4.1.1 Rainfall Intensities

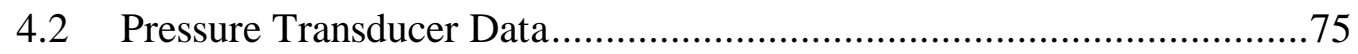

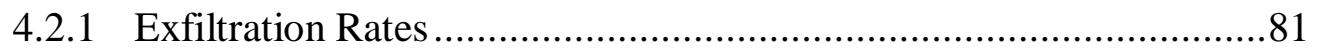

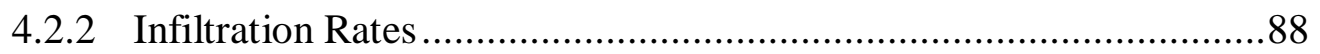

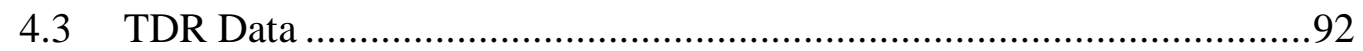

4.3.1 Surface Clogging Progression Rate ……………............................97

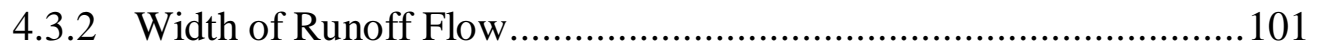

4.4 Changes in Exfiltration Rates and Contributing Factors ........................104

5 MODELING THE WATER LEVEL INSIDE GI CONTROLS ...................123

5.1 Modeling Technique 1: One Variable Exfiltration Rate ........................127

5.2 Modeling Technique 2: Multiple Constant Exfiltration Rates ...............130

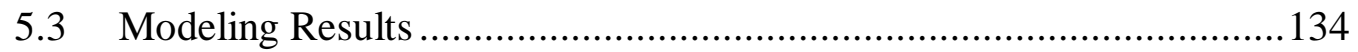

5.4 Captured Stormwater Volume .............................................................. 141

6 CHANGES IN INFILTRATION CAPACITY AND SURFACE

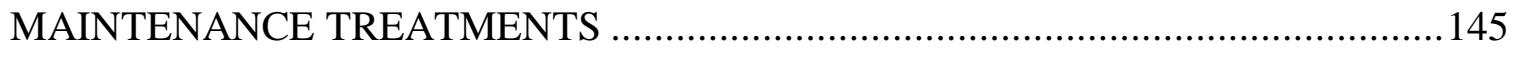

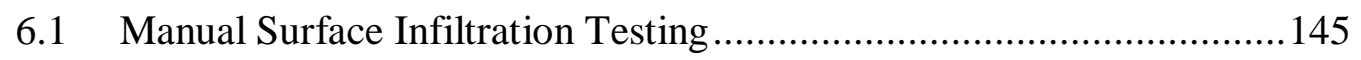

6.2 Maintenance Treatment Methods and their Effectiveness .....................148

6.2.1 Surface Infiltration Test Results ...................................................153

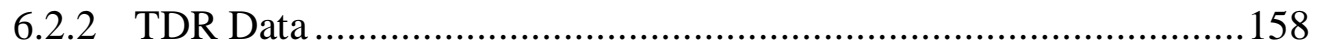

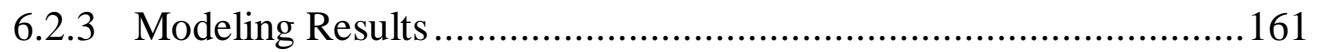


6.3.1 Size Distribution .164

6.3.2 Organic Content. 168

7 OVERALL EEFFECTIVENESS OF GI STORMWATER CONTROLS ...170

7.1.1 Modeling the Overflows of CSO130. 172

7.1.2 Effectiveness of GI Controls 180

8 DISCUSSION OF THE RESULTS. 183

8.1 Infiltration Capacity. 184

8.2 Exfiltration Performance

8.3 Effectiveness of GI Controls in CSO Mitigation. 191

9 CONCLUSIONS AND FUTURE WORK .194

9.1 Conclusions .194

9.2 Future Work

REFERENCES 199

I. APPENDIX: SENSOR NAMING. .208

II. APPENDIX: DETERMINATION OF ATTACHED SOLIDS . .212

III. APPENDIX: SURFACE INFILTRATION TESTS .221

IV. WATER LEVEL CHANGES IN GI CONTROLS 19G AND 19H .........224

CURRICULUM VITAE .228 


\section{LIST OF FIGURES}

Figure 1 - Combined (top) and Separate (down) Sewer Systems (U.S.EPA 2004) ..........12

Figure 2 - Communities with Combined Sewer Systems (U.S.EPA 2012b) ..................13

Figure 3 - Water Cycle between Land, Ocean, and Atmosphere (Winter et al. 1998)......15

Figure 4 - Relationship between Impervious Cover and Surface Runoff (U.S.EPA 2003)

Figure 5 - Relationship between Imperviousness Coverage and Stream Health (Arnold

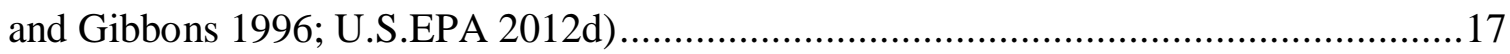

Figure 6 - Relationship between Watershed Imperviousness and Runoff Coefficient (Schueler 1994).

Figure 7 - An Application of Permeable Interlocking Concrete Paver in Louisville, KY 28 Figure 8 - Example of a rainfall hyetograph (a), and cumulative rainfall depth (b) (Pazwash 2011) 42

Figure 9 - Discharge Point of CSO130 to Beargrass Creek (Badwaterjournal.com 2010)

Figure 10 - Proposed GI controls for CSO130...............................................47

Figure 11 - Dimension and Setup of Articluated Concrete Pavers (PaveDrain 2011) ......49

Figure 12 - Cross Sections of GI Controls 19G (left) and 19H (right) ..........................50

Figure 13 - Construction Stages of Controls 19G \& 19H......................................51 
Figure 14 - TDR, Campbell Scientific Model CS650 (image source: Campbell Scientific Co.) .54

Figure 15 - Plan View of TDRs Placement at the Interface of \#3 and \#57 Stone Layers .54 Figure 16 - Pressure Transducer, Campbell Scientific Model CS450 (image source: Campbell Scientific Co.) .55

Figure 17 - Location of Pressure Transducers along the length of each GI Control.........56 Figure 18 - Location of Different Sensors along the Depth of GI Control......................57

Figure 19 - Installing Sensors in Controls 19G \& 19H ............................................60 Figure 20 - Water Level versus Time for Falling Head Tests, Controls 19G and 19H....62 Figure 21 - Porosity Measurements during Construction of Controls 19G \& 19H .........64 Figure 22 - Surface Infiltration Test Apparatus (Borst et al. 2010) .............................67 Figure 23 - Comparison of Annual Cumulative Rain for Years 2012, 2013 (Study Period) and 2001 (Design Year) .73

Figure 24 - Monthy Rainfall Depths for Years 2012, 2013 (Study Period) and 2001

(Design Year)

Figure 25 - Maximum 5-minute Intensity Values Versus Maximum 15-minute Intesity

Values .75

Figure 26 - Changes in Captured Water Level, Control 19G . .76

Figure 27 - Changes in Captured Water Level, Control 19H .77

Figure 28 - Differences between Measured Water Levels from the three Pressure Transducers, Control 19G .78

Figure 29 - Developed Gradient in the GI Control as Surface Clogging Advances .........79

Figure 30 - Developed Gradient in Control 19G Following its Construction 80 
Figure 31 - Developed Gradient in Control 19G before and after a Successful Maintenance .81

Figure 32 - Changes of Recession Rates versus the Water Depth, Control 19G..... .83

Figure 33 - Changes of Recession Rates versus the Water Depth, Control 19H. .84

Figure 34 - Mean Recession Rates for Controls 19G and 19H for the first Five Rainfall

Events .85

Figure 35 - Exfiiltration Rates for Controls $19 \mathrm{G}$ and 19H for the first Five Rainfall

Events

Figure 36 - An equation Can Be Established for Estimating the Exfiltration Rates based on the Water Depth inside the Trench .....

Figure 37 - Intra-Event Exfiltration for Controls 19G and 19H .88

Figure 38 - Escalation Rates versus Average 15-min Intensities for Controls 19G and $19 \mathrm{H}$ .90

Figure 39 - Infiltration Rates versus Average 15-min Intensities for Controls 19G and $19 \mathrm{H}$ .92

Figure 40 - Curbside TDR Responses for the First Four Rainfall Events, Control 19G...95 Figure 41 - Maximum Recorded VWCs by Curbside TDRs versus Time (Event Number), Control 19G. .96

Figure 42 - Maximum TDRs’ Responses along Curbside versus Cumulative Rainfall, Control 19G

Figure 43 - Maximum TDRs’ Responses along Curbside versus Cumulative Rainfall,

Control $19 \mathrm{H}$ .98

Figure 44 - Selecting a Threshold for Determining the Surface Clogging Rate .99 
Figure 45 - Suggested TDR Thresholds for Determining the Surface Clogging Rate,

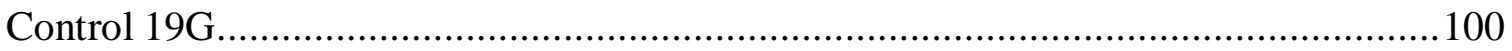

Figure 46 - Runoff Flow during a Rain Event, Control 19G .................................. 102

Figure 47 - Comparison of Clogging Rate on Curbside and Middle, Control 19G ........ 103

Figure 48 - Measured Exfiltration Rates and Recorded Sub-Surface Temperatures for the

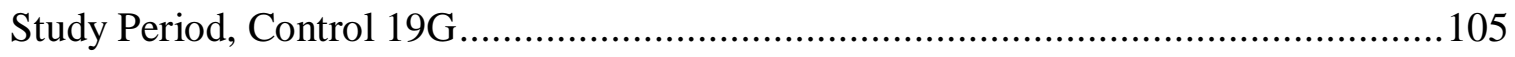

Figure 49 - Measured Exfiltration Rates and Recorded Sub-Surface Temperatures for the

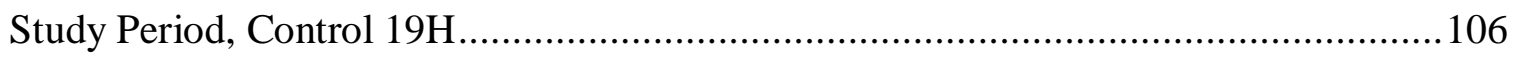

Figure 50 - Average Exfiltration Values for years 2011, 2012, and 2013 for Control 19G

Figure 51 - Average Exfiltration Values for years 2011, 2012, and 2013 for Control 19H

Figure 52 - Temperature Dependency of Exfiltration Rates versus Temperature, Control

$19 \mathrm{G}$ 110

Figure 53 - Temperature Dependency of Exfiltration Rates versus Temperature, Control

$19 \mathrm{H}$ .111

Figure 54 - Fluidity of Water versus Temperature.

Figure 55 - Observed and Corrected for Fluidity of Exfiltration Rates for Year 2013,

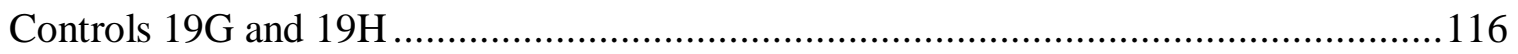

Figure 56 - Estimated Intrinsic Permeability for Control 19G ................................118

Figure 57- Estimated Intrinsic Permeability for Control 19H ................................ 118

Figure 58 - Changes in Average Estimated Intrinsic Permeability Values with Age,

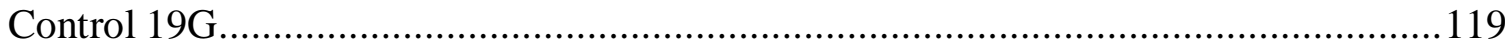


Figure 59 - Changes in Average Estimated Intrinsic Permeability Values with Age, Control 19H...

Figure 60 - Actual Exfiltration Performance from GI Controls 126

Figure 61 - Modeling Exfiltration Performance. 126

Figure 62 - Observed and Modeled Water Levels based on Technique 1, Control 19G. 129 Figure 63 - Observed and Modeled Water Levels based on Technique 1, Control 19H.130 Figure 64 - Observed and Modeled Water Levels based on Technique 2, Control 19G.131 Figure 65 - Observed and Modeled Water Levels based on Technique 2, Control 19H.132 Figure 66 - Suspected Exfiltration Process from Modeling Technique 2, Controls 19G and $19 \mathrm{H}$ 134

Figure 67 - The Model is Reset before Each Water Level Drop and Rise 135

Figure 68 - Changes in Infiltration Capacity for Control 19G, Model by Technique 1 .. 136 Figure 69 - Changes in Exfiltration Performance for Control 19G, Model by Technique 1

Figure 70 - Changes in Infiltration Capacity for Control 19H, Model by Technique 1 .. 137 Figure 71 - Changes in Exfiltration Performance for Control 19H, Model by Technique 1

Figure 72 - Changes in Infiltration Capacity for Control 19G, Model by Technique 2 .. 138 Figure 73 - Changes in Exfiltration Performance for Control 19G, Model by Technique 2 138

Figure 74 - Changes in Infiltration Capacity for Control 19H, Model by Technique 2 .. 139 Figure 75 - Changes in Exfiltration Performance for Control 19H, Model by Technique 2 
Figure 76 - Cumulative Runoff and Captured Volumes, Control 19G 143

Figure 77 - Cumulative Runoff and Captured Volumes, Control 19G .143

Figure 78 - Comparison between Infiltration Performances of Controls 19G and 19H and Role of Intra-event Exfiltration. 144

Figure 79 - Observed Surface Clogging on the Surface of Control 19G, February 2012 148

Figure 80 - Pre (left) and Post (right) Maintenance Photos. Sweeper/Vacuum Truck (top). Pressurized Airjet \#1 (middle), and Vac-Head (bottom). 151 Figure 81 - The Three Methods Used for Restoring Infiltration Capacity of Controls 19G and $19 \mathrm{H}$ 152

Figure 82 - Restoration of Average Surface Infiltration Rates for Different Maintenance Treatments 157

Figure 83 - Restoration of Surface Infiltration Rates for Curbside Locations along Control 19G and Different Maintenane Treatments 158

Figure 84 - Last Curbside TDRs' Measurements after Construction and Airjet \#1, Control 19G 160

Figure 85 - Last Curbside TDRs' Measurements after Construction and Airjet \#3, Control $19 \mathrm{G}$ .160

Figure 86 - Last Curbside TDRs' Measurements after Construction and Vac-Head, Control 19G. 161

Figure 87 - Infiltration Capacity and Surface Maintenance Treatments, Control 19G ... 162

Figure 88 - Infiltration Capacity and Surface Maintenance Treatments, Control 19H ... 162 
Figure 89 - Size Distribution of Collected Samples from Vacuum and Sweeping Maintenance, Control 19G .165

Figure 90 - Size Distribution of Collected Samples from Airjet \#1 Maintenance, Control 19G .165

Figure 91 - Size Distribution of Collected Samples from Airjet \#1 Maintenance, Control $19 \mathrm{H}$ 166

Figure 92 - Size Distribution of Collected Samples from Airjet \#2 Maintenance, Control $19 \mathrm{G}$ .166

Figure 93 - Size Distribution of Collected Samples from Airjet \#2 Maintenance, Control $19 \mathrm{H}$ 167

Figure 94 - Typical Year Rainfall Distribution using Year 2001 Rainfall Data.... 171

Figure 95 - Rain Depth versus Overflow Volume, CSO130

Figure 96 - Residual Plots for the Final Selected Regression Model.

Figure 97 - Modeled and Observed Overflow Volumes versus the Rain Depth, CSO130

Figure 98 - Changes in Modeled Overflow Volume due to Changes of Rainfall Event

Depth.

Figure 99 - Changes in Modeled Overflow Volume due to Changes of 3-Day Antecedent Rainfall Depth 179

Figure 100 - Comparison of Overflow Volumes for Pre and Post Construction of New GI Controls .180

Figure 101 - Comparison of Observed and Modeled Overflow Volumes for Pre and Post Construction of New GI Controls Conditions .181 
Figure 102 - Last Measured TDR Responses Greater than 0.1 Threshold for Airjet Maintenances, Control 19G

Figure 103 - Data Sheet for Estimating the Sediment Attached to the Aggregate..........220

Figure 104 - Data Collection Sheet for Surface Infiltration Tests ….............................223

Figure 105 - Changes in Measured Water Level for Control 19G, 2011 \& 2012...........224

Figure 106 - Changes in Measured Water Level for Control 19G, 2013 ......................225

Figure 107 - Changes in Measured Water Level for Control 19H, 2011 \& 2012...........226

Figure 108 - Changes in Measured Water Level for Control 19H, 2013 ......................227 


\section{LIST OF TABLES}

Table 1 - The MSD's Objectives for CSO Mitigation in CSO130 ...............................48

Table 2 - Characteristics of Control 19G \& 19H ...................................................48

Table 3 - Measured Infiltration Rates along the Length of the Storage Gallery ..............61

Table 4 - Infiltration Rate Values for the Bottom of Trenches of Controls 19G and 19H 61

Table 5 - Measured Attached Solids for \#57 Stone (data source: the EPA)...................63

Table 6 - Measured Attached Solids for \#3 Stone (data source: the EPA) ....................63

Table 7 - Porosity Measurements Results for \#3 Stone...............................................65

Table 8 - Porosity Measurements Results for \#57 Stone...........................................65

Table 9 - Surface Infiltration Testing Locations of Controls 19G \& 19H.....................66

Table 10 - Maintenance Treatment Methods Conducted on Controsl 19G and 19H.......68

Table 11 - Comparison of Rainfall Events for Years 2012, 2013 (Study Period) and 2001

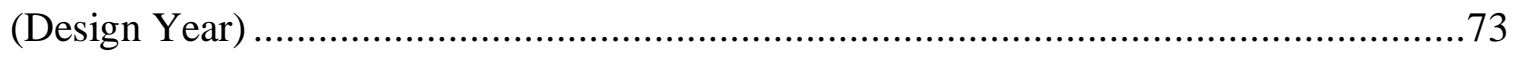

Table 12 - Locations of the Three Pressure Transducers along the Length of each GI

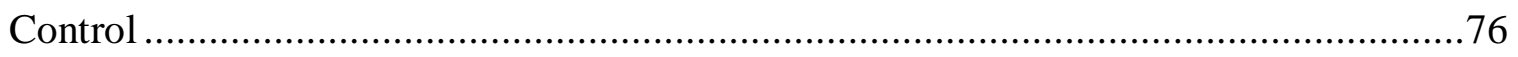

Table 13 - Water Levels and Number of Rainfall Events Analyzed for Estimating the

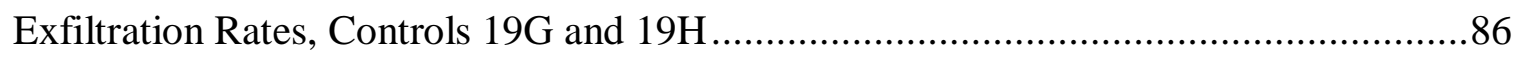

Table 14 - Water Levels and Number of Calculated Exfiltration Rates, Controls 19G and $19 \mathrm{H}$ .104 
Table 15 - Kruskal-Wallis Test Results Comparing the Exfiltration Rate Values in Each

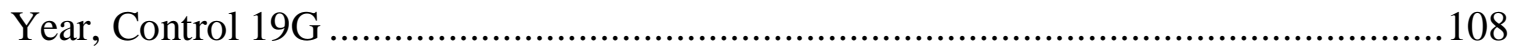

Table 16 - Kruskal-Wallis Test Results Comparing the Exfiltration Rate Values in Each

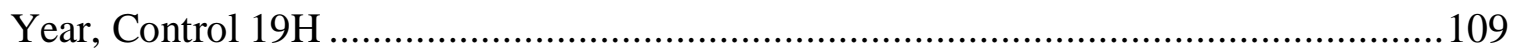

Table 17 - Average Intrinsic Permeability Values Estimated by Considering Fluidity of

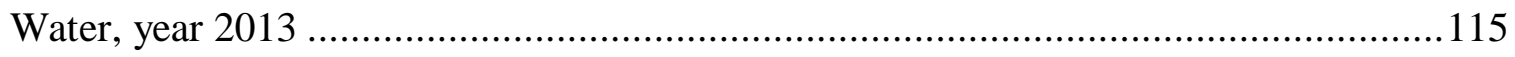

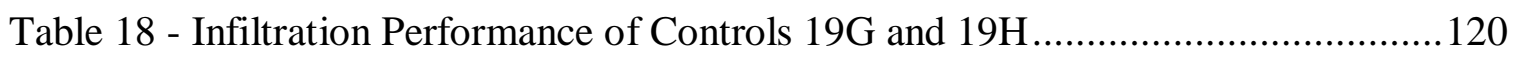

Table 19 - Characteristics of Used Modeling Methods ........................................... 127

Table 20 - R-Squared and Drainage Areas from both Modeling Techniques ................ 133

Table 21 - Initial Surface Infiltarion Test Results, Control 19G.................................... 146

Table 22 - Initial Surface Infiltarion Test Results, Control 19H................................ 146

Table 23 - Average of Baseline Infiltration Rates for Controls 19G and 19H .............. 147

Table 24 - Summary of Maintenance Treatments ............................................... 150

Table 25 - Pre and Post Maintenance Surface Infiltration Test Results, Sweeper Vacuum

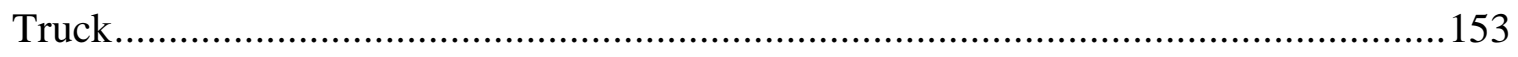

Table 26 - Pre and Post Maintenance Surface Infiltration Test Results, Pressurized Airjet

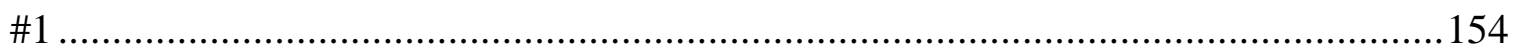

Table 27 - Pre and Post Maintenance Surface Infiltration Test Results, Pressurized Airjet

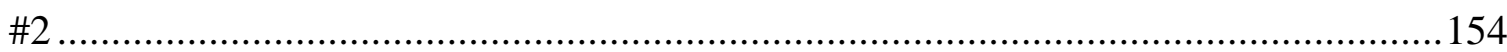

Table 28 - Pre and Post Maintenance Surface Infiltration Test Results, Vac-Head Method .155

Table 29 - Paired Student's T-Tests for Pre and Post Maintenance Surface Infiltration

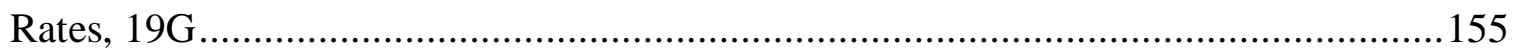


Table 30 - Paired Student's T-Tests for Pre and Post Maintenance Surface Infiltration

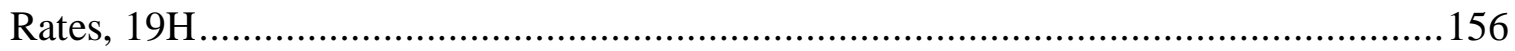

Table 31 - Cumulative Rainfall Depth Effectively Captured after Each Maintenance

Treatment 163

Table 32 - Coefficients of Uniformity and Curvature from Size Distribution Analysis .167

Table 33 - Organic Content Test Results for the First Two Maintenance Treatments.... 168

Table 34 - Size Gradation of Organic Content for Third Maintenance Treatment (Airjet

\#2)

Table 35 - Parameters Selected for Regression Analysis of Overflow Volumes

Table 36 - Predicted and Observed Overflow Volumes for Pre and Post Construction

Conditions

Table 37 - Descrpition of Characters Used in Naming Scheme for Controls 19G \&19H .209

Table 38 - Types of Measurement Made by Each Sensor ........................................210

Table 39 - Reference Table for Two-Character Unique Naming...............................210 


\section{INTRODUCTION}

\subsection{Background}

Federal Water Pollution Control Act, also known as Clean Water Act (CWA) is a federal law passed in 1972 that addresses the concerns regarding the discharges of pollutants and toxic substances to water bodies of the United States. According to the U.S. Environmental Protection Agency (U.S.EPA 2013a) the main objective of the CWA is:

"to restore and maintain the chemical, physical, and biological integrity of the nation's waters by preventing point and nonpoint pollution sources, providing assistance to publicly owned treatment works for the improvement of wastewater treatment, and maintaining the integrity of wetlands."

Under the Clean Water Act, the Environmental Protection Agency (EPA) has defined water pollution programs and water quality standards for pollutants in surface waters. In order to meet the CWA's pollution goals, EPA published the Combined Sewer Overflow (CSO) Control Policy in 1994 (U.S.EPA 2002). The CSO Control Policy offers national guidance to local and federal governments on how to control CSOs and how to meet the pollution control goals defined in Clean Water Act. The policy also requires municipalities with combined sewer systems (CSSs) to develop and implement Long-Term CSO Control Plans (LTCPs) to satisfy the requirements stated by CWA (U.S.EPA 2002; U.S.EPA 2012a). 
The EPA strongly advises communities to consider Green Infrastructure (GI) stormwater controls in mitigating their CSO problems wherever it is feasible and cost effective. Since 2006, the EPA and the Department of Justice have negotiated consent decrees, which incorporate use of GI stormwater controls in CSO management, with cities such as Cincinnati, Indianapolis, Cleveland, Kansas City and Louisville (NRDC 2011).

On December 2008, the Louisville and Jefferson County Metropolitan Sewer District (MSD) entered into a Consent Decree with the US Department of Justice, the U.S.EPA and the Kentucky Department for Environmental Protection (KDEP) to take necessary measures to mitigate the number and volumes of untreated overflows from Louisville's combined sewer system (CSS), and to eliminate sanitary sewer overflows (SSOs) and other unauthorized discharges. These overflow volumes discharge to Ohio River and local streams, in violation of the federal Clean Water Act. The Consent Decree was entered into the Federal Court on April 2009 (MSD 2013a).

MSD prepared a plan, known as the Integrated Overflow Abatement Plan (IOAP), to answer and comply with the requirements of the Consent Decree. The IOAP consists of three inter-dependent volumes: Volume I, Integrated Overflow Abatement Plan (IOAP); Volume II, Final CSO Long-Term Control Plan (LTCP); and Volume III, Final Sanitary Sewage Discharge Plan (MSD 2013a). The Final LTCP includes a mixture of Green Infrastructure (GI) and Gray solutions to control the wet weather CSOs. The GI solutions include using Stormwater Control Measures (SCMs) such as permeable pavements, green roofs, rain gardens, etc., while the gray solutions include options such as storage, treatment, conveyance/transport, and sewer separation. Approximately 17 percent of the long-term plan budget to control CSOs is allocated for GI solutions (MSD 2013b). MSD is committed 
to develop and implement a monitoring plan to evaluate the performance of various green infrastructure elements (MSD 2009). The knowledge gained from this monitoring effort will be used as a decision making factor in the future implementation of GI controls by MSD (MSD 2009).

One of MSD's initial monitoring efforts to CSO abatement, includes implementing a set of GI stormwater controls in CSO130 sewershed in lieu of a planned gray retention basin. CSO130, located in a small urbanized neighborhood at the East of Louisville, is 11 hectares (28 acres) and the overflow volumes from this watershed discharges to Beargrass Creek which flows into the Ohio River.

The MSD and the URS Corporation (URS) were responsible for design and implementation of the GI controls throughout the CSO130 basin and the EPA's office of Research and Development (EPA) and the Center for Infrastructure Research (CIR) at the University of Louisville were tasked with preparing a monitoring plan to evaluate the individual performances and overall effectiveness of GI controls in mitigating the CSO problem in the CSO130 sewershed. The first phase of the project was completed in December 2011 by implementing two permeable pavement systems along the parking lanes of Adams Street. Both GI controls were embedded with time domain reflectometers (TDRs), thermistors, and piezometers (pressure transducers). Monitoring data has been recorded from all these sensors at 1 -minute intervals since December $13^{\text {th }}, 2011$.

The collected monitoring data has been used to evaluate the hydrological performance of both permeable pavement systems in CSO130. Hydrological performance is defined as a GI control's ability to capture the runoff volume (infiltration capacity) and 
then pass the captured volume into the surrounding and underlying soil layers (exfiltration performance).

\subsection{Statement of the Problem}

On December 2011, two permeable pavement systems were installed along the parking lanes of Adams Street, located in the Butchertown neighborhood, in Louisville, Kentucky. Implementation of these GI systems happened as part of MSD's Integrated Overflow Abatement Plan, in which MSD decided to manage stormwater entering the CSS by using GI stormwater controls where the construction costs of these systems are no larger than the alternative gray solution for the given sewershed basin.

Both pavement systems utilized rather unconventional designs. This was done for a number of reasons, among which are:

- The MSD wanted to install these GI controls in public property to maintain control of any future maintenance treatments required to ensure the proper performance of GI controls.

- Soil layers suitable for proper exfiltration of captured stormwater were located at depths of 10 to 15 feet from the street surface.

- Drainage areas for both GI controls were larger than those typical for permeable pavements systems.

For these reasons the permeable pavement systems were constructed along the parking lanes of Adams Street. Each system was 8 feet wide and ranged in length from 55$\mathrm{ft}$ to $120-\mathrm{ft}$. Both pavement systems cover a 2 -ft deep storage gallery with a $10-\mathrm{ft}$ deep trench excavated along the bottom of the storage gallery. The deep trench provides access 
for the captured runoff volume to deeper soil layers that have higher hydraulic conductivity values. For both pavement systems, the ratio of the drainage area to the permeable pavement surface is more than three times larger than it is recommended for permeable pavements by different design manuals. The reason was that the MSD intended to fully test and exercise the application of these GI controls to achieve desirable CSO mitigation for the given sewershed. This required maximized runoff volume captured and therefore large drainage ratios. A comprehensive monitoring effort was conducted to evaluate the effectiveness of the GI controls under this circumstances.

A few weeks after the construction of permeable pavement systems, clogging of the surface and at the interface of subsurface storage layers and the underlying soil layer was observed and this predominated changes in hydrological performance of GI controls. While the surface clogging was observed to advance quickly from the up-gradient edge to down-gradient edge, decrease in sub-surface exfiltration rates of captured runoff volume in both GI controls was also evident. The surface clogging happens because the stormwater runoff carries sediments and fine materials that get trapped between the gaps of the pavers as the runoff volume infiltrates into the permeable pavement system. The subsurface clogging is also the direct result of the sediment accumulation at the bottom of the GI stormwater control.

Developing an assessment tool for effective monitoring of changes in hydrological performance of GI controls is essential. Such a tool can also be utilized for evaluating the effectiveness of surface maintenance treatments, as well as for identifying and defining the roles of contributing factors that can potentially affect the infiltration performance and exfiltration capacity of permeable pavement systems. Such factors can include: age of the 
GI stormwater control, cumulative surface runoff captured to date, sub-surface temperature, and antecedent moisture conditions of sub-surface soil layers.

Limited studies have been carried out on the hydrological performance of permeable pavement systems. Also, the majority of these studies are conducted in small and controlled environments, such as a small parking lot, where mostly the effluents from GI controls are recollected through a piping system, rather than allowing it to naturally exfiltrate to surrounding and underlying soil layers. There are even less studies of the seasonal changes as a contributing factor to hydrological performance of a GI stormwater control.

During construction of both permeable pavement systems, both GI controls were embedded with a number of sensors, including piezometers (pressure transducers), time domain reflectometers (TDRs) and thermistors. The instrumentation of both controls enabled investigation of the hydrological performance of permeable pavement systems and the roles of contributing factors to each GI control's individual performance.

\subsection{Objectives of this Research}

As previously discussed, there are few studies of the performance and effectiveness of GI stormwater controls, and these few studies have been either too small (physical scale) or too short (in duration), so there is not enough data to establish the confidence for longterm decisions about using GI stormwater controls.

The current study focused on monitoring the changes in the hydrological performance of the two permeable pavement systems and its contributing factors. The longterm monitoring effort will enable us to answer the two following main questions: 
- How will the hydrological performances of individual GI stormwater controls in CSO130 change with time?

- How will the hydrological performances of individual GI stormwater controls in CSO130 change with seasonal changes?

The data from embedded pressure transducers shows that as it rains and the water infiltrates into the controls, the measured water level rises quickly and then it falls gradually as the water exfiltrates into the underlying and surrounding soil layers. A spreadsheet model is developed by utilizing each control's drainage area, hydraulic conductivity values for surrounding soil layers, and dimensions of each GI control. The model calculates the inflow and outflow volumes and predicts the water level inside the control for each rain event. The model's predicted water levels are compared to the recorded water levels by the embedded pressure transducers. The developed model predicts the GI control's initial performance and when compared to recorded data, quantifies the progression of clogging at the surface and at the interface of storage layer and underlying soil layer.

The developed model is also used to assess and track long-term and seasonal changes in both surface infiltration capacity and sub-surface exfiltration performance of permeable pavements during the first two year of their service lives. This was done by separating the rise and drop of the observed water level into independent events. The model was reset before each event and the results at the end of events are used to analyze hydrological performance. The rise and drop of water level are used for assessing the infiltration and exfiltration process, respectively. 
Calculating the total surface runoff volume captured by each control was complex. The rise in water level couldn't be used alone since some of the captured volume was exfiltrating as more stormwater was infiltrating into the storage gallery during the rainfall event. This phenomena is referred to as intra-event exfiltration. Once the model was set up and calibrated, it was used to calculate the captured runoff volume by considering the intraevent exfiltration process.

While the manual surface infiltration tests and TDR data confirm the progression of the clogging on the surface, it wasn't clear what "clogged" means in terms of "volume of captured water". Similarly, the initial monitoring results confirmed significant decreases in exfiltration rates of captured runoff volume. While the decrease in exfiltration rates were evident, it was not known how this could affect the overall performance of the GI controls. The modeling results provide further insight into the actual performance of GI controls during surface infiltration and sub-surface exfiltration processes. The two year continuous monitoring data provided an opportunity to study the seasonal changes in hydrological performance of both GI stormwater controls.

One of the key objectives of this study is to evaluate and compare different surface maintenance treatment methods or permeable pavement systems. Since the installation of GI stormwater controls in December 2011, three different methods have been used to restore the infiltration performance of permeable pavements. The effectiveness of these methods were studied using the results obtained from manual surface infiltration tests, TDR data, and developed model for GI controls. 
The last part of this research is focused on evaluating the overall effectiveness of installed GI controls in mitigating the CSO issue. The overflows from pre and postconstruction conditions are compared and a regression analysis is used to identify significant factors affecting overflow volumes and to model the pre-construction conditions. Using the model, the post-construction overflow volumes are compared to preconstruction conditions.

This research is expected to give designers a better understanding of long-term hydrological performance of infiltration GI stormwater controls, and factors that can affect system performance. It can also serve as a guide for future researchers in developing costeffective techniques for monitoring GI stormwater controls. 


\section{LITERATURE REVIEW}

\subsection{Introduction}

The Louisville MSD is interested in mitigating the number and volume of overflows from the city's combined sewer system (CSS) and has incorporated the use of Green Infrastructure (GI) Stormwater Control Measures in the current CSO Long-Term Control Plan. One of the first demonstration projects, in which the MSD is planning to solve the CSO problem by implementation of GI stormwater controls, includes installation of a number of permeable pavement systems in a small sewershed. The MSD is also interested in monitoring the long-term performance and effectiveness of these systems so the information can be used as a decision making factor in the future.

The hydrological performance of permeable pavements is easily affected by surface clogging which is caused by the sediments that are washed onto the control and subsequently trapped between the pavers' gaps. Clogging at the bottom of the storage gallery, at the interface of storage layer and underlying soil, causes reductions in exfiltration rates of the captured runoff volume and consequently a decrease in exfiltration performance of the GI stormwater control. While long-term performance of permeable pavement systems is affected by clogging, temperature is another contributing factor. Changes in local temperature values, affect the viscosity of water and subsequently flow of water through the interstices of the porous media. 
First few sections of this chapter are dedicated to definitions and related issues of Combined Sewer Systems, Effects of Urbanization on the Hydrological Cycle and Combined Sewer Overflows, and GI Stormwater Control Measures. The next sections of this document include a brief literature review on previous studies conducted on hydrological performance of permeable pavement systems and the parameters and factors that affect the performance of this type of GI stormwater controls.

\subsection{Combined Sewer Systems}

A combined sewer system (CSS) is a type of wastewater collection system that conveys domestic, commercial, industrial waste water, and stormwater runoff all through single pipe systems to a publicly owned treatment works (POTW) (U.S.EPA 1999a; U.S.EPA 2004). During normal dry weather conditions, untreated wastewater collected in the combined sewer system is routed through a wastewater treatment plant before it enters natural waterways. During heavy rainfall conditions, the sewage flow may exceed the capacity of the sewer system, and as a result, a mixture of sewage and stormwater is discharged at a combined sewer overflow (CSO) point prior to the treatment plant. The discharge continues until the flow drops below capacity of sewer network. Most CSSs are designed to discharge the excess flow to surface water bodies such as streams and rivers. Release of this excess flow is necessary to prevent flooding in homes, basements, businesses, and streets. Overflow volumes and frequencies vary from one system to another and from outfall to outfall (U.S.EPA 2004). Figure 1 compares combined and separate sewer systems and their perfomances during dry and wet weather conditions. 


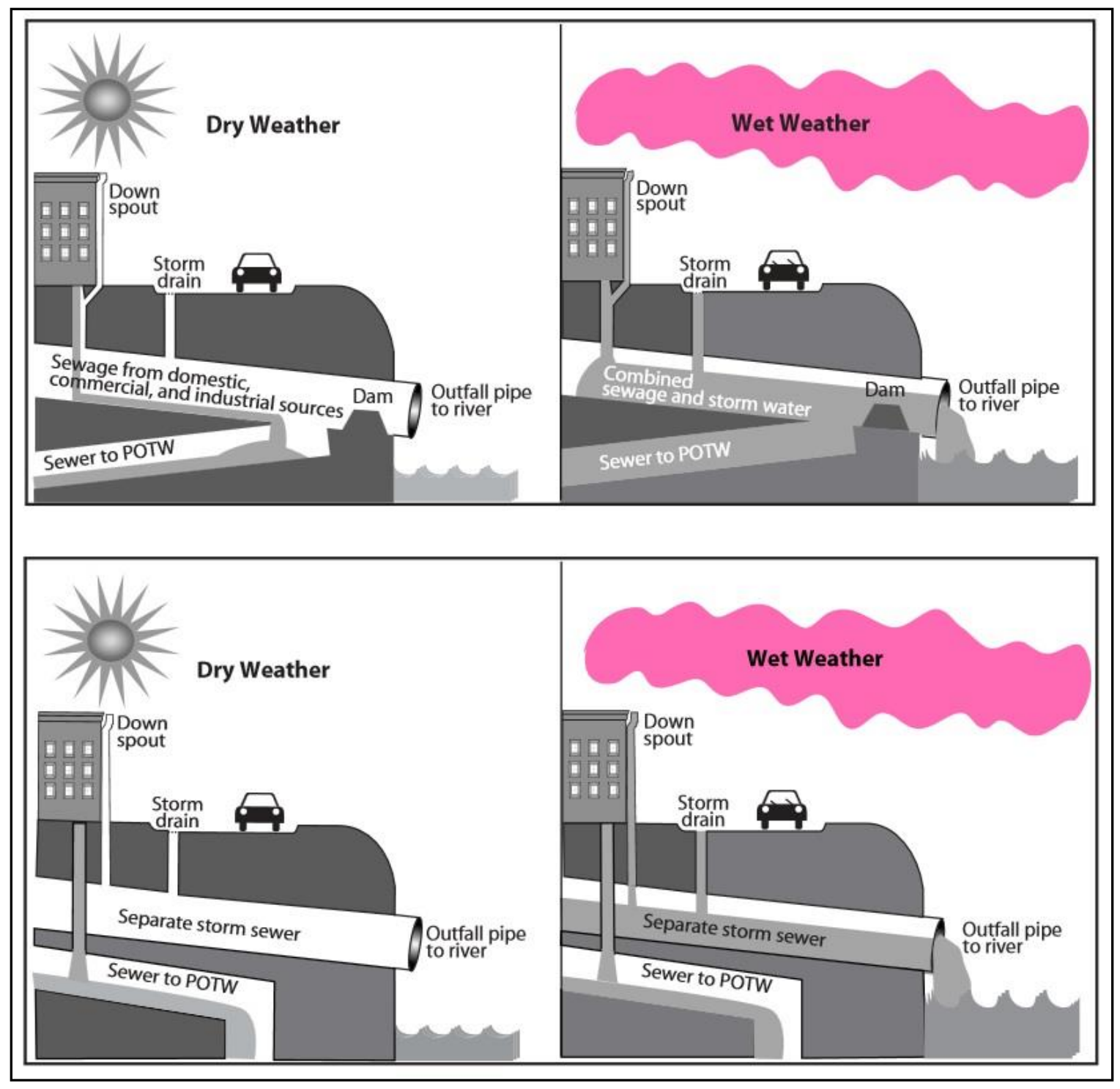

Figure 1 - Combined (top) and Separate (down) Sewer Systems (U.S.EPA 2004)

Most historic cities in Europe (e.g., Paris, London or Rome), as well as North America, have combined sewer systems (Weyrauch et al. 2010). Within the U.S., the majority of communities with combined sewer systems are located in the Northeast and Great Lakes regions, while relatively few are located in the Midwest, Southeast, and Pacific Northwest (U.S.EPA 2004; U.S.EPA 2012b). Figure 2 shows the approximate location of 
these communities in the U.S. Louisville's sewer system, which was first constructed in 1800s, is a CSS type.

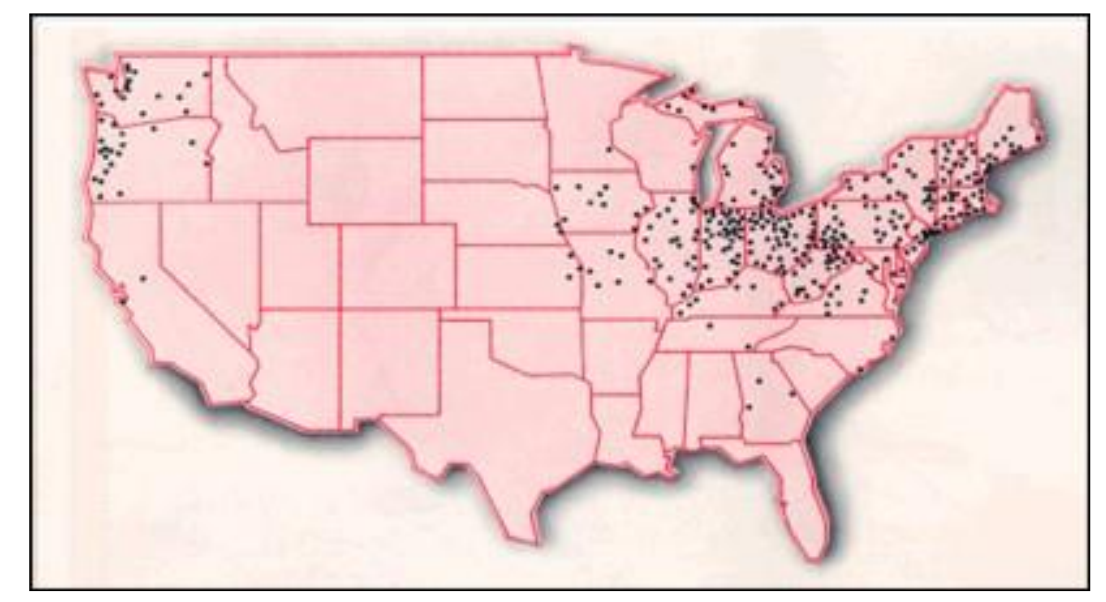

Figure 2 - Communities with Combined Sewer Systems (U.S.EPA 2012b)

The impact of CSOs on quality of water resources is a major concern for the approximately 772 communities with about 40 million residents in the United States (U.S.EPA 2012b). Moffa (1997) refers to CSOs as "the single biggest obstacle to achieving the swimmability goals of the 1972 Clean Water Act Amendment". CSO discharges include a mix of human, commercial, and industrial wastes as well as pollutants washed by the surface runoff from parking lots and streets, and they may contain high levels of suspended solids, biochemical oxygen demand (BOD), oil and grease, floatables, toxic pollutants, pathogenic microorganisms and other sorts of contaminants (U.S.EPA 1999a; U.S.EPA 2004). Many CSOs discharge to surface waters located at heavily populated urban areas cause aesthetic impairments, or beach closures, and may even have adverse impacts on human health and aquatic life. Some of the water-borne diseases include hepatitis, or skin and ear infections (Field et al. 2003). 
A study by Weyrauch et al. (2010) examined the contribution of combined sewer overflows (CSO) to loads and concentrations of trace contaminants in receiving surface water. The results of the study indicate that CSO may be a significant source of sewagebased trace substances and can lead to potentially problematic acute concentrations in receiving waters during storm events (Weyrauch et al. 2010). Samrani et al. (2004) investigated the existence of various particle types that can be found in sewage and CSOs. Through detailed analysis of individual particles found in CSOs, they conclude that various sources such as urban surfaces, soil surfaces, or sewer sediments, contribute to CSOs (El Samrani et al. 2004). Studies have also shown that there is a relationship between CSOs, wastewater discharges and delayed benthic oxygen demand. Oxygen is a good indicator of the health of an aquatic environment and therefore CSOs, by causing noticeable reductions of dissolved oxygen in water, will have an adverse effect on phytoplanktonic activity and food web of the aquatic habitat (Even et al. 2007; Even et al. 2004).

\subsection{Effects of Urbanization on the Hydrological Cycle}

The hydrological cycle, also known as the water cycle, describes the constant movement of water between land, water bodies, and the atmosphere. The schematic diagram in Figure 3 shows the water cycle between oceans and land. In this graph the pools (shown in blue) are in cubic miles and fluxes (shown in black) are in cubic miles per year. 


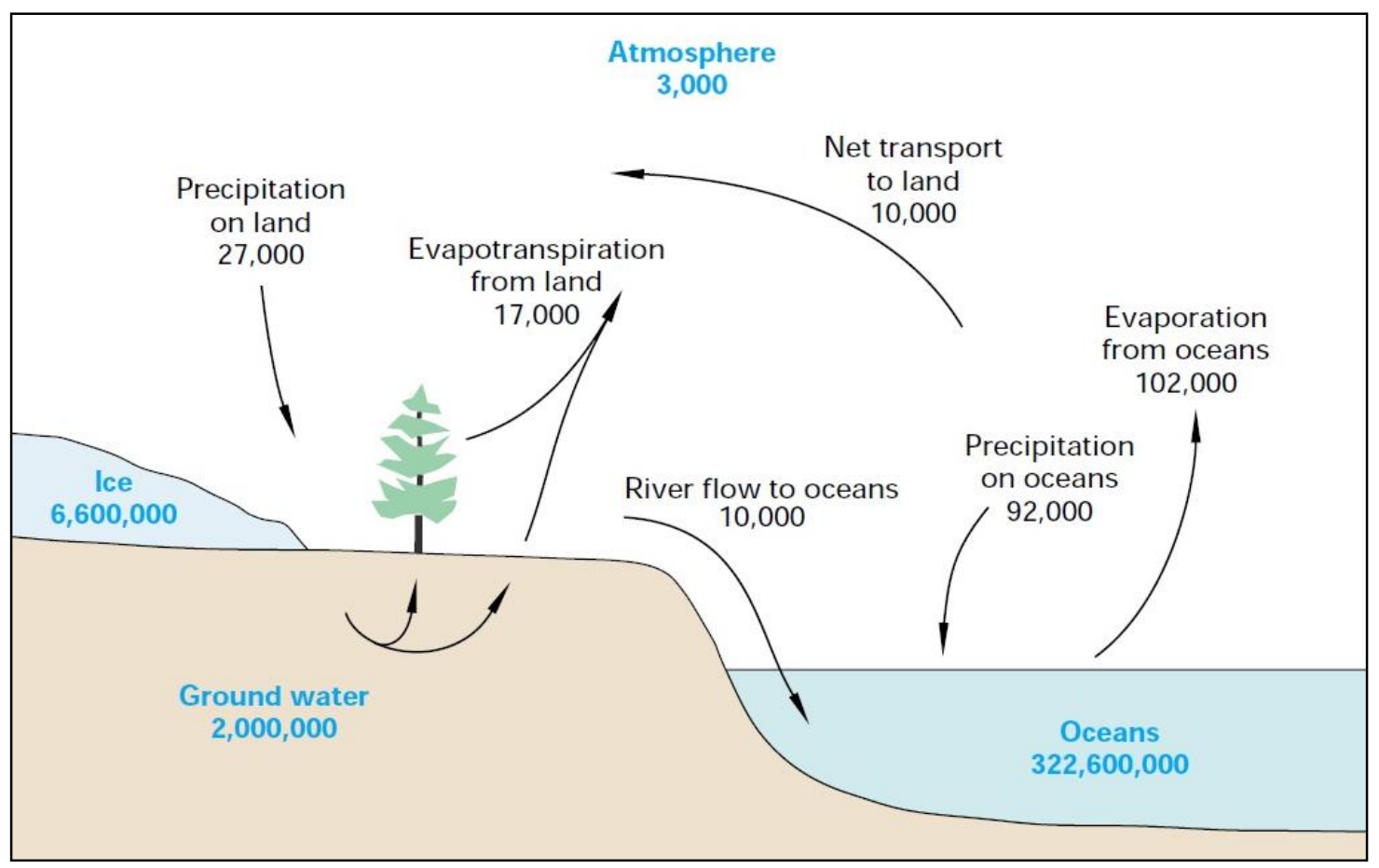

Figure 3 - Water Cycle between Land, Ocean, and Atmosphere (Winter et al. 1998).

As it can be seen on Figure 3, approximately $97 \%$ of the global water is stored in the ocean and just a fraction of it is usable fresh water. As the rain hits the ground, a portion of it evaporates, some infiltrates into the ground, and the rest flows downhill overland, eventually reaching the ocean and lakes through streams and rivers. Impervious surfaces, which are one of the major results of urbanization, alter the hydrologic cycle and the way water is stored and transported by preventing the infiltration of water into the soil (Arnold and Gibbons 1996). Such impervious surfaces include roads, parking lots, and rooftops. As little as 10 percent impervious cover in a watershed can result in stream degradation (U.S.EPA 2003). In densely urbanized areas, over half of the total rainfall might turn into surface runoff (Figure 4). 


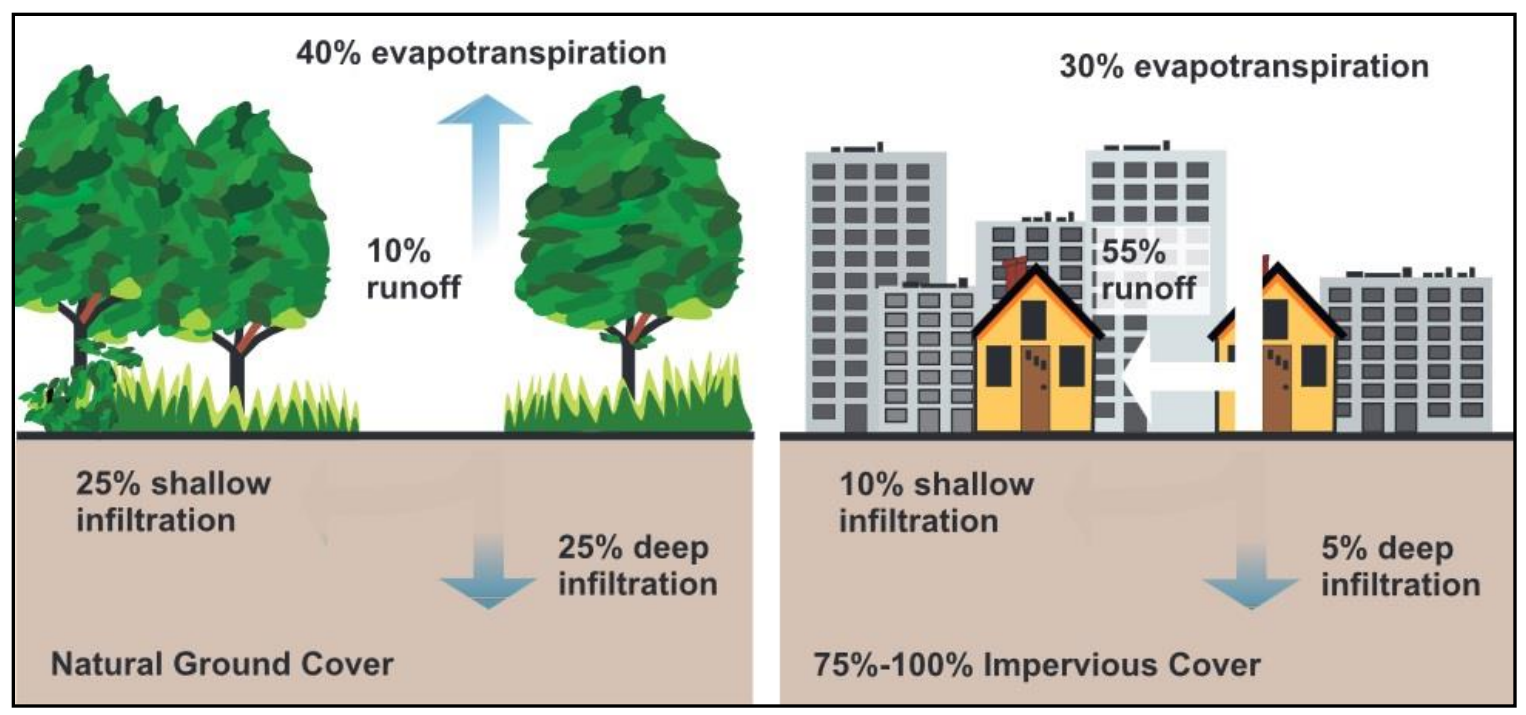

Figure 4 - Relationship between Impervious Cover and Surface Runoff (U.S.EPA 2003)

The high percentage of impervious surfaces, results in shorter lag times between onset of rainfall, and subsequently higher runoff peaks, and total volume of runoff (Shuster et al. 2005). Increase in the ratio of impervious surfaces to pervious surfaces, creates an increase, not only in the volume of surface water but also the rate of flow that is delivered to surface water bodies (Galster 2006).

Imperviousness also has an adverse effect on streams and rivers. The higher peak flow velocities, which are the results of imperviousness, can degrade stream channel, erode the stream banks, and degrade the water quality (U.S.EPA 2012d). Figure 5 shows the general relationship between impervious coverage and stream health of a watershed. As shown on the graph, stream health degradation first occurs at $10 \%$ of impervious coverage and at $30 \%$ the degradation becomes so severe that it almost becomes unavoidable (Arnold and Gibbons 1996). 
In summary, impacts of impervious surfaces can be divided into four different categories (CWP 2003):

- Hydrological Impacts: include impacts such as increased runoff volume, increased discharge peak rate, decreased baseflow, etc.

- Physical Impacts: include impact such as channel enlargement, bank erosion, etc.

- Water Quality Impacts: include increased concentrations of nutrients, trace metals, hydrocarbons, etc.

- Biological Impacts: include adverse effects on aquatic community such as: fish diversity, aquatic insects' diversity, etc.

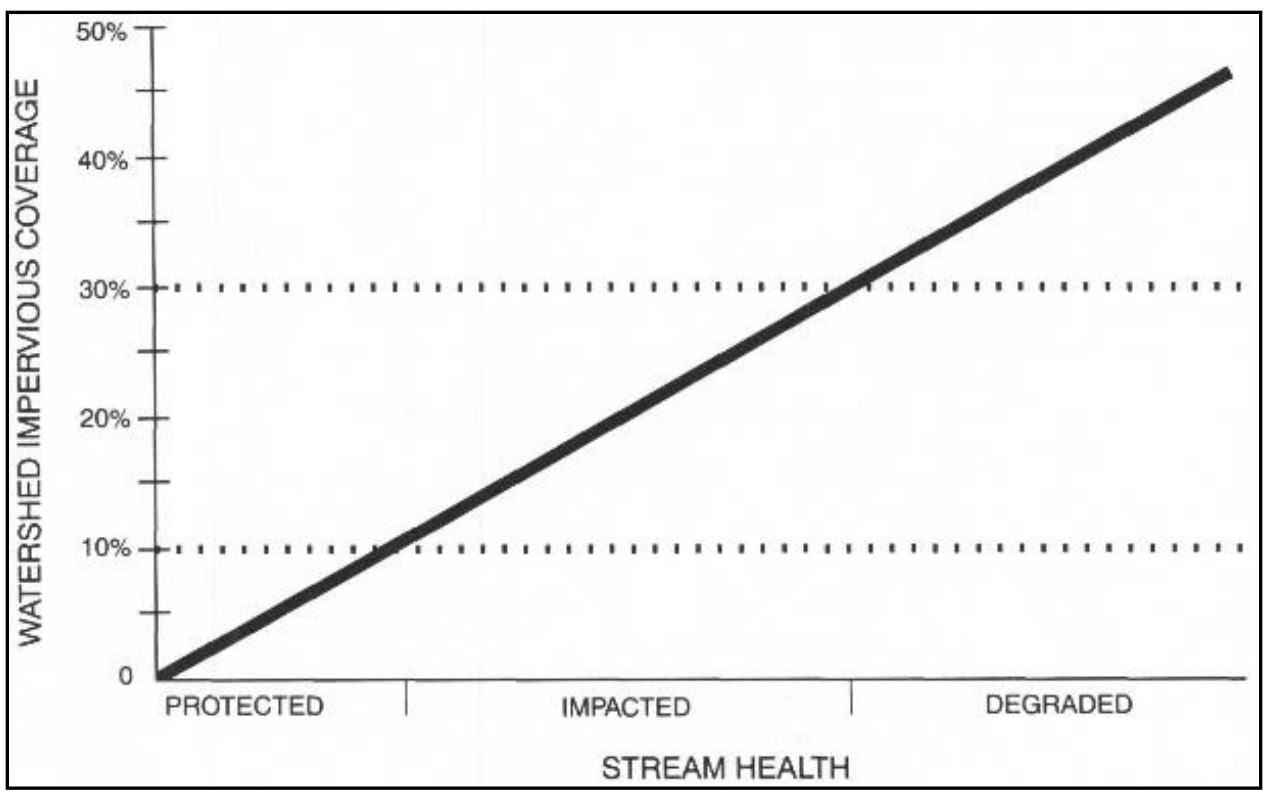

Figure 5 - Relationship between Imperviousness Coverage and Stream Health (Arnold and Gibbons 1996; U.S.EPA 2012d) 
In cities with combined sewer systems, an increase in percentage of impervious areas will result in an increase in runoff volume and runoff coefficient, which will cause higher frequencies and durations of combined sewer overflows (CSOs). In a study by Pyke et al., (2011) the relative influence of changes in impervious cover, precipitation volume, and event intensity on stormwater runoff were investigated by using a stormwater model. The results indicate that change in annual stormwater runoff volume is most sensitive to changes in percentage of impervious cover (Pyke et al. 2011). Booth (2002) reports that once the effective impervious area in a watershed exceeds 10 percent, a post-development 2-year storm event produces a discharge equal or greater than a pre-development 10-year storm event (Booth et al. 2002).

Runoff coefficient is a measure of the site response to rainfall events and is defined as the ratio of runoff depth to rainfall depth. Figure 6 shows the relationship between the percentage of watershed imperviousness and runoff coefficient. This graph is developed after monitoring runoff for over 40 sites across the United States (Schueler 1994). 


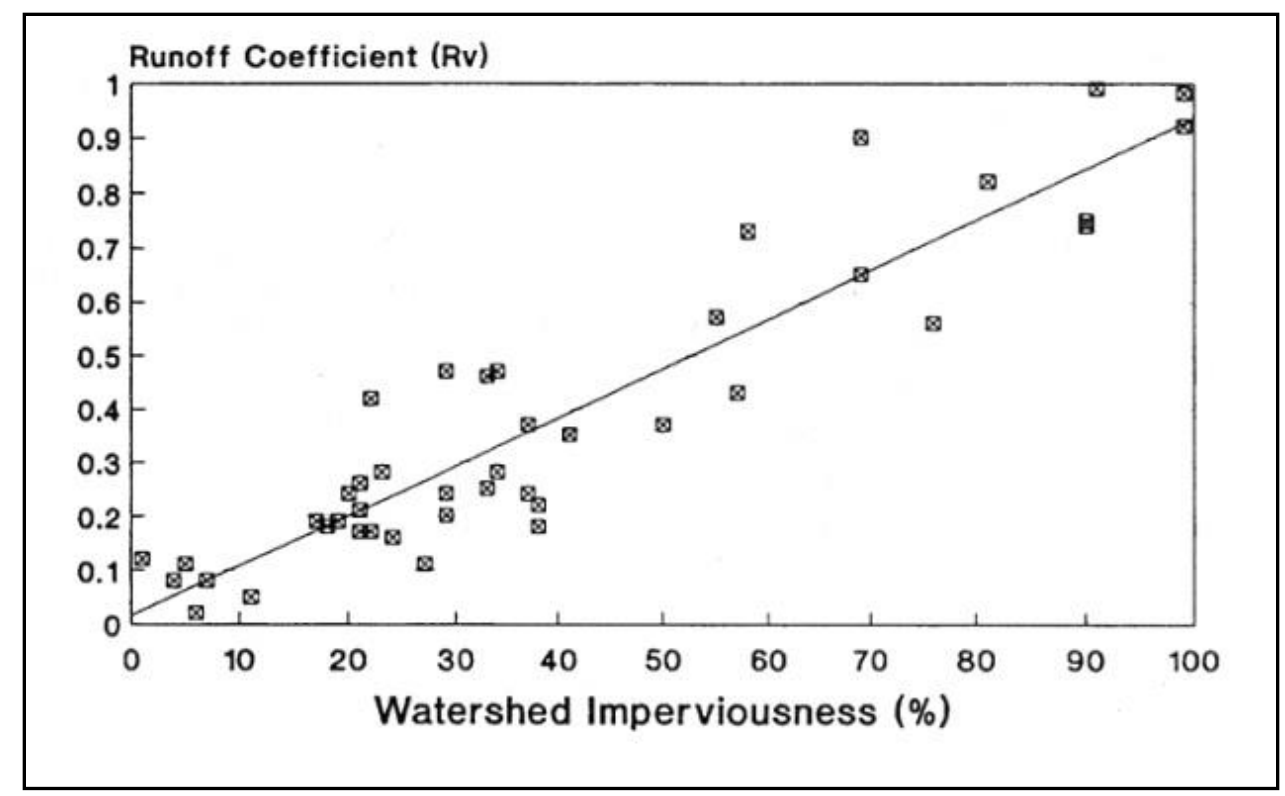

Figure 6 - Relationship between Watershed Imperviousness and Runoff Coefficient (Schueler 1994).

\subsection{Green Infrastructure Stormwater Control Measures}

Wherever natural ground covers, such as grasslands and forests, are replaced by rooftops and roads, the movement of water across the landscape is radically altered (Booth and Leavitt 1999). In such areas the runoff water cannot soak into the ground and flows on the surface. Adverse effects such as flooding, channel erosion, land sliding, and destruction of aquatic habitat are some of the results of this phenomenon. This stormwater surface runoff carries pollutants from the urban landscape and can degrade the quality of the receiving waters. Higher flows can cause erosion and flooding in urban streams, damaging habitat, property, and infrastructure. Nearly all these water quantity problems result from one underlying cause: loss of the water-retaining function of the soil in the urban landscape (Booth and Leavitt 1999). 
In order to control stormwater runoff, engineers and hydrologists have used various techniques to reduce the volume or delay the arrival of water that reaches the sewer and/or river system. Among such techniques are Green Infrastructure (GI) Stormwater Control Measures. GI Stormwater Controls are systems or practices that use or mimic natural processes to infiltrate, evapotranspirate (refers to combined effects of evaporation and transpiration), or reuse stormwater or runoff on the site where it is generated (U.S.EPA 2013b). Such practices are used to mitigate the quantity of stormwater surface runoff in urban areas. GI controls also have a substantial impact on mitigating nonpoint source pollution caused by stormwater runoff. Green Infrastructure principles are based on controlling runoff volume at the source, unlike conventional stormwater solutions that are designed to collect, convey and mitigate the stormwater at a downstream location (U.S.EPA 2000).

Unlike single-purpose gray stormwater infrastructure, which use pipes to dispose of rainwater, green stormwater infrastructure uses vegetation and soil to manage rainwater at source. GI stormwater controls provide not only stormwater management, but also flood mitigation, air quality management, and much more. While most of GI stormwater controls are literally green, since they consist of trees, plants, and vegetation, other GI controls, such as permeable pavements, are also categorized as green because they can provide the water infiltration and retention capabilities of natural systems (NRDC 2011).

In the literature, GI stormwater controls are also referred to as Stormwater Best Management Practices (BMPs), Low Impact Developments (LIDs) or Stormwater Control Measures (SCMs). In United Kingdom they are referred to as Sustainable Urban Drainage 
Systems (SUDS) and Stormwater Quality Improvement Devices (SQID) in Australia (NCHRP 2006).

Advantages of GI stormwater controls can be divided into four different categories:

- Stormwater Volume Control

- Pollutant Removal

- Water Conservation

- Non-Water Benefits

\subsubsection{Stormwater Volume Control}

Green infrastructure practices' approach toward stormwater management is in contrast with conventional stormwater management techniques, which are focused on controlling extreme rainfall events. During design of conventional systems, more frequent and smaller rainfall events are usually ignored while such events could produce $85 \%-95 \%$ of total annual runoff volume in the watershed (NRDC 2011). GI stormwater controls are designed to capture most of the runoff volume associated with smaller and more frequent rainfall events, resulting in significant reduction of discharge volumes and pollutant loads to water bodies. Green Infrastructures benefit combined sewer systems by decreasing the total volume of surface runoff that enters the sewer system, thus reducing the number and size of overflows.

\subsubsection{Pollutant Removal}

GI stormwater controls are effective in reducing pollutants loads, not only by reducing runoff volumes, but also by removing them directly from stormwater through filtering. This could be another advantage of GI stormwater controls, especially for 
separate sewer systems since they don't conventionally provide additional treatment before discharging the collected stormwater (NRDC 2011). Some of the GI stormwater controls reduce the pollutant transport capacity and overall pollutant loading by increasing the time of concentration $\left(\mathrm{T}_{\mathrm{c}}\right)$ and velocity of runoff flow (U.S.EPA 2000). The time of concentration $(\mathrm{Tc})$ is the amount of time it takes for surface runoff to travel from most distant point in a watershed to the watershed outlet.

\subsubsection{Water Conservation}

Some of the Green Infrastructure Controls, such as rainwater harvesting techniques (rain barrels and cisterns), can be used to conserve water by capturing runoff water, typically from rooftops, for future reuse (NRDC 2011). The collected rainwater can be

temporally stored for later use, such as irrigation of gardens or lawns. Rain harvesting techniques can play a substantial role on runoff volume reductions from home rooftops (Jones and Hunt 2010). Such techniques have low to medium installation costs and require little or no maintenance cost from the system's owner (SEMCOG 2008; U.S.EPA 2013c).

\subsubsection{Non-Water Benefits}

Unlike conventional stormwater management systems, GI stormwater controls can have other benefits in addition to reducing stormwater runoff volume and pollution (NRDC 2011). Among such benefits are:

- Improved air quality: Since most of the GI stormwater controls include some sort of vegetation, they can have a positive impact on air quality. Plants filter air and capture pollutions such as carbon monoxide and ozone (NRDC 2011; U.S.EPA 2013b). Total annual air pollution removal by 
urban trees in the United States is estimated at 711,000 metric tons $(\$ 3.8$ billion value) (Nowak et al. 2006).

- Lower air temperature: GI stormwater controls that consists of plants, cool the air through evapotranspiration. Evapotranspiration is the return of moisture to air from soils and plants and refers to combined effects of evaporation and transpiration (NRDC 2011).

- Reduced urban heat island effect: Urban heat island is a metropolitan area that has a temperature significantly higher than rural or suburban areas. This phenomenon happens mostly because of the large ratio of imperviousness of the urban areas. Impervious areas usually have darker surfaces and tend to absorb more heat than natural covers. Some GI practices, such as green roofs, can decrease the heat island effect by reflecting the sunlight and absorbing less heat (NRDC 2011). Also, under wet conditions, permeable pavement systems could give lower surface temperatures than impermeable surfaces (Li et al. 2013).

- Reduced energy use: Green Infrastructure can reduce energy consumption by reducing local temperatures and providing additional insulation for rooftops (green roofs) (NRDC 2011; U.S.EPA 2013b).

\subsection{Different Types of GI Stormwater Controls}

Various design manuals have used different terms and classifications to define different types of GI stormwater controls. In this research we used the terminology as used by the Environmental Protection Agency (EPA). The EPA lists the following as different types of GI Stormwater controls that can be used throughout a watershed (U.S.EPA 2013b): 
- Rain Gardens also known as bioretention cells, are shallow vegetated surfaces with a porous backfill that collect and absorb runoff from rooftops, parking lots and streets. The vegetated surface is sometimes underlain by a layer of sand or gravel that acts as a storage and infiltration bed (MSD 2011; U.S.EPA 2013b).

- Bioswales are vegetated or mulched stormwater conveyance systems that provide treatment and retention for the captured runoff. Bisowales are usually in form of a broad, shallow, and gently sloped channel with deep rooted vegetation that help in filtering the runoff water. The bioswales promote infiltration and reduce the flow velocity of stormwater runoff (MSD 2011; U.S.EPA 1999b; U.S.EPA 2013b).

- Downspout Disconnection refers to rerouting downspouts that convey rooftop runoffs to drain the collected stormwater to other types of GI stormwater controls such as rain barrels, cisterns, or rain gardens. This GI control practice could have a great benefit for communities with combined sewer systems by stopping the stormwater from reaching sewer systems (MSD 2011; U.S.EPA 2013b).

- Rainwater harvesting are systems used for collecting and storing stormwater for future reuse. These systems are often used with downspout disconnection to capture the rooftop runoff in a rain barrel or cistern. The stored water can be used for landscape watering, or flushing toilets (MSD 2011; U.S.EPA 2013b). 
- Planter Boxes are very similar to rain garden in their design purpose and they provide stormwater management benefits to detain, filter and infiltrate the captured runoff. They might have open bottoms to allow slow infiltration of collected stormwater to the underlying soil layers. Planter boxes are especially suitable for space-limited dense urban areas (MSD 2011; U.S.EPA 2013b).

- Green Roofs are building roofs that are planted over a waterproof membrane with growing media and vegetation. The vegetation provides rainfall infiltration and evapotranspiration of collected stormwater. Green roofs are more suitable for urban areas and can be used to reduce stormwater runoff from commercial, industrial, and residential buildings. Green roofs' benefits are not limited to stormwater management as they can be helpful in reducing the rooftop temperatures as well (MSD 2011; U.S.EPA 2013b).

- Urban Tree Canopy also known as urban forestry, are used in urban areas to restore some of the benefits provided by trees. Trees help to reduce and slow stormwater by intercepting precipitation in their leaves and branches. Non-stormwater management benefits of tree canopies include: reduction of the heat island effect, reduction of soil erosion, soil stabilization, and reducing the air pollution (MSD 2011; U.S.EPA 2013b).

- Permeable Pavements are pavement surfaces that infiltrate, treat, and sometimes store stormwater runoff. Permeable pavements are constructed from several materials such as pervious concrete, porous asphalt, and, permeable concrete pavers. Permeable pavements are used to infiltrate the 
precipitation that falls directly on it or runoff from adjacent impervious surfaces (Fassman and Blackbourn 2011). In addition to reducing surface stormwater runoff, permeable pavements can trap suspended solids and filters pollutants from the runoff stormwater (MSD 2011; U.S.EPA 2013b). In the next section permeable pavement systems are discussed in more detail.

\subsection{Permeable Pavement Systems}

The use of permeable pavement reflects an effort to alter the seemingly inescapable relationship between new urban development and increased impervious surface area (Arnold and Gibbons 1996; Schueler 1994). Permeable pavement systems are alternatives to traditional asphalt or concrete surfaces that allows the surface runoff to drain through the permeable surface to a stone reservoir underneath. The reservoir temporarily stores stormwater before infiltrating it into the underlying and surrounding soil layers (U.S.EPA 2012c). The appearance of the alternative surface is often similar to asphalt or concrete, but it is manufactured without fine materials and instead incorporates void spaces that allow for storage and infiltration. The differences in runoff responses from permeable and impermeable surfaces are quite dramatic. At least where soil conditions are suitable, permeable pavements are quite successful at managing surface runoff from small and moderate storms (Booth and Leavitt 1999; Collins et al. 2008).

Permeable pavement systems are suitable for a wide variety of residential, commercial and industrial applications, yet are confined to light duty and infrequent usage, even though the capabilities of these systems allow for a much wider range of usage (Scholz and Grabowiecki 2007). The application of permeable pavement systems is 
suggested to be limited to parking lots, parking lanes, pedestrian sidewalks, and small alleys that have no or very limited vehicular traffic (District Department of the Environment Watershed Protection Division 2013; MSD 2011). Installation of permeable pavements where the surface slope is excessively deep, or there is great vehicular traffic, may not be feasible (Ferguson 2005).

There are three major types of permeable pavements:

- Porous Asphalt also known as open-graded asphalt, is the standard hot mix asphalt (HMA) with reduced sand or fine materials, which create interconnected void space in the asphalt that allows water drainage through the pavement surface (U.S.EPA 2009a). Porous asphalt, after compaction, contains about 22\% voids (Van Heystraeten and Moraux 1990).

- Pervious Concrete also known as gap-graded concrete, is a concrete with reduced fine materials which create a total void space between 15 to 35 percent in the concrete to allow water drainage from the surface to the storage layer and eventually to the underlying and surrounding soil layers (U.S.EPA 2009b).

- Permeable Pavers consist of individual concrete or stone shapes that are placed adjacent to one another over a specially designed sub-base and allow stormwater to infiltrate into the joints between solid concrete pavers and flow through an open-graded base to underlying soil layers (U.S.EPA 2012c; U.S.EPA 2013b). The gaps between individual concrete pavers are usually, but not necessarily, filled with small-sized aggregates. The 
permeable pavers with aggregates between their joints are referred to as Permeable Interlocking Concrete Pavers (PICP), as shown in Figure 7. The joints and openings typically compromise $5 \%$ to $15 \%$ percent of pavers' surface (U.S.EPA 2009c).

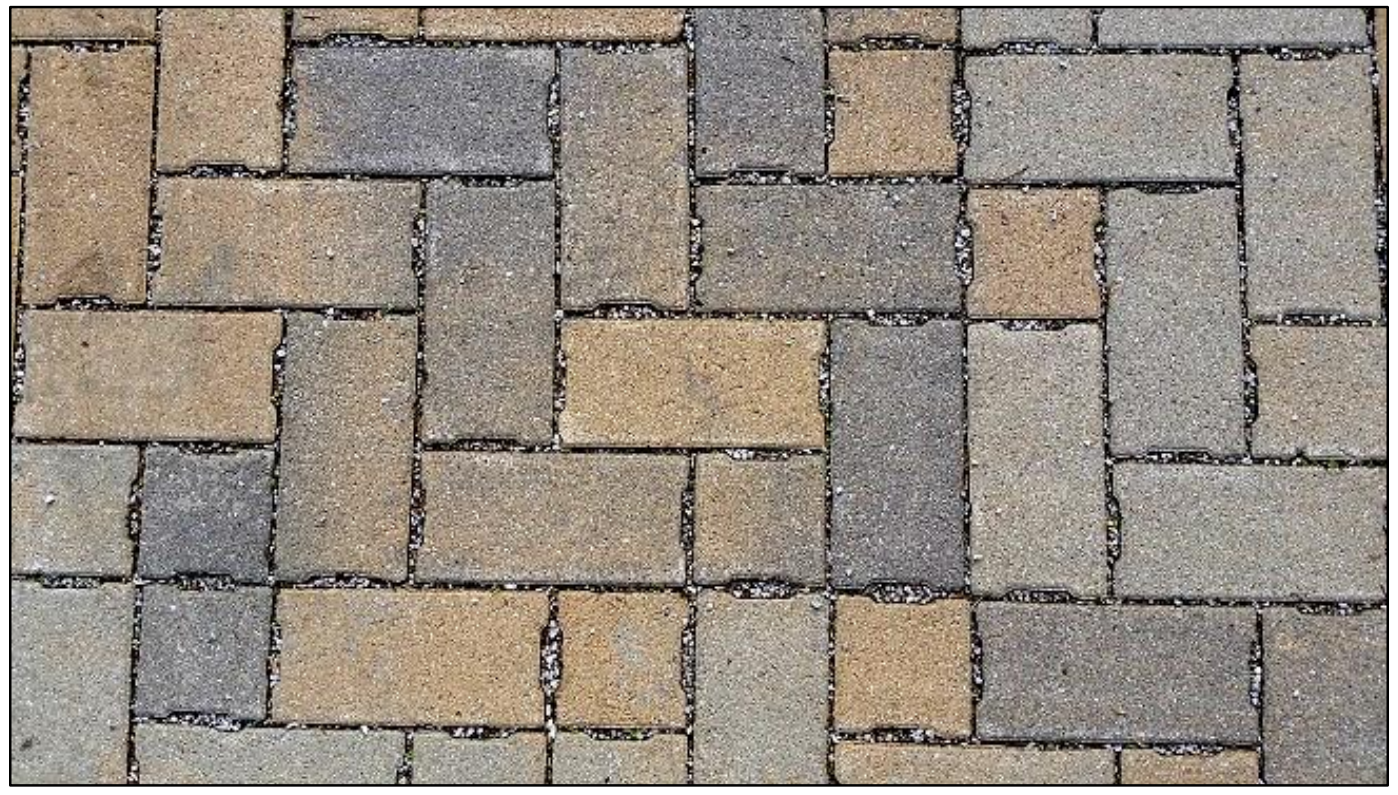

Figure 7 - An Application of Permeable Interlocking Concrete Paver in Louisville, KY

All types of permeable pavements, regardless of the type or brand, accomplish the basic hydrologic goal of infiltration quite well. They do differ, however, in how well they handle high traffic volumes and in their appearances, which should guide their selection by architects and designers (Booth and Leavitt 1999). In a study by Borst el al. (2010) three different permeable pavements surfaces (pervious concrete, porous asphalt, and PICP) were installed in a parking lot and their infiltration rates were measured on a monthly basis, starting at the second month that the parking lot was in use. The first six-month results indicate that the infiltration rates differed significantly from surface to surface. The pervious concrete and porous asphalt had the highest $(4000 \mathrm{~cm} / \mathrm{hr})$ and lowest $(200 \mathrm{~cm} / \mathrm{hr})$ 
infiltration rates (observed unweighted means), respectively. The mean infiltration rates recorded for PICP were $2400 \mathrm{~cm} / \mathrm{hr}$ (Borst et al. 2010).

Bean et al. (2007a) conclude that permeable pavements do considerably reduce runoff, provided the following conditions are met: (1) the pavement is sited in a sandy or loamy sand soil, (2) it is located in soils without seasonally high water tables, (3) the pavement is well maintained, (4) proper construction materials and techniques are used, (5) the pavement is essentially flat and away from disturbed fine soils, and (6) does not have excessive structural loads beyond designed capacity (Bean et al. 2007a).

\subsection{Hydrological Performance of Permeable Pavement Systems}

Previous research studies have found permeable pavements to be very effective in reducing, or even at times eliminating, surface runoff (Bean et al. 2007b; Booth and Leavitt 1999; Brattebo and Booth 2003; Collins et al. 2008; Fassman and Blackbourn 2010). As mentioned before, the hydrological performance of a GI stormwater control measure (SCM) refers to infiltration capacity and exfiltration performance of that GI SCM. In the following section, previous studies regarding these two hydrological functions for permeable pavements are reviewed to provide better understanding of overall hydrological performance of the GI stormwater control and the important contributing factors.

\subsubsection{Infiltration Capacity and Surface Clogging}

Infiltration is a key component mechanism in hydrological performance of GI stormwater controls (Brown and Borst 2013). All permeable pavements are prone to clogging with sediments over time, and thereby slowing their infiltration rates (Bean et al. 2007a; Brown and Borst 2013; Coughlin et al. 2012; Sansalone et al. 2012). According to 
Baladès (1995), the development of clogging is characterized by an increase in the quantity of material retained on the surface, and the clogged area is usually limited to the first $2-\mathrm{cm}$ of the surface structure (Baladès et al. 1995). As the runoff volume infiltrates into the control, the sediments are trapped between permeable pavement's openings and clogging advances on the surface. It is observed that the clogging spreads from the up-gradient edge to down-gradient edge (Brown et al. 2012).

Some studies indicate that clogging develops very rapidly for the first year and then levels off with time (Abbott and Comino-Mateos 2003; Baladès et al. 1995; Bean et al. 2007a; U.S.EPA 2009c). According to U.S.EPA (2009c), while the initial infiltration rates are usually around hundreds of inches per hour, the long-term infiltration capacity remains relatively high even with clogging, and in some circumstance the lowest infiltration rates are still relatively high compared to rainfall intensities (Bean et al. 2007a; Gilbert and Clausen 2006; U.S.EPA 2009c). Even with decreased infiltration rates, possibly due to clogging, the permeable pavement systems result in reduction of runoff volumes (Brattebo and Booth 2003; Gilbert and Clausen 2006). Coughlin at al. (2012) measured clogging by sand and clay in pervious concrete in a laboratory setup. They report that clogging caused by sand or clay decreased the infiltration rates but even after clogging, the infiltration rates were higher than average intensity ( $66 \mathrm{~mm} / \mathrm{hr}$ ) of 100-year 1-hr design storm for Denver, CO (Coughlin et al. 2012).

Each of the studies that investigated the infiltration capacity of permeable pavement systems report a slightly different version of progression of clogging and occurrence of runoff on the surface of permeable pavements. This is believed to be the result of the intrinsic or site-specific characteristics such as: type of the pavement, openings or gap sizes 
on the surface permeable pavement, surface slope, sediment load (or total suspended solids) existent in the stormwater, traffic loadings, and especially the ratio of impermeable surface to the permeable pavement surface, and cumulative captured runoff volume to date (age). These characteristics can affect the clogging pattern and rate. For instance a study by Doe et al. (2010) shows that at a certain ratio of pervious concrete's pore size to particle sizes of the clogging material, the reduction in infiltration rates of pervious concrete due to clogging, is maximum (Deo et al. 2010). Thus clogging rates could be different for permeable surfaces with different gap sizes. So the results of each study must be interpreted with careful consideration of site-specific and intrinsic parameters existent in that specific research.

One of these site-specific characteristics is the sediment load in the stormwater. While the infiltration capacity of a permeable pavement is affected by sediments, it has also been used an indicator of stormwater runoff quality since most of the pollutants in the stormwater are absorbed onto the small sediment particles (Haster and James 1994; Whipple 1983). The amount of sediment in the stormwater that washes onto a permeable pavement system cannot be easily determined until the hydrology of the watershed is investigated. This is because the sediment load in stormwater runoff depends on the volume and rate of storm event at which the runoff occurs (Haster and James 1994).

Slope of the pavement could be another characteristic influencing the clogging of a permeable pavement surface. It has been observed that runoff rates from pervious concrete systems, for similar rainfall intensities, tend to increase with an increase in slope (Valavala et al. 2006). Increase of runoff volume could result in an increase of clogging rate. On the other hand, Fassman and Blackbourn (2010) report that despite the installation 
of a permeable pavement system on high slopes $(6-7.4 \%)$, over 3 years of monitoring revealed exceptional hydrological performance of the permeable pavement systems, well comparable to pre-development conditions. The authors point out that during very intense storm events and on steep slopes, surface runoff may even rise from permeable surfaces (Fassman and Blackbourn 2010).

As mentioned, permeable pavement systems in different environments and under different circumstances would likely perform differently from each other. Two following studies on permeable pavements' performance can be useful in elaborating on this further. In the first study Booth (1999) and Brattebo (2003), monitored four permeable paving systems in a parking lot in Renton, Washington. One of the objectives of the study was to evaluate the long-term performance of these systems, maintenance requirements, and surface clogging. The project site had generally favorable soil conditions (for proper infiltration) and frequent use. The initial results (first year) revealed no measurable surface runoff from the permeable pavement areas (Booth and Leavitt 1999). The authors conclude that long-term (6 years) performance was good in terms of surface durability and infiltration capacity. They report that all permeable pavement systems are apparently as durable as an asphalt surface after 6 years of daily usage and all systems infiltrated all precipitation even during the most intense storm $(7.4 \mathrm{~mm} / \mathrm{hr})$ recorded during the study period. Although the authors eventually note that Pacific Northwest area has generally low rainfall intensities and their positive results may not apply to other areas with higher intensity rainfall events. Surface deposits were observed but the decrease of infiltration capacity wasn’t significant (Brattebo and Booth 2003). 
In the second study, Bean et al. (2007a) conducted surface infiltration tests for 40 sites with permeable pavement systems that included PICP, porous concrete and concrete grid pavers (CGP). The test sites were located in North Carolina, Maryland, Delaware, and Virginia. Before and after conducting maintenance treatments, two series of surface infiltration tests were conducted on the CGP. The surface infiltration measurements were conducted on 15 CGP sites, and 14 of them showed higher infiltration rates after the maintenance treatments. Fourteen PICP sites were tested and the results showed that surface infiltration rates of sites located adjacent to disturbed soil, or that had fines deposited on them, were significantly lower than those rates from sites away from fines. They report a median surface infiltration rate of $8(\mathrm{~cm} / \mathrm{hr})$ for sites clogged with fines which shows more than $99 \%$ overall decrease when compared to infiltration rate of $2000(\mathrm{~cm} / \mathrm{hr})$ for sites free of fines. The testing results from PC sites show the same decrease in infiltration rates between clogged and unclogged sections. They conclude that installing permeable pavement systems away from disturbed soil areas is a significant factor in maintaining higher infiltration rates for the pavement and also that the maintenance is key to sustain high surface infiltration rates (Bean et al. 2007a). By comparing these two studies it appears that climate conditions and environment of the project site could greatly affect the infiltration and overall performance of permeable pavement systems.

The other factor associated with clogging rate is age. As mentioned earlier, the overall surface clogging is expected to increase with the age of clogging and no substantial clogging is usually observed for newly constructed permeable pavements. Collins et al. (2008) monitored the performance of four permeable pavement sections, including pervious concrete (PC), two types of PICP, and concrete grid pavers (CGP), for 
hydrological differences in pavement surface runoff volumes, total outflow volumes, peak flow rates, and time to peak. They report that all pavement sections significantly reduced surface runoff volumes and peak flow rates. They observed no substantial clogging over the course of the study (one year) (Collins et al. 2008).

On the other hand, evaluation of the long-term performance of two porous asphalt installations in northern Sweden, aged 18 and 24 years, showed a significant decrease in infiltration capacity of both systems due to visible surface clogging (Al-Rubaei et al. 2013). In another study infiltration rates were measured on 20 pervious concrete pavements in California. The results showed a significant variability in measured infiltration rates within each parking lot and between different parking lots. The authors report that age is the predominant factor influencing the infiltration rates of permeable pavements (Kayhanian et al. 2012).

In summary the following has been reported by different researchers regarding the clogging and infiltration capacity of permeable pavement systems (Abbott and CominoMateos 2003; Al-Rubaei et al. 2013; Bean et al. 2007a; Booth and Leavitt 1999; Brattebo and Booth 2003; Collins et al. 2008; Coughlin et al. 2012; Fassman and Blackbourn 2010; Kayhanian et al. 2012):

- Permeable pavement systems are effective in managing surface stormwater runoff due to their high infiltration capacities, and when compared to impermeable surfaces, they can significantly reduce the surface runoff volume and peak runoff rates. 
- Surface clogging is a substantial factor affecting the infiltration capacities of permeable pavements, which is caused by accumulation of fine sediments in surface openings and gaps of permeable pavement systems. While some studies show a decrease in surface infiltration rates of permeable pavement systems, others report no observed clogging, and subsequently no decrease in surface infiltration capacities.

- Even after clogging, permeable pavements manage to keep a fraction of their initial infiltration capacity. This would imply that even a clogged permeable pavement is likely able to capture the rainfall that falls on its surface area.

- Age and location of the permeable pavement systems are the predominant factors that affect the surface clogging and subsequently the infiltration capacity.

\subsubsection{Surface Maintenance Treatments}

The most prevalent maintenance concern for permeable pavement systems is the potential clogging of the pervious concrete pores. As the stormwater infiltrates into the permeable pavement surface, the fine materials and sediments existing in the surface runoff are trapped between the pores and openings of the pavement surfaces. Clogging will increase with age and use (U.S.EPA 2009a; U.S.EPA 2009b; U.S.EPA 2009c). The Clogging limits the infiltration performance of permeable pavement systems, but can be restored by cleaning and maintaining of the surface (Baladès et al. 1995). Clogging is more easily removed shortly after forming and before the fine sediments are compacted or migrated to the voids at lower depths, which are harder to clean (Bean et al. 2007a). 
Different studies report different surface maintenance methods and results in restoring the infiltration capacity of permeable pavements, but in general they show that an effective surface maintenance treatment can partially, or even fully, restore the initial infiltration capacity (Al-Rubaei et al. 2013; Bean et al. 2007a; Sansalone et al. 2012). Different surface cleaning methods investigated in previous studies include sweeping, vacuuming (or suction), using high pressure water jet, sonication, or a combination of these methods.

A combined high pressure washing and vacuum cleaning method was used to restore the infiltration capacity of two clogged porous asphalt systems, aged 18 and 24 years. The applied method was able to partially restore the infiltration rates for 18-year-old porous asphalt but had no effect that on the other (24-year-old) system (Al-Rubaei et al. 2013). Fassman and Blackbourn (2010) also report that pressure washing successfully restored the infiltration rates of a permeable pavement system (PICP) by more than an order of magnitude. It must be noted that pressure washing could result in washing the sediments into the system and eventually polluting the receiving waters (Chopra et al. 2010; Fassman and Blackbourn 2010). The pressure washing was also used on pervious concrete systems that were clogged by clay and sand in a laboratory study. The results show no significant increase on the infiltration rates of pavements, despite the visual inspection that implied otherwise (Coughlin et al. 2012). Sansalone et al. report that vacuuming and sonication both can be used as effective maintenance methods in recovering the surface infiltration rates of permeable pavement systems (Sansalone et al. 2012). Chopra (2010) investigated the effectiveness of different maintenance methods in restoring the infiltration capacities of clogged pervious concrete sections. The methods used in this study included vacuum 
sweeping, pressure washing, and vacuum sweeping followed by pressure washing. The results show that pressure washing is more effective than vacuum sweeping and a combination of both appears to be the most effective (Chopra et al. 2010).

Results of various studies indicate that surface infiltration capacities of permeable pavement systems can partially or fully be restored, with selection of right cleaning method (Baladès et al. 1995; Bean et al. 2007a; Chopra et al. 2010; Fassman and Blackbourn 2010). The effectiveness of these methods mostly depends on how clogged the permeable pavements are and which surface maintenance method is selected (Baladès et al. 1995). Regular maintenance treatments are suggested as the most important measures in retaining the long-term infiltration capacity of permeable pavements (Al-Rubaei et al. 2013).

\subsubsection{Exfiltration Performance and Contributing Factors}

In order to ensure proper hydrological performance of stormwater control measures, the exfiltration performance, as well as infiltration capacity, needs to be monitored. A satisfactory combined performance of these two hydrological functions is essential to ensure effective stormwater management by use of GI stormwater control measures.

There are limited past studies, investigating the exfiltration performance of the GI stormwater control measures, especially its long-term performance. This is mainly because most of past studies were focused on redirecting the captured stormwater to collection tanks to determine the outflow volume, instead of allowing it to naturally exfiltrate to surrounding and underlying soil layers (Collins et al. 2008). 
Chai et al. (2006) conclude that saturated hydraulic conductivity of the underlying soil is the most important factor that must be considered in the design of permeable pavements (Chai et al. 2012). Implementation of permeable pavement systems is recommended on soil layers that have adequate permeability and therefor suitable infiltration capacity (Booth and Leavitt 1999; District Department of the Environment Watershed Protection Division 2013; MSD 2011). Valavala et al. (2006) studied runoff coefficients of unclogged pervious concrete systems which were placed on 15 -cm deep layer of sand. The authors conclude that permeability of the sand layer can be a limiting factor for pervious concrete systems (Valavala et al. 2006). Other authors also mention that infiltration performance of a permeable pavement system is limited by the rate of exfiltration to the subgrade, especially for unclogged sections (Coughlin et al. 2012; Haselbach et al. 2006). The low values of permeability (or hydraulic conductivity) of the porous media is not only a limiting factor in exfiltration performance of permeable pavement systems, but these low permeability soil layers are also more prone to permeability damage caused by solid particles than high permeability soil layers (Moghadasi et al. 2004). Solid particles may already be present in the system (e.g. attached to storage layer aggregates of the permeable pavement system), or brought into it with the captured stormwater runoff (e.g. total suspended solids in stormwater). These particles are either deposited at the bottom of the storage layer or stuck in the pores of the soil layers, causing reduction in exfiltration rates and subsequently exfiltration performance of the system.

There are also studies that report satisfactory performance of GI controls even on fine grained and clayey soils. Dreelin et al., (2006) monitored the performance of 
permeable pavement parking lot in Athens, Georgia that was constructed on rich-clay soils. They report that permeable parking lots effectively decreased the amount of runoff for storm events with less than $2 \mathrm{~cm}$ total depth (Dreelin et al. 2006). Fassman et al. (2010) also report that despite installation of permeable pavement system over relatively impermeable subgrade soils, the overall hydrologic performance of the permeable pavement was exceptional (Fassman and Blackbourn 2010).

Pitt et al. (2008) did a study to correlate the compaction of soil layers with infiltration rates by conducting laboratory and filed tests. They conclude that as expected, the degree of compaction greatly affected the infiltration rates for sandy and clayey soils. Antecedent moisture conditions were an important factor for clayey soils but had a minimal effect on sandy soils (Pitt et al. 2008).

Changes in temperature also cause changes in hydraulic conductivity values. This is attributable to temperature effects on the viscosity of water and the effect of the viscosity changes on hydraulic conductivity values. The results of a systematic long-term study of infiltration rate in a large scale effluent recharge plant, shows a significant dependence of the infiltration rate on temperature. In this investigation the infiltration rate values were studied for seasonal changes over a 4-year period. Lin et al. (2003) also found that the temperature effects on infiltration rate tend to be larger by a factor of 1.5-2.5 times than the change expected from effluent viscosity changes alone. The increase in hydraulic conductivity with increase in temperature is commonly attributed to the decrease in water viscosity. This effect was also reported in a number of laboratory studies; however, the magnitude of the change differed considerably among the reports and in some cases 
hydraulic conductivity changed by orders of magnitude more than predicted from viscosity change alone (Lin et al. 2003).

Braga et al. (2007) reported that for water level depths less than 10-cm, hydraulic conductivity is the most sensitive parameter influencing exfiltration rates. The authors remark that higher temperatures, which occur during warmer periods, can affect the exfiltration rate by as much as $56 \%$ (Braga et al. 2007).

To further assess the effect of temperature on GI performance, two infiltration GI controls on campus of Villanova University were monitored for 4 and 2 years to evaluate their long-term and seasonal variations of hydrological performances. The GI controls had drainage ratios (impervious area to GI control's area) of 3:1 and 10:1. While the results didn't show any significant degradation in exfiltration performance they both show seasonal variations which are explained by temperature dependency of the viscosity of water. However the GI control with coarser underlying soil layers, and obviously higher hydraulic conductivity values, showed stronger temperature dependency compared to the other GI control with finer underlying soil layers. The authors deduce that this phenomena is because of the higher intrinsic permeability of courser media which makes it more sensitive to changes in the fluidity of water (Emerson and Traver 2008).

Another study was conducted on long-term performance of a 1.8-m deep infiltration trench with an impervious drainage area to GI control's area of approximately 160:1. The high drainage ratio was intentional to accelerate the longevity-related processes. A drastic change in exfiltration performance of the GI control was observed over the 3-year course of the study. The authors conclude that the bottom of the GI control was likely clogged 
with sediments which resulted in negligible exfiltration through the bottom of the control while the exfiltration through the lateral areas of GI control didn't show any significant changes during the three year period. Seasonal variations were also observed which even reversed the decreasing trend of exfiltration performance over summer times (Emerson et al. 2010). It appears that other factors other than temperature can also affect the exfiltration performance of GI controls.

LeBoon and Traver (2007) mention that factors such as moisture content, and depth of ponded water also affect infiltration rates of soil layers (LeBoon and Traver 2007). Extra care must be taken in interpreting the results of studies that investigate the effects of these contributing factors as each study has some specific characteristics that could easily be ignored. Among such characteristics are climate conditions (mostly rainfall patterns and its characteristics) of the region that the study is carried out in and also the drainage ratios (ratio of impervious area draining into the GI control to its surface area) for each GI control. Next section will briefly review the effects of rainfall characteristics on hydrological performance of Stormwater Control Measures.

\subsubsection{Rainfall Characteristics of Storm Events}

Precipitation is dynamic in nature, as it not only varies from one location to another, but it also has a varied pattern in a specific location. The falling rate of rain is called intensity (intensity = rainfall depth/duration) usually expressed by inches per hour (in/hr), or millimeters per hour $(\mathrm{mm} / \mathrm{hr})$. The rainfall intensity depends on the duration of the rainfall and the frequency of rainfall event. The less frequent the rainfall event, the larger its intensity (Pazwash 2011). 
The instantaneous rainfall intensity varies with time and during a rainfall event. To simplify analysis of a rainfall, the storm period is divided into segments and average rainfall intensity is calculated for each segment (Figure 8).

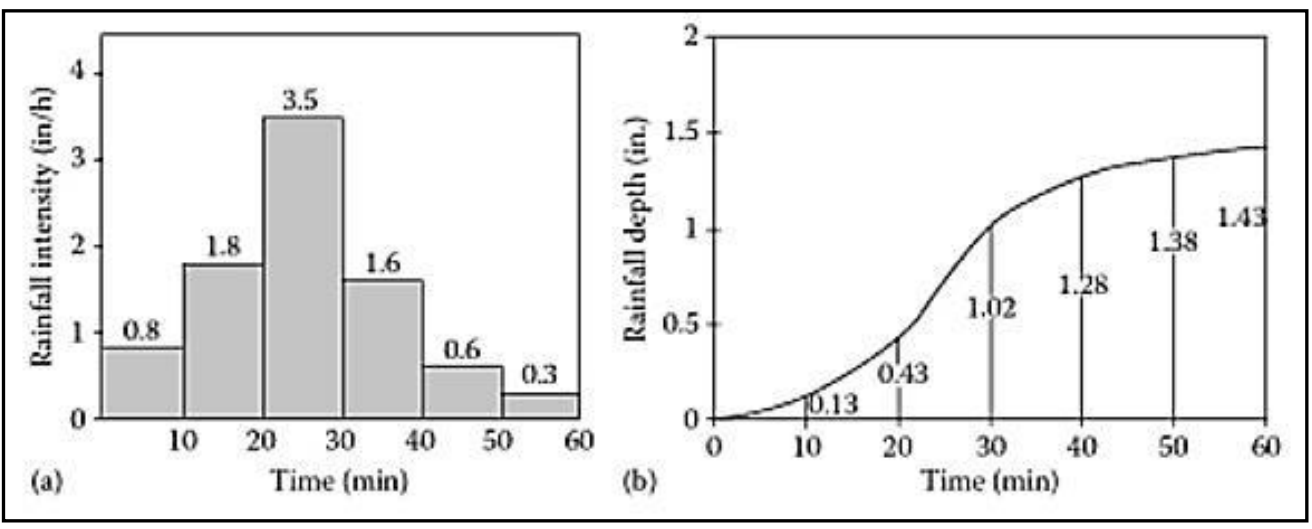

Figure 8 - Example of a rainfall hyetograph (a), and cumulative rainfall depth (b) (Pazwash 2011)

Effects of rainfall depth and intensity on performance of GI stormwater controls has been investigated by different researchers. Pyke et al. studied (2011) the effects of rainfall characteristics on stormwater runoff volume. The results show that stormwater runoff is more sensitive to changes in precipitation volume than event intensity (Pyke et al. 2011).

Hou et. al., (2008) studied the effects of rainfall intensity on runoff volume from permeable pavements. They mention rainfall intensity as a predominant factor in causing runoff. Their results show that permeable pavement surfaces delayed the onset of surface runoff, and higher intensities of rainfall resulted in faster and higher runoff rates (Hou et al. 2008).

Collins et al. (2008) conclude in their study, that the rainfall intensity was the best predictor of surface runoff from permeable pavements, and time to peak flow. Unlike 
surface runoff, total outflow volume was mostly affected by rainfall depth than rainfall intensity. It is suspected that the effects of rainfall intensity were lost, as water traveled through filtration layers which probably had a dampening effect on the surface runoff flows. The authors report that in general, out flow volumes were greater during months of fall and winter (Collins et al. 2008). Fassman et al. (2010), also report that rainfall intensities have a positive relationship with runoff flow rates from impermeable asphalt, and while the outflow volumes from permeable pavement surfaces were also positively related to intensity and depth of the rainfall, less variations were observed compared to runoff from asphalt (Fassman and Blackbourn 2010).

As see in literature, the rainfall parameters such as depth, duration, and intensity could greatly affect the hydrological performance of a GI control. Complete understanding and effective measurement of these parameters is critical as monitoring results must be interpreted with respect to these parameters.

\subsection{Summary and Conclusions}

In this chapter the diffuse literature on hydrological performance of permeable pavers were reviewed and summarized. As discussed two primal functions include the hydrological performance of a permeable pavement system: infiltration capacity and exfiltration performance. Both of these functions are affected by clogging, either at the surface or at the interface of the storage layer and underlying soil layer. Clogging at surface happens as fine sediments are trapped in the gaps, openings, or the pore structure of the permeable pavement system and causes reduction in infiltration capacity of the system. Maintenance treatment methods can be applied to restore the infiltration capacity. The 
effectiveness of the maintenance effort depends on the age of the control, cleaning method, and the type of pavement.

Exfiltration performance of GI controls is primarily affected by hydraulic conductivity values (infiltration rates) of underlying and surrounding soil layers which depend on factors such as: the hydraulic head (or depth of ponded water), antecedent moisture conditions, and temperature. While such factors could have cyclic variation over time for a specific location, there are other factors that are mostly site specific and aren't usually expected to change over time in a single location, such as soil type and compaction levels of soil layers. The accumulation of sediments at the interface of storage layer and underlying soil layers is another factor that is expected to increase with the age of the GI control and result in clogging and decreased exfiltration performance of the GI control. It must be noted that results of a previous research project may not be applicable to other locales because of the difference in site-specific characteristics for different areas. Among which are precipitation characteristics of an area such as rainfall depth, duration, and intensity. Any investigation that tends to effectively monitor the hydrological performance of the GI controls needs to consider, understand, and efficiently measure the rainfall characteristics of that location.

To ensure proper hydrological performance of a GI control, effects of influencing factors on infiltration capacity and exfiltration performance must be known and considered during the design process of these controls. This study intends to investigate the hydrological performance of two permeable pavement practices in Louisville over a 2-year period, by consideration of potential contributing factors that were reviewed in this chapter. 


\section{EXPERIMENTAL APPROACH}

\subsection{Introduction}

The project background was briefly reviewed in the first Chapter of this document. As mentioned, in April 2009, the Louisville and Jefferson County Metropolitan Sewer District (MSD) entered into a consent decree with the US Department of Justice, the United States Environmental Protection Agency (EPA) and the Kentucky Department for Environmental Protection (KDEP) to decrease the number and volume of untreated sewer overflows entering its combined sewer system. To accomplish this goal, the MSD developed an Integrated Overflow Abatement Plan (IOAP). This plan includes a mixture of Green Infrastructure (GI) and Gray solutions to control the wet weather CSOs and approximately 17 percent of its budget is allocated for GI solutions (MSD 2013a; MSD 2013b). The MSD is committed to develop and implement a monitoring plan to evaluate the performance of various green infrastructure elements, so the knowledge gained from this monitoring effort can be used as a decision making factor in the future implementations of GI controls in Louisville (MSD 2009).

One of the initial phases of this project includes implementing a set of GI stormwater controls in CSO130 sewer-shed, which is located in the Butchertown neighborhood area, at the East of Louisville. This sewer-shed is 11 hectares (28 acres) and the MSD planned to mitigate the CSO problem in this area, to objectives defined in the IOAP, only by use of GI stormwater controls. 
The MSD and the URS Corporation (URS) were responsible for design and implementation of the GI controls throughout the CSO130 basin and the EPA's office of Research and Development (EPA) and the Center for Infrastructure Research (CIR) at the University of Louisville (UofL) were tasked with preparing a monitoring plan, which is aimed to evaluate the individual performances and overall effectiveness of the GI controls in mitigating the CSO problem in the CSO130 sewershed. The overflows from this basin is discharged to Beargrass Creek which flows into the Ohio River, see Figure 9.

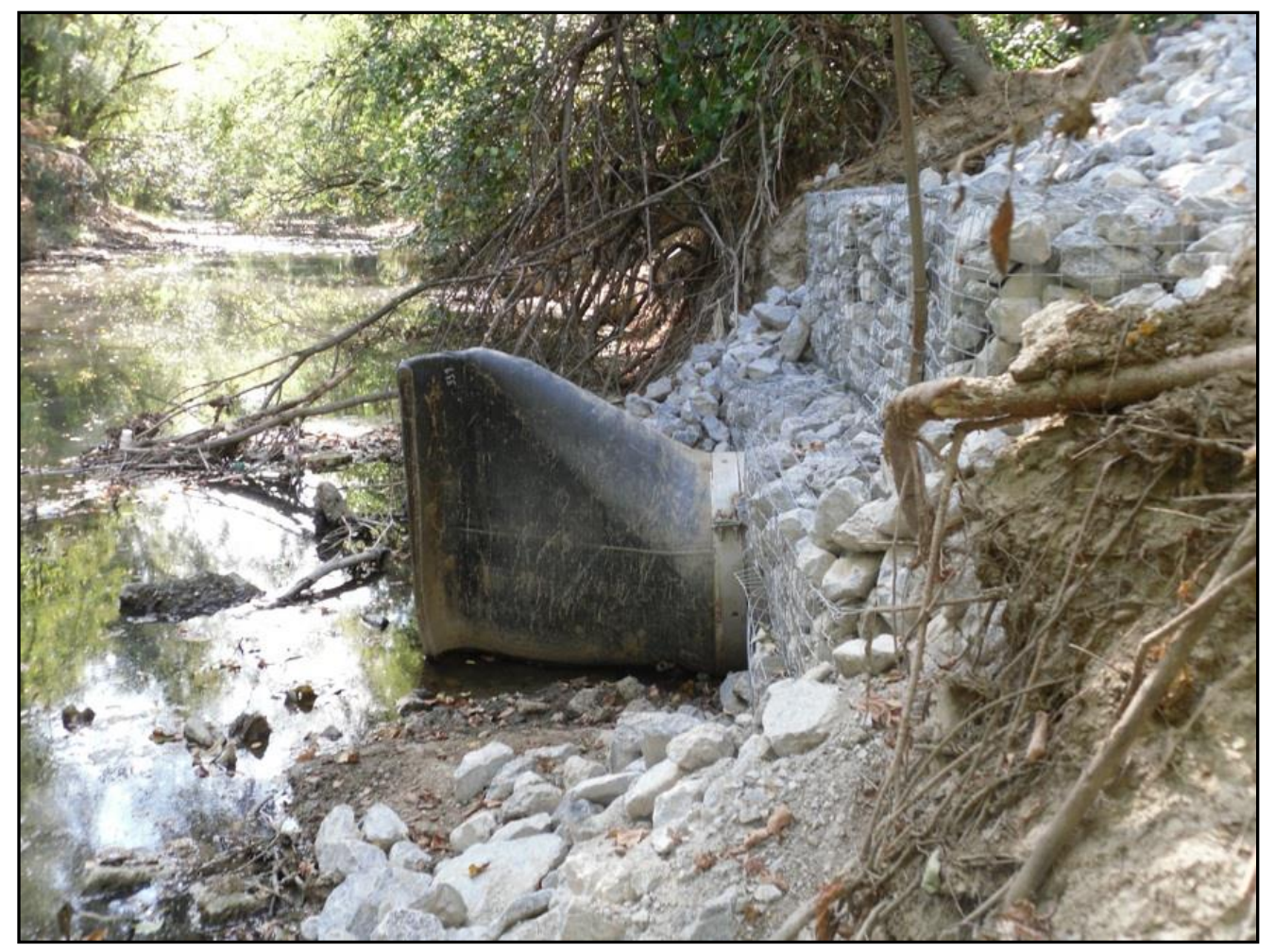

Figure 9 - Discharge Point of CSO130 to Beargrass Creek (Badwaterjournal.com 2010)

A total number of 18 permeable pavement strips and 29 treeboxes were designed to capture the surface runoff volumes from storm events, see Figure 10. All the proposed GI stormwater controls were placed along Butchertown area's streets/sidewalks and upgradient of the existent catch basins to capture the surface runoff before entering the sewer 
system. Since the MSD intended to implement all these GI controls in publ icly owned areas to have control over future maintenance treatments, the permeable pavement systems were designed for installation along the existing street parking lanes. The permeable pavement strips ranged from $55 \mathrm{ft}$ to $120 \mathrm{ft}$ and were 8-ft wide. The MSD's objective was to decrease the volume and quantity of overflows in this basin to approximately half of their original values for the design rainfall year ${ }^{1}$, see Table 1.

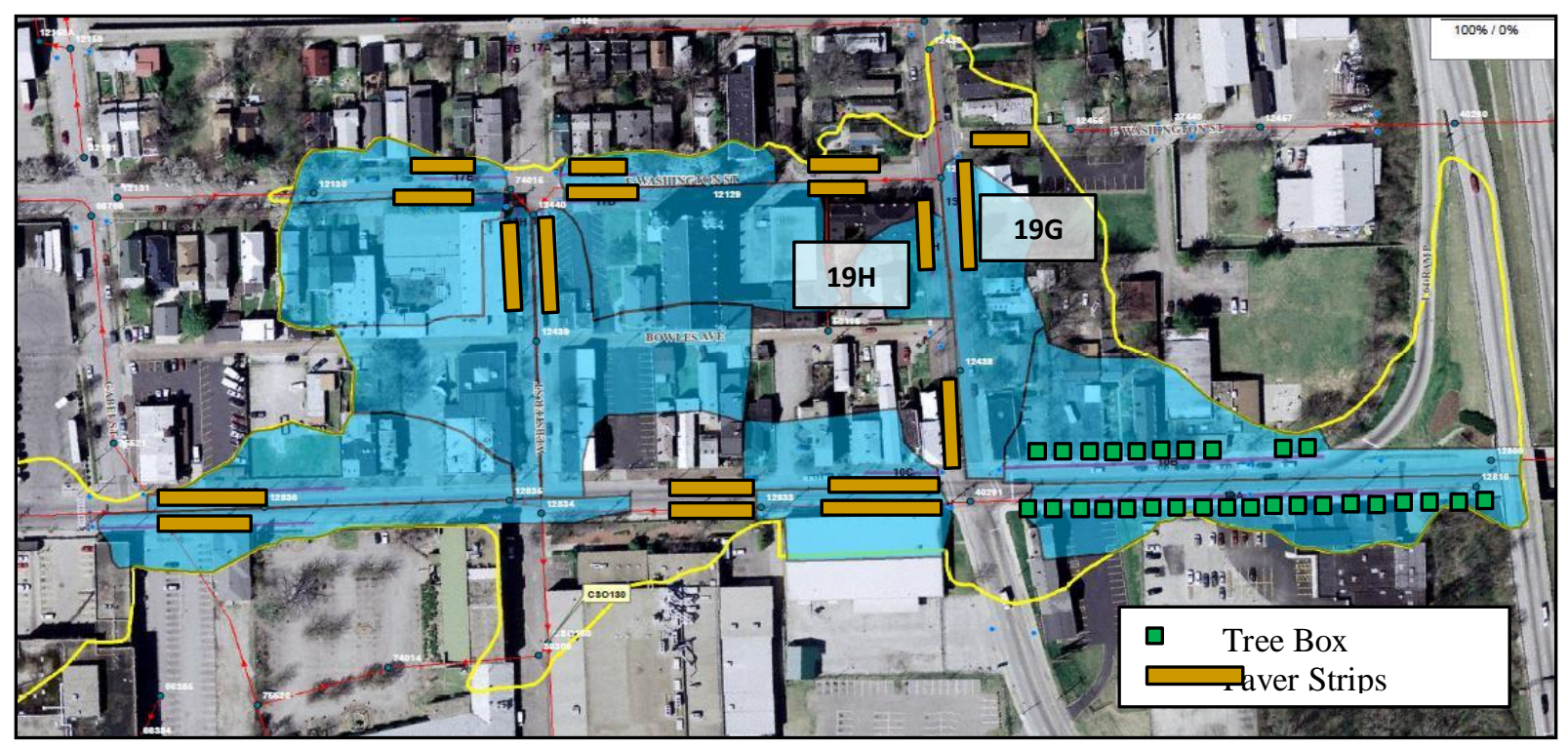

Figure 10 - Proposed GI controls for CSO130

In November and December 2011, two of these permeable pavement strips were constructed along the parking lanes of Adams Street and the rest of the controls were installed during 2013. This study investigates the hydrological performances and their associated processes of the first two installed GI stormwater controls since their implementation through the end of 2013. 2001.

\footnotetext{
1 - The design rainfall year, used during the designing of GI controls in CSO130, is rainfall year
} 
Table 1 - The MSD's Objectives for CSO Mitigation in CSO130

\begin{tabular}{lllc}
\hline Metric & Current & Target & Reduction \\
\hline $\begin{array}{l}\text { Annual Average Overflow } \\
\text { Volume (AAOV) (MG/Yr) }\end{array}$ & 1.3 & 0.67 & $52 \%$ \\
\hline Overflows & 16 & 8 & $50 \%$
\end{tabular}

\subsection{Project Description}

The MSD intended to mitigate the CSO problem in CSO130 watershed by the use of GI stormwater controls. The initial phase of this project started by installation of GI controls 19G and 19H in November and December 2011. The GI controls were designed in form of permeable pavement strips which were installed along the parking lanes of Adams Street and at the up-gradient of existing catch basins. The characteristics and dimensions of both controls is provided in Table 2.

Table 2 - Characteristics of Control 19G \& 19H

\begin{tabular}{lll}
\hline Characteristic & Control 19H & Control 19G \\
\hline Drainage Area & 0.11 ha & 0.29 ha \\
\hline Impervious \% & $59 \%$ & $61 \%$ \\
\hline Impervious Area: Control's Area & $15.8: 1$ & $20: 1$ \\
\hline Control's Length & $16.8(\mathrm{~m})$ & $36.6(\mathrm{~m})$ \\
\hline Control's Width & $2.44(\mathrm{~m})$ & $2.44(\mathrm{~m})$ \\
\hline
\end{tabular}

The surface of both GI control were paved with articulated concrete blocks (ACB), see Figure 11, which covered a 0.91-cm (3-ft) deep storage gallery. Along the center of the storage gallery a $3.05-\mathrm{m}(10-\mathrm{ft})$ deep trench was excavated to provide extra storage volume and more importantly, access to soil layers with higher hydraulic conductivity values. The trenches were off centered to avoid the existing utility lines. Figure 12 shows the cross 
sections of controls $19 \mathrm{G}$ and $19 \mathrm{H}$. The ACBs' open area is approximately $3 \%$ of their total surface area (PaveDrain 2011).

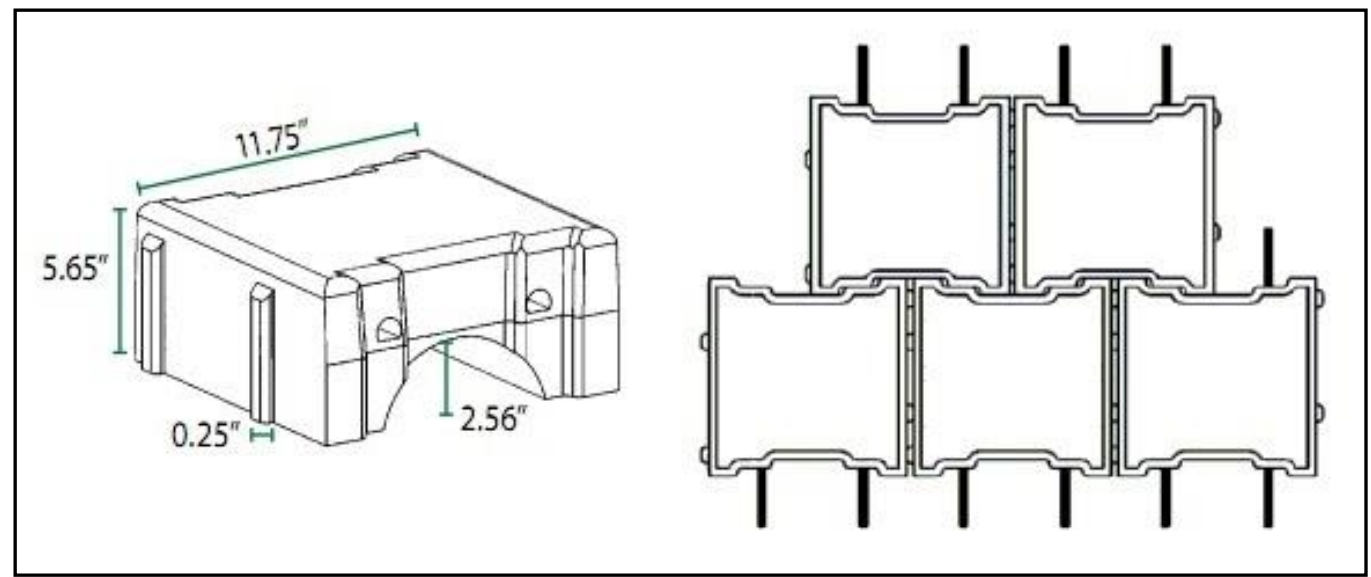

Figure 11 - Dimension and Setup of Articluated Concrete Pavers (PaveDrain 2011)

As shown in Figure 12, the ACPs are placed over an 18-inch (45-cm) thick layer of AASHTO ${ }^{1} \# 57$ bedding stone. The remaining depth of the storage gallery and trench are filled with AASHTO \#3 stone. A geo-grid is placed at the interface of \#3 stone and \#57 stone layers.

Both controls include a 6-in $(15-\mathrm{cm})$ perforated ductile iron overflow pipe at downgradient edge, and placed in \#57 layer, to discharge the captured runoff to sewer system, in case the accumulated water exceeds the capacity of the storage layer.

\footnotetext{
${ }^{1}$ American Association of State Highway and Transportation Officials
} 


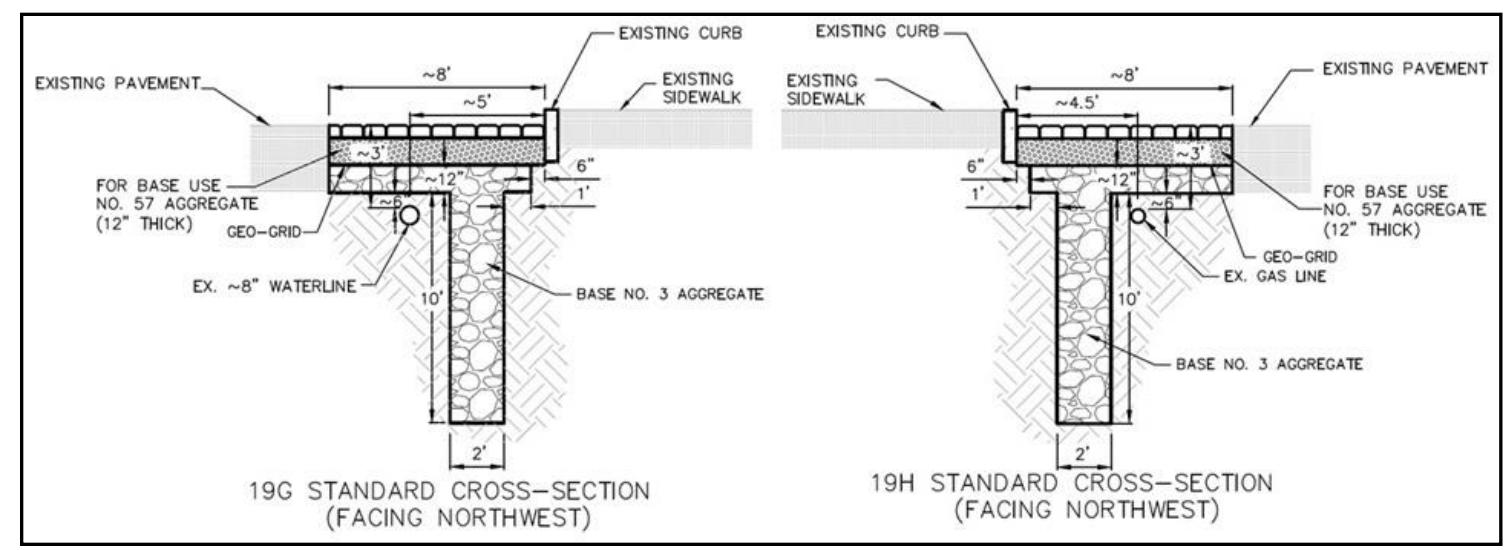

Figure 12 - Cross Sections of GI Controls 19G (left) and 19H (right)

The different stages of the construction and finished permeable pavement systems are shown in Figure 13. As part of the monitoring effort, the EPA and UofL were present during the construction steps at all times to install the monitoring sensors and conduct insitu tests. 


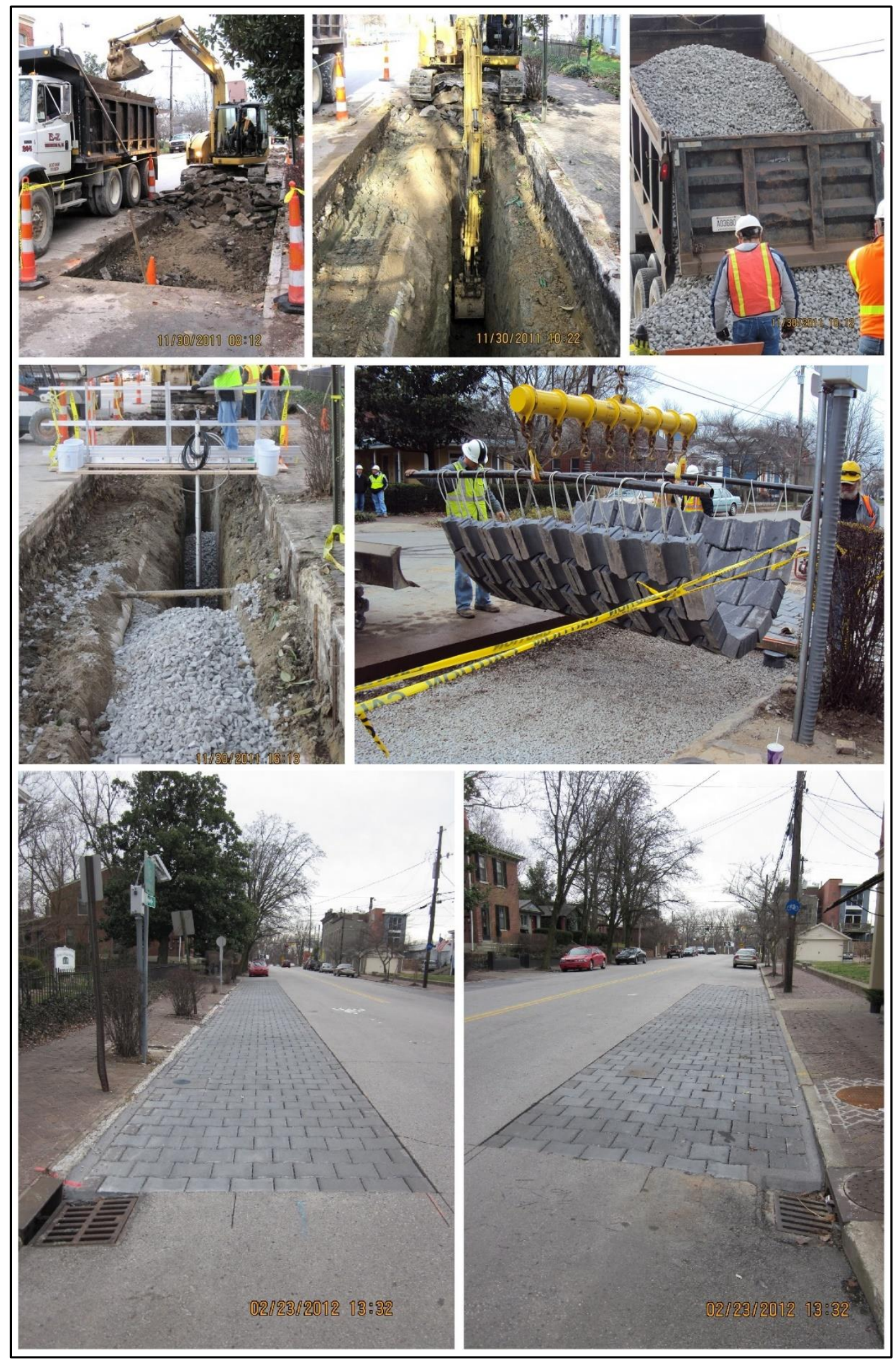

Figure 13 - Construction Stages of Controls 19G \& 19H 


\subsection{Monitoring Plan}

Louisville MSD worked with the EPA and UofL to develop and implement a monitoring effort that would assess the effectiveness of individual GI stormwater controls in CSO130. The two controls, 19G and 19H, were among the GI controls selected for individual performance monitoring. Each GI control's individual performance monitoring included both electronic and manual measurements. The manual tests can be grouped into two categories: 1) pre-construction tests/measurements conducted to assess the properties of the underlying soil layers and different components of GI controls, and 2) tests/measurements conducted periodically during the service lives of each GI control. The electronic measurement utilized data collected through embedded piezometers (pressure transducer type), time domain reflectometers (TDRs), and thermistors which were installed during the construction.

The overall effectiveness of GI stormwater controls in diverting runoff from combined sewer system is evaluated through measurements collected by MSD's sewer flow monitoring network. The MSD installed flow meters (total of five, area-velocity type) to measure the flow in the sewer lines and at the CSO's discharge point.

During this phase of the monitoring effort, the rainfall data within the sewershed is estimated by using one of the MSD's rain gauges which is installed approximately $1-\mathrm{km}$ from the test site and also through the RADAR data provided by the MSD. All rainfall measurements are recorded at 5-minute intervals. 


\subsubsection{Instrumentation}

As mentioned, the electronic sensors installed in controls $19 \mathrm{G}$ and $19 \mathrm{H}$ include pressure transducers, TDRs, and thermistors. This section explains the placement of these sensors and their anticipated role in evaluation of performance of each GI control.

Infiltration of the surface runoff and progression of surface clogging is monitored by an array of TDRs installed just on top of the \#3 stone layer, which is approximately 1.5 $\mathrm{ft}(75 \mathrm{~cm})$ below the pavers. TDRs are also installed at the bottom of the trench to enable measurement of the time that it takes for the captured runoff to reach that point. TDRs measure the volumetric water content (VWC), by measuring the dielectric permittivity, of the media that they are installed in and are mostly used in agricultural applications where this media consists of relatively fine soils. In this project, since the TDRs are placed in \#3 and \#57 aggregates, it is not expected of them to report true values of VWC but they are used to compare changes in infiltration behavior between different locations and over time. The selected TDRs in this project are Model CS650 produced by Campbell Scientific Co., see Figure 14. The CS650 TDRs have two tines that are 12-in (30-cm) long.

The array of TDRs, at the interface of \#3 and \#57 stone layers, are installed at three constant distances from the curb side: at 6 in. $(0.15 \mathrm{~m})$, at 48 in. $(1.22 \mathrm{~m})$, and at 90 in $(2.29$ m). Since it was expected to see the up-gradient side clog first, the first four TDRs were placed logarithmically in first 40-ft length of the control, and the remaining TDRs were placed within the fixed 40-ft spacing, see Figure 15. Since it was expected for the majority of the runoff volume to flow along the curb side, the TDRs are concentrated along the curb and middle side. Since Figure 15 shows the TDRs used in control 19G (120-ft long), control 19H (55-ft long) only included the first 10 TDRs from the up-gradient edge. Three TDRs 
were also installed at the bottom of trench in each GI control. These TDRs were placed on the underlying soil surface and then covered with \#3 stone.

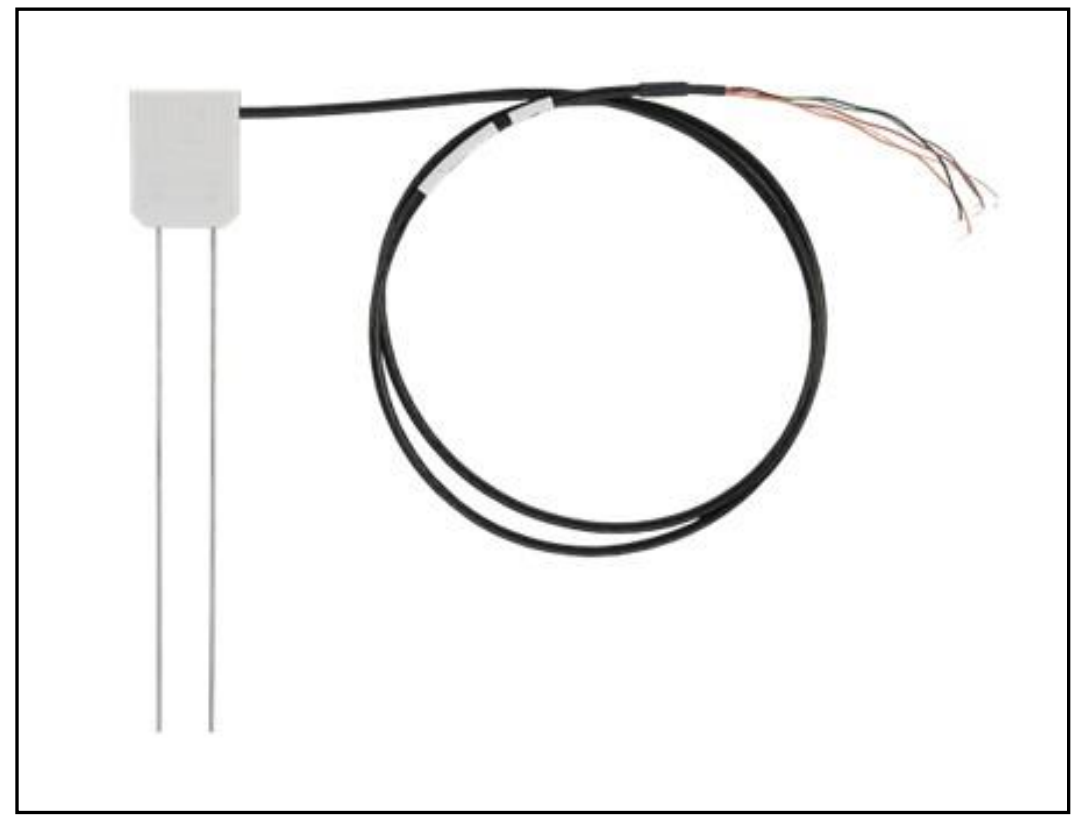

Figure 14 - TDR, Campbell Scientific Model CS650 (image source: Campbell Scientific Co.)

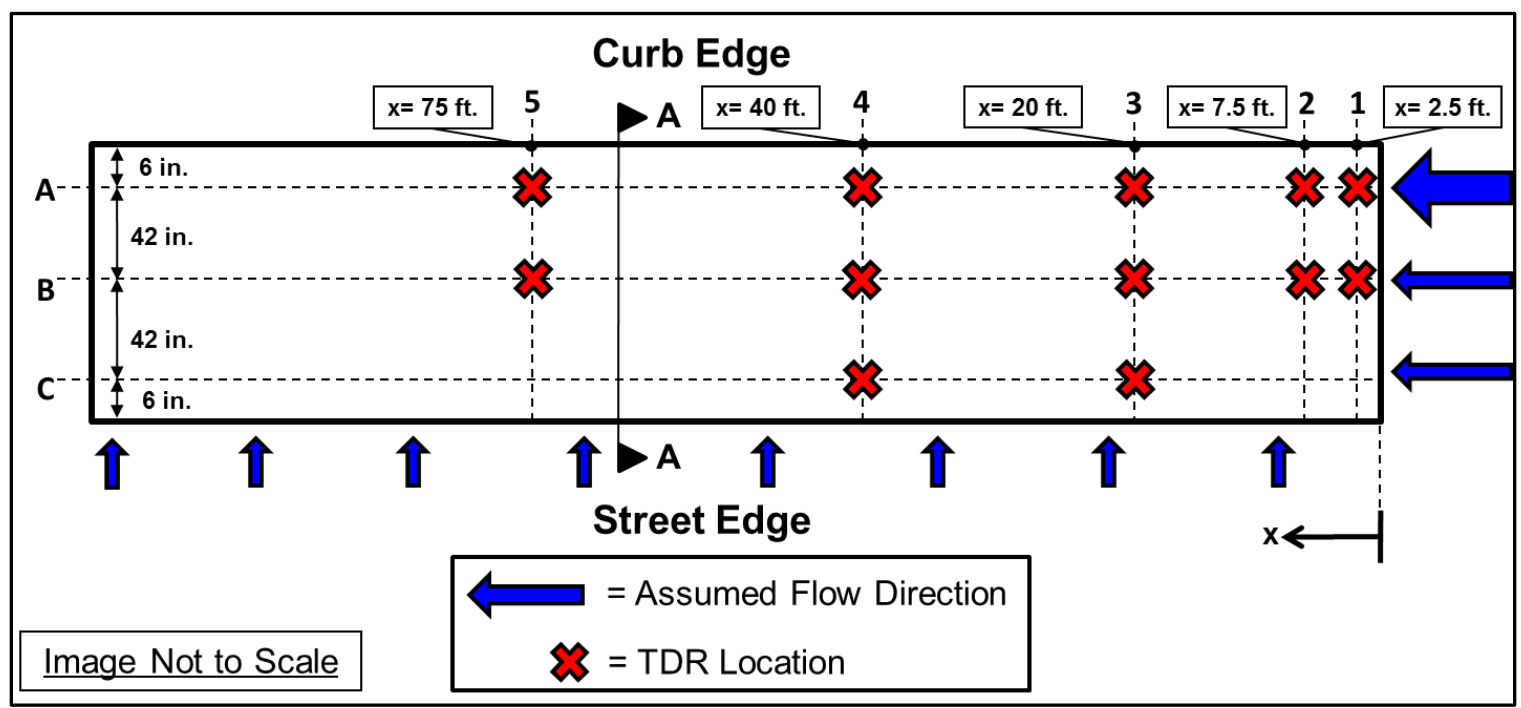

Figure 15 - Plan View of TDRs Placement at the Interface of \#3 and \#57 Stone Layers 
Three pressure transducers were also installed at the bottom of the trench in each control. The pressure transducers were used to measure the captured water level inside the GI control. The model used was Model CS450 produced by Campbell Scientific Co., see Figure 16. These pressure transducer had a range of $0-5.1$ meter and accuracy of \pm 0.0051 meter. The pressure transducers and bottom TDRs were positioned adjacent to each other.

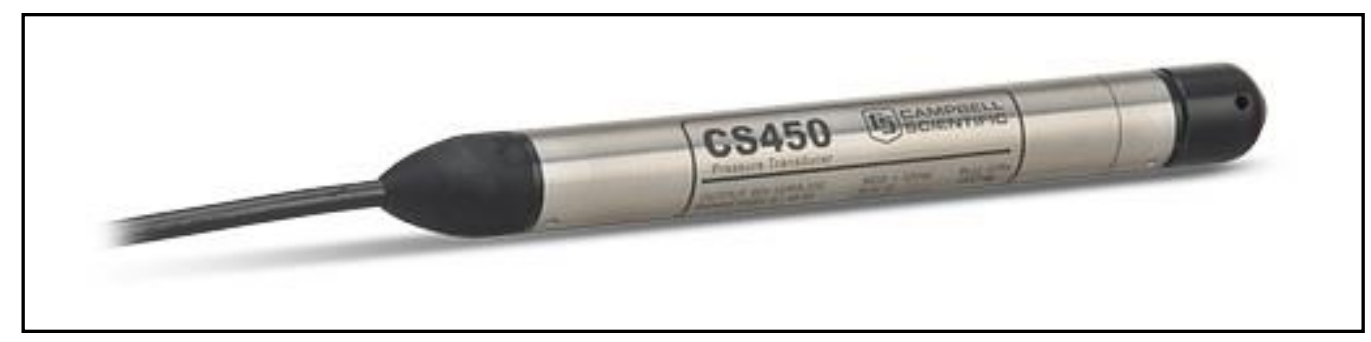

Figure 16 - Pressure Transducer, Campbell Scientific Model CS450 (image source: Campbell Scientific Co.)

The pressure transducers were placed at $2.5 \mathrm{ft}$ and then approximately at one third and two third of the length of the GI controls from the up-gradient edge, see Figure 17. The exact locations of pressure transducers match the locations of the top layer TDRs to provide ease of installation and better comparisons between collected data between by different sensors. In control 19G, the pressure transducers are installed at $2.5 \mathrm{ft}(0.7 \mathrm{~m}), 40-\mathrm{ft}(12.2$ $\mathrm{m})$, and $80-\mathrm{ft}$ (24.4 m) from the up-gradient side. Since control $19 \mathrm{H}$ is only $55-\mathrm{ft}$ long, the pressure transducers are installed at $2.5 \mathrm{ft}(0.7 \mathrm{~m}), 20 \mathrm{ft}(6.1 \mathrm{~m})$, and $40 \mathrm{ft}(12.2 \mathrm{~m})$ from the up-gradient edge. A pressure transducer was also installed in the overflow pipe in combination with a 6-inch volumetric compound weir (Thel-Mar) (model P79501 from Pollardwater $^{1}$ ) to determine the overflow volume, if any, entering the sewer system. The

\footnotetext{
1 - http://www.pollardwater.com/
} 
water level measured by the pressure transducers were used to measure the storm volume captured by the GI control and to track the changes in exfiltration performance over time.

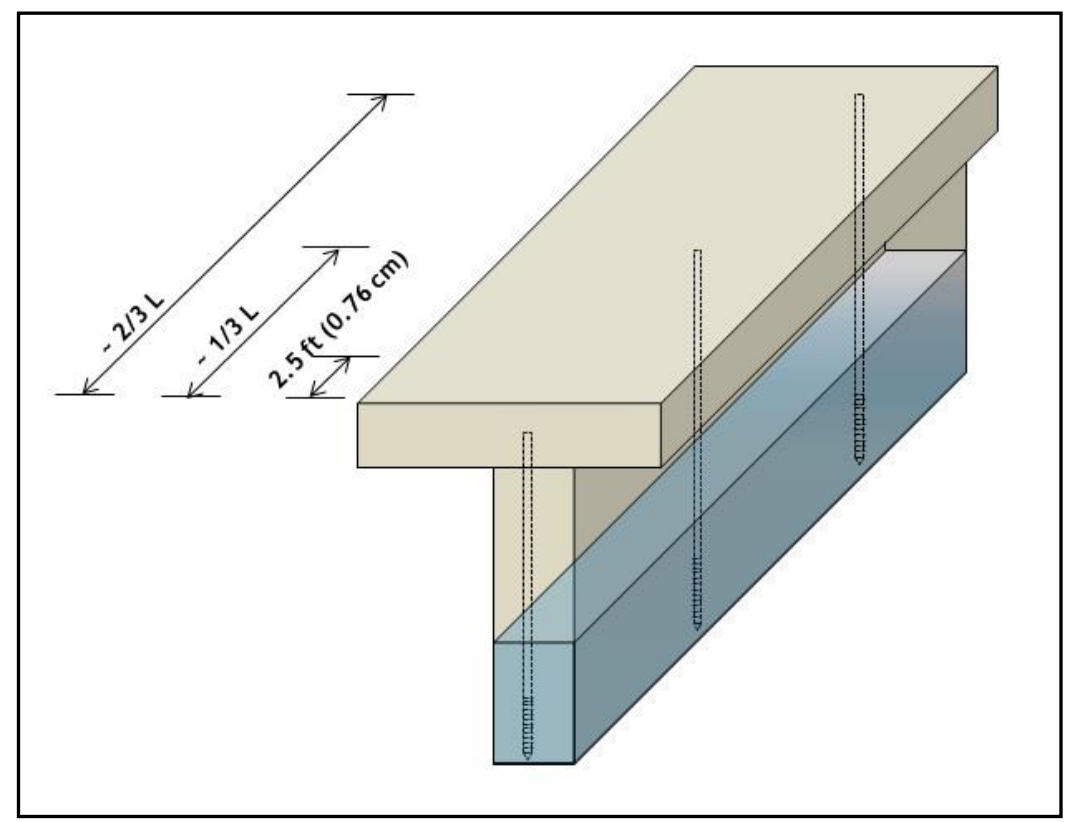

Figure 17 - Location of Pressure Transducers along the length of each GI Control

Thermistors were placed inside the surface pavers and about halfway from the surface pavers' level to the bottom of the trench, which is approximately $6.5 \mathrm{ft}(2 \mathrm{~m})$ from the bottom of the trench. The surface thermistors enable monitoring any potential freezing conditions and the urban heat island effects of permeable pavements.

The pressure transducers and TDRs also have the ability to measure temperature. The data measured by all sensors at different levels provided a temperature profile along vertical depth of the GI control. The temperature measurement also enabled monitoring the hydrological performance of the GI control while considering the effects of local changes in temperature. The variations in temperature values were expected to affect the hydrological performance, specially the exfiltration performance, through changes of the 
dynamic viscosity of water. The thermistors used are Campbell Scientific Model 108. The placement of different sensors along the depth of the GI control is shown in Figure 18, which is the cross sectional view A-A from Figure 15.

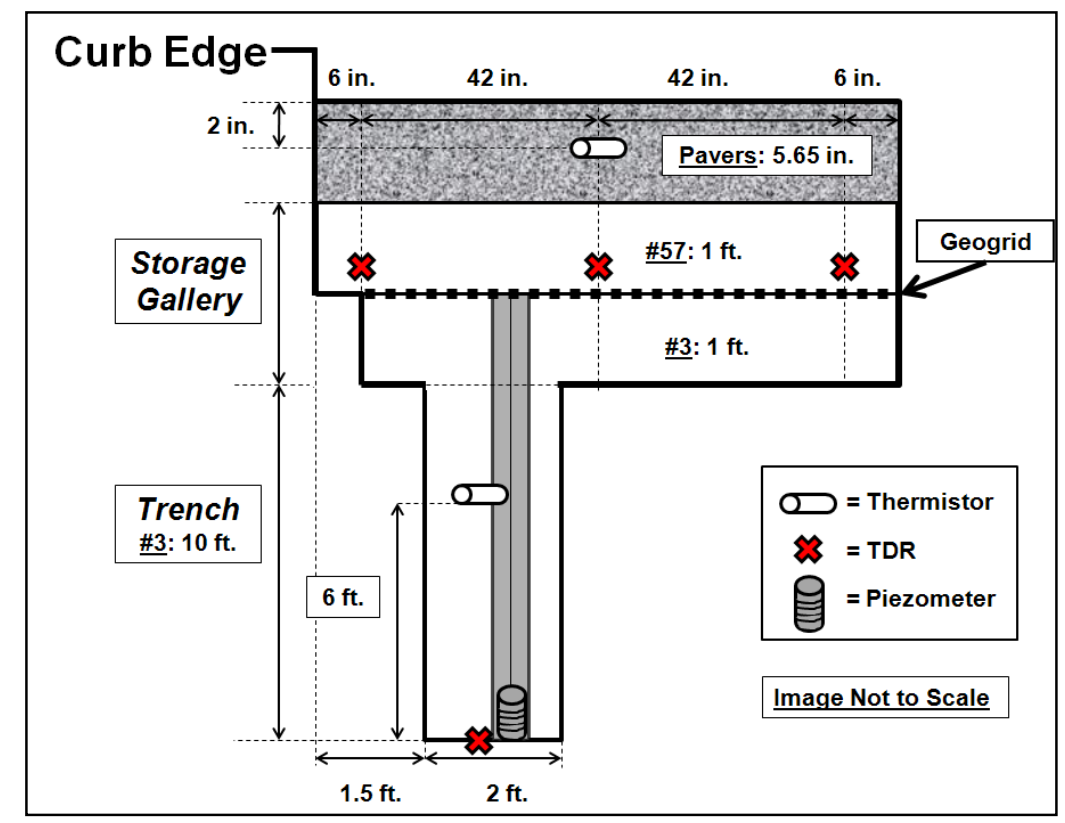

Figure 18 - Location of Different Sensors along the Depth of GI Control

\subsubsection{Instrumentation Installation}

Once the contractor excavated the storage gallery and the trench, three 15 -ft long $1^{1 / 2}$-inch diameter PVC pipes were placed at the bottom of the trench at designated positions for pressure transducers. Each pipe had a 1-ft slotted section at its bottom which was later covered with a filter sock. Pressure transducers were put inside these partially slotted PVC pipes. The TDRs and thermistors, which were supposed to be installed at the bottom of the trench and mid-depth of trench, were attached to the outside of these pipes. Because of the safety concerns installing these pipes were done from a bridge spanning the excavated area. After installing the piezometer pipes, the trench was backfilled with \#3 stone. Following installation of the geo-grid, the surface TDRs were positioned as shown 
in Figure 15. The TDRs were placed on a 1-2 inch thick layer of \#57 stone to make sure they were isolated from the geo-grid. The surface thermistors were installed at three locations along the length of the pavement strip and at approximately its mid-width. After drilling 3/8-inch diameter holes inside the surface pavers, the thermistors were inserted into the pavers and backfilled with fine glass beads and glued.

It must be noted that because of a valve box and subsequent concrete pour in control 19G, the surface TDR and piezometer cluster at 80-ft from the up-gradient edge, were moved to 75 -ft. Also in control $19 \mathrm{H}$, the TDR closest to the curb at 2.5-ft from up-gradient edge was moved from 6-in to 12-in from the curb because of a protruding concrete section coming off the sidewalk.

It must be noted that the functionality of all sensors were checked and they were calibrated prior to installation. Once the installations of the sensors were complete, all the wiring were connected to flexible conduits and eventually directed to the data logger enclosure through a set of junction boxes. Figure 19 shows different stages of instrumentation installation.

\subsubsection{Sensor Naming}

Each sensor had to be named to make the data recording possible. Since the program used by the data logger only allows a maximum length of nine character for the name of each sensor, a series of codes were used to define the GI control's location, sensor type, sensor's location within the control, and the type of data recorded by the sensor. The naming scheme is as follows: City (1 letter) - Basin Number (1 number) - Control Type (1 letter) - Data Logger Number (1 letter) - Control Associated with Respective Data logger (1 number) - Sensor Type (1 letter) - Unique Name (2 letters) - Measurement Type 
(1 letter). The unique name of each sensor describes the location of the sensor within the control. The detailed tables explaining the nine character naming scheme, unique name assignments, and measurement types are given in Appendix I. It must be noted that since the developed naming scheme is supposed to be used for the rest of GI controls in CSO130, and for other future applications of GI stormwater controls in Louisville, and even other locations across the nation by the EPA, the assigned characters and letters are more detailed than needed for monitoring controls $19 \mathrm{G}$ and $19 \mathrm{H}$ only.

\subsubsection{One-Time Measurements}

During and following installation of controls $19 \mathrm{G}$ and $19 \mathrm{H}$, a set of one-time tests were carried by UofL and the EPA, either on-site or at laboratory. These tests can be grouped into two categories: in-situ soil infiltration tests and other physical properties tests. The conducted tests and their results are explained in the following two sections.

\subsubsection{In-Situ Soil Infiltration Tests and Results}

The infiltration rates for the underlying soil layers, at the bottom of the trench and the storage gallery, were measured. Infiltration rates at the bottom of the trench were measured with two methods: 1) double ring infiltrometer (ASTM 2009; Pitt et al. 1999) and 2) Decagon minidisk tension infiltrometer (Decagon-Devices 2011). These tests were performed at three locations along the length of the storage gallery. Table 3 provides the infiltration rate values obtained from double ring infiltrometer and minidisk tests. Because

of the safety concerns, measuring the infiltration at the bottom of the trench couldn't be completed using traditional infiltration procedures such as double ring infiltrometers. 


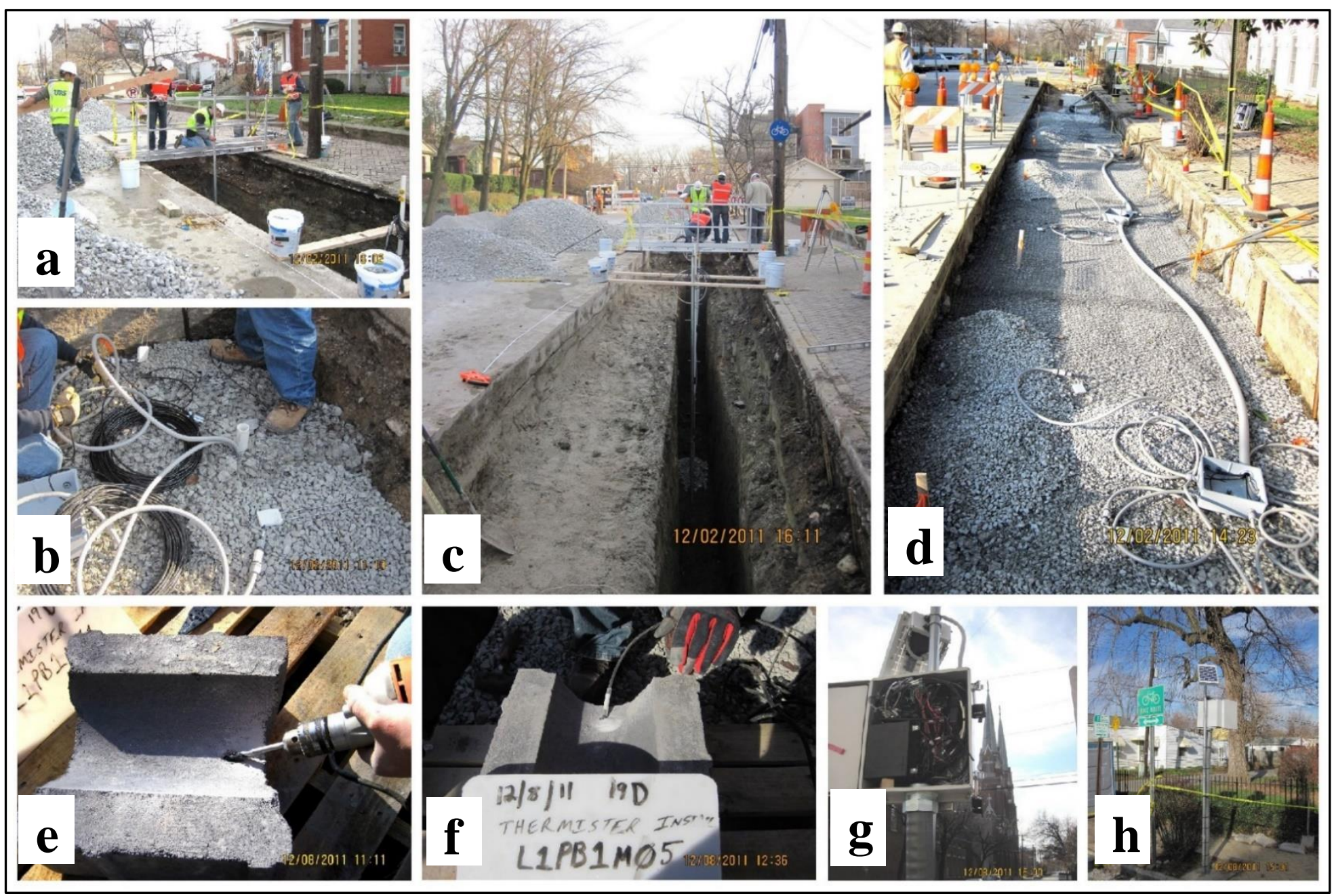

Figure 19 - Installing Sensors in Controls 19G \& 19H: a,c) installing piezometer cluster and infiltration tests at the bottom of the trench were conducted from the spanning bridge b) installing surface TDRs d) wires were passed through flexible conduits e,f) thermistor installation g,h) data logger and enclosure 
Table 3 - Measured Infiltration Rates along the Length of the Storage Gallery

\begin{tabular}{|c|c|c|c|c|c|}
\hline Control ID & & $\begin{array}{r}\text { Double } \\
\text { R }\end{array}$ & $\begin{array}{l}\text { filtration } \\
\text { hr) }\end{array}$ & Mini Dis & ation Rate \\
\hline \multirow{3}{*}{$\begin{array}{l}\text { 19G Storage } \\
\text { Gallery }\end{array}$} & Test 1 & 1.5 & \multirow{3}{*}{$\begin{array}{l}\text { Average: } \\
4.9 \pm 5.6\end{array}$} & 7.5 & \multirow{3}{*}{$\begin{array}{l}\text { Average: } \\
7.4 \pm 2.2\end{array}$} \\
\hline & Test 2 & 1.8 & & 5.2 & \\
\hline & Test 3 & 11.4 & & 9.5 & \\
\hline \multirow{3}{*}{$\begin{array}{c}\text { 19H Storage } \\
\text { Gallery }\end{array}$} & Test 1 & 18 & \multirow{3}{*}{$\begin{array}{l}\text { Average: } \\
8.9 \pm 8.5\end{array}$} & 2.1 & \multirow{3}{*}{$\begin{array}{l}\text { Average: } \\
2.6 \pm 2.6\end{array}$} \\
\hline & Test 2 & 1.3 & & 0.2 & \\
\hline & Test 3 & 7.4 & & 5.4 & \\
\hline
\end{tabular}

A falling head test method was used to estimate the infiltration rates at the bottom of the excavated trench. The method included forcing a 2 -inch schedule 40 steel pipe into the soil adjacent to the working bridge that were used for installing the piezometer cluster, by using a sledge hammer. First the water was added to the pipe to allow the underlying soil saturate for 30 minutes. Then a piezometer were placed inside the pipe and the pipe was refilled with water until the hydraulic head was equal to $10 \mathrm{ft}$ (3.05 m). Measurements were done for 90 minutes at 1-min intervals by the piezometers. The tests were repeated for three locations, close to the installed piezometer locations, along the excavated trench. The infiltration rates were calculated by estimating the slope of water level versus time and with $95 \%$ confidence interval, see Figure 20. Confidence intervals are equal to: sample estimate $\pm(\mathrm{t}$-multiplier $\times$ estimated standard error $)$, in which the $\mathrm{t}$ values are calculated with (n - 2) degrees of freedom (Montgomery et al. 2001). Table 4 summarizes the infiltration rates along the trench of controls $19 \mathrm{G}$ and $19 \mathrm{H}$.

Table 4 - Infiltration Rate Values for the Bottom of Trenches of Controls 19G and 19H

\begin{tabular}{lll}
\hline Piezometer location & $\begin{array}{l}\text { Infiltration rate }(\mathbf{c m} / \mathbf{h r}) \\
\text { control 19G }\end{array}$ & $\begin{array}{l}\text { Infiltration rate }(\mathbf{c m} / \mathbf{h r}) \\
\text { control 19H }\end{array}$ \\
\hline Upgradient & $0.119 \pm 0.033$ & $0.330 \pm 0.037$ \\
\hline Middle & $0.109 \pm 0.045$ & $0.770 \pm 0.043$ \\
\hline Downgradient & $0.015 \pm 0.043$ & $0.093 \pm 0.033$ \\
\hline Average & 0.084 & 0.398 \\
\hline
\end{tabular}



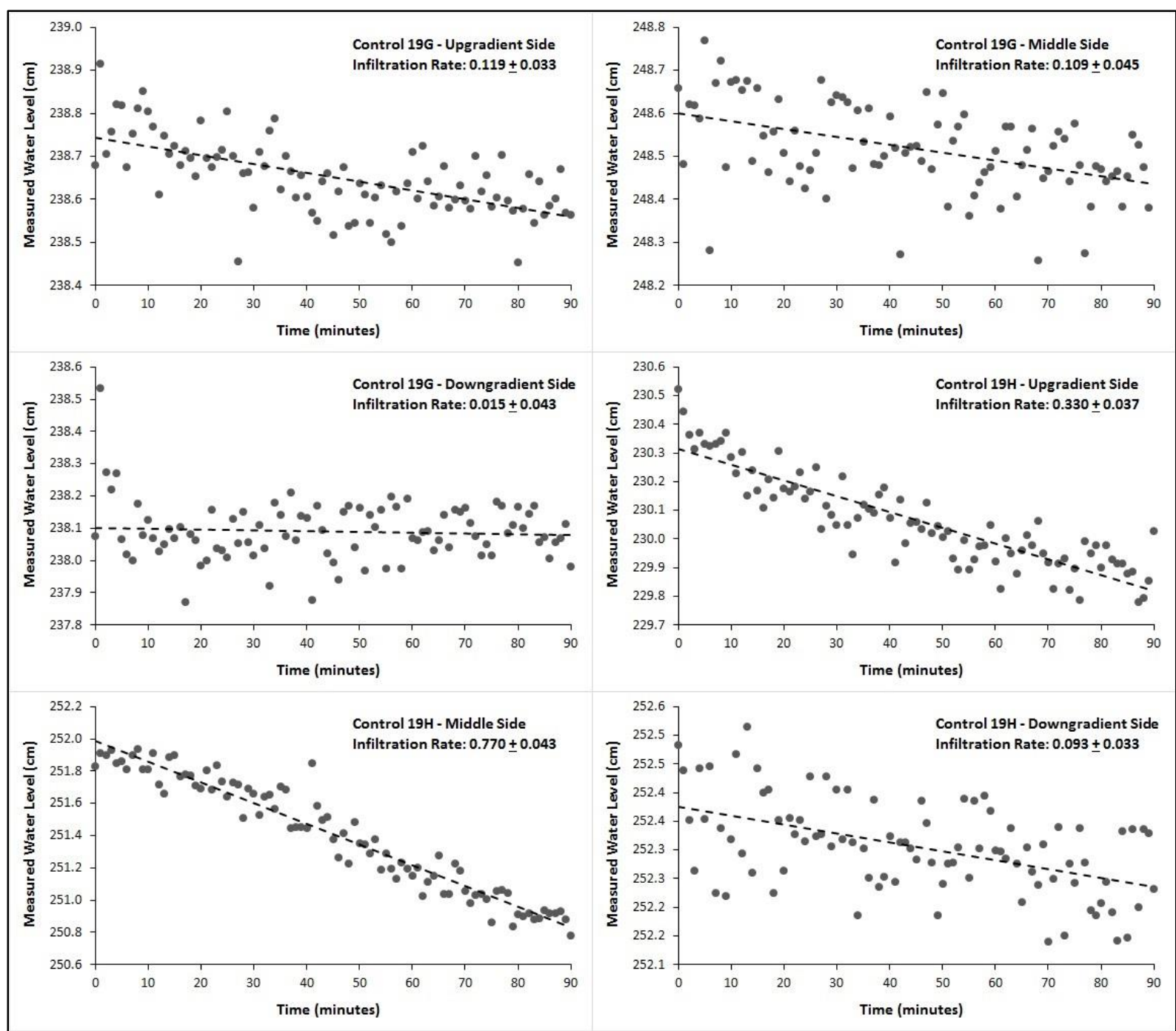

Figure 20 - Water Level versus Time for Falling Head Tests, Controls 19G and 19H

\subsubsection{Other Physical Properties Tests and Results}

The aggregates in storage layers of GI controls, AASHTO \#3 and \#57 stones, were tested for attached solids and porosity. The construction specifications required "double washed" stone for the storage layers (Montgomery et al. 2001). The maximum amount passing a $1 / 2$-inch sieve $(13 \mathrm{~mm})$ for the \#3 stone is $2 \%$ and the requirement for the \#57 aggregate is that less than $2 \%$ pass a No 8 sieve $(2.4 \mathrm{~mm})$. Samples were collected for later 
washing and determination of the mass of fines, which was done by the EPA. In this test the pre-weighed mass of collected stone samples were rinsed and scrubbed in sequential containers with appropriately sized brushes to collect the solids. The entire wash water sample were then vacuum filtered through multiple filters. The filters were oven dried to constant weight to determine the mass of sediment. Detailed sampling and test procedures, as used by the EPA, is provided in Appendix II.

Table 5 - Measured Attached Solids for \#57 Stone (data source: the EPA)

\begin{tabular}{|c|c|c|c|c|}
\hline Test & Control ID & Particle Size & $\begin{array}{l}\text { Percentage of } \\
\text { Sediment to Stone } \\
\text { (dry weight basis) }\end{array}$ & $\begin{array}{l}\text { Total Dry Percent } \\
\text { of Recovered } \\
\text { Attached Solids }\end{array}$ \\
\hline \multirow{4}{*}{$\mathbf{1}$} & \multirow{4}{*}{$19 \mathrm{G}$} & particle $>2.38 \mathrm{~mm}$ & $97.896 \%$ & \\
\hline & & $2.38 \mathrm{~mm}>$ particle $>2 \mathrm{~mm}$ & $0.030 \%$ & \multirow{3}{*}{$2.104 \%$} \\
\hline & & $2 \mathrm{~mm}>$ particle $>75 \mathrm{um}$ & $0.350 \%$ & \\
\hline & & $75 \mathrm{um}>$ particle $>1.5 \mathrm{um}$ & $1.724 \%$ & \\
\hline
\end{tabular}

Table 6 - Measured Attached Solids for \#3 Stone (data source: the EPA)

\begin{tabular}{|c|c|c|c|c|}
\hline Test & Control ID & Particle Size & $\begin{array}{l}\text { Percentage of } \\
\text { Sediment to Stone } \\
\text { (dry weight basis) }\end{array}$ & $\begin{array}{l}\text { Total Dry Percent } \\
\text { of Recovered } \\
\text { Attached Solids }\end{array}$ \\
\hline \multirow{4}{*}{1} & \multirow{4}{*}{$19 \mathrm{G}$} & particle $>12.7 \mathrm{~mm}$ & $97.210 \%$ & \\
\hline & & $12.7 \mathrm{~mm}>$ particle $>2 \mathrm{~mm}$ & $0.086 \%$ & \multirow{3}{*}{$2.790 \%$} \\
\hline & & $2 \mathrm{~mm}>$ particle $>75 \mathrm{um}$ & $1.080 \%$ & \\
\hline & & $75 \mathrm{um}>$ particle $>1.5 \mathrm{um}$ & $1.624 \%$ & \\
\hline \multirow{4}{*}{2} & \multirow{4}{*}{$19 \mathrm{G}$} & particle $>12.7 \mathrm{~mm}$ & $96.472 \%$ & \\
\hline & & $12.7 \mathrm{~mm}>$ particle $>2 \mathrm{~mm}$ & $0.685 \%$ & \multirow{3}{*}{$3.528 \%$} \\
\hline & & $2 \mathrm{~mm}>$ particle $>75 \mathrm{um}$ & $0.833 \%$ & \\
\hline & & $75 \mathrm{um}>$ particle $>1.5 \mathrm{um}$ & $2.011 \%$ & \\
\hline \multirow{4}{*}{3} & \multirow{4}{*}{$19 \mathrm{G}$} & particle $>12.7 \mathrm{~mm}$ & $97.390 \%$ & \\
\hline & & $12.7 \mathrm{~mm}>$ particle $>2 \mathrm{~mm}$ & $0.496 \%$ & \multirow{3}{*}{$2.610 \%$} \\
\hline & & $2 \mathrm{~mm}>$ particle $>75 \mathrm{um}$ & $0.435 \%$ & \\
\hline & & $75 \mathrm{um}>$ particle $>1.5 \mathrm{um}$ & $1.680 \%$ & \\
\hline
\end{tabular}


The porosity of the \#57 and \#3 stones were measured on site, on randomly selected truck loads, by UofL and the EPA, to estimate the water storage volume in the gravel storage gallery and trench. The EPA constructed two rectangular wooden frames (36-in wide by 36-in long by 5.5-in high) with a solid plywood bottom. The frames were leveled and lined with an impermeable 45-millimeter EPDM membrane. The boxes were filled with aggregate to a level above the upper edge of the EDPM. Water was added to fill the box and its volume was recorded, see Figure 21. The ratios of the water volume to the total volume of the lined frame was reported as the effective porosity. Any water absorbed by the stone were assumed negligible. Tables $7 \& 8$ summarize the results of porosity tests conducted during the construction of $19 \mathrm{G}$ and $19 \mathrm{H}$. The mean measured porosity values of the \#3 and the \#57 stones were $44 \%$ and $43.5 \%$ respectively

It must be noted that both the attached solids and porosity test procedures were time consuming and more labor intensive than anticipated during the preparation of the monitoring plan. Therefore for the second phase of construction, fewer and smaller samples were collected and both test procedures were slightly modified to save time and money.

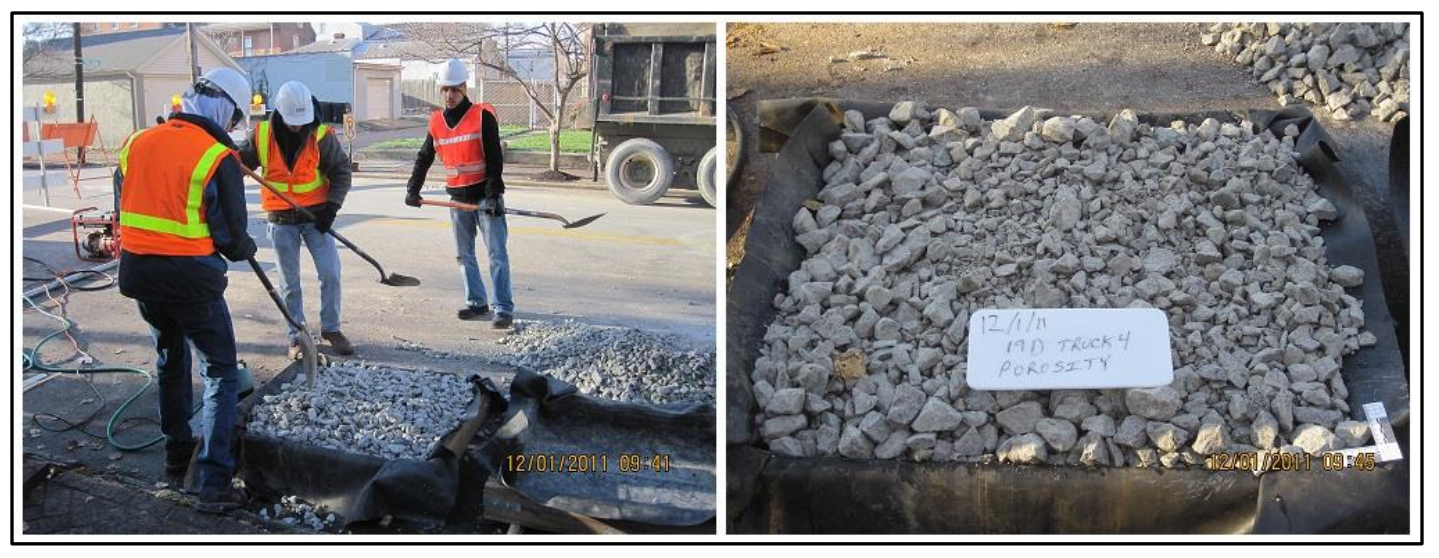

Figure 21 - Porosity Measurements during Construction of Controls 19G \& 19H 
Table 7 - Porosity Measurements Results for \#3 Stone

\begin{tabular}{ccccc}
\hline Test & Control ID & Total Volume & Water Volume & Porosity (\%) \\
\hline 1 & $19 \mathrm{G}$ & 29 & 13.5 & 46.6 \\
\hline 2 & $19 \mathrm{G}$ & 30.2 & 12.4 & 41.1 \\
\hline 3 & $19 \mathrm{G}$ & 30.3 & 13 & 42.9 \\
\hline 4 & $19 \mathrm{G}$ & 28.8 & 13.5 & 46.9 \\
\hline 5 & $19 \mathrm{G}$ & 29.8 & 12.9 & 43.3 \\
\hline 6 & $19 \mathrm{H}$ & 31 & 13.8 & 44.5 \\
\hline 7 & $19 \mathrm{H}$ & 31.2 & 12.6 & 40.4 \\
\hline 8 & $19 \mathrm{H}$ & 29.5 & 14.3 & 48.5 \\
\hline 9 & $19 \mathrm{H}$ & 30.1 & 12.6 & 41.9 \\
\hline & & & Mean Value: & $44.0 \%$ \\
\hline
\end{tabular}

Table 8 - Porosity Measurements Results for \#57 Stone

\begin{tabular}{ccccc}
\hline Test & Control ID & Total Volume & Water Volume & Porosity (\%) \\
\hline 1 & $19 \mathrm{G}$ & 28.5 & 12 & 42.1 \\
\hline 2 & $19 \mathrm{G}$ & 30.1 & 13.2 & 43.9 \\
\hline 3 & $19 \mathrm{G} / 19 \mathrm{H}$ & 30.1 & 13.7 & 45.5 \\
\hline 4 & $19 \mathrm{G} / 19 \mathrm{H}$ & 30.2 & 13.4 & 44.4 \\
\hline 5 & $19 \mathrm{H}$ & 29.9 & 12.5 & 41.8 \\
\hline & & Mean Value: & $43.5 \%$ \\
\hline & & Standard Deviation: & $1.4 \%$ \\
\hline
\end{tabular}

\subsubsection{Measurements Conducted Periodically}

\subsubsection{Surface Infiltration Testing}

UofL has been measuring the surface infiltration rates for permeable pavers, immediately after the construction and then periodically with each maintenance treatment. The infiltration tests followed a modified version of ASTM method C1701 (ASTM 2009). The method was originally developed for measuring the infiltration rates of porous concrete, but a modified version has been used by the EPA on three different permeable pavement types (porous asphalt, porous concrete, and PICP). The primary modification was to substitute the plumbers' putty with a half inch thick Neoprene sheeting, compressed 
with applied weight, to seal the 12-inch diameter PVC cylinder with the pavement surface. Additional Neoprene strips are used to seal the gaps between individual pavers' blocks to prevent water flow (Borst et al. 2010). Figure 22 shows the test apparatus while measuring the surface infiltration rates in CSO130. Four five-gallon buckets, filled with water, were used as weights to compress the Neoprene sheeting for better sealing. Detailed test procedure is described in Appendix III.

As mentioned, the surface infiltration rates were measured initially after the construction to establish the baseline infiltration capacity of the articulated pavers. The tests were repeated periodically within one week before and after each maintenance treatment. The test locations overlap with the locations of surface TDRS. The tests were initially conducted on marked locations from Figure 15: A1, A2, A4, B1, C1, C2, and C4. If any of the down-gradient locations were deemed clogged then the test location would be moved to the next TDR spot along the down-gradient direction, see Figure 15. After a year of monitoring, the results showed that the measurements along the curb are most important for evaluating the infiltration capacity of the GI control. Therefore the testing plan was revised to only conduct tests at curb edge TDRs' locations, see Table 3. All surface infiltration results are analyzed and provided in Section 6.1 of this document.

Table 9 - Surface Infiltration Testing Locations of Controls 19G \& 19H

\begin{tabular}{cccc}
\hline Control ID & Control length (ft) & $\begin{array}{l}\text { Distance from up-gradient } \\
\text { edge (ft) }\end{array}$ & $\begin{array}{l}\text { Total number of tests } \\
\text { per each control }\end{array}$ \\
\hline $19 \mathrm{G}$ & 120 & $2.5,20,40,75$ & 4 \\
\hline $19 \mathrm{H}$ & 55 & $2.5,20,40$ & 3 \\
\hline
\end{tabular}




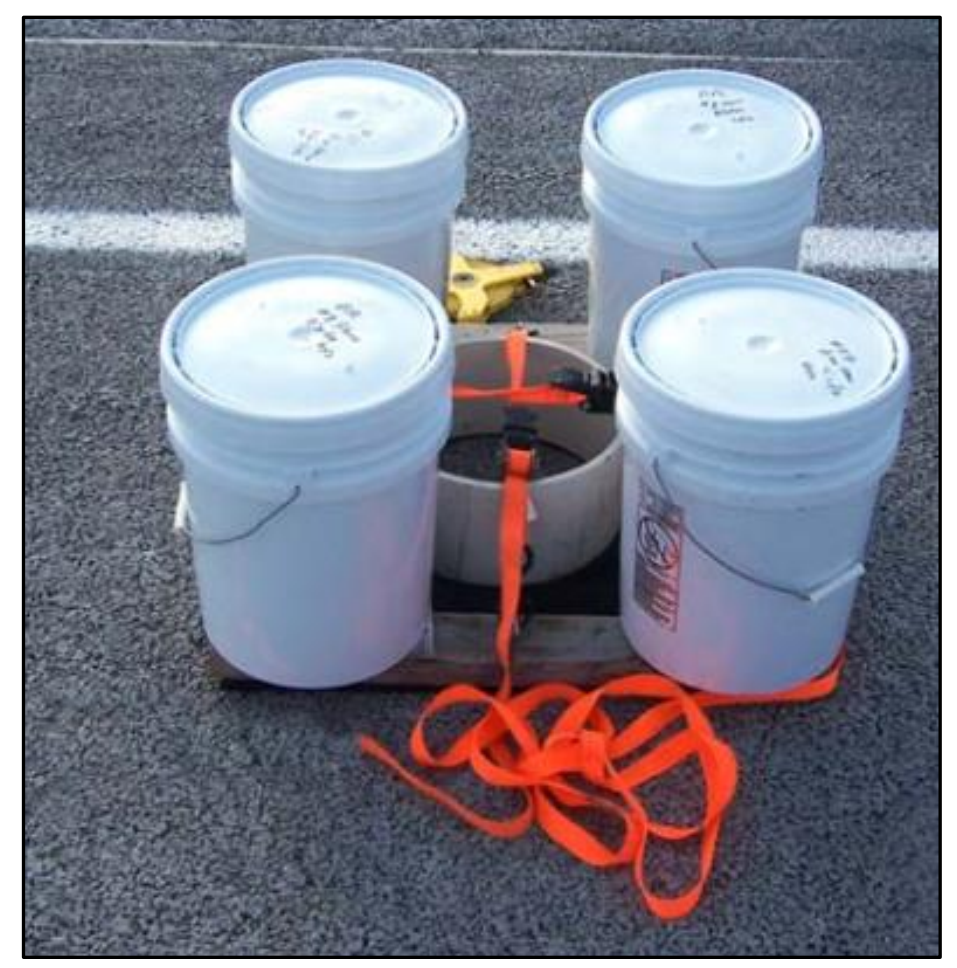

Figure 22 - Surface Infiltration Test Apparatus (Borst et al. 2010)

\subsubsection{Maintenance / Recovered Sediment Sampling}

As mentioned, UofL has been running surface infiltration tests on permeable pavers within one week before and after each surface maintenance treatments, with the exception of one treatment $(04 / 17 / 13)$ where the contractor didn't notify UofL. During the first three maintenance applications, samples were also collected from the recovered sediments and tested for particle size distribution (ASTM D6913-04) and organic matter (ASTM 297407).

Through the end of 2013, three different cleaning methods were tried on controls $19 \mathrm{G}$ and $19 \mathrm{H}$ in an effort to recover their infiltration capacity. Table 4 summarizes the treatment dates and methods tried on each control. The maintenance methods and sample analysis results are provided and discussed further in Chapter 6 of this document. 
Table 10 - Maintenance Treatment Methods Conducted on Controsl 19G and 19H

\begin{tabular}{llll}
\hline Maintenance Date & Maintenance Treatment Type & Control ID & Sediment Sampling \\
\hline $03 / 20 / 2012$ & sweeping vacuum truck & $19 \mathrm{G}$ & Yes \\
\hline $05 / 09 / 2012$ & airjet (blow out) & $19 \mathrm{G}, 19 \mathrm{H}$ & Yes \\
\hline $10 / 05 / 2012$ & airjet (blow out) & $19 \mathrm{G}, 19 \mathrm{H}$ & Yes \\
\hline $04 / 17 / 2013$ & airjet (blow out) & $19 \mathrm{G}, 19 \mathrm{H}$ & N/A \\
\hline $09 / 08 / 2013$ & Vac-head & $19 \mathrm{G}$ & N/A \\
\hline
\end{tabular}

\subsection{Data Collection}

All embedded sensors are connected to a data logger which is placed inside a water resistant enclosure. Multiple measurements recorded by sensors are stored separately in text files in comma separated value format (CSV). Each data logger is also equipped with a radio so that various data loggers ${ }^{1}$ can communicate with each other and transfer their daily collected readings to the data logger of control 19G. The data logger of control 19G is connected to a wireless modem used to transfer the collected data from all GI controls in CSO130, to FTP servers accessed by the EPA and UofL.

The rainfall data is monitored continuously by MSD's tipping bucket rain gauges installed at 19 locations over Louisville, and also through the radar data, similarly provided by the MSD. The rainfall data collected by rain gauge TR05, located near the study the area, was the main source of rainfall analysis. Since the hydrologic responses of the monitored GI controls are evaluated with respect to each rainfall event, establishing a consistent criteria for identifying rainfall events was critical. To separate events, a rainfall event was defined as greater than $0.10 \mathrm{inch}(2.54 \mathrm{~mm})$ and having an antecedent dry period of at least six hours. During the analysis of hydrological performances of the GI controls, it was observed that most rainfall events greater or equal to 0.5 inch $(12.7 \mathrm{~mm})$ induce

\footnotetext{
${ }^{1}$ Other data loggers were added to this network following the construction of the rest of GI controls in CSO130.
} 
meaningful hydrological responses from the GI controls; probably because the smaller events don't generate any significant runoff to flow into the GI controls. Therefore in this study, the events which had a cumulative depth equal or greater than 0.5 inch are used for data analysis.

\subsubsection{Data/Sampling Frequency}

All sensors including the pressure transducers, TDRs, and thermistors are programmed to collect measurements at 1-minute intervals. The collected data from each control is transferred daily to secure FTP servers through the data loggers' network as explained in the previous section. The rainfall data provided by the MSD, including raingauge TR05 and radar data are recorded at 5-minute intervals. Rain gauge data can be accessed at any time through the MSD's website ${ }^{1}$ but the radar data is only reported every six months.

The surface infiltration measurements, as previously discussed, were performed immediately after the construction and then periodically and with each maintenance treatment. Sampling from the maintenance treatments were done three times to characterize the recovered sediments.

\subsection{Conclusions}

This chapter provided a description of experimental approach taken for evaluation of hydrological performances of individual GI controls in CSO130. Various sensors' locations and conducted tests were described as well. Next chapter will explain the analysis methods used on these collected data to evaluate the hydrological performance of the GI

\footnotetext{
${ }^{1}$ Raingauge data can be accessed at: http://www.msdlouky.org/aboutmsd/rainfall_query.cfm
} 
controls $19 \mathrm{G}$ and $19 \mathrm{H}$ and the overall effectiveness of the GI controls in mitigating the CSO problem is CSO130 sewershed. 


\section{ELECTRONIC DATA ANALYSIS AND MODELING}

This chapter reviews analysis methods and their results on electronically collected data from the embedded sensors in controls $19 \mathrm{G}$ and $19 \mathrm{H}$. Installation of these sensors were finished on December $12^{\text {th }} 2011$ and the electronic data analysis covers a period from mid-December 2011 through the end of year 2013.

\subsection{Rainfall Data}

Analyzing the rainfall data is a key component in evaluating the hydrological performance of GI controls, since the electronic data recorded by the embedded sensors should be interpreted with respect to rainfall events. As mentioned previously, the rainfall data within the CSO130 sewershed is estimated by using the MSD's nearest rain gauge (rain gauges TR05) and through the Radar data ${ }^{1}$. The study area is approximately located at the center of three radar pixels so to get an estimate of the rainfall depth, the three rain

depth values from each pixel are averaged. Each radar pixel has approximately an area of 1 square kilometer.

\footnotetext{
${ }^{1}$ Rader data is provided through NEXRAD (Next-Generation Radar) which is a network of weather radar operated by National Weather Service (NWS).
} 
Since the radar data is only made available to MSD and UofL every six months, the rain gauge TR05's data is used as the main source for rainfall analysis. After comparing the events identified from TR05 data with the radar data and responses from embedded sensors in controls $19 \mathrm{G}$ and $19 \mathrm{H}$, a few events which were missed by raingauge data were identified and added to the original data.

For this study, a rainfall event was defined as any rain event with greater than 0.10 inch (2.54 $\mathrm{mm})$ accumulation and having an antecedent dry period of at least six hours. It was observed that primarily events greater than or equal to 0.5 inch $(12.7 \mathrm{~mm})$ induce significant hydrological responses from the GI controls and were thus used for most data analyses. To achieve MSD's overflow reduction goal at CSO130 (provided in Table 1), rainfall data for year 2001 (typical design year) is used. Figure 23 and Table 11 provide a comparison between the rainfall events for years 2001, 2012, and 2013. As it can be seen the rainfall depths and distributions, the rain events recorded during the 2012-2013 study period were reasonably similar to the 2001 design event year. While the close match between the design event year and the 2012-2013 study year was unexpected, this provides a relatively straight forward comparison of the individual hydrological responses observed during the study year to their intended goals. This is satisfactory since the individual hydrological responses and effectiveness of GI controls can be compared to their intended design goals. 


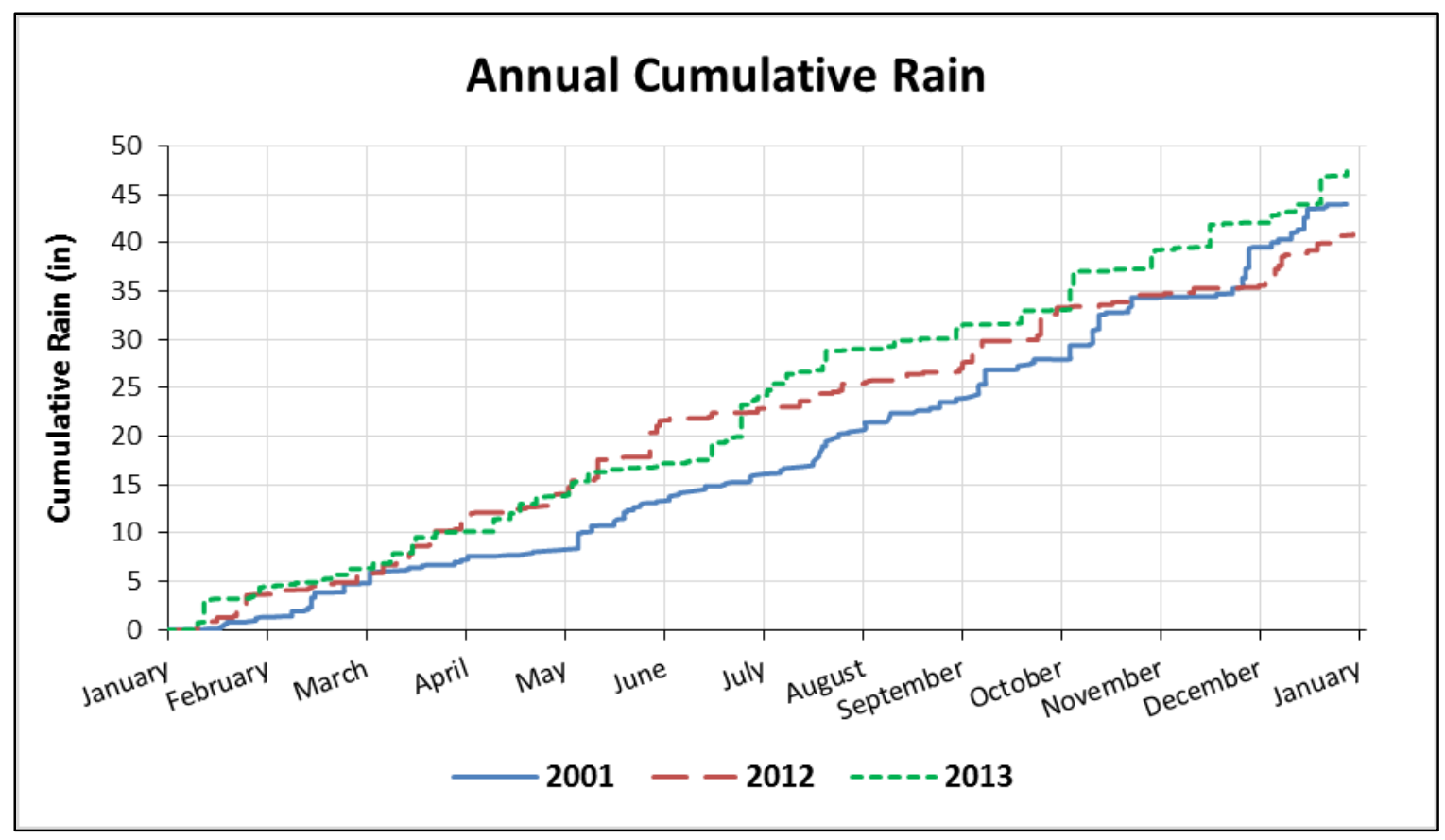

Figure 23 - Comparison of Annual Cumulative Rain for Years 2012, 2013 (Study Period) and 2001 (Design Year)

Table 11 - Comparison of Rainfall Events for Years 2012, 2013 (Study Period) and 2001 (Design Year)

\begin{tabular}{llll}
\hline Year & Number of Events & $\begin{array}{l}\text { Number of Events } \\
\text { Greater than } \mathbf{0 . 5} \text { inch }\end{array}$ & $\begin{array}{l}\text { Total Annual Cumulative } \\
\text { Rain Events (inches) }\end{array}$ \\
\hline 2001 & 68 & 33 & 44.00 \\
\hline 2012 & 71 & 28 & 40.88 \\
\hline 2013 & 65 & 30 & 47.3 \\
\hline
\end{tabular}

Figure 24 shows the monthly rainfall depths for years 2001, 2012 and 213. As it can be seen on the graph, except for the months of February, August, and November average monthly rainfalls for years 2012 and 2013 have been roughly uniform through the study period which provides satisfactory and sufficient data for monitoring seasonal changes of hydrological responses from controls $19 \mathrm{G}$ and $19 \mathrm{H}$. 


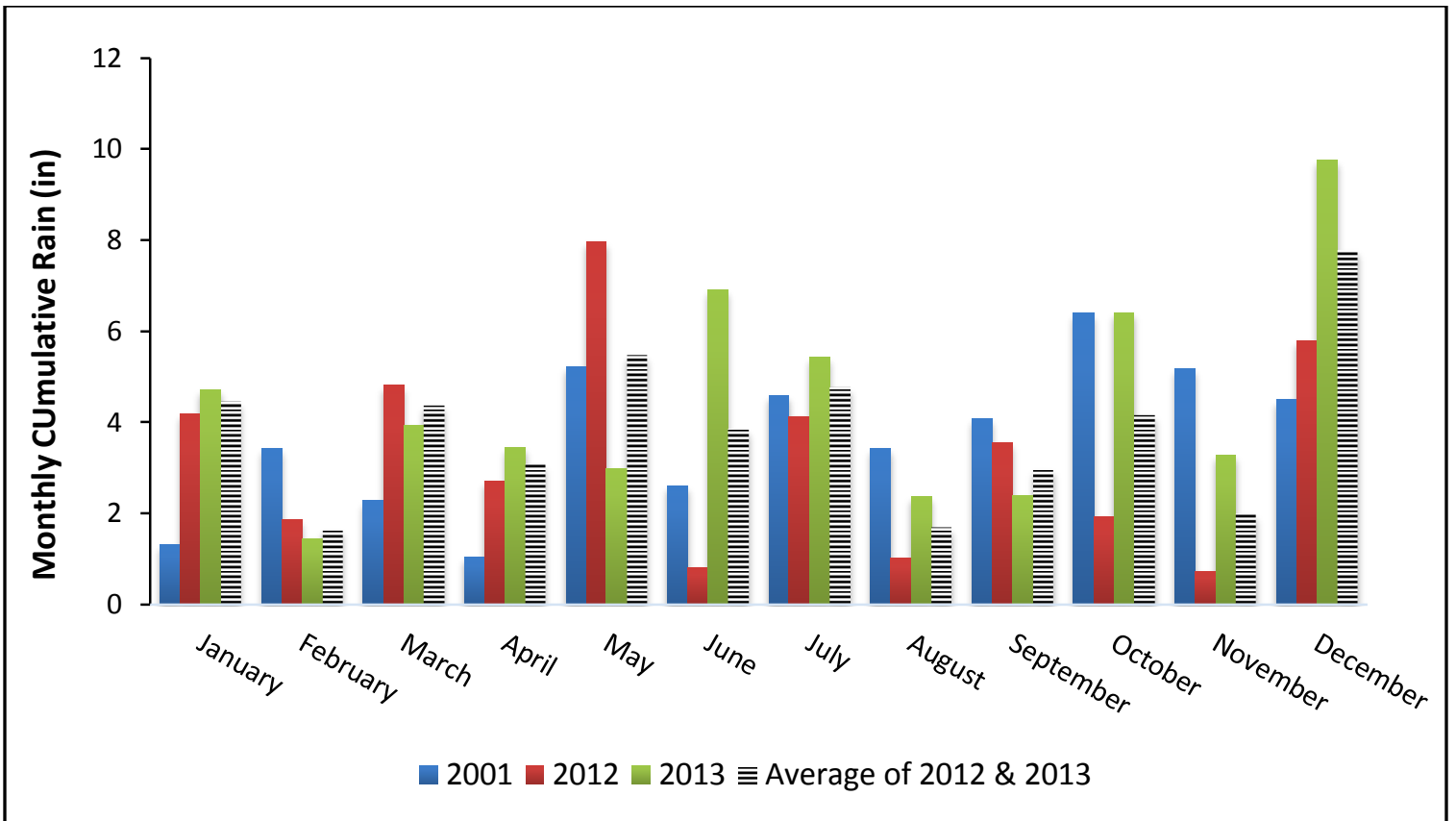

Figure 24 - Monthy Rainfall Depths for Years 2012, 2013 (Study Period) and 2001 (Design Year)

\subsubsection{Rainfall Intensities}

Rainfall intensity, which quantifies the rate of falling rain, varies with time during a rainfall event so it is usually calculated as an average value for segments of storm period. As previously mentioned in Section 3.4.1 of this document, all rainfall measurements are recorded at 5-minute intervals which means any measurable rainfall is reported for the previous 5 minutes, therefore the rainfall intensities in this research are calculated for 5minute and 15-mintue segments. An average intensity is also calculated by averaging all 5-min intensity values during the storm period. Eventually by dividing the measured rain depth for the rainfall event by the duration of rainfall an average intensity of rainfall events is calculated. All intensity values are expressed in inches per hour. Figure 25 shows the maximum 5-minute intensity values versus the 15-min intensity values for the study period. 
As it can be seen, the two parameters have a direct relationship with each other but the 15min intensity values are normally smaller than 5-min intensity values.

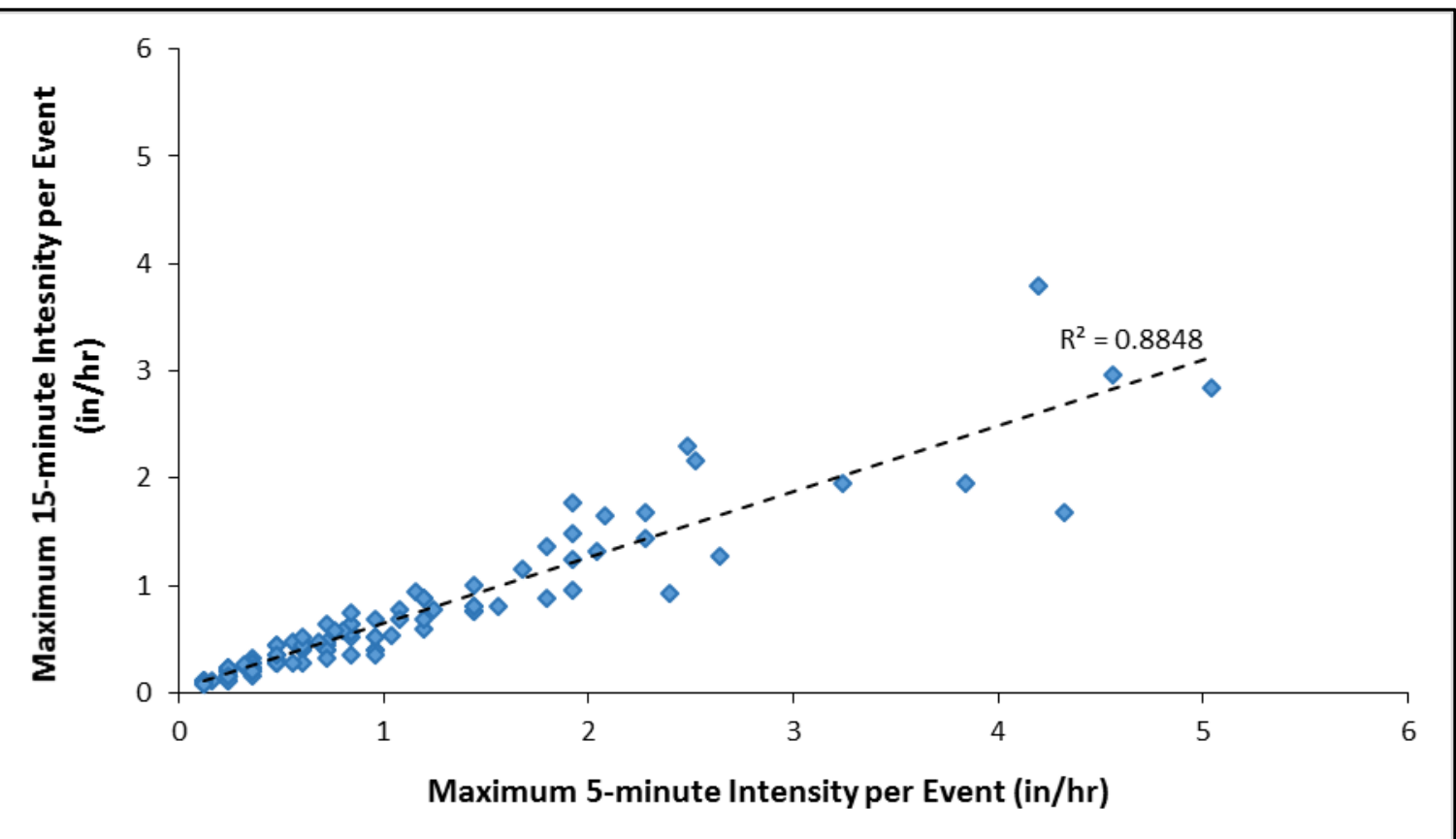

Figure 25 - Maximum 5-minute Intensity Values Versus Maximum 15-minute Intesity Values

\subsection{Pressure Transducer Data}

Six pressure transducers were installed along the length and inside the trenches of controls $19 \mathrm{G}$ and $19 \mathrm{H}$ (three in each GI control) which enable measuring the captured stormwater level inside the storage layers (\#3 and \#57 stones) of these two controls. The data collected from these sensors can be utilized for evaluating the exfiltration rates and total volume captured by each GI control per each storm event. It was observed that during rainfall events, as stormwater runoff infiltrates into the GI control the water level rises very quickly and then gradually drops as the captured volume exfiltrates into the surrounding and underlying soil layers. Figures 26 and 27 show the changes in measured water level inside controls $19 \mathrm{G}$ and $19 \mathrm{H}$ for the first few weeks after their installation. The secondary 
horizontal axis (on top) shows the cumulative rain depth per each rainfall event. Table 12 shows the exact location of pressure transducers along length of each control, measured from the upgradient edge.

Table 12 - Locations of the Three Pressure Transducers along the Length of each GI Control

\begin{tabular}{lllll}
\hline Control ID & Control's Length & Location 1 & Location 2 & Location 3 \\
\hline $19 \mathrm{G}$ & $120 \mathrm{ft}$ & $2.5 \mathrm{ft}$ & $40 \mathrm{ft}$ & $75 \mathrm{ft}$ \\
\hline $19 \mathrm{H}$ & $55 \mathrm{ft}$ & $4.5 \mathrm{ft}$ & $20 \mathrm{ft}$ & $39 \mathrm{ft}$ \\
\hline
\end{tabular}

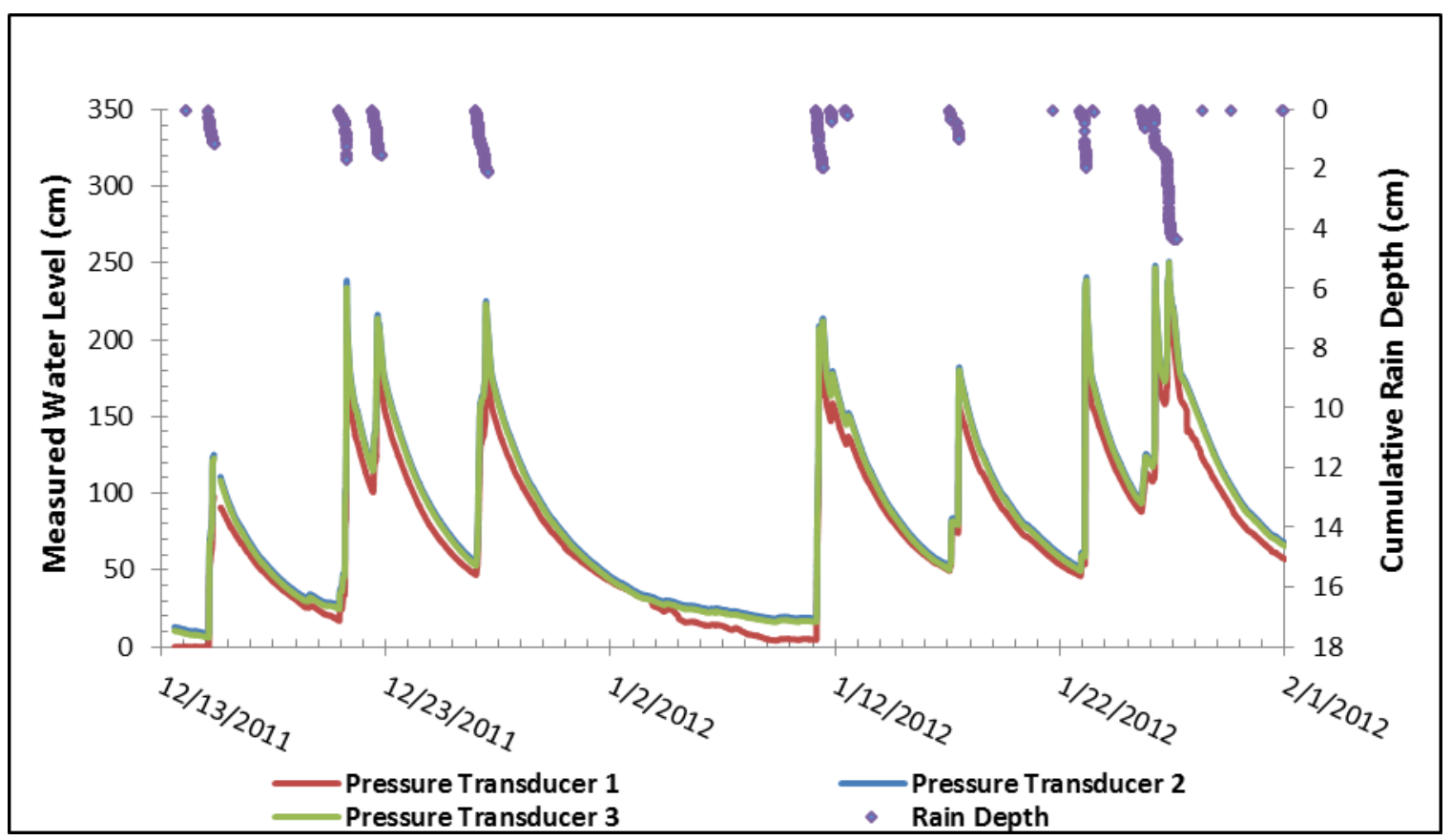

Figure 26 - Changes in Captured Water Level, Control 19G

When comparing Figures 26 and 27, it is noted that the captured water level in control 19G, contrary to control $19 \mathrm{H}$, never reaches zero values. This is not unexpected since the infiltration tests conducted during construction showed higher infiltration rates for control 19H's underlying soil layers compared to control 19G's, see Table 4. Figures 
showing all water level changes in both controls (for the study period), are included in Appendix IV.

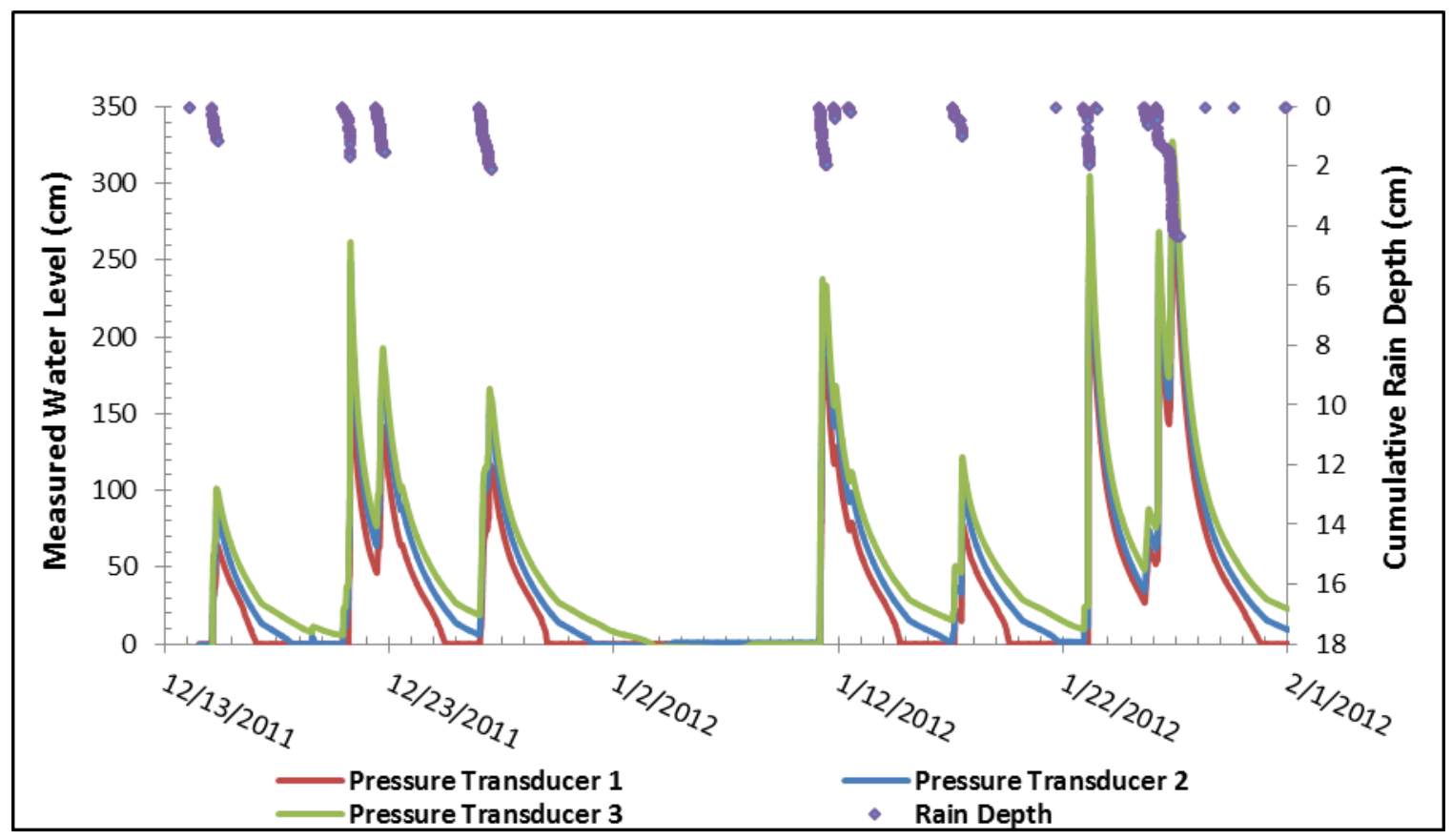

Figure 27 - Changes in Captured Water Level, Control 19H

It was expected that measured water level values from different pressure transducers would have a constant offset from each other due to the existing slope at the bottom of the trenches. While such a relationship can be identified in the recorded values in the second and third pressure transducers, the first pressure transducer exhibited a delayed and relatively irregular response as compared to the other two pressure transducers, see Figure 28. The irregular response is suspected to be the result of sediment accumulation along the slotted section of both most upgradient pressure transducer' pipe in each control. It is speculated that the sediments 1) came off from the soil layers along the upgradient wall of each trench, 2) accumulated when the remaining construction debris were washed into the GI controls with the first rainfall events, or 3) a combination of both. To investigate 
this hypothesis a laboratory study was conducted which modeled the sediment accumulation around the slotted section of a pressure transducer pipe and the results confirmed the hypothesis. Because of this observed incident the water levels only measured from the second and third pressure transducers are used in all calculations and modeling efforts of this study.

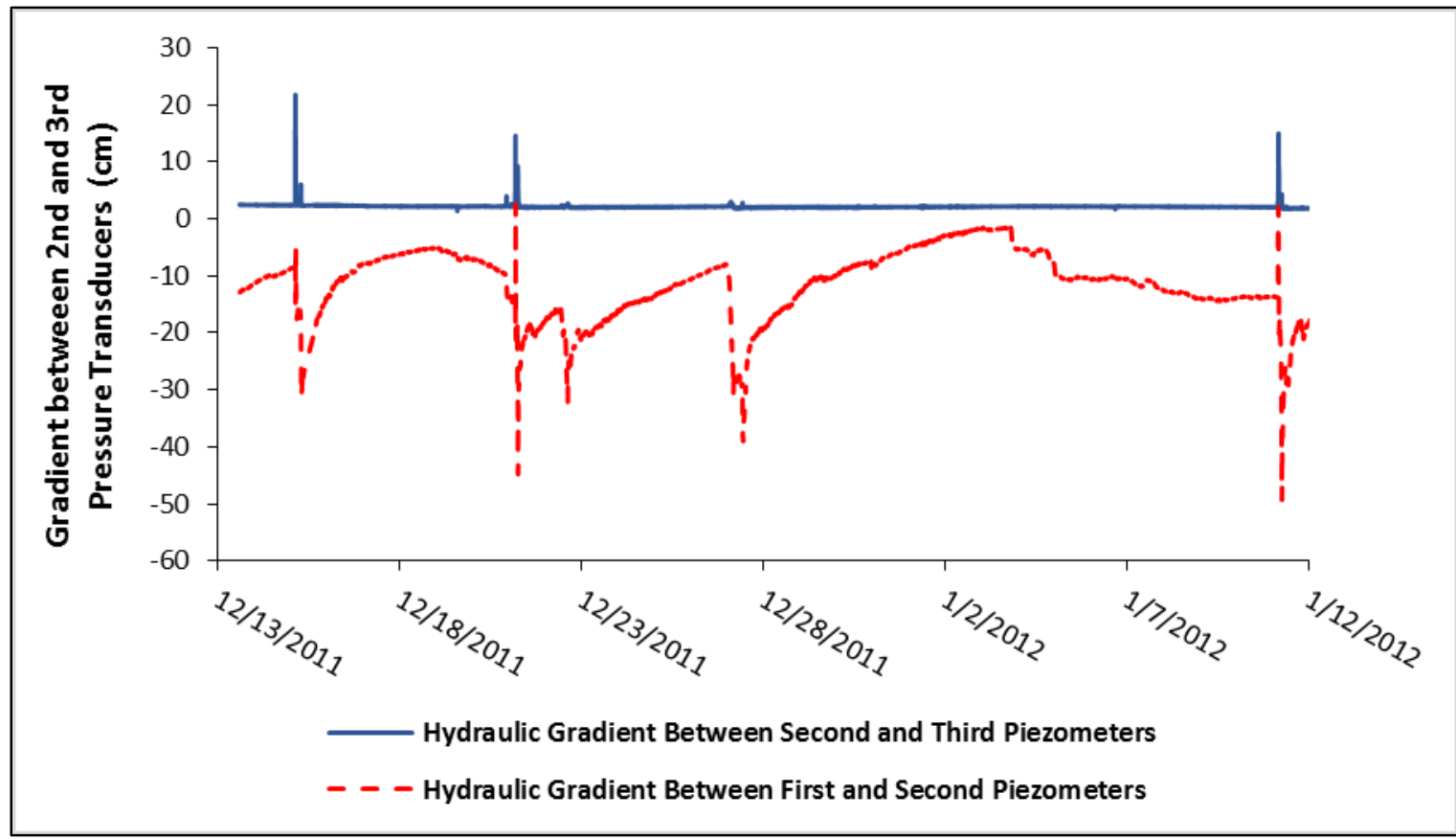

Figure 28 - Differences between Measured Water Levels from the three Pressure Transducers, Control 19G

The current hypothesis is that stormwater runoff flows along the curb edge until it reaches the GI controls. The stormwater then infiltrates into the control through the first available pavement openings. During intense rainfall events, the infiltrated stormwater accumulates at the base of the gallery faster than it spreads along the length of the control and, as a result, a temporary hydraulic gradient develops between the measured water levels from different pressure transducers. As the clogging advances the infiltration point into the 
gallery moves along its length toward the downgradient edge until eventually the gradient reverses, see Figure 29. This is more evident in control 19G since it is longer than control $19 \mathrm{H}$ and more spacing is available between pressure transducers.

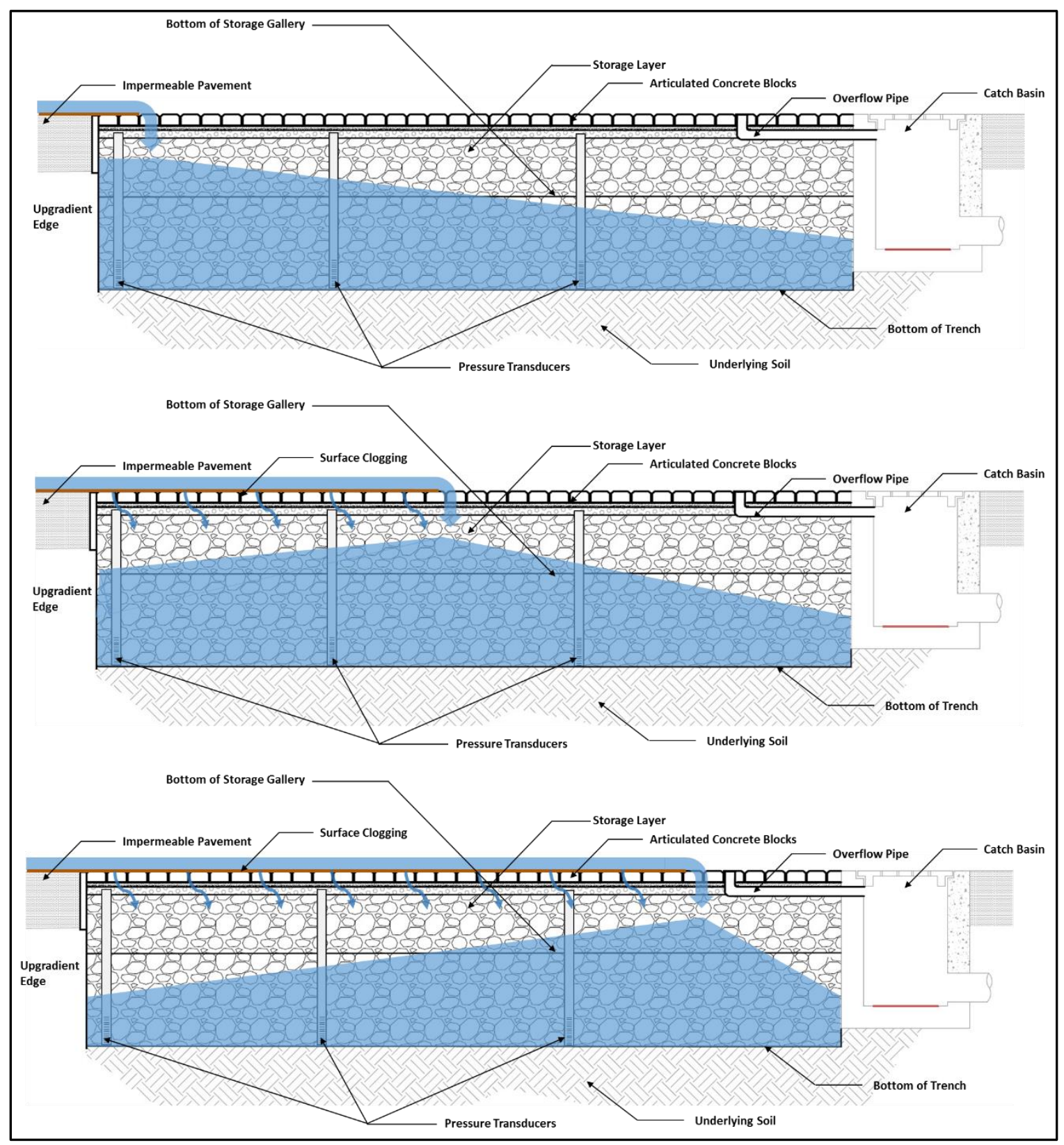

Figure 29 - Developed Gradient in the GI Control as Surface Clogging Advances 
Figures 30 and 31 show the hydraulic gradient developed between the second and third pressure transducers in control $19 \mathrm{G}$ a few months after its installation and during a successful maintenance in 2013, respectively. The values recorded by these two pressure transducers have a constant offset from each other during the dry weather conditions equal to 2.5 centimeters. In each figure the secondary horizontal axis shows the 5-min intensity values per rainfall events. Once clogging reaches the downgradient side the developed hydraulic gradient is less evident but with each successful maintenance treatment the gradient development returns to its original pattern. One of the successful maintenance treatments for control $19 \mathrm{G}$ is marked with a vertical green line in Figure 31. Different surface maintenance treatment types and their effectiveness are discussed in more detail in Chapter 6.

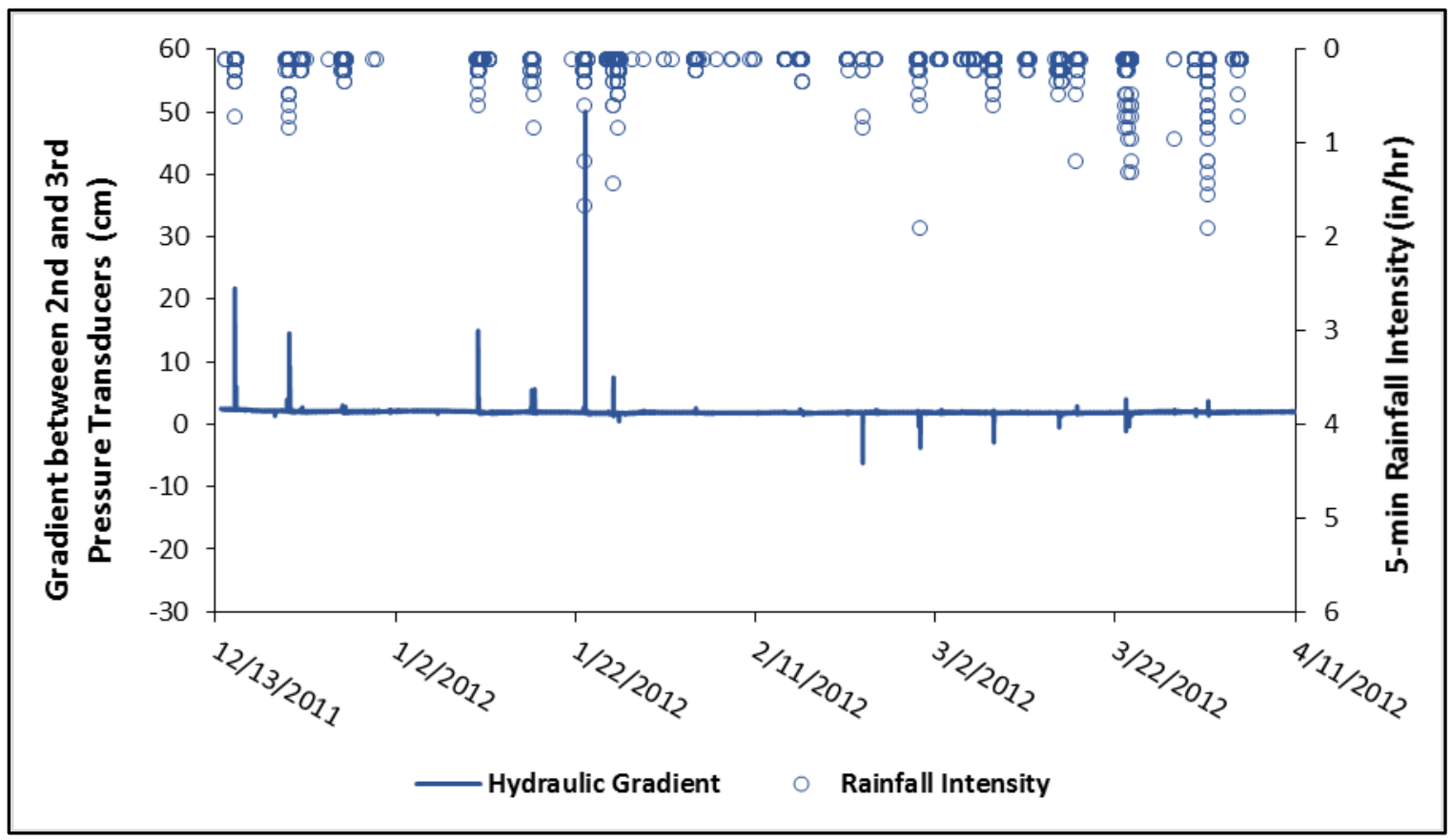

Figure 30 - Developed Gradient in Control 19G Following its Construction 


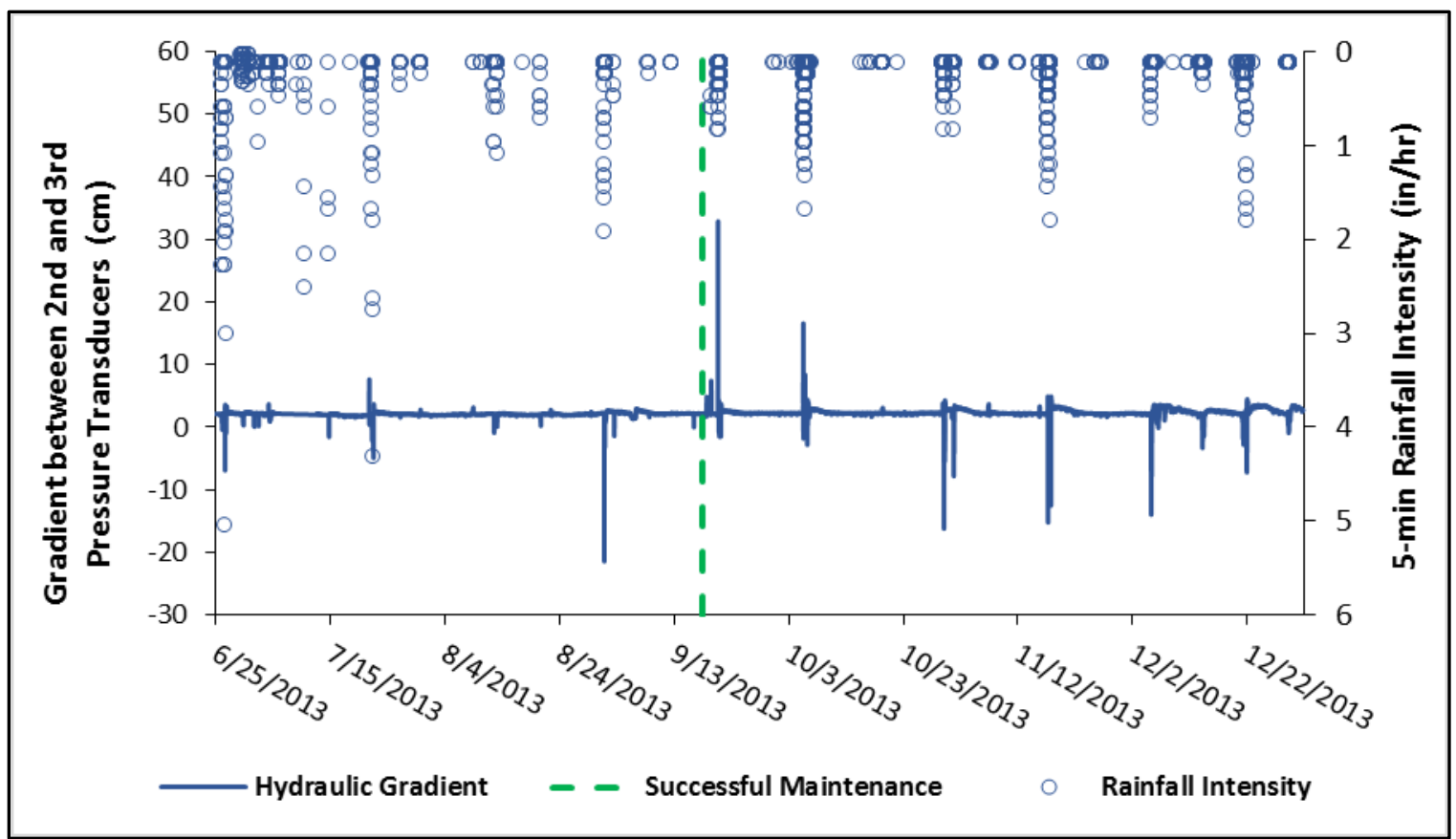

Figure 31 - Developed Gradient in Control 19G before and after a Successful Maintenance

During the study period the water level in control 19G never reached higher than the trench level and never filled the storage gallery. In contrast, the water level in control 19H filled the trench and reached the storage gallery 5 times in 2012. One of these events in Control $19 \mathrm{H}$ was significant enough to also fill the storage gallery and overflow into the combined sewer system. This rainfall event that happened on 5/29/2012 was 2.51 inches and lasted about 6 hours. Graphs showing changes in measured water levels during the study period, for each GI control, are included in Appendix IV.

\subsubsection{Exfiltration Rates}

As mentioned earlier, with each rainfall event the water level inside the control quickly rises as the water infiltrates into the control and then gradually drops as the captured water exfiltrates into the underlying and surrounding soil layers. These rise and 
drop of captured stormwater represents the infiltration and exfiltration performances respectively.

To quantify the changes in exfiltration performance, recession (drawdown) and exfiltration rates are calculated during each water level drop following a rainfall event. Recession rates are the rate at which the water level drops over time. Figures 32 and 33 show the recession rates for the first five rainfall events following the installation of Controls $19 \mathrm{G}$ and $19 \mathrm{H}$. As shown on these figures the recession rates decrease with a decrease of water level which is expected because of the decrease in hydraulic head and subsequently the exfiltration area. An empirical equation has been estimated for recession rates versus changes of the water level for control $19 \mathrm{H}$. A recession rate versus water level equation could not be developed for control 19G due to the sudden decrease of rates for levels smaller than $200 \mathrm{~cm}$. This is suspected to be because of presence of sand layers, with higher hydraulic conductivity values, in the upper levels of the trench of Control 19G, see Figure 32. 


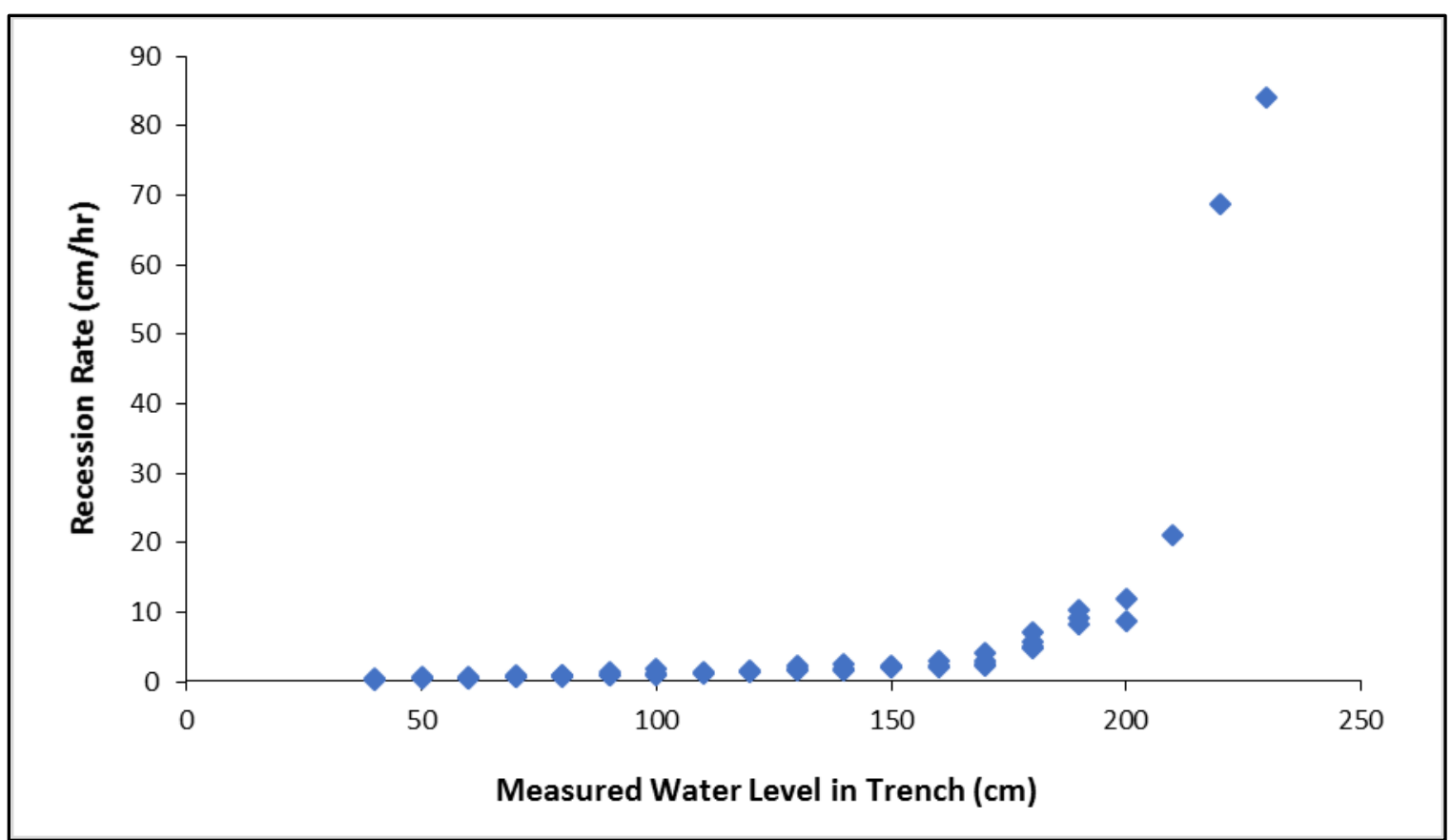

Figure 32 - Changes of Recession Rates versus the Water Depth, Control 19G

To better compare the initial exfiltration performances of controls $19 \mathrm{G}$ and $19 \mathrm{H}$, average recession rates are calculated for the first five rainfall events and are shown in logarithmic scale in Figure 34. For water levels less than $170 \mathrm{~cm}$ the trend of changes in recession rates for control $19 \mathrm{G}$ follows the same pattern as control $19 \mathrm{H}$, but with slower average recession rates. The slower recession rates in $19 \mathrm{G}$ are expected due to the lower permeability materials measured at the bottom of $19 \mathrm{G}$ as compared to $19 \mathrm{H}$, see Table 4 . 


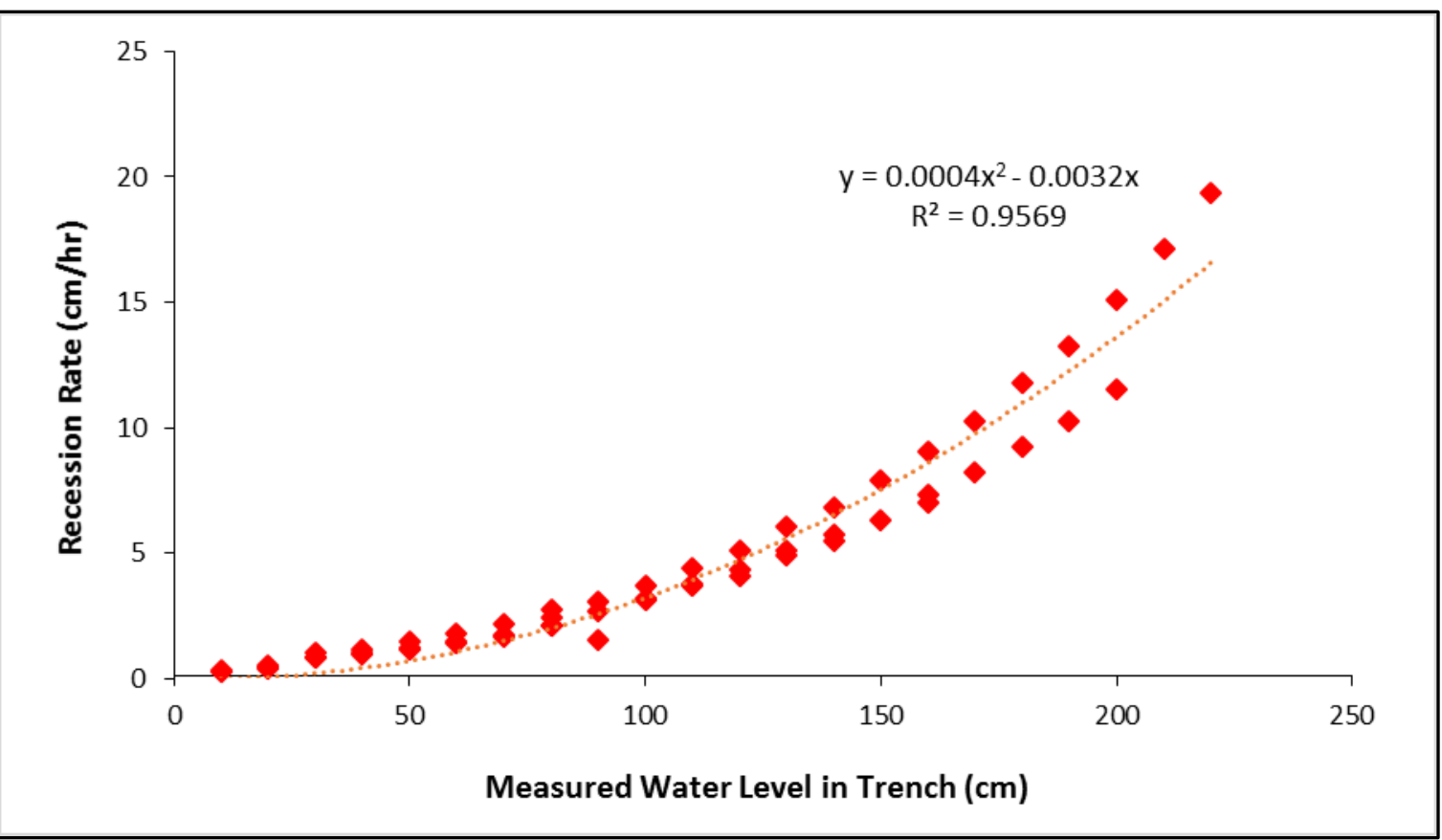

Figure 33 - Changes of Recession Rates versus the Water Depth, Control 19H

The exfiltration rates for both controls are calculated similarly to recession rates, for $10-\mathrm{cm}$ intervals of water level measured from the bottom of the trench. Unlike the recession rates, however, the exfiltration rates are calculated based on the volume of exfiltrated stormwater through the available exfiltration area, which is equal to total wetted area. The following formula provides the equation by which the exfiltration rates are calculated with for 10 -cm intervals of water level:

$$
E R_{z}=\frac{\frac{\text { Volume of Exfiltrated Storm Water }}{\text { Exfiltration time }}}{\text { Wetted Surface Area }}=\frac{\frac{n \times 10 \mathrm{~cm} \times x y}{t_{z-5 \mathrm{~cm}-t_{z+5 \mathrm{~cm}}}}}{x y+2 z x+2 z y}
$$

In which,

$\mathbf{E R}_{\mathbf{z}}$ : exfiltration rate for the water level $z$

$\mathbf{n}$ : the porosity of the storage layer equal to 0.4 , which is intentionally underestimated to account for post-construction settlement and compaction of 
stone layers, based on the porosity test results conducted during construction, see Tables 7 and 8.

$\mathbf{x}$ : width of the trench, equal to $2 \mathrm{ft}(61 \mathrm{~cm})$ for both controls $19 \mathrm{G}$ and $19 \mathrm{H}$

y: length of the trench, equal to $120 \mathrm{ft}(3660 \mathrm{~cm})$ for $19 \mathrm{G}$ and $55 \mathrm{ft}(1677.5 \mathrm{~cm})$ for control $19 \mathrm{H}$

z: captured stormwater depth measured from the bottom of the trench

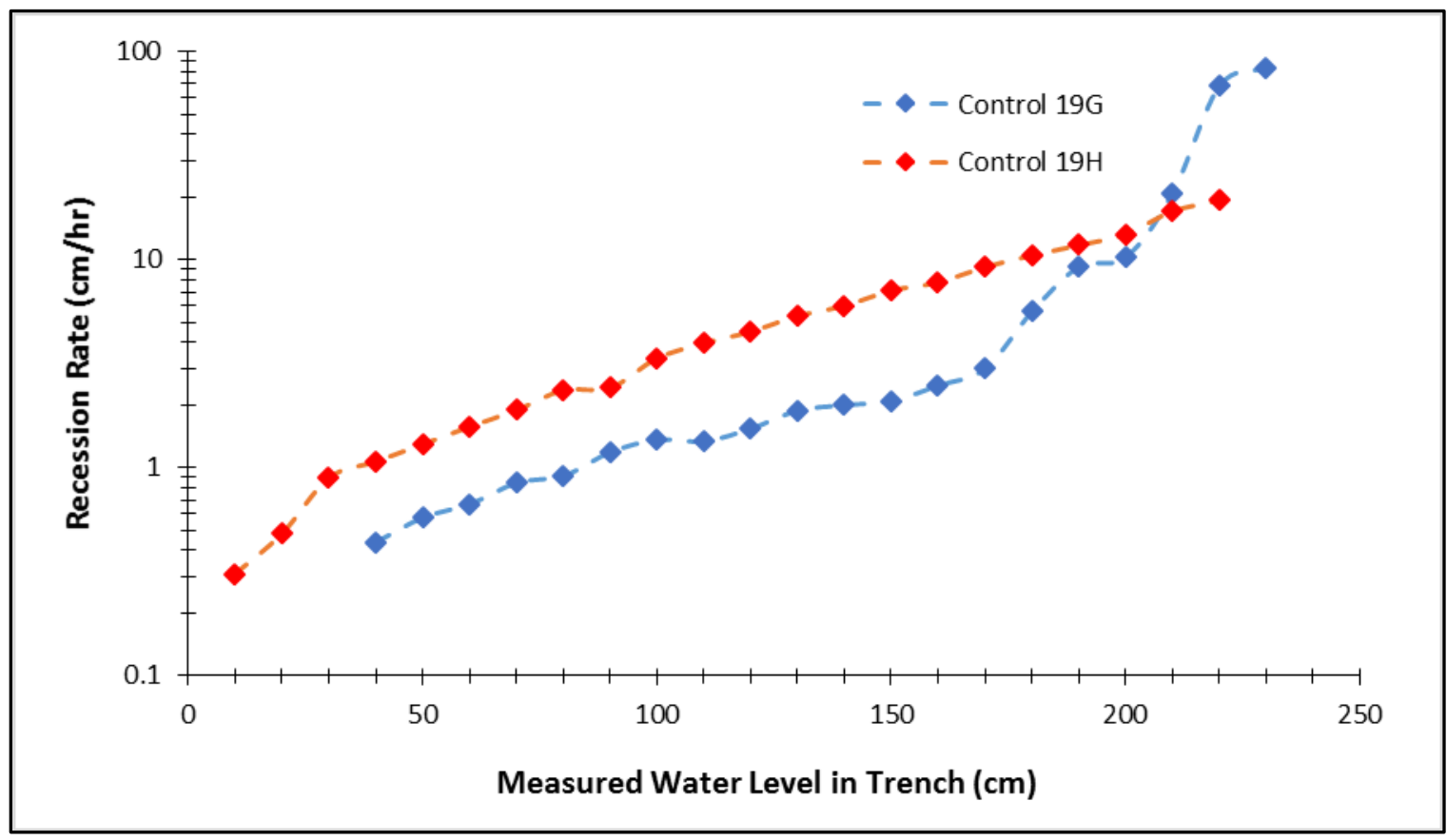

Figure 34 - Mean Recession Rates for Controls 19G and 19H for the first Five Rainfall Events

A total number of 58 rainfall events were selected for estimating the exfiltration rates during the study period. Table 13 summarizes the water levels and the number of rainfall events that the exfiltration rates are calculated and analyzed for.

Figure 35 shows the average exfiltration rates for the first five rainfall events for controls 19G and 19H. Similar to Figure 34, the Y-axis (exfiltration rates) in Figure 35 is shown in logarithmic scale to provide better comparison between the two controls. As expected the exfiltration rates for control 19G are slower than control 19H for water levels 
less than $200 \mathrm{~cm}$. Figure 36 shows that a third order polynomial equation can estimate the exfiltration rates based on the measured water level for control $19 \mathrm{H}$ and also for water levels less than $170 \mathrm{~cm}$ in control 19G.

Table 13 - Water Levels and Number of Rainfall Events Analyzed for Estimating the Exfiltration Rates, Controls $19 \mathrm{G}$ and $19 \mathrm{H}$

\begin{tabular}{llll}
\hline $\begin{array}{l}\text { Water Depth } \\
(\mathrm{cm})\end{array}$ & $\begin{array}{c}\text { Control 19G } \\
\text { Number of Rainfall } \\
\text { Events }\end{array}$ & $\begin{array}{l}\text { Water Depth } \\
(\mathrm{cm})\end{array}$ & $\begin{array}{l}\text { Control 19H } \\
\text { Number of Rainfall } \\
\text { Events }\end{array}$ \\
\hline 60 & 17 & 20 & 26 \\
\hline 80 & 24 & 40 & 38 \\
\hline 100 & 38 & 60 & 45 \\
\hline 110 & 39 & 80 & 41 \\
\hline 120 & 38 & 100 & 33 \\
\hline 140 & 35 & 120 & 24 \\
\hline 160 & 31 & 140 & 16 \\
\hline 180 & 22 & 160 & 13 \\
\hline 200 & 11 & 180 & 11 \\
\hline
\end{tabular}




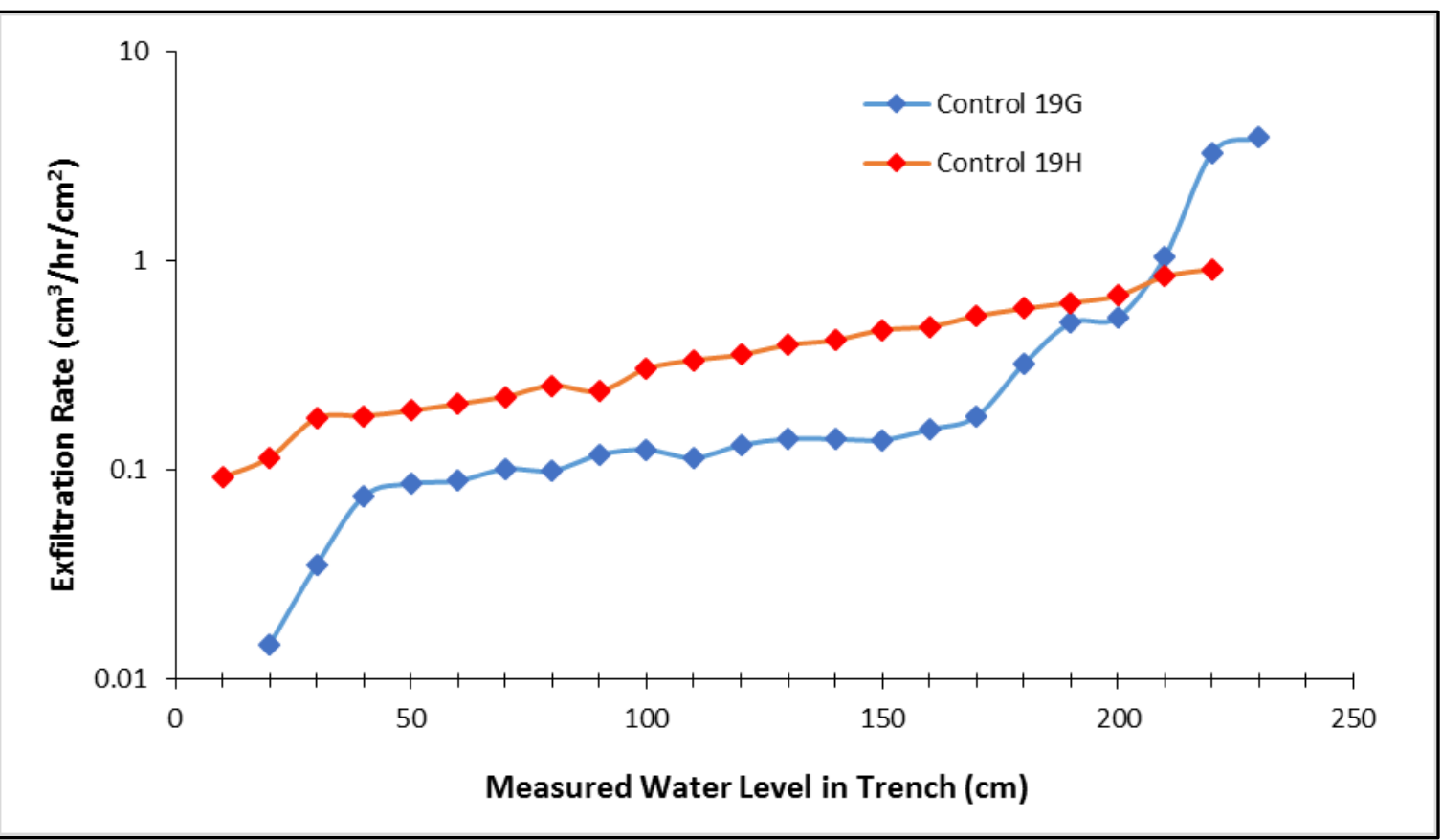

Figure 35 - Exfiiltration Rates for Controls 19G and 19H for the first Five Rainfall Events

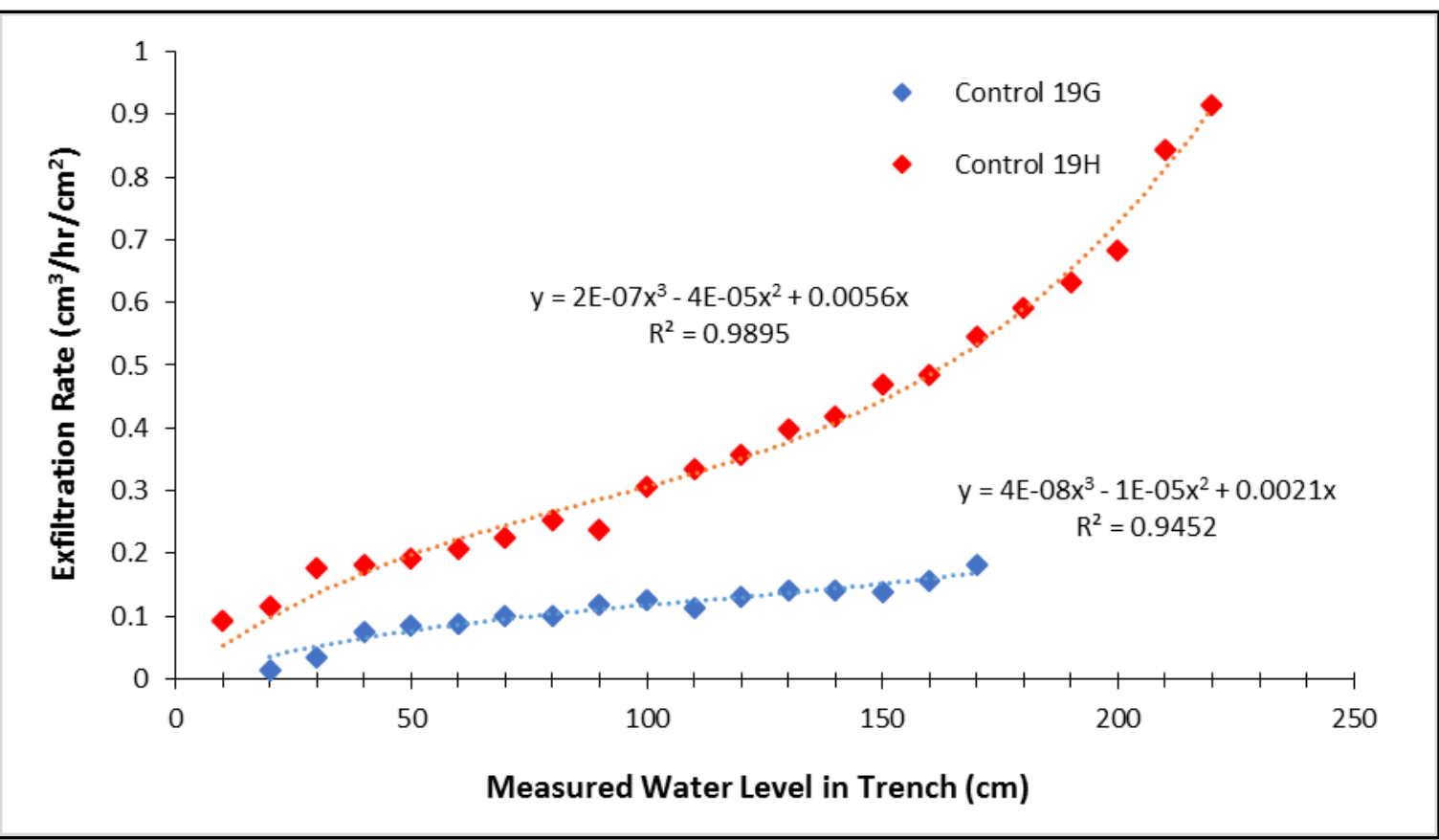

Figure 36 - An equation Can Be Established for Estimating the Exfiltration Rates based on the Water Depth inside the Trench 


\subsubsection{Infiltration Rates}

The water level rise inside the trenches, as measured by the pressure transducers, can be utilized to monitor the changes in infiltration capacity over time. This is completed by estimating the escalation (rise) and infiltration rates, while considering the inter-event exfiltration. Figure 37 shows the changes of water level for both controls $19 \mathrm{G}$ and $19 \mathrm{H}$ for a specific rain event, and as it can be seen on the graph the water level drops as the precipitation continues. This is because the stormwater exfiltrates as more stormwater infiltrates into the GI control and sometimes for the lower intensities of rainfall, the exfiltrated volume could exceed the infiltrated volume which results in a decrease of measured water level. This is called the intra-event exfiltration which should be considered while estimating the infiltration performance.

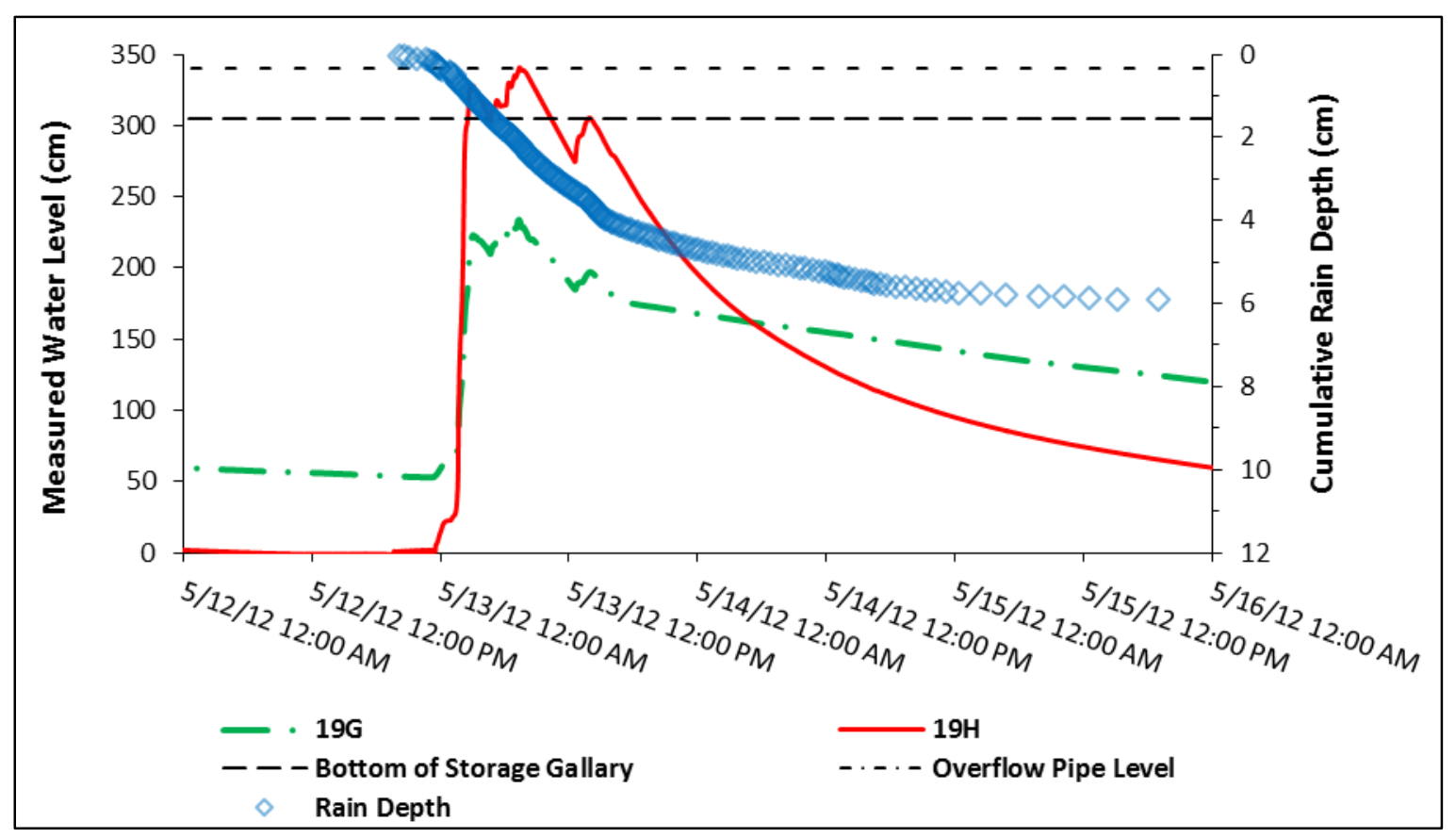

Figure 37 - Intra-Event Exfiltration for Controls 19G and 19H 
To account for the intra-event exfiltration the escalation rates are estimated by calculating the rate at which the water level rises and adding it to the estimated recession rate for the same water level:

\section{Escalation Rate $=$ Water Level Rise Rate + Recession Rate}

It must be noted that the infiltration performance of the permeable pavement system is greatly affected by the rainfall intensities and also decreases once the surface of permeable pavement is clogged. To better investigate the infiltration performance of controls $19 \mathrm{G}$ and $19 \mathrm{H}$ the water level rise between $95 \mathrm{~cm}(\sim 3 \mathrm{feet})$ and $125 \mathrm{~cm}(\sim 4$ feet $)$ depths from both controls were identified and the escalation rates were calculated for these levels. This water level range was intentionally selected because it would provide a rich data set during the two year study period. The calculated escalation rates versus the average 15-min rainfall intensities are plotted in Figure 38. As shown on the graphs a linear relationship is observed between the escalation rates and rainfall intensities and the data points can be categorized into two groups: clogged and unclogged. 

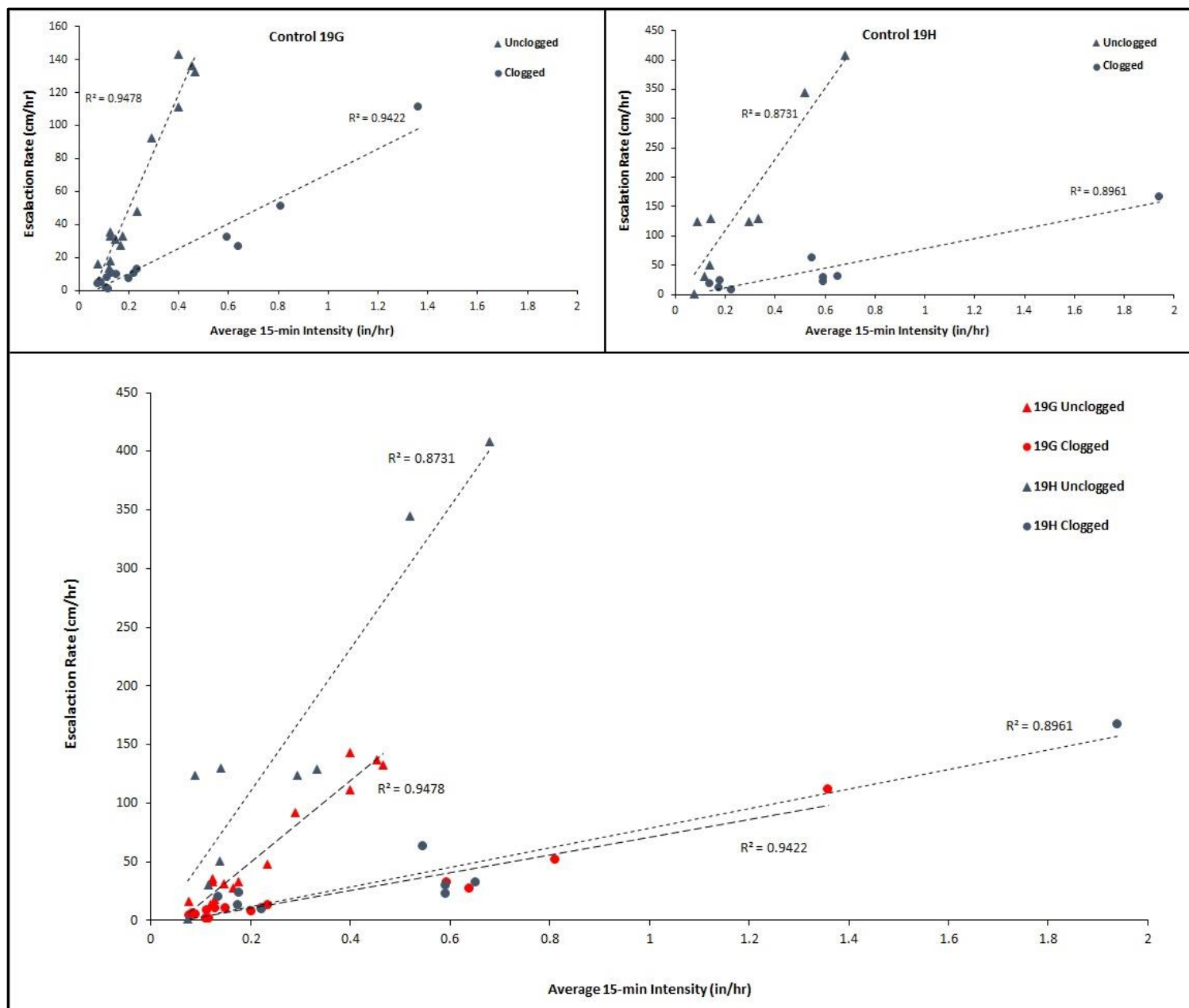

Figure 38 - Escalation Rates versus Average 15-min Intensities for Controls 19G and 19H

Similarly to exfiltration rates, infiltration rates can be calculated for the volume of infiltrated stormwater per infiltration area:

$$
I R_{Z}=\frac{\frac{\text { Volume of Infiltrated Storm Water }}{\text { Infiltration time }}}{\text { Infiltration Surface Area }}=\frac{\frac{n \times 30 \mathrm{~cm} \times x y}{t_{z-15} \mathrm{~cm}-t_{z+15 \mathrm{~cm}}}}{x y}
$$

In which,

$\mathbf{I R}_{\mathbf{z}}$ infiltration rate for the water level $z$

n: the porosity of the storage layer equal to 0.4 
$\mathbf{x}$ : width of the permeable pavement strip, equal to $8 \mathrm{ft}(244 \mathrm{~cm})$ for both controls $19 G$ and $19 H$

y: length of the trench, equal to $120 \mathrm{ft}(3660 \mathrm{~cm})$ for $19 \mathrm{G}$ and $55 \mathrm{ft}(1677.5 \mathrm{~cm})$ for control $19 \mathrm{H}$

z: captured stormwater depth measured from the bottom of the trench

Figure 39 shows the infiltration rates for controls $19 \mathrm{G}$ and $19 \mathrm{H}$ and for clogged and unclogged conditions. A similar trend is observed between the calculated escalation and infiltration rates, which was expected since they are directly related to each other. Both Figures 38 and 39 show that during rain events control 19H fills up quicker compared to control 19G which indicates a greater ratio of runoff volume to control capacity for $19 \mathrm{H}$. These measured infiltration rates are up to three orders of magnitude smaller than total infiltration rate measured by manual surface infiltration tests which are further explained in Chapter 6.

For unclogged pavement blocks, the actual infiltration rates never reaches the pavement's ultimate infiltration capacity. Under such conditions the runoff volume entering into the surface of the GI control is the limiting factor. Therefore the two GI controls have differing measured infiltration rates as the GIs have differing tributary areas and associated runoff volumes per rain event. On the other hand for clogged pavement blocks, the infiltration capacity of the pavement surface is drastically decreased and the runoff volume that enters each GI control is limited by the pavement's infiltration capacity. Under this condition, the clogged pavement's capacity is the dominant factor affecting the infiltration rates and therefore both GI controls show similar infiltration performance. 

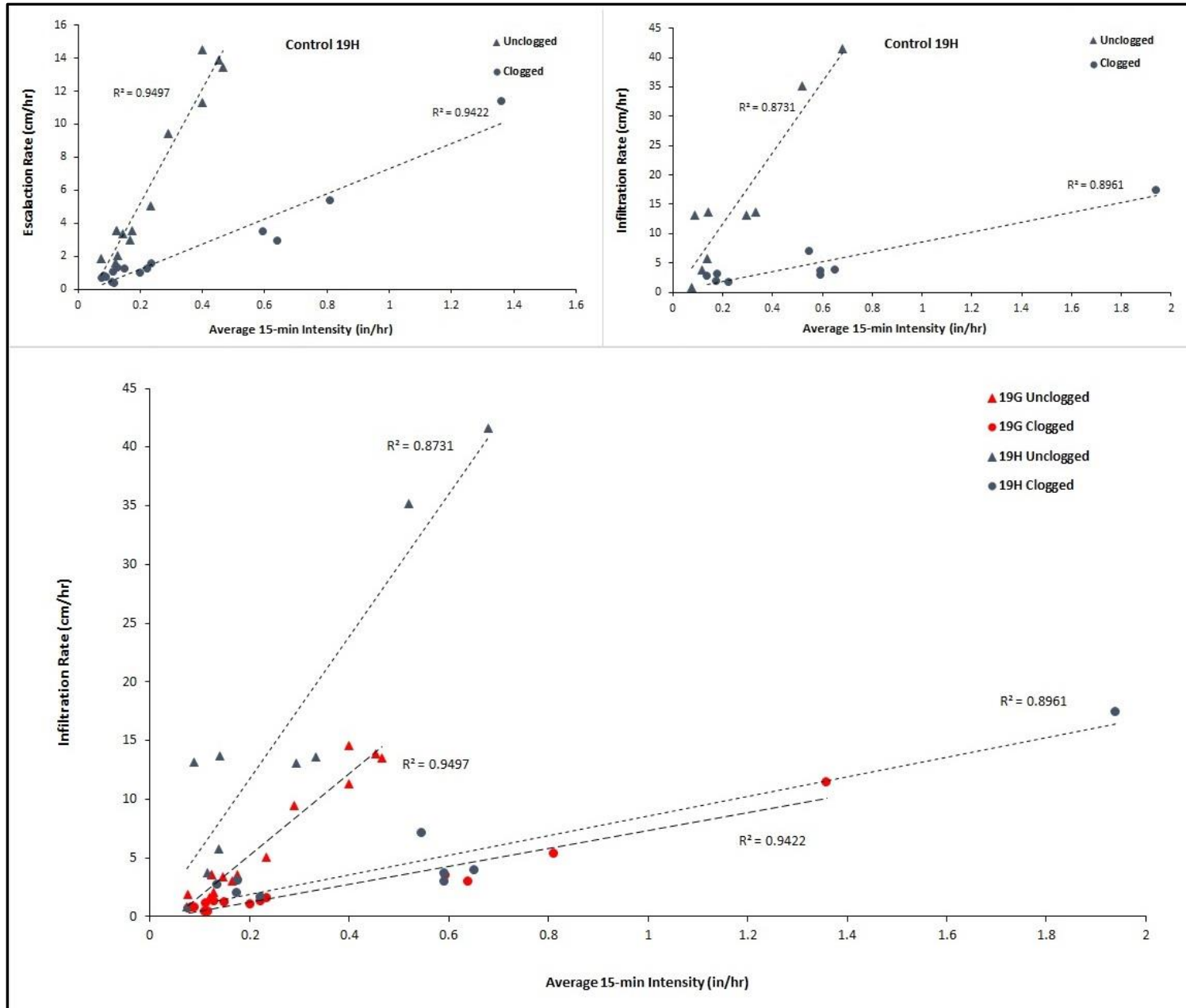

Figure 39 - Infiltration Rates versus Average 15-min Intensities for Controls 19G and 19H

\subsection{TDR Data}

Section 3.3.1 of this document explained the location of TDRs installed in each GI control. Each TDR includes two parallel stainless steel rods that are connected to a differential oscillator circuit. The two-way travel time of the electromagnetic waves, which are induced by the oscillator on the rod, varies with changing dielectric permittivity of the medium surrounding the probes. Since water is the main contributor to the bulk dielectric permittivity of the porous media, the travel time of the reflected electromagnetic waves increases with an increase of water content in the porous medium and subsequently 
decreases with a decrease of water content (Campbell Scientific 2012). Thus, the TDR data can be used to estimate the volumetric water content at specific locations within the controls. During the rainfall events, if the measured volumetric water content increases, we can infer that water is infiltrating into the GI control at that location, and that the point is unclogged. Otherwise it would either mean that the point is clogged or the runoff volume is entering the GI control at more upgradient locations.

It is noted that TDR sensors are primarily used in agricultural applications to measure the true values of volumetric water content (VWC) of soil layers. In this project, however, the TDRs are installed in stones and thus their recorded values do not reflect true VWC of their environment. As such these measured VWC values are used to only provide comparison of water content in different locations within each GI control. The TDR data has been very useful in understanding the pattern of surface clogging progression and also in evaluating the effectiveness of each surface maintenance treatment.

Figure 40 shows the TDR responses for the five TDRs installed along the curbside of control 19G and for the first four rainfall events. As shown on this figure, the peak responses moved from the most upgradient TDR to the TDR located at 20 feet from the upgradient edge by the end of the fourth rain event. This indicates the progression of surface clogging from the upgradient side to the downgradient side which subsequently shifts the point of stormwater infiltration along the surface. This is similar to the pattern that was observed by the recorded data from pressure transducers, see Figures 28 and 29. Figure 41 shows the measured maximum volumetric water contents for each rainfall event recorded by the curbside TDRs for control 19G, which better shows the movement of 
clogging on the surface of the GI control $19 \mathrm{G}$ to the downgradient side during the first fourteen rainfall events. 


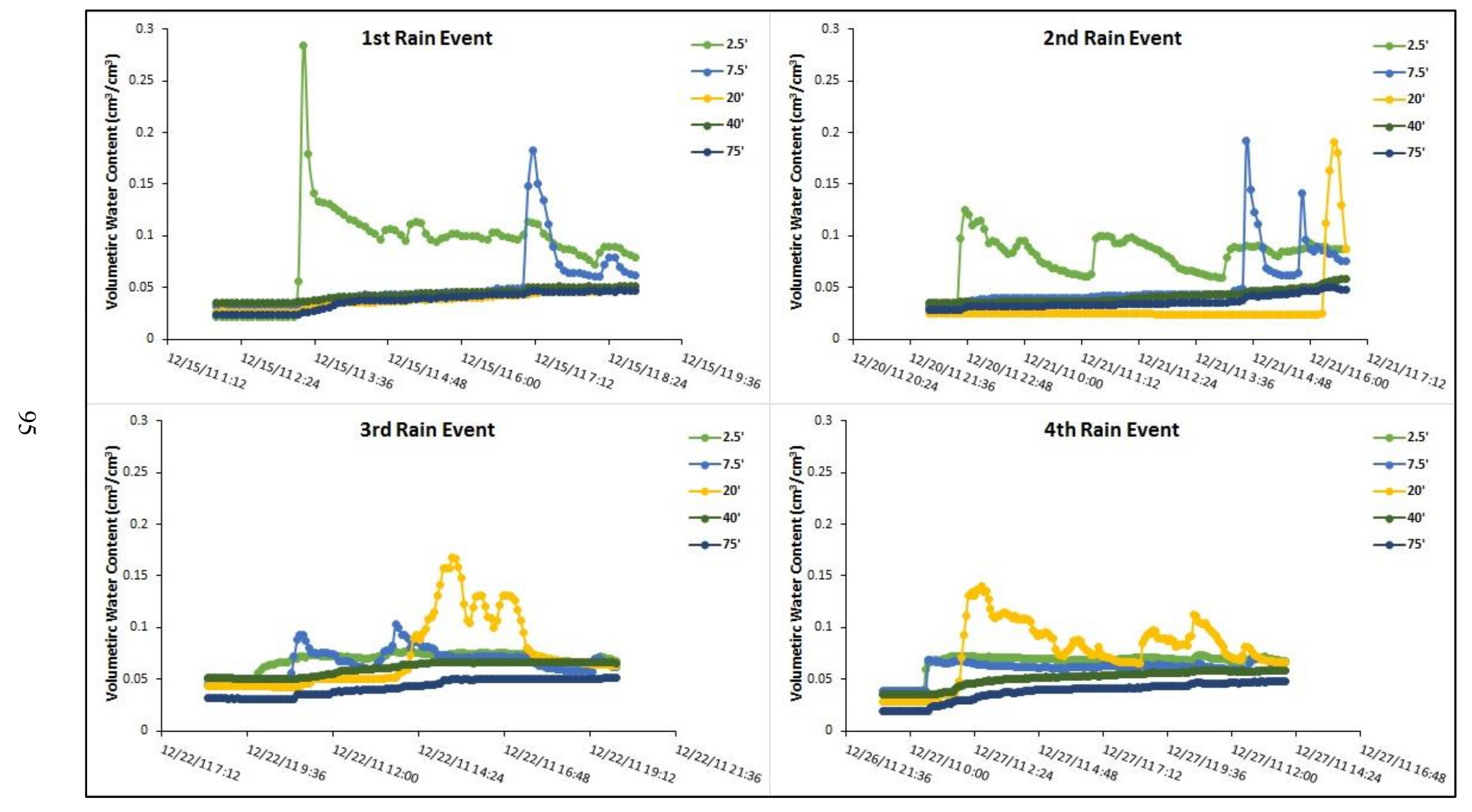

Figure 40 - Curbside TDR Responses for the First Four Rainfall Events, Control 19G 


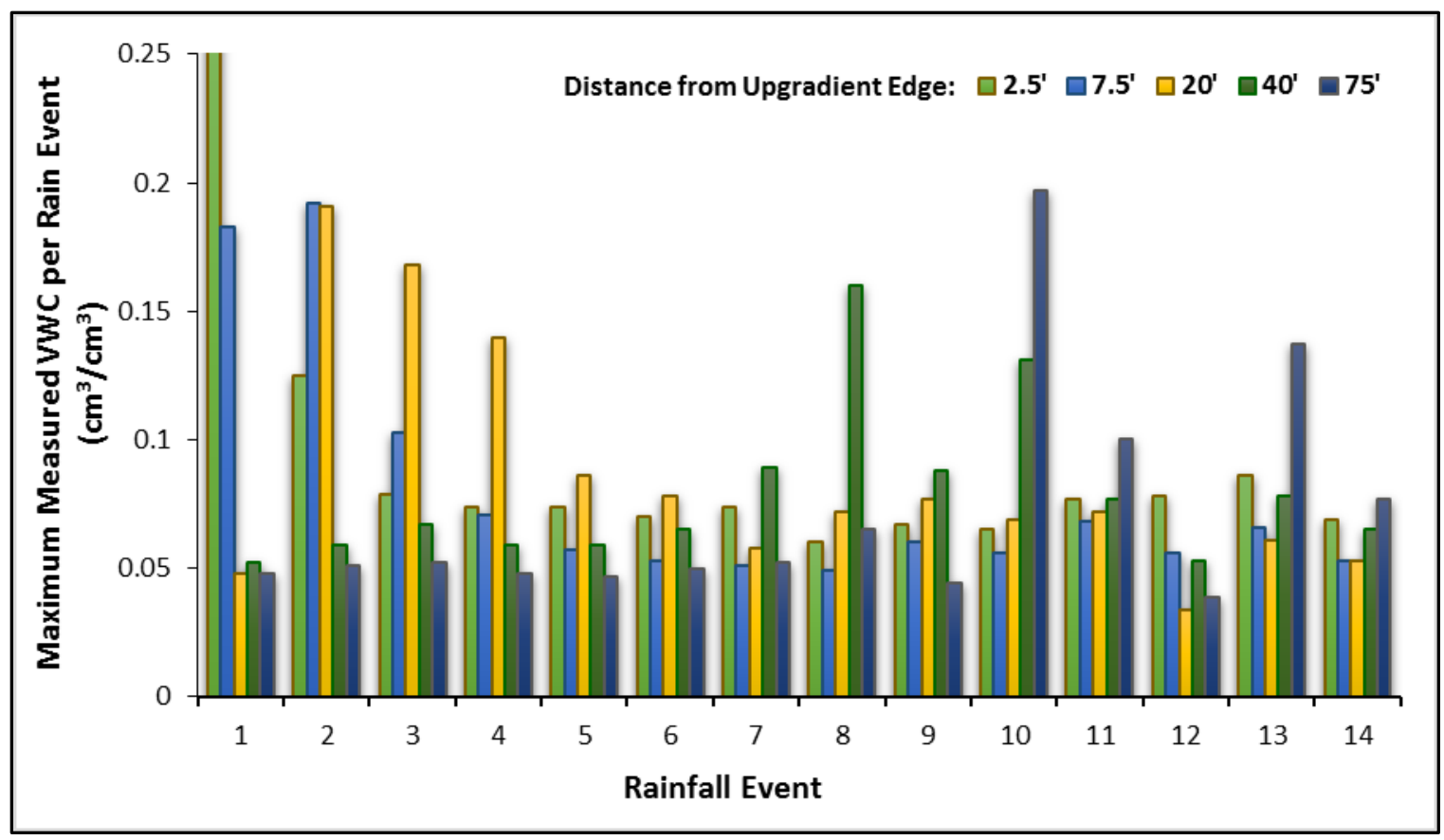

Figure 41 - Maximum Recorded VWCs by Curbside TDRs versus Time (Event Number), Control $19 G$

To better understand the clogging progression along the length of control 19G, maximum VWC values recorded by curbside TDRs are plotted against the cumulative rainfall after the installation of control 19G, see Figure 42. The graph suggests a constant clogging rate along the curbside of the permeable pavement surface. It must be noted that for evaluating the clogging progression, only the TDR data from control $19 \mathrm{G}$ is analyzed not the data from control $19 \mathrm{H}$. The data from $19 \mathrm{H}$ was not included in the analysis because first, control 19G had a shorter overall length and thus only had four imbedded TDRs. And second, the location of the most upgradient TDR in $19 \mathrm{H}$ had to be moved out of the desired location due to construction obstructions. The most upgradient TDR in 19H was moved 6 inches from the curb to 12 inches because of a protruding concrete. Thus the useable remaining three TDRs along the curbside of control $19 \mathrm{H}$ didn't provide enough data to establish valid linear regressions between measured values. Although these three TDRs do 
suggest the same pattern of clogging progression as observed in control 19G, the available data cannot validate the observations, see Figure 43. Secondly control $19 \mathrm{H}$ has had fewer maintenance treatments than control $19 \mathrm{G}$ so it doesn't provide sufficient information regarding the different maintenance types and their effectiveness, which is further explained in chapter 6 .

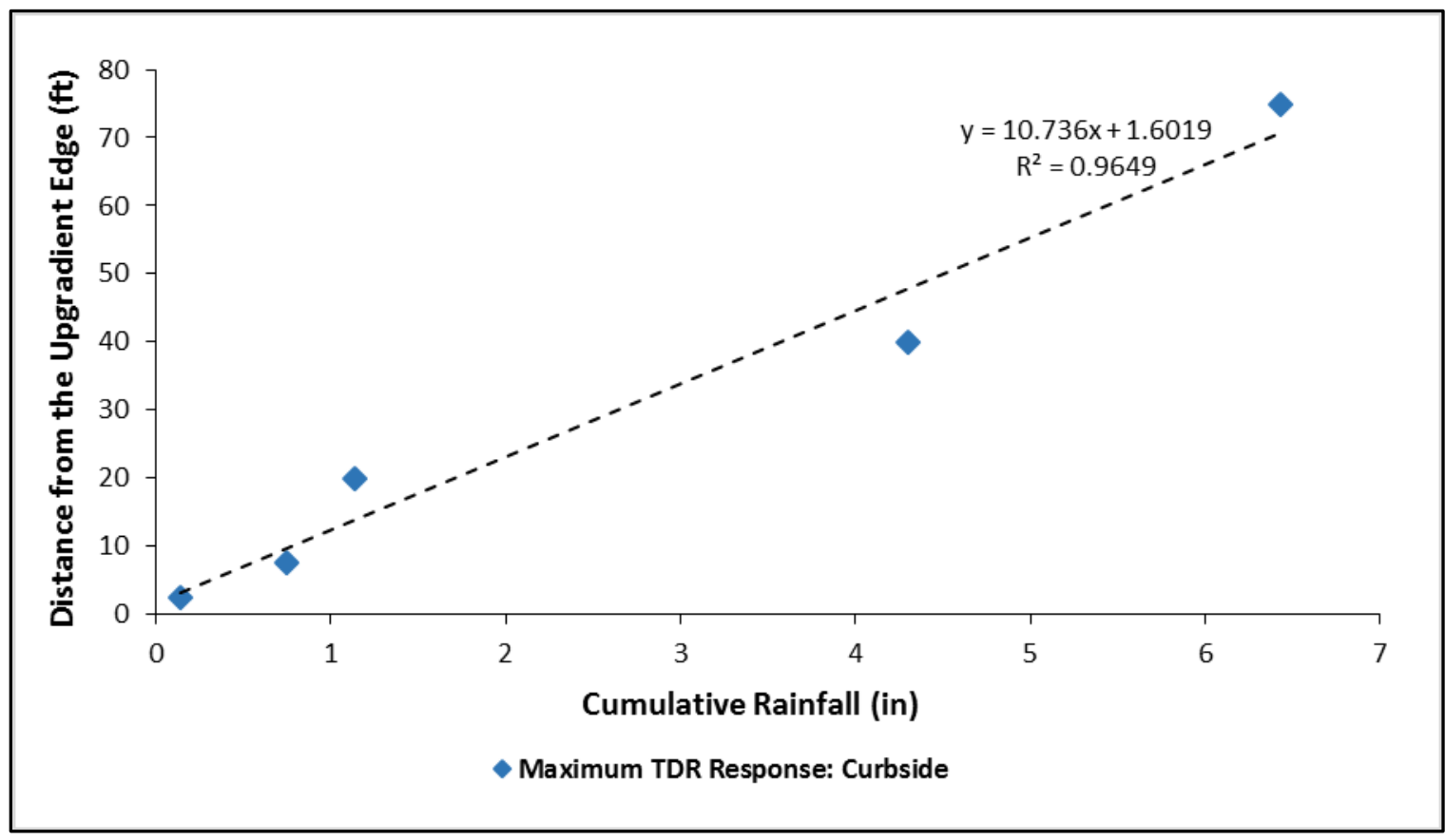

Figure 42 - Maximum TDRs' Responses along Curbside versus Cumulative Rainfall, Control 19G

\subsubsection{Surface Clogging Progression Rate}

In the previous analyses it was observed that clogging along the curbside had a linear relationship with cumulative rainfall. As such a linear regression analysis between the TDRs' locations and cumulative rainfall was used to define a threshold TDR response value that would indicate clogged conditions. The analysis was performed by identifying the first and the last time each curbside TDR recorded a value greater than the selected threshold. The time period for this analysis was from immediately after construction of 
control 19G's up to its initial surface maintenance (mid-December 2011 to mid-March 2012). During this event window, six thresholds were selected and tested for measured VWC values $\left(0.08,0.09,0.10,0.11,0.12\right.$, and $\left.0.14 \mathrm{~cm}^{3} / \mathrm{cm}^{3}\right)$. It must be noted that the methodology for this analysis was initially suggested and explained by Brown and Borst (2012) by selecting three threshold values (Brown and Borst 2013).

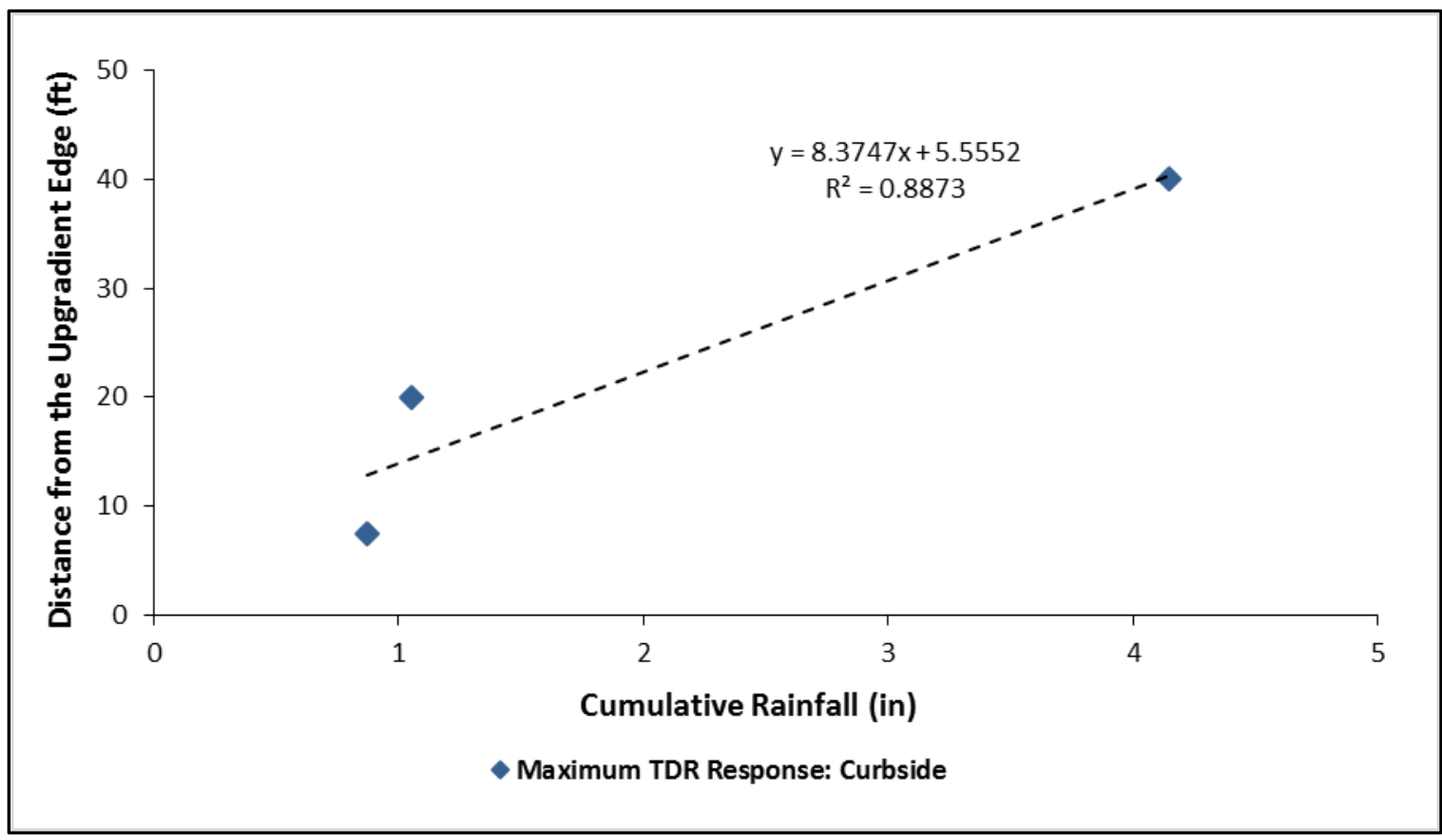

Figure 43 - Maximum TDRs' Responses along Curbside versus Cumulative Rainfall, Control 19H

Figure 44 illustrates the results of comparing the progression of initial clogging along the upgradient edge to the cumulative rainfall. As can be seen on the graphs from Figure 44, by increasing the thresholds values the distance between the two linear trend lines decreases. The thresholds between $0.10 \mathrm{~cm}^{3} / \mathrm{cm}^{3}$ to $0.12 \mathrm{~cm}^{3} / \mathrm{cm}^{3}$ seem more suitable than other selected values since they provide better coefficients of determination (Rsquared) values. It is suggested to use $0.12 \mathrm{~cm}^{3} / \mathrm{cm}^{3}$ and $0.1 \mathrm{~cm}^{3} / \mathrm{cm}^{3}$ as thresholds in which the first and last occurrences exceeding the threshold happen, respectively, see Figure 45. 


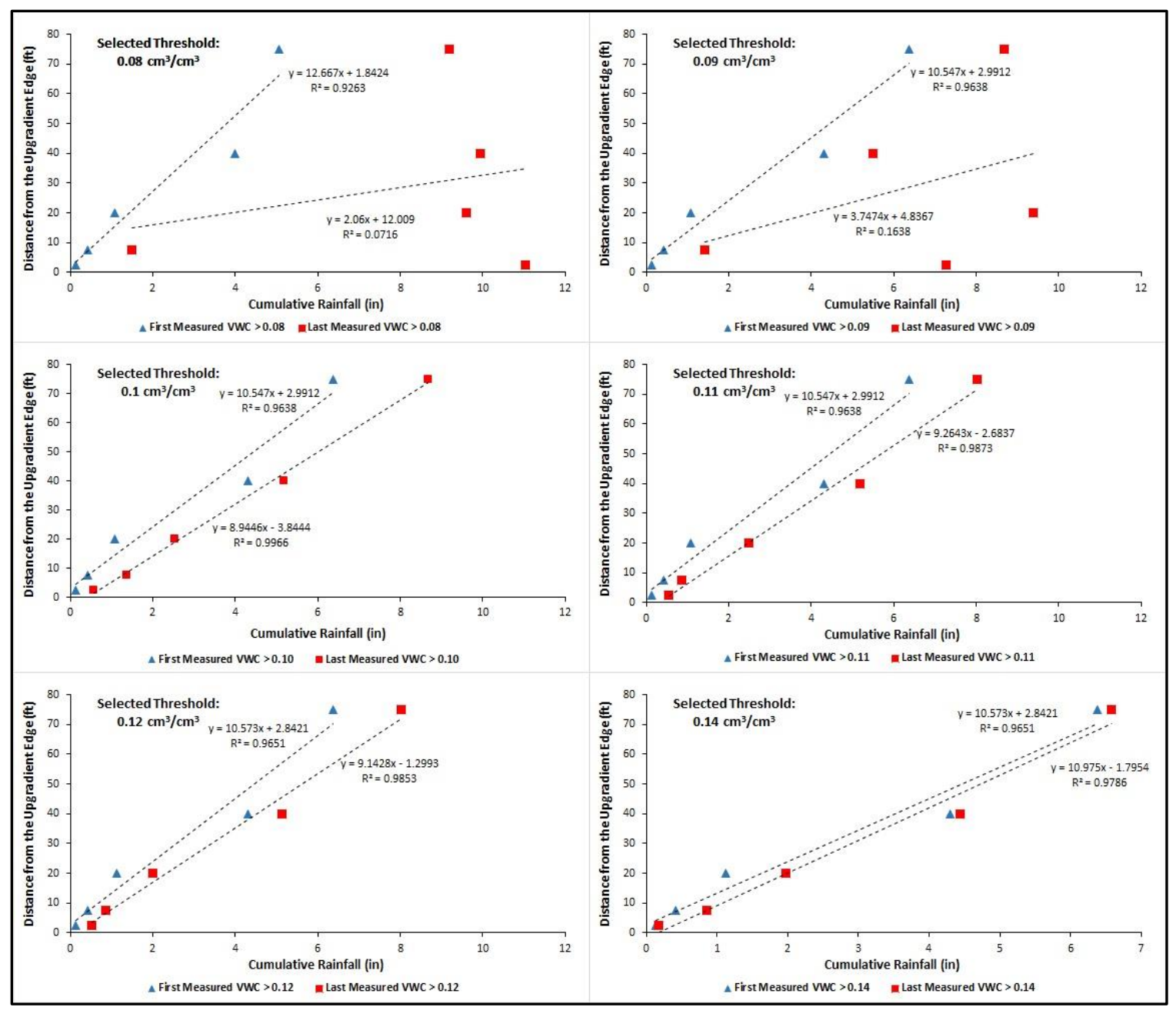

Figure 44 - Selecting a Threshold for Determining the Surface Clogging Rate

Since the lines with last occurrences exceeding the selected threshold indicate actual clogging for a location, it seems more fitting to use this trend line and with a 0.1 $\mathrm{cm}^{3} / \mathrm{cm}^{3}$ value as the threshold. By doing so the clogging rate on the surface of control 19G can be determined as approximately $9 \mathrm{ft} / \mathrm{in}$ ( $9 \mathrm{ft}$ per an inch of rainfall). This rate matches the clogging rate observed by visual inspections and also the one derived from modeling effort explained in Chapter 5 of this document. 


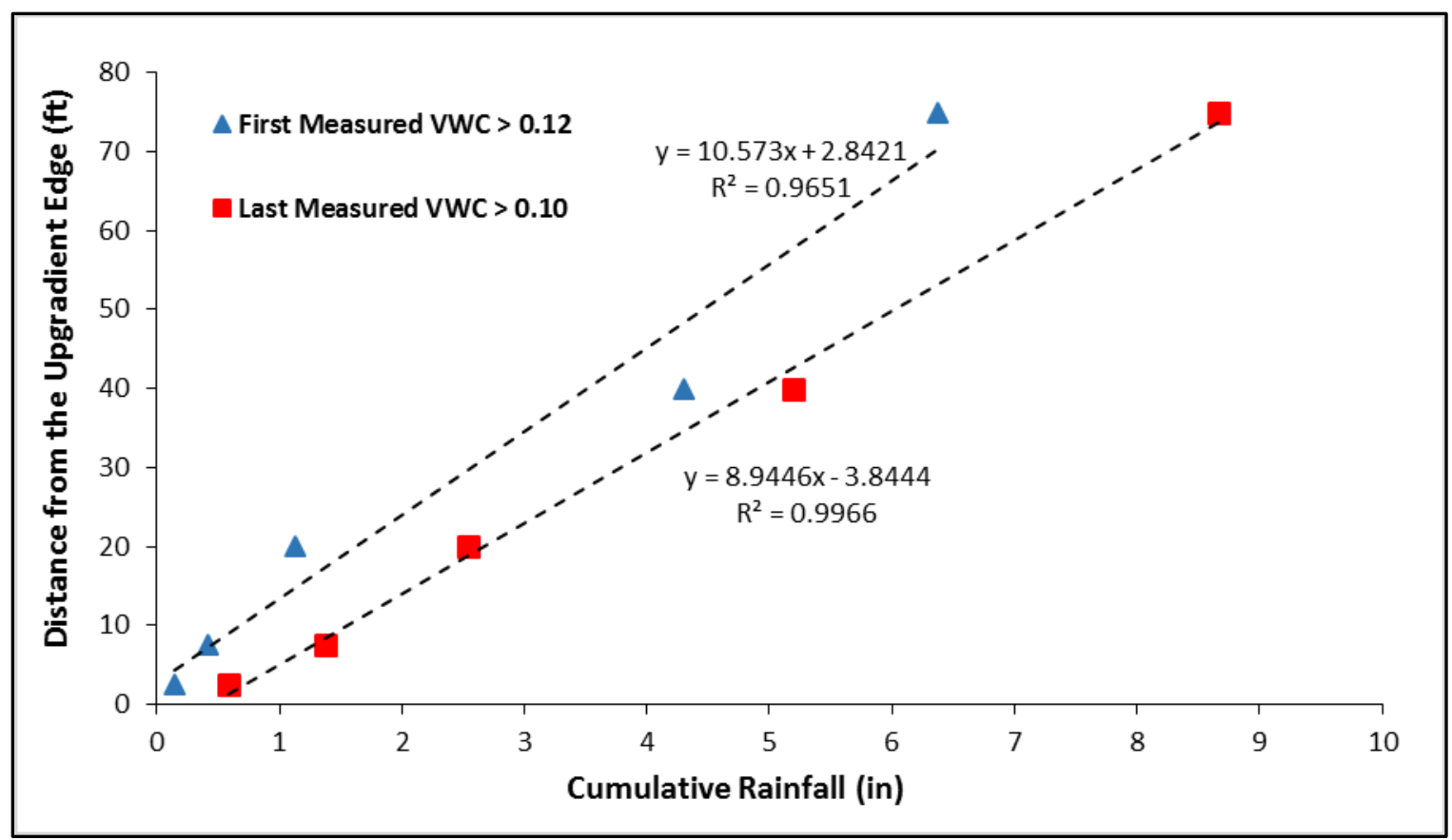

Figure 45 - Suggested TDR Thresholds for Determining the Surface Clogging Rate, Control 19G

The uneven distance between the two lines in Figure 45 indicate that length of surface runoff on the permeable pavement surface increases as clogging advances toward the downgradient edge. This is expected since downgradient locations were probably partially clogged due to the direct rainfall and runoff flow from the street side (crown of the road) before the longitudinal flow from upgradient locations reaches them. Other factor that could result in faster first occurrences exceeding the selected threshold is rainfall intensity, as for more intense rainfalls the length of runoff flow before infiltration increases and therefor first occurrences exceeding the threshold are recorded faster.

While this analysis method and the determined clogging rate value seem efficient for identifying the pattern of clogging progression on the surface of control 19G, care must be taken in interpreting the results and assigning them to other applications of permeable pavement systems. Factors such as the pattern of permeable pavement blocks, porosity of 
the media that the TDRs are installed in, as well as their distance to the infiltration level (which here is the surface of permeable pavement system) could potentially affect the most desirable threshold values. Also clogging rates for different locations could vary greatly due factors such as slope of the permeable pavement system and the amount of fine sediments and organic material existent in runoff flow for that specific location. The TDR analysis explained in this section is most beneficial in evaluating the effectiveness of maintenance treatments which is explained in Chapter 6.

\subsubsection{Width of Runoff Flow}

Visual inspections during rain events confirmed that the active runoff flow on the permeable pavements before infiltration hardly exceeded half of the control's surface, see Figure 46. It was further observed that width of runoff flow was wider on clogged sections and decreased downgradient as it traversed unclogged sections.

Based on visual observation during rain events, it is evident that the center of the permeable pavement sections are clogging in a pattern somewhat similar to the pavement edges. However, the first surface maintenance on control 19G was conducted before clogging completely advanced toward the downgradient edge on the middle of the permeable pavement. 


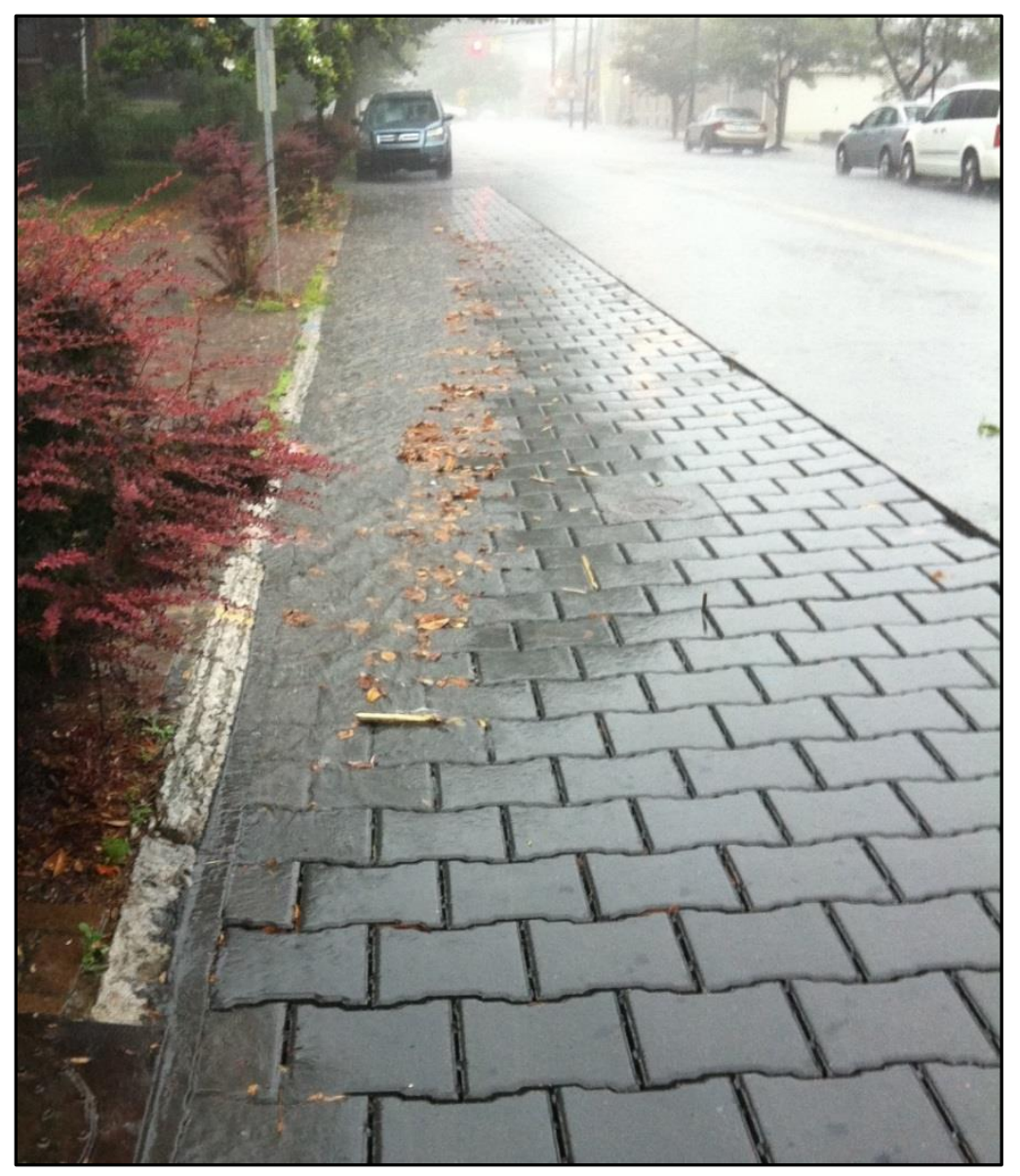

Figure 46 - Runoff Flow during a Rain Event, Control 19G

While the center of the controls did not clog as extensively as the curb edge, Figure 47 shows the first occurrences of the TDR data exceeding the $0.12 \mathrm{~cm}^{3} / \mathrm{cm}^{3}$ threshold. The TDR at the center of permeable pavement and $75 \mathrm{ft}$ from the upgradient edge, didn't record any measurements exceeding the 0.1 or $0.12 \mathrm{~cm}^{3} / \mathrm{cm}^{3}$ thresholds and is excluded from the graph on Figure 47. The slopes of linear regressions shown on Figure 47 indicate the progression of clogging rate at middle and curbside of the pavement surface based on the first occurrences of the TDR data exceeding the selected threshold (per an inch of cumulative rainfall). 


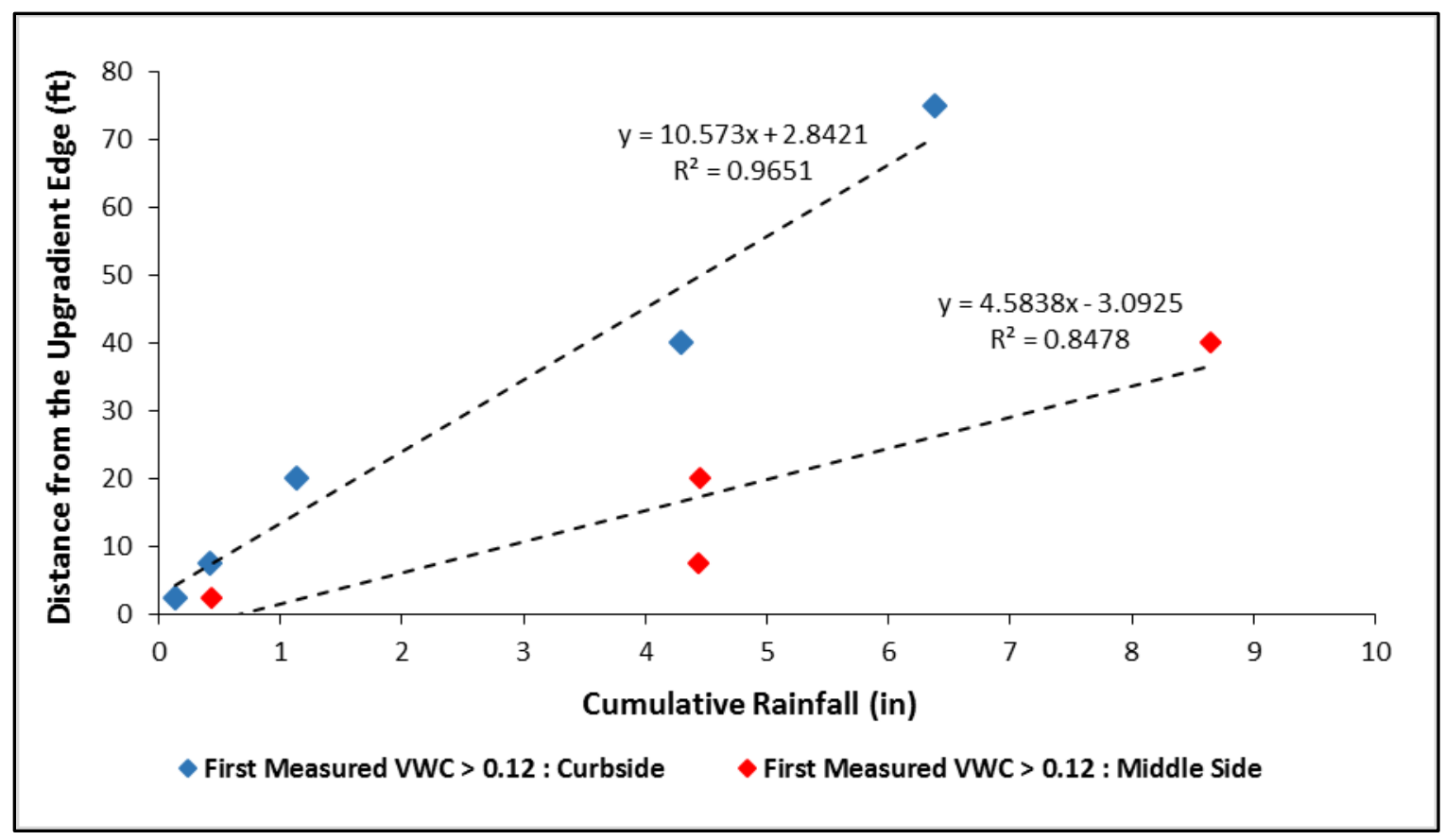

Figure 47 - Comparison of Clogging Rate on Curbside and Middle, Control 19G

Figure 47 confirms that the clogging at the middle of the permeable pavement surface occurs after the curbside. Based on the first occurrences of the TDR data exceeding the selected threshold, the clogging has a slower rate of progress toward downgradient side (4.6 ft/in compared to the rate of $10.6 \mathrm{ft} / \mathrm{in}$ ) than the curbside. It must be noted that the active width of runoff flow and clogging rate at middle of permeable pavement surface is a factor of longitudinal and cross sectional slopes of the pavement, as well as the drainage ratio and rainfall intensities, and would probably be different for other applications of permeable pavements.

The TDRs located adjacent to the street edge did not record any values greater than 0.1 or $0.12 \mathrm{~cm} 3 / \mathrm{cm} 3$ during this time period. Thus, a clogging analysis for the street edge could not be performed. 


\subsection{Changes in Exfiltration Rates and Contributing Factors}

The method for estimating the exfiltration rates for controls $19 \mathrm{G}$ and $19 \mathrm{H}$ were explained in Section 4.2.1. In this section the observed changes in values of exfiltration rates over the course of this study are reviewed. A total of 58 rainfall events were selected for calculating the exfiltration rates during the study period by use of equation 4.1. It must be noted that $20-\mathrm{cm}$ intervals were selected instead of $10-\mathrm{cm}$ intervals to make the analysis manageable. Table 13 summarizes the water levels and the number of rainfall events for which the exfiltration rates are calculated.

Table 14 - Water Levels and Number of Calculated Exfiltration Rates, Controls 19G and 19H

\begin{tabular}{cccc}
\hline \multicolumn{2}{c}{ Control 19G } & \multicolumn{2}{c}{ Control 19H } \\
\hline $\begin{array}{c}\text { Water Depth } \\
(\mathrm{cm})\end{array}$ & $\begin{array}{c}\text { Number of Exfiltration } \\
\text { Rate Measurements }\end{array}$ & $\begin{array}{c}\text { Water Depth } \\
(\mathrm{cm})\end{array}$ & $\begin{array}{c}\text { Number of Exfiltration } \\
\text { Rate Measurements }\end{array}$ \\
\hline 55 & 21 & 15 & 41 \\
\hline 75 & 27 & 35 & 52 \\
\hline 95 & 40 & 55 & 53 \\
\hline 115 & 50 & 75 & 44 \\
\hline 135 & 42 & 95 & 30 \\
\hline 155 & 34 & 115 & 25 \\
\hline 175 & 22 & 135 & 17 \\
\hline
\end{tabular}

Calculated exfiltration rates for controls $19 \mathrm{G}$ and $19 \mathrm{H}$ over the 2-year study period are shown in Figures 48 and 49. Since these figures suggested seasonal changes of exfiltration rates, recorded temperatures inside the GI controls are also included in these graphs. The temperature values are recorded for three different depth: bottom of the trench (average of values measured by the pressure transducers), mid-depth of the trench (average of values measured by two thermistors), and at the interface of \#3 and \#57 stone layers (average of values measured by the surface TDRs). As it can be seen, the measured temperatures show 
a temporal lag with depth. Measured temperatures at the bottom of the trench show less variations thought the year compared to temperatures at shallower depths.

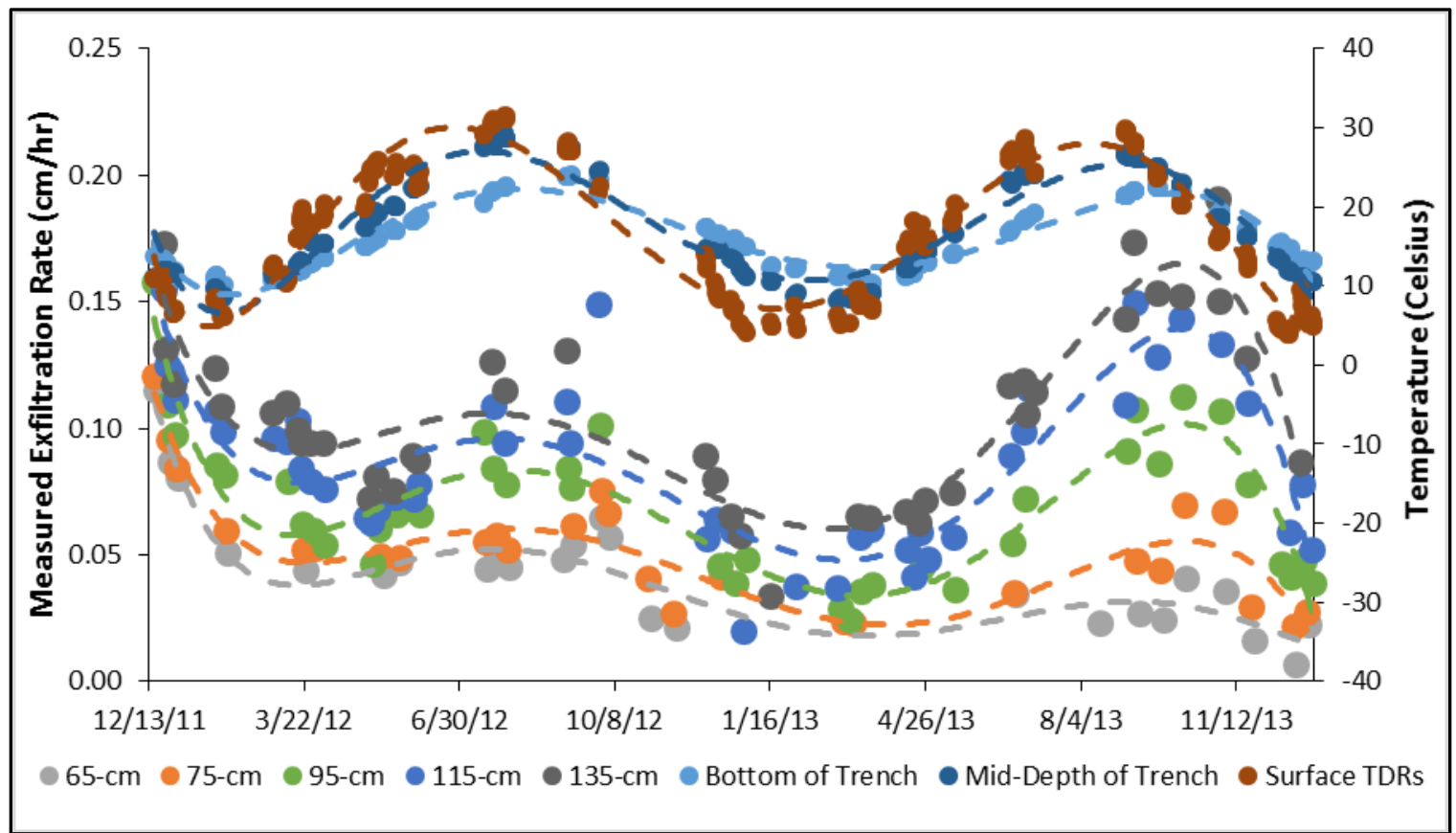

Figure 48 - Measured Exfiltration Rates and Recorded Sub-Surface Temperatures for the Study Period, Control 19G 


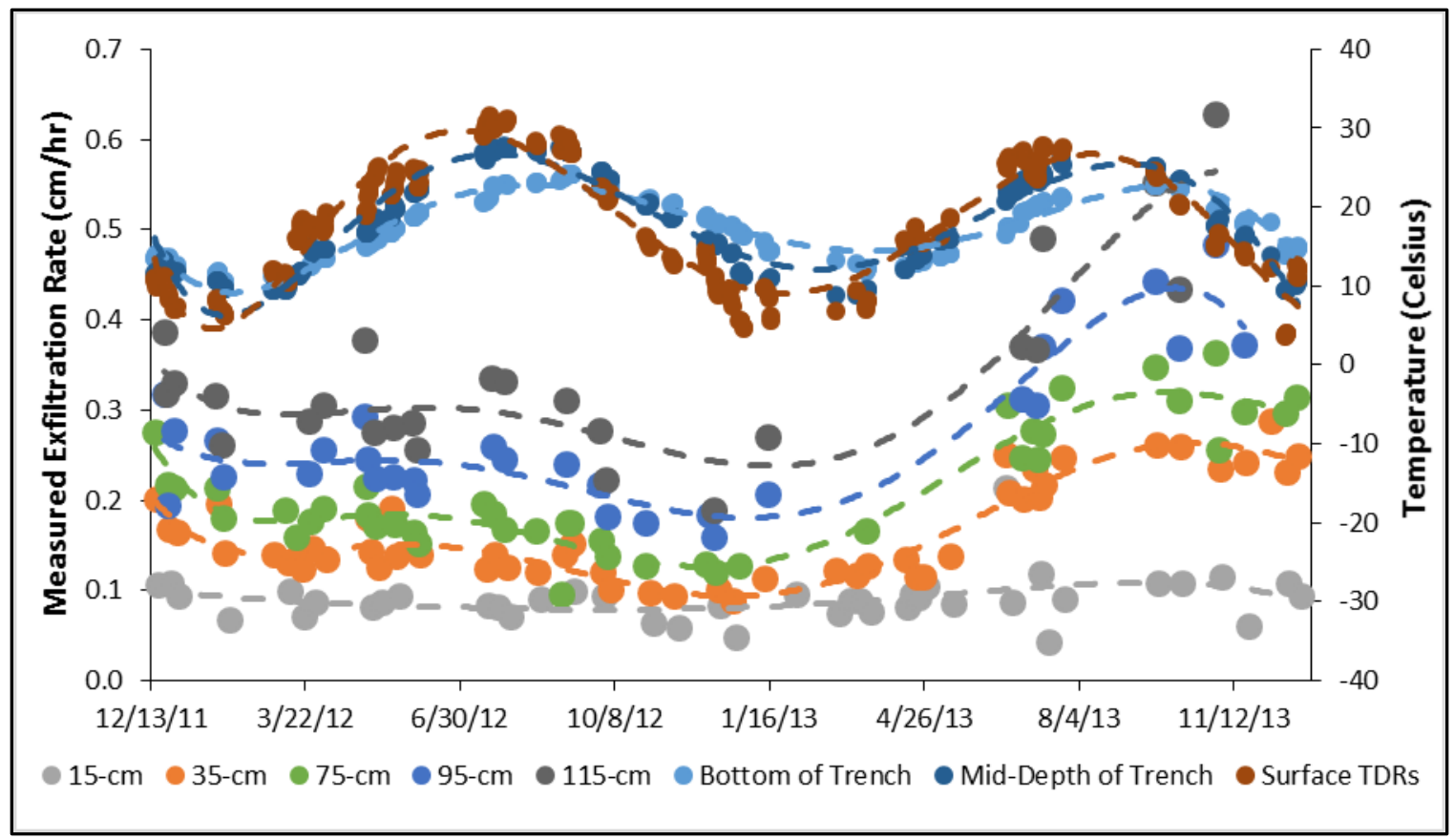

Figure 49 - Measured Exfiltration Rates and Recorded Sub-Surface Temperatures for the Study Period, Control $19 \mathrm{H}$

From Figures 48 and 49 it can be deducted that the exfiltration rates from both GI controls are showing fluctuations throughout each year with having their maximum values in summer (between August and October) and their minimum values in Winter (between December and March). The general trend of changes in exfiltration rates seems like to follow the trend of changes of temperature values measured at the bottom of the trench. Also an initial significant decrease in exfiltration rates after the installation of the GI controls, and with age, is observed, which is more evident in control 19G than $19 \mathrm{H}$. Figures 50 and 51 show the average exfiltration rate values for each year. It must be noted that the values for year 2011 includes only 3 rainfall events immediately after construction of the GI controls and during the month of December. The average temperatures for all data points in each year are also calculated and presented in the secondary axis. It seems like both GI controls have experienced decreases in their average exfiltration values in 2012 , 
despite the increase of temperature at the bottom of the trenches. During 2013, control 19G didn't show any significant decreases while control $19 \mathrm{H}$ even experienced increases in its exfiltration rates.

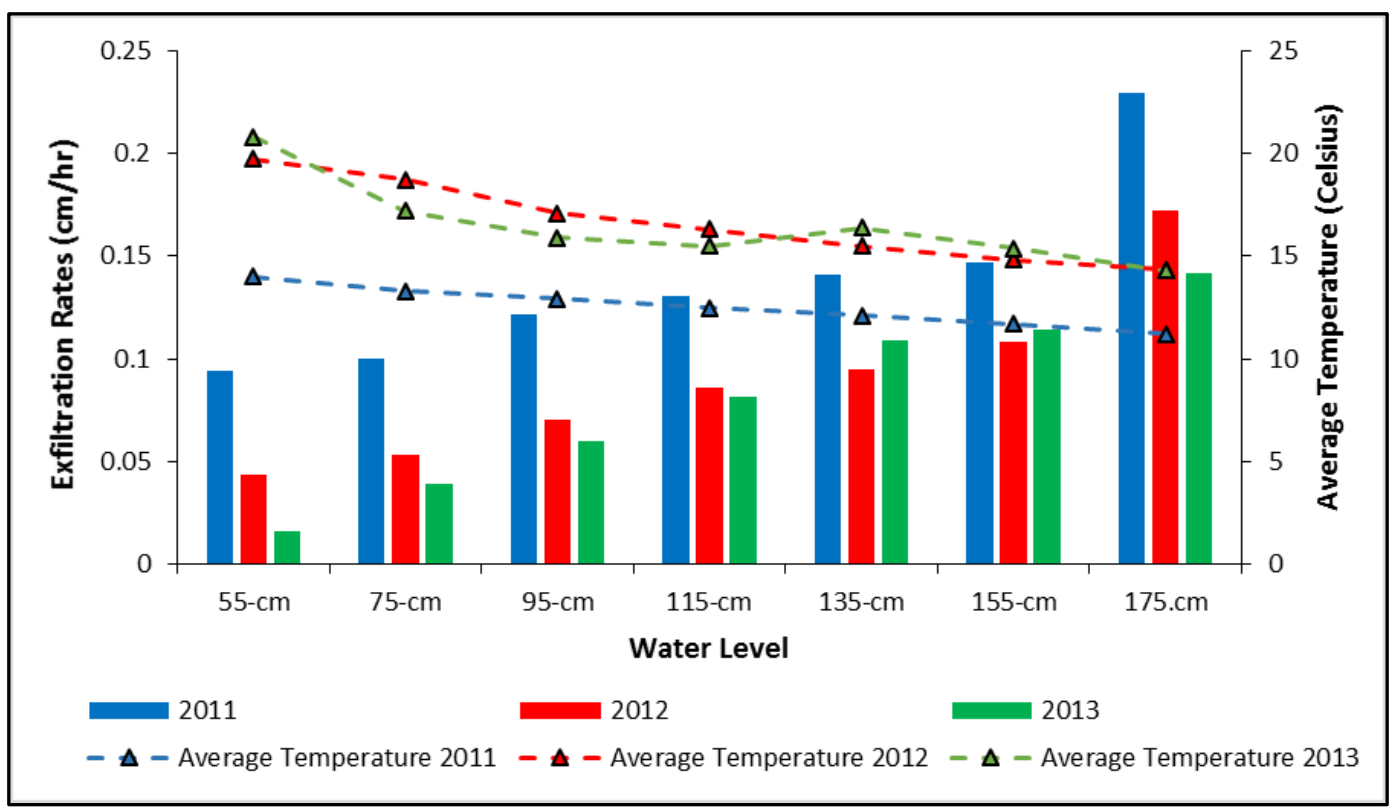

Figure 50 - Average Exfiltration Values for years 2011, 2012, and 2013 for Control 19G

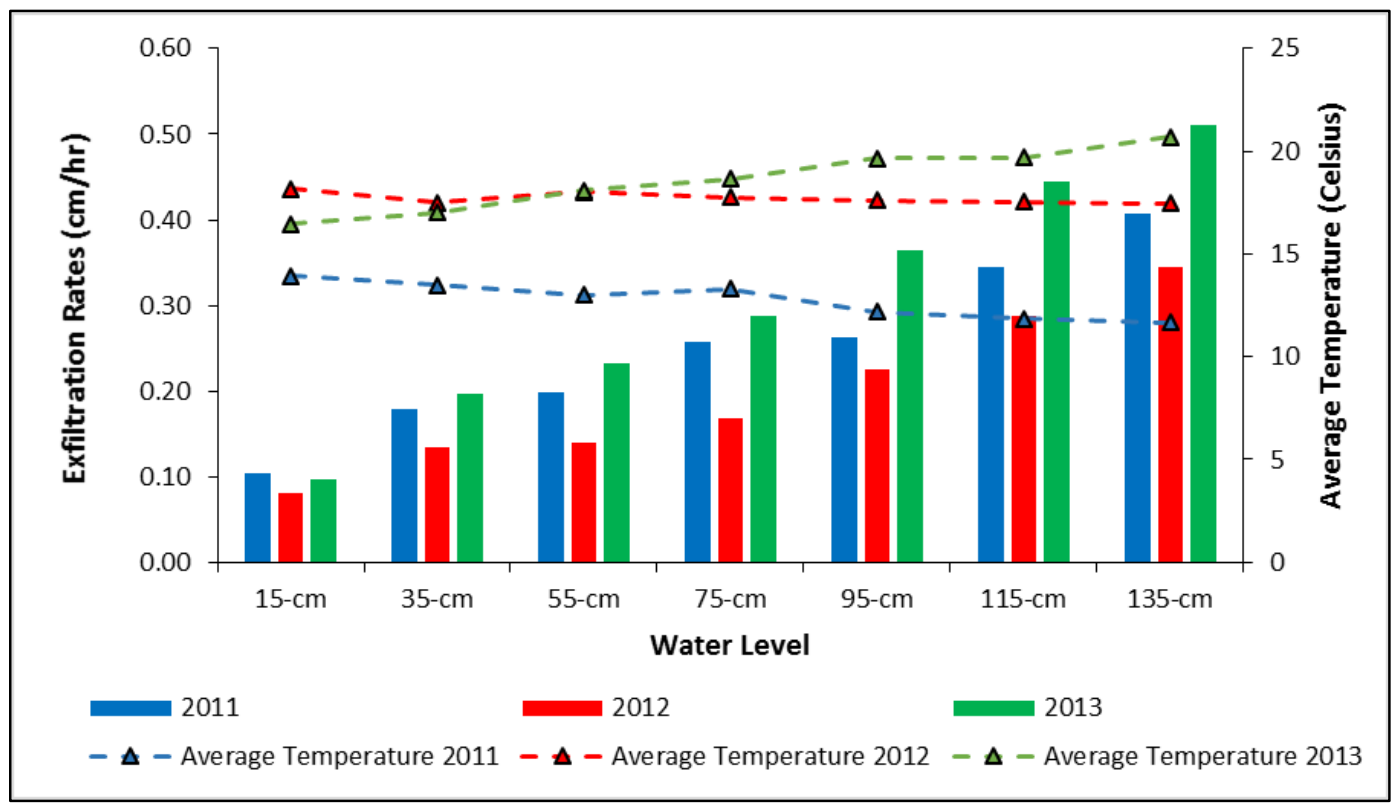

Figure 51 - Average Exfiltration Values for years 2011, 2012, and 2013 for Control 19H 
To compare the significance of observed differences between the exfiltration rates from each year, three pair of Student's t-tests are performed for each water level and between values from 2011 and 2012, 2012 and 2013, and between 2011 and 2013. The results are provided separately for controls $19 \mathrm{G}$ and $19 \mathrm{H}$ in Tables 15 and 16 . It must be noted that since the calculated exfiltration rate values didn't show a normal distribution, performing analysis of variance (ANOVA) tests to compare the changes in exfiltration rates were not possible and therefore a nonparametric test (Kruskal-Wallis test) was selected. The p-values showing significant differences $(\mathrm{P}<0.05)$ are shown in bold.

Table 15 - Kruskal-Wallis Test Results Comparing the Exfiltration Rate Values in Each Year, Control 19G

\begin{tabular}{|c|c|c|c|c|c|c|c|c|c|}
\hline \multirow{3}{*}{$\begin{array}{l}\text { Water } \\
\text { Level } \\
(\mathrm{cm})\end{array}$} & \multicolumn{6}{|c|}{ Exfiltration Rates $(\mathrm{cm} / \mathrm{hr})$} & \multicolumn{3}{|c|}{ Comparison Between } \\
\hline & \multicolumn{2}{|c|}{2011} & \multicolumn{2}{|c|}{2012} & \multicolumn{2}{|c|}{2013} & \multirow{2}{*}{$\begin{array}{c}2011 \\
\& \\
2012 \\
P \\
\text { Value }\end{array}$} & \multirow{2}{*}{$\begin{array}{c}2011 \\
\& \\
2013 \\
P \\
\text { Value }\end{array}$} & \multirow{2}{*}{$\begin{array}{c}2012 \\
\& \\
2013 \\
P \\
\text { Value }\end{array}$} \\
\hline & Mean & St. Dev. & Mean & St. Dev. & Mean & St. Dev. & & & \\
\hline 55 & $\begin{array}{c}0.0943 \\
(\mathrm{n}=2)\end{array}$ & 0.0120 & $\begin{array}{c}0.044 \\
(n=13)\end{array}$ & 0.0138 & $\begin{array}{c}0.01641 \\
(n=6)\end{array}$ & 0.00661 & 0.027 & 0.046 & 0.001 \\
\hline 75 & $\begin{array}{c}0.1002 \\
(n=3)\end{array}$ & 0.0186 & $\begin{array}{l}0.0530 \\
(\mathrm{n}=14)\end{array}$ & 0.0119 & $\begin{array}{l}0.0390 \\
(\mathrm{n}=10)\end{array}$ & 0.0178 & 0.004 & 0.011 & 0.040 \\
\hline 95 & $\begin{array}{c}0.1216 \\
(n=3)\end{array}$ & 0.0323 & $\begin{array}{l}0.0706 \\
(n=18)\end{array}$ & 0.0178 & $\begin{array}{l}0.0601 \\
(\mathrm{n}=19)\end{array}$ & 0.0285 & 0.012 & 0.019 & 0.121 \\
\hline 115 & $\begin{array}{c}0.1305 \\
(\mathrm{n}=3)\end{array}$ & 0.0220 & $\begin{array}{c}0.0861 \\
(22)\end{array}$ & 0.0221 & $\begin{array}{l}0.0818 \\
(\mathrm{n}=21)\end{array}$ & 0.0371 & 0.010 & 0.050 & 0.308 \\
\hline 135 & $\begin{array}{c}0.1408 \\
(n=3)\end{array}$ & 0.0289 & $\begin{array}{c}0.0951 \\
(20)\end{array}$ & 0.0202 & $\begin{array}{l}0.1092 \\
(\mathrm{n}=19)\end{array}$ & 0.0438 & 0.014 & 0.232 & 0.431 \\
\hline 155 & $\begin{array}{c}0.1471 \\
(\mathrm{n}=3)\end{array}$ & 0.0179 & $\begin{array}{l}0.1082 \\
(n=16)\end{array}$ & 0.276 & $\begin{array}{l}0.1146 \\
(\mathrm{n}=15)\end{array}$ & 0.0501 & 0.044 & 0.260 & 0.477 \\
\hline 175 & $\begin{array}{c}0.2294 \\
(n=3)\end{array}$ & 0.0648 & $\begin{array}{l}0.1721 \\
(n=12)\end{array}$ & 0.0629 & $\begin{array}{c}0.1419 \\
(\mathrm{n}=7)\end{array}$ & 0.0719 & 0.112 & 0.138 & 0.205 \\
\hline
\end{tabular}


Table 16 - Kruskal-Wallis Test Results Comparing the Exfiltration Rate Values in Each Year, Control $19 \mathrm{H}$

\begin{tabular}{|c|c|c|c|c|c|c|c|c|c|}
\hline \multirow{3}{*}{$\begin{array}{l}\text { Water } \\
\text { Level } \\
(\text { cm) }\end{array}$} & \multicolumn{6}{|c|}{ Exfiltration Rates $(\mathrm{cm} / \mathrm{hr})$} & \multicolumn{3}{|c|}{ Comparison Between } \\
\hline & \multicolumn{2}{|c|}{2011} & \multicolumn{2}{|c|}{2012} & \multicolumn{2}{|c|}{2013} & \multirow{2}{*}{$\begin{array}{c}2011 \\
\& \\
2012 \\
P \\
\text { Value }\end{array}$} & \multirow{2}{*}{$\begin{array}{c}2011 \\
\& \\
2013 \\
P \\
\text { Value }\end{array}$} & \multirow{2}{*}{$\begin{array}{c}2012 \\
\& \\
2013 \\
P \\
\text { Value }\end{array}$} \\
\hline & Mean & St. Dev. & Mean & St. Dev. & Mean & St. Dev. & & & \\
\hline 15 & $\begin{array}{c}0.10330 \\
(n=3)\end{array}$ & 0.00810 & $\begin{array}{l}0.0807 \\
(\mathrm{n}=17)\end{array}$ & 0.0147 & $\begin{array}{l}0.0972 \\
(\mathrm{n}=21)\end{array}$ & 0.0322 & 0.023 & 0.407 & 0.025 \\
\hline 35 & $\begin{array}{c}0.1782 \\
(\mathrm{n}=3)\end{array}$ & 0.0200 & $\begin{array}{l}0.1341 \\
(n=26)\end{array}$ & 0.0269 & $\begin{array}{l}0.1964 \\
(\mathrm{n}=22)\end{array}$ & 0.0601 & 0.018 & 0.763 & $\mathbf{0 . 0 3 4}$ \\
\hline 55 & $\begin{array}{c}0.1987 \\
(\mathrm{n}=3)\end{array}$ & 0.0233 & $\begin{array}{l}0.1388 \\
(\mathrm{n}=32)\end{array}$ & 0.0213 & $\begin{array}{l}0.2327 \\
(\mathrm{n}=18)\end{array}$ & 0.0637 & 0.006 & 0.248 & 0.000 \\
\hline 75 & $\begin{array}{c}0.2577 \\
(n=3)\end{array}$ & 0.0354 & $\begin{array}{c}0.1673 \\
(26)\end{array}$ & 0.0303 & $\begin{array}{l}0.2882 \\
(n=14)\end{array}$ & 0.0493 & 0.005 & 0.257 & 0.000 \\
\hline 95 & $\begin{array}{c}0.2631 \\
(\mathrm{n}=3)\end{array}$ & 0.0630 & $\begin{array}{c}0.2261 \\
(18) \\
\end{array}$ & 0.0347 & $\begin{array}{c}0.3652 \\
(\mathrm{n}=9)\end{array}$ & 0.0825 & 0.228 & 0.079 & 0.000 \\
\hline 115 & $\begin{array}{c}0.3450 \\
(\mathrm{n}=3)\end{array}$ & 0.0367 & $\begin{array}{l}0.2879 \\
(\mathrm{n}=15)\end{array}$ & 0.0463 & $\begin{array}{l}0.455 \\
(\mathrm{n}=7)\end{array}$ & 0.0463 & 0.051 & 0.210 & 0.005 \\
\hline 135 & $\begin{array}{c}0.4069 \\
(\mathrm{n}=3)\end{array}$ & 0.0460 & $\begin{array}{l}0.3445 \\
(\mathrm{n}=11)\end{array}$ & 0.0378 & $\begin{array}{l}0.511 \\
(\mathrm{n}=3)\end{array}$ & 0.131 & 0.036 & 0.275 & 0.010 \\
\hline
\end{tabular}

The results from Table 15 show that control $19 \mathrm{G}$ has experienced significant decreases in exfiltration rates during the first year which is continued for lower depths of water in year 2013. On the other hand control $19 \mathrm{H}$ has shown significant increase in its exfiltration rates for year 2013 compared to year 2012. Overall the results indicate that while 19G has slower exfiltration rates in 2013 compared to 2011, especially for lower levels of water, control $19 \mathrm{H}$ has had restoration in its exfiltration performance and no significance difference between values from 2011 and 2013 is observed for this control.

Since the variations of calculated exfiltration rates suggest existence of some temperature dependency, the effect of temperature on exfiltration rates is first investigated. The calculated exfiltration rates versus the changes of temperature at the bottom of the 
trench, for both controls $19 \mathrm{G}$ and $19 \mathrm{H}$, are plotted with linear trend lines in Figures 52 and 53. Since the calculated exfiltration rates for different levels of water follow the same trend as each other, only one level is selected from each control. The selected levels, 95 centimeter for $19 \mathrm{G}$ and 75 centimeter for $19 \mathrm{H}$, are approximately located in mid-range of water level changes in their controls, have enough data points over the study period, and are located at depths which are unlikely to be affected by antecedent dry period conditions. The exfiltration rates versus the temperature are plotted separately for years 2012 and 2013, excluding 3 events from year 2011.

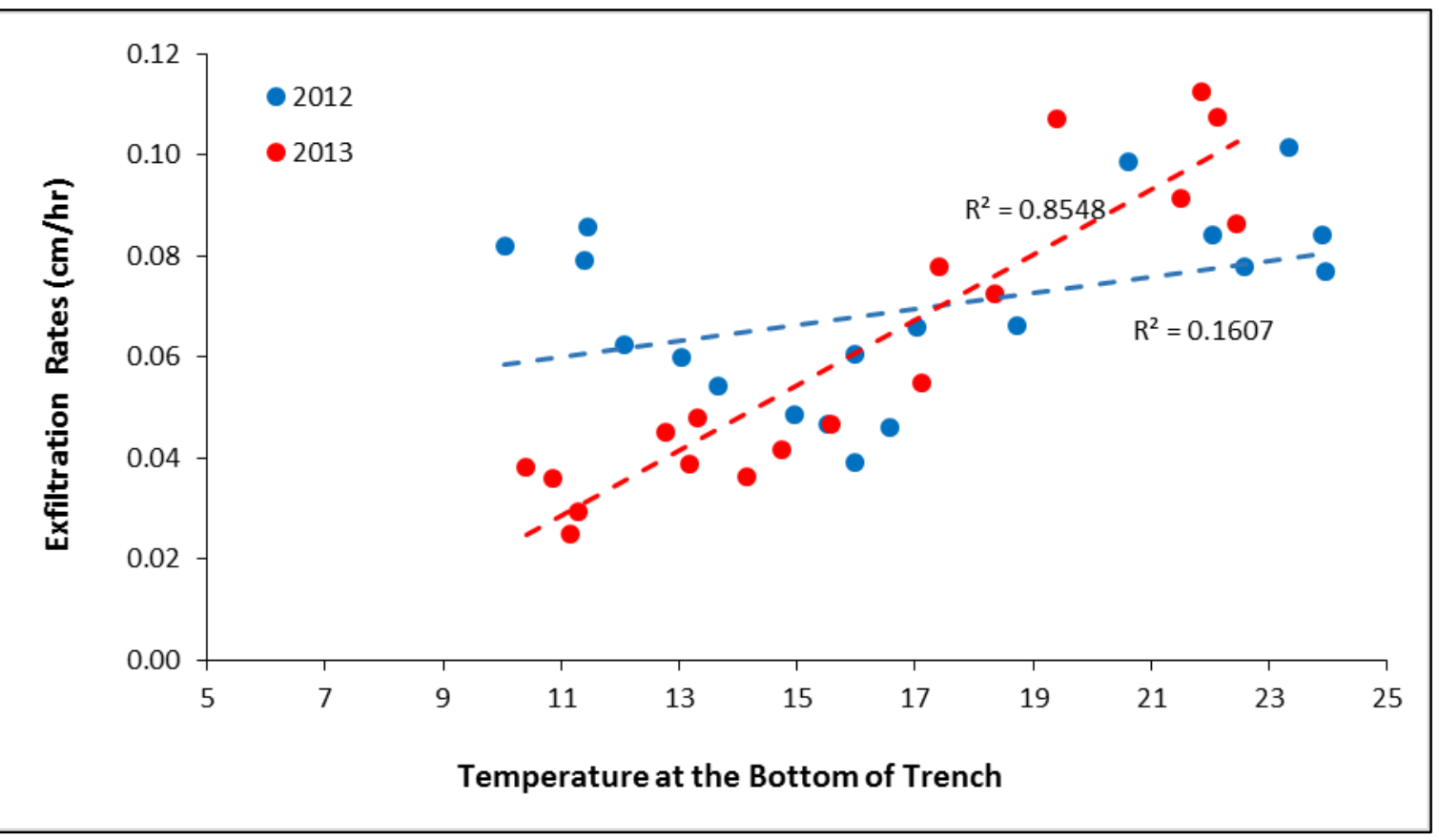

Figure 52 - Temperature Dependency of Exfiltration Rates versus Temperature, Control 19G 


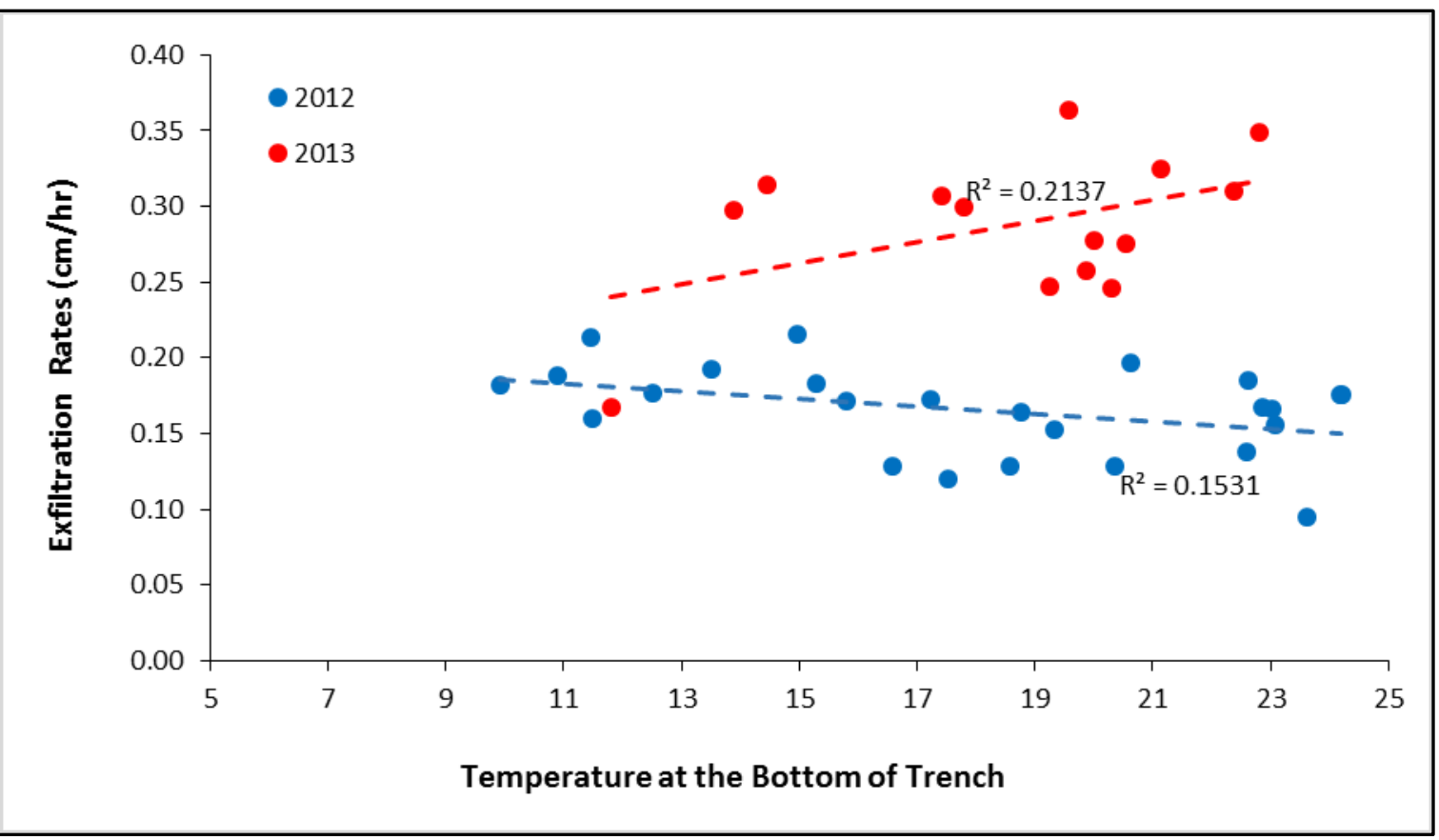

Figure 53 - Temperature Dependency of Exfiltration Rates versus Temperature, Control 19H

Figures 52 and 53 show that no linear relationship between temperature and calculated exfiltration rates can be observed for year 2012. In year 2013 a direct relationship can be observed between calculated exfiltration rates and temperature for control 19G and very hardly (low R-squared value) for control 19H as well. It appears that during the first year of their service lives both GI controls have experienced a decrease in their exfiltration rates regardless of changes in temperature values which has negatively affected their temperature dependency. This is also evident in Figures 48 and 49 which show that the increase in exfiltration rates of both GI controls during summer 2013 were greater than the one from summer 2012. This initial significant decrease is suspected to be due to depositing of sediments from runoff flow, attached solids from storage layer stones, and remaining debris from construction. 
In the next step the definition of hydraulic conductivity is used to further investigate the temperature dependency of exfiltration rates. Hydraulic conductivity values and exfiltration rates are both expressed by the same unit $\left(\mathrm{LT}^{-1}\right)$ but the concepts are different. Hydraulic conductivity, mostly used in Geotechnical engineering, is a term that defines the ability of a saturated soil medium to transmit water through its pore spaces. Darcy's law indicates that the velocity of water through a saturated soil medium is increased with an increase of the hydraulic gradient but the value of hydraulic conductivity is constant with respect to changes in hydraulic head (or gradient). On the other hand, infiltration/exfiltration rate of a soil layer, mostly used in soil science and agricultural applications, refers to the rate at which a soil medium is able to absorb water (from rainfall or irrigation). Infiltration/exfiltration rates are greater for unsaturated soil layers compared to saturated soils and are increased with an increase in hydraulic head. Although these two concepts are different, the hydraulic conductivity values and infiltration/exfiltration rates are related to each other as an increase in hydraulic conductivity value of a soil layer results in higher infiltration/exfiltration rate. In this study since the exfiltration rates are calculated for fixed interval levels of water, the effect of hydraulic head changes on exfiltration rates are equal for all calculated rates in that specific water level and therefor are ignored. The hydraulic conductivity is defined as (Hillel 1998):

$$
K=\frac{k \rho g}{\mu}
$$

Where:

$$
\begin{aligned}
& \mathrm{K}=\text { Hydraulic conductivity }\left[\mathrm{LT}^{-1}\right] \\
& \mathrm{k}=\text { Intrinsic permeability of soil layer }\left[\mathrm{L}^{2}\right]
\end{aligned}
$$




$$
\begin{aligned}
& \rho=\text { density of the fluid }\left[\mathrm{ML}^{-3}\right] \\
& \mathrm{g}=\text { gravimetric acceleration }\left[\mathrm{LT}^{-2}\right] \\
& \mu=\text { Dynamic viscosity of the fluid }\left[\mathrm{ML}^{-1} \mathrm{~T}^{-1}\right]
\end{aligned}
$$

Equation 4.3 shows that not only the hydraulic conductivity values are a property of soil (intrinsic permeability) but they are also dependent on the characteristics of the fluid, especially the density and dynamic viscosity of the fluid. The intrinsic permeability of soil layers depends on type of soil and most importantly the pose spaces within the soil fabric. The relationship between dynamic viscosity of the fluid and temperature can be defined with Vogel equation:

$$
\mu=e^{A+\frac{B}{C+T}}
$$

In which:

$\mu=$ Dynamic viscosity of the fluid $(\mathrm{Pa} . \mathrm{S}) \times 10^{-3}$

$\mathrm{T}=$ Fluid Temperature (Kelvin)

$\mathrm{A}=-3.7188$

$B=578.919$

$\mathrm{C}=-137.546$

Equation 4.3 can be rewritten as:

$$
K=k \frac{\rho g}{\mu}=k \times f
$$

Where:

$\mathrm{K}=$ Hydraulic conductivity $\left[\mathrm{LT}^{-1}\right]$

$\mathrm{k}=$ Intrinsic permeability of soil layer $\left[\mathrm{L}^{2}\right]$ 
$\mathrm{f}=\frac{\rho g}{\mu}=$ fluidity of water $\left[\mathrm{L}^{-1} \mathrm{~T}^{-1}\right]$

Since we are observing a limited range of temperatures throughout each year at the bottom of trenches (10 - 30 Celsius), we may assume constant density for the water and then the fluidity versus the temperature can be estimated as shown Figure 54.

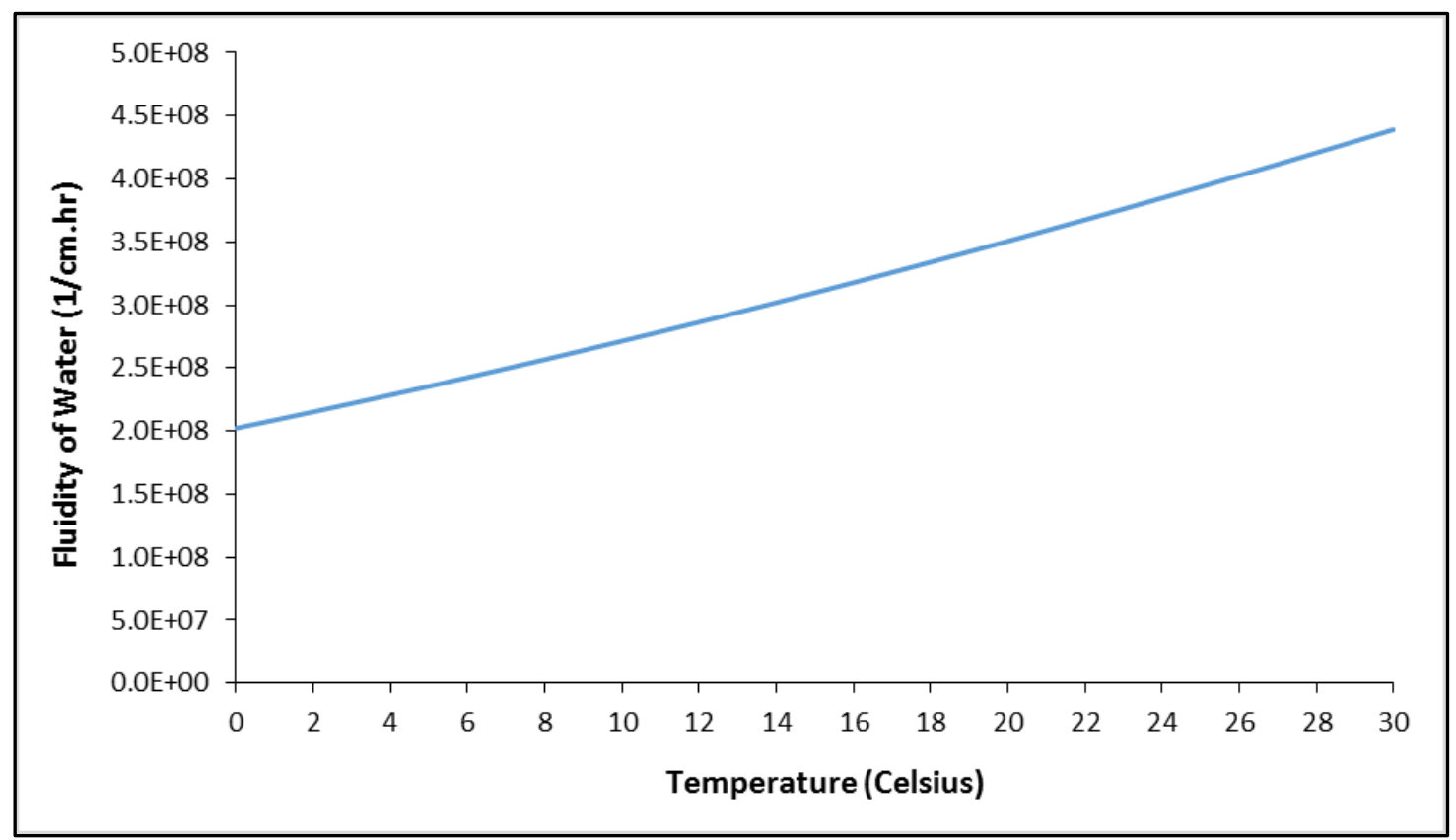

Figure 54 - Fluidity of Water versus Temperature

To further investigate if the temperature dependency of exfiltration rates in 2013 is caused by changes in water viscosity, a simple analysis based on equation 4.5 is performed. It must be noted that this analysis methodology is adopted from an effort conducted by Emerson (2008) to investigate the seasonal variations of three infiltration GI controls (Emerson 2008). In this analysis the average of temperature value is first calculated for data points in Figures 50 and 51 and for year 2013. By having the average temperature value, the corresponding average hydraulic conductivity (exfiltration rate) value can be estimated by use of the linear regression similar to the ones shown in Figures 50 and 51. 
Then using the calculated average hydraulic conductivity and the reference temperature value, an average value for intrinsic permeability of soils layers can be estimated. The results from these calculations are provided in Table 17.

Table 17 - Average Intrinsic Permeability Values Estimated by Considering Fluidity of Water, year 2013

\begin{tabular}{lcccc}
\hline GI Control ID & $\begin{array}{c}\text { Water } \\
\text { Level }\end{array}$ & $\begin{array}{c}\text { Average } \\
\text { Temperature } \\
\text { (Celsius) }\end{array}$ & $\begin{array}{c}\text { Average Hydraulic } \\
\text { Conductivity } \\
\text { (cm/hr) }\end{array}$ & $\begin{array}{c}\text { Estimated Average } \\
\text { Intrinsic } \\
\text { Permeability }\left(\mathbf{c m}^{2}\right)\end{array}$ \\
\hline \multirow{4}{*}{$\mathbf{1 9 G}$} & $95-\mathrm{cm}$ & 15.98 & 0.060 & $1.891 \mathrm{E}-10$ \\
\cline { 2 - 5 } & $115-\mathrm{cm}$ & 15.48 & 0.082 & $2.627 \mathrm{E}-10$ \\
\hline & $135-\mathrm{cm}$ & 16.63 & 0.113 & $3.496 \mathrm{E}-10$ \\
\hline \multirow{3}{*}{$\mathbf{1 9 H}$} & $155-\mathrm{cm}$ & 15.38 & 0.114 & $3.659 \mathrm{E}-10$ \\
\hline & $175-\mathrm{cm}$ & 14.30 & 0.142 & $4.678 \mathrm{E}-10$ \\
\hline & $15-\mathrm{cm}$ & 16.45 & 0.091 & $2.840 \mathrm{E}-10$ \\
\hline & $35-\mathrm{cm}$ & 17.01 & 0.197 & $6.045 \mathrm{E}-10$ \\
\hline & $55-\mathrm{cm}$ & 18.33 & 0.235 & $6.980 \mathrm{E}-10$ \\
\hline & $75-\mathrm{cm}$ & 18.66 & 0.288 & $8.486 \mathrm{E}-10$ \\
\hline
\end{tabular}

Since in this analysis the intrinsic permeability values are calculated by assuming exfiltration rates equal to the hydraulic conductivity values of existing soil layers, the resultant intrinsic permeability values are greater for higher levels of water. These values could only be representative of true intrinsic permeability values of underlying soil layer for very shallow depths of water, in which the effect of hydraulic gradient/head is minimal.

In the next step the calculated intrinsic permeability values from Table 17 are assumed constant with time and temperature for all data points in their level. Having the temperature values for each data point, the fluidity of water is calculated and then multiplied by the intrinsic permeability values to estimate hydraulic conductivity values. The resultant hydraulic conductivity values (corrected for fluidity of water) and the observed exfiltration rates are plotted versus the temperature and are shown in Figure 55. 


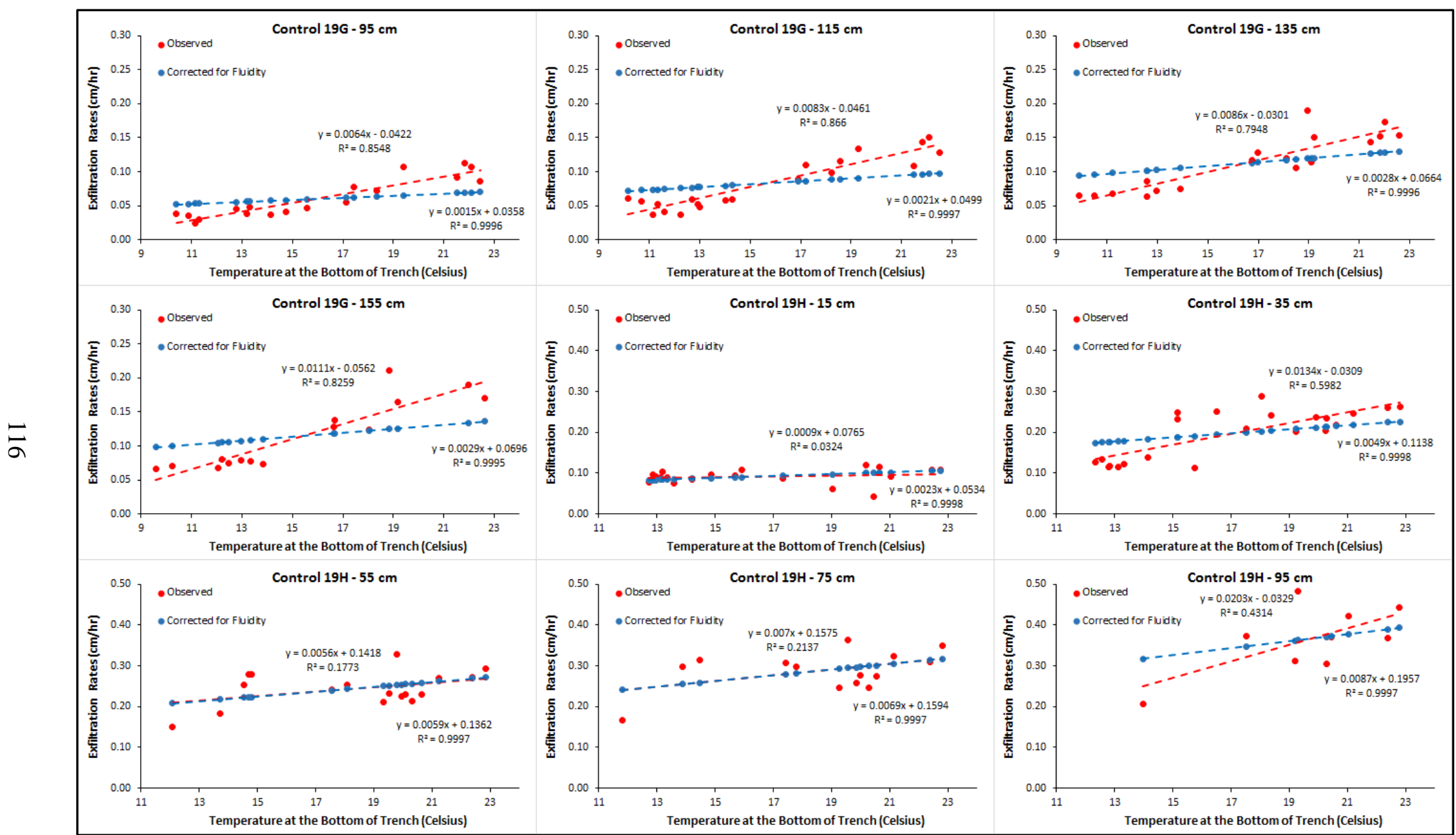

Figure 55 - Observed and Corrected for Fluidity of Exfiltration Rates for Year 2013, Controls 19G and 19H 
Results for Figure 55 show that the slopes between linear regressions of observed and estimated exfiltration rates versus temperature, are closer to each other for control $19 \mathrm{H}$ compared to control 19G. While these results indicate that some of the temperature dependency of exfiltration rates in control $19 \mathrm{H}$, if any, are caused by changes in viscosity of the water, involvement of another temperature dependent factor is suspected for control 19G. It must also be noted that the shown data points in Figure 55 are from year 2013 in which the exfiltration showed more temperature dependency. Hardly any relationship between exfiltration rates and measured temperatures was observed for year 2012 in any of the GI controls.

All calculated exfiltration rates from Figures 48 and 49 are divided by fluidity values of their corresponding temperatures to estimate their intrinsic permeability values. The resultant values are shown in Figures 56 and 57. As it can be seen in these figures, the intrinsic permeability values hardly show any temperature dependency for control $19 \mathrm{H}$. As for control 19G, the calculated intrinsic permeability values in year 2013 still show some temperature dependency, although with a less variability compared to exfiltration rates. Both controls have experienced a decrease in their intrinsic permeability values during the first year after their installation (2012), with some slight increases during summer time for control 19G.

To better evaluate the changes in calculated exfiltration rates with age, the calculated values are divided by their fluidity of their corresponding temperatures and the average of estimated intrinsic permeability values are presented separately for each year in Figures 58 and 59. The average temperature values at the bottom of the trench are also included in these Figures. 


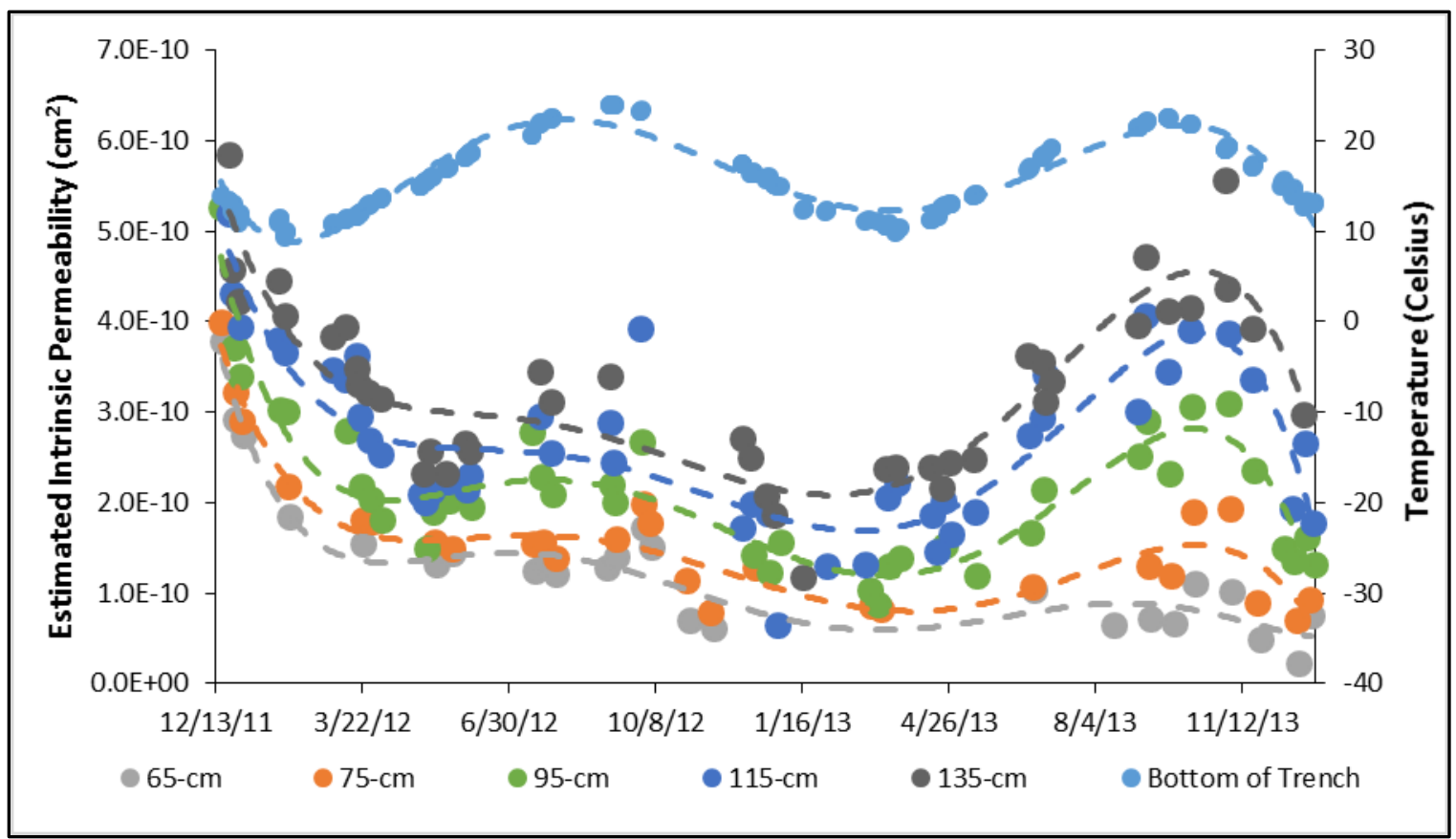

Figure 56 - Estimated Intrinsic Permeability for Control 19G

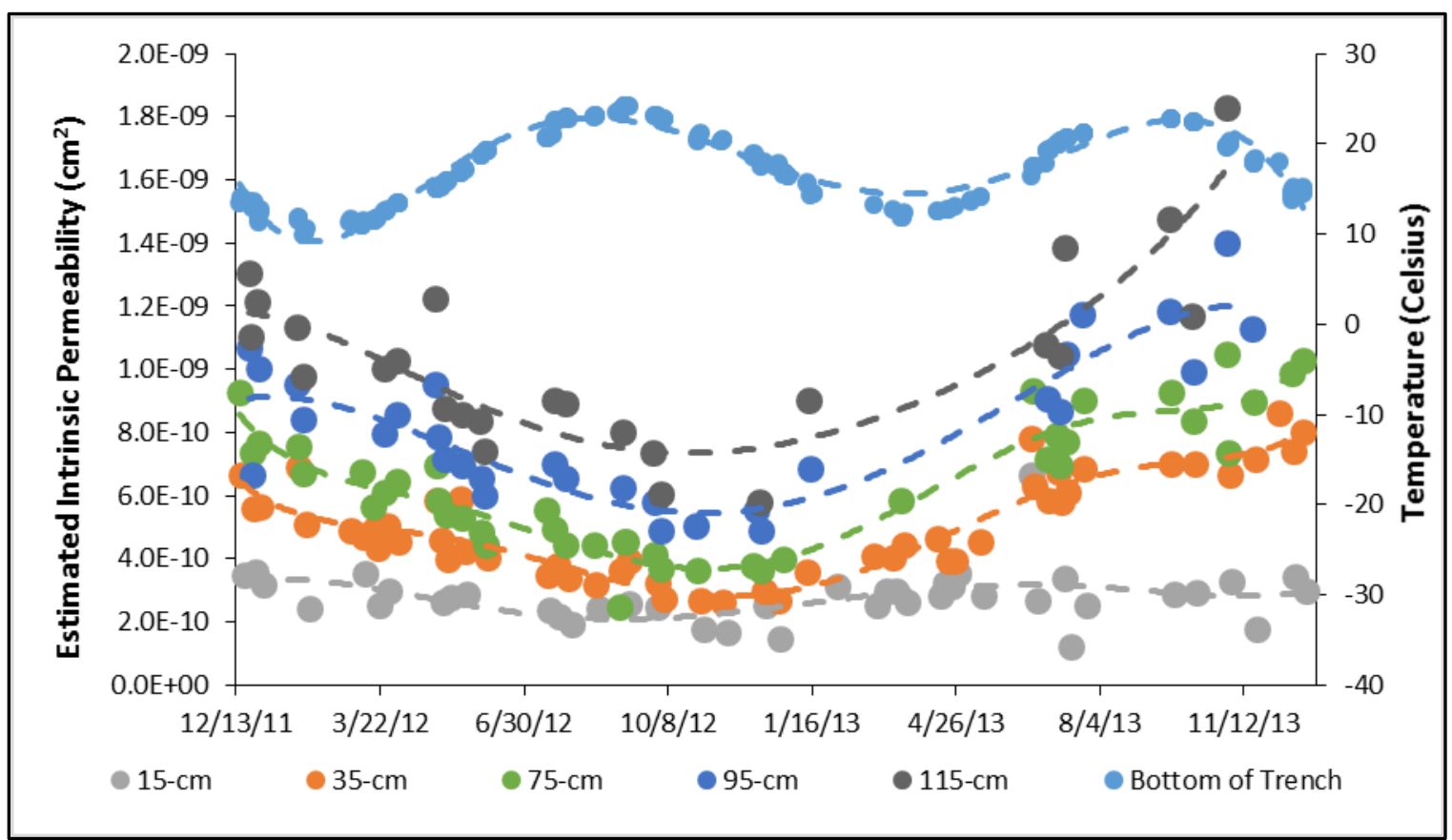

Figure 57- Estimated Intrinsic Permeability for Control $19 \mathrm{H}$ 


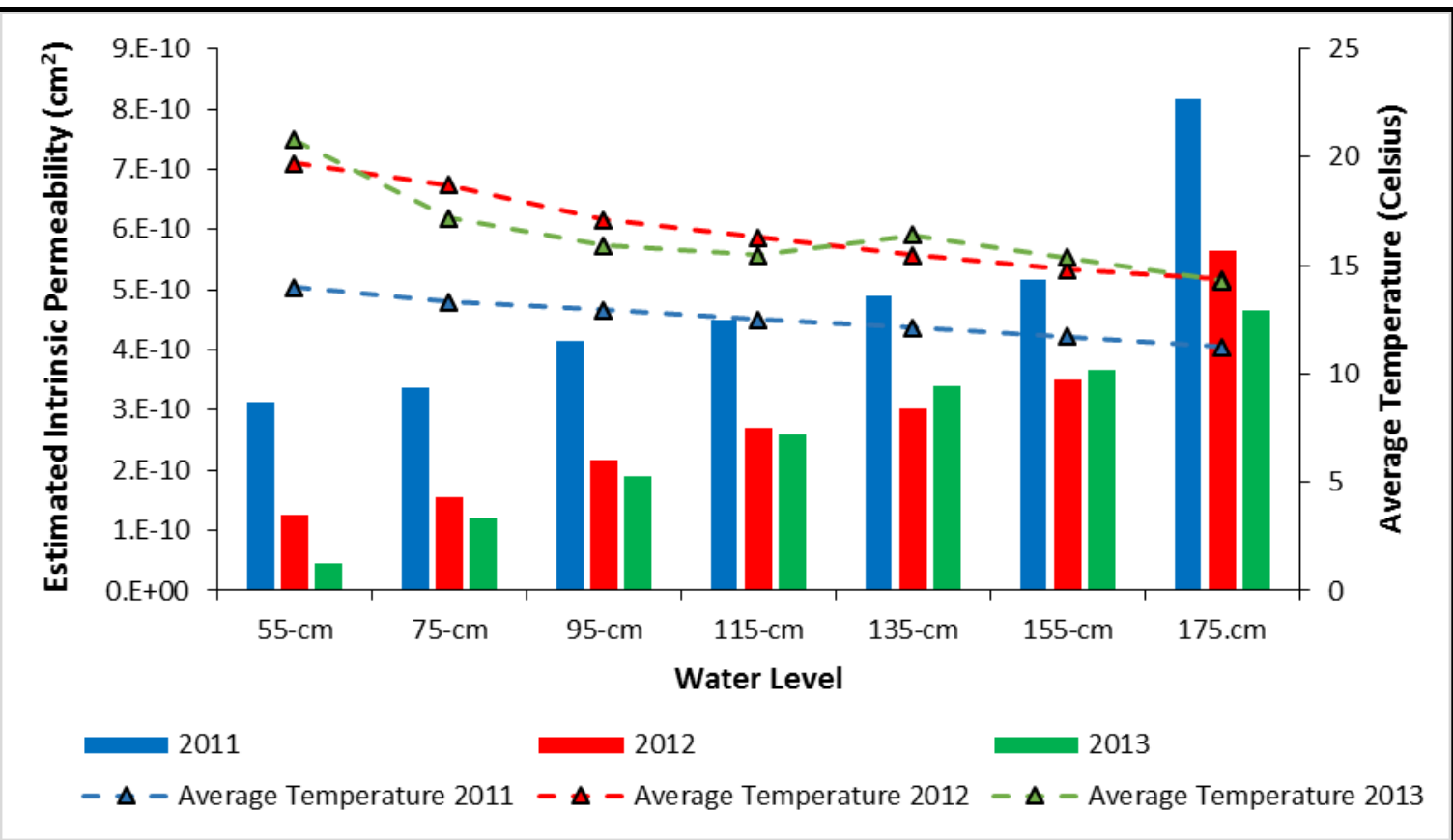

Figure 58 - Changes in Average Estimated Intrinsic Permeability Values with Age, Control 19G

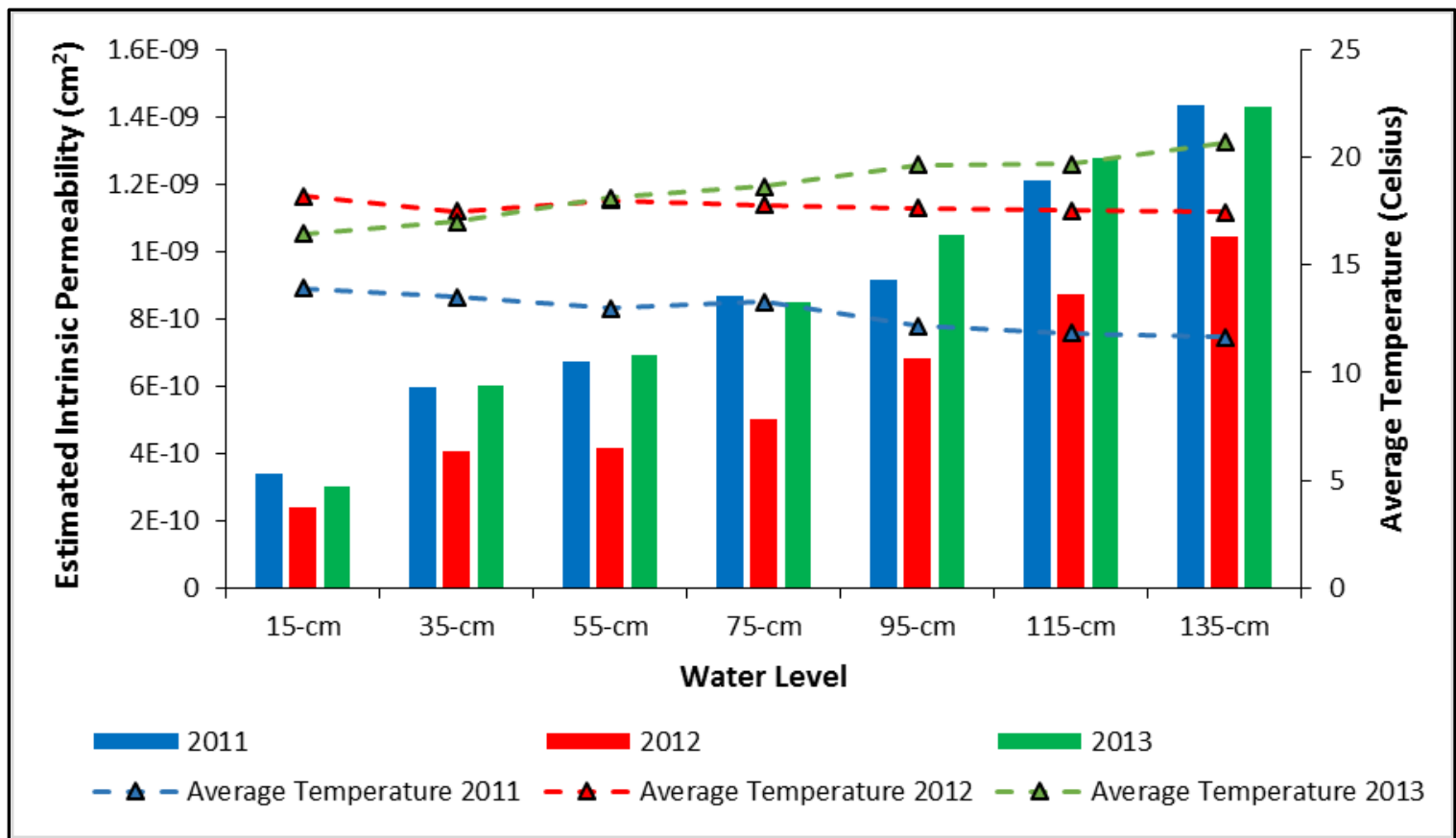

Figure 59 - Changes in Average Estimated Intrinsic Permeability Values with Age, Control 19H 
Since the estimated intrinsic permeability values are used in Figures 58 and 59, any possible temperature dependency observed in these two figures, is supposed to be caused by another factor besides the changes in viscosity of water. The first thing can be detected from these figures is that both GI controls have experienced significant decreases in their intrinsic permeability values (and exfiltration rates) in their second year (2012) of their service lives. Control 19H shows improvements in its exfiltration performance in 2013 compared to 2012, which doesn't appear to be temperature dependent. As shown on Figure 59 , the intrinsic permeability values for control $19 \mathrm{H}$ has been restored to their initial values in 2013. On the other hand changes in intrinsic permeability values of control $19 \mathrm{G}$ over years 2012 and 2013 seems likely to be mostly temperature dependent with no significant decreases in 2013 compared to 2012, with the exception of values for 55-cm water level.

The proposed explanation for observed changes in intrinsic permeability values hypothesizes a possible dependency of exfiltration performance on infiltration performance for both GI controls. Table 18 shows the information regarding the infiltration performances of controls $19 \mathrm{G}$ and $19 \mathrm{H}$. The numbers and their method of assessments are explained in Chapters 5 and 6.

Table 18 - Infiltration Performance of Controls 19G and 19H

\begin{tabular}{lcccc}
\hline Control ID & \multicolumn{2}{c}{$\begin{array}{c}\text { Volume Captured } \\
\text { (Thousand Gallons) }\end{array}$} & $\begin{array}{c}\text { Number of Successful Surface } \\
\text { Infiltration Restoration }\end{array}$ \\
\hline Year & 2012 & 2013 & 2012 & 2013 \\
\hline Control 19G & 180 & 170 & 1 & 2 \\
\hline Control 19H & 100 & 51 & 1 & 0 \\
\hline
\end{tabular}


Table 18 indicates that in 2013 control 19G has been able to capture approximately the same volume as it had in 2012. On the other hand in 2013 control 19H only captured half of the volume it had captured in the previous year. The changes in infiltration capacity of the GI controls is related to the number of successful maintenance treatment that they have had in each year which are reviewed in detail in chapter 6. During the surface maintenance treatments samples were collected from the sediments that clogged the surface of the GI controls. Laboratory test showed almost 30\% (by weight) of the clogging sediments were organic materials. Since these organic materials have low densities compared to non-organic fine materials, the volume percentage of the existing organic material in clogging sediments are possibly greater than $30 \%$.

The proposed hypothesis is that with each rainfall event some of these sediments are washed into the GI controls and are eventually deposited at the bottom of the trench resulting in decreased exfiltration rates. It is suspected that most of the sediments that were deposited with the first rainfall events came from the attached solids of storage layer stones (see Tables 5 and 6) and the remaining debris from the construction and together they caused rapid decreases in exfiltration performance in early 2012. On the other hand the source of other sediments that were deposited later in 2012 and 2013 was stormwater runoff that as explained are suspected of having significant organic materials. The organic materials decompose over time and the rate of their decomposition depends on various factors such as temperature, light, and available oxygen.

In the case of control 19G, in which the volume captured in second and third years were almost equal, temperature is possibly the dominant factor effecting the rate of decomposition of accumulated organic material inside the GI control. Since higher values 
of temperature quickens the rate of decomposition of organic materials, restoration of estimated intrinsic permeability values is observed during summer time in control 19G. On the other hand the decrease in captured volume by control $19 \mathrm{H}$ probably increases the ratio of organic materials decomposed to the new organic materials brought into the GI control, which results in full restoration of its exfiltration performance. Also the clogging material formed at surface of control 19H through year 2013 probably further decreased the rate of organic material washed into GI control by acting as a filter. Since less organic material were existent is control $19 \mathrm{H}$, the temperature dependency of their decomposition rate is hardly observed.

To confirm the proposed hypothesis with more confidence, monitoring the changes in exfiltration rates and even conducting several laboratory studies to model the accumulation and decomposition of organic material with temperature and its effects on infiltration rates of underlying soil layers is suggested for further research. 


\section{MODELING THE WATER LEVEL INSIDE GI CONTROLS}

To effectively monitor the hydrological performance of controls $19 \mathrm{G}$ and $19 \mathrm{H}$, spreadsheet modeling techniques were utilized. The spreadsheet modeling allows continuous calculations to predict the changes of water level inside the GI Controls for fixed segments of time. This modelling technique is based on the assumption that volume of water inside the control is continuously affected by the inflow and outflow volumes for each segment of time. This can be expressed as:

$$
\text { (Volume of Water) } t=\mathrm{i}=(\text { Volume of Water })_{\mathrm{t}=\mathrm{i}-1}+(\text { Inflow }) \mathrm{t=i}-(\text { Outflow }) \mathrm{t}=\mathrm{i}
$$

By having the volume of stormwater inside the GI control and utilizing the dimensions of GI control and porosity of storage media layers, the water level can be calculated. The inflow volumes are calculated by using the general form of equation used in rational method. The rational method estimates the peak rates of runoff for a catchment area with this equation:

\section{$\mathrm{Q}=\mathrm{CIA} \quad(5.1)$}

Where:

Q: the peak discharge rate (cfs)

C: runoff coefficient (dimensionless)

I: rainfall intensity (in/hr)

A: catchment area (acre) 
The rational method uses the following assumptions (Pazwash 2011):

(a) Runoff is proportional to the rainfall intensity

(b) Abstractions and losses linearly vary with rainfall and are incorporated in the runoff coefficient.

(c) The duration of storm event must be equal to or longer than time of concentration catchment area.

The runoff coefficient in the rational method varies from less than 0.1 (for lawns and sandy soils) to 0.95 (for paved surfaces in downtown areas). When there are multiple types of surfaces in the catchment area, a weighted estimate of $\mathrm{C}$ is calculated and used in the equation (Pazwash 2011). For fixed durations of rainfall the rational method equation can be used to estimate the volume of runoff as follows:

$$
\mathrm{V}=\mathrm{Q} / \mathrm{T}=\mathrm{C}(\mathrm{I} / \mathrm{T}) \mathrm{A}=\mathrm{CDA}(5.2)
$$

Where:

$\mathrm{V}$ : runoff volume (cf)

$\mathrm{T}$ : fixed duration of rainfall (hr)

D: rainfall depth for fixed duration of rainfall (inches)

This formula is used to calculate the inflow volumes into each GI control. Since the study area is located in a downtown urban area, the runoff coefficient is expected to be between 0.9 and 1. For simplicity the runoff coefficient is assumed to be equal to 1 and any errors that might be introduced because of this assumption is corrected in the calibration of the model which will be explained later. 
Since both of the GI controls had relatively small catchment areas (less than 1 acre) the time of concentrations were expected to be minimal. It was noticed that for most rainfall events it took almost (or less than) five minutes for the pressure transducers to response to the onset of rainfall event, therefore the models are developed for 5-minute intervals. Because of the limitations that this assumption might have caused, modelling results from major events are only used in assessing the hydrological performances of controls $19 \mathrm{G}$ and $19 \mathrm{H}$.

Calculating the outflow volumes was more challenging. Figures 32 and 33 show that recession of captured water level inside the control slows as water level decreases. This indicates that exfiltration rates from the GI controls are greater for higher levels of water. This is not unexpected since the increase of hydraulic head increases the flow of water through soil layers and therefor higher exfiltration rates are observed. Figure 60 shows this process. The exfiltration process shown in Figure 60 can be modeled in two different ways: first assuming one exfiltration rate for the entire GI control which varies with depth of water level, and second assuming different exfiltration rates for different segments of GI control which are constant, see Figure 61. In this study both techniques are used and different models are developed for each GI control. 


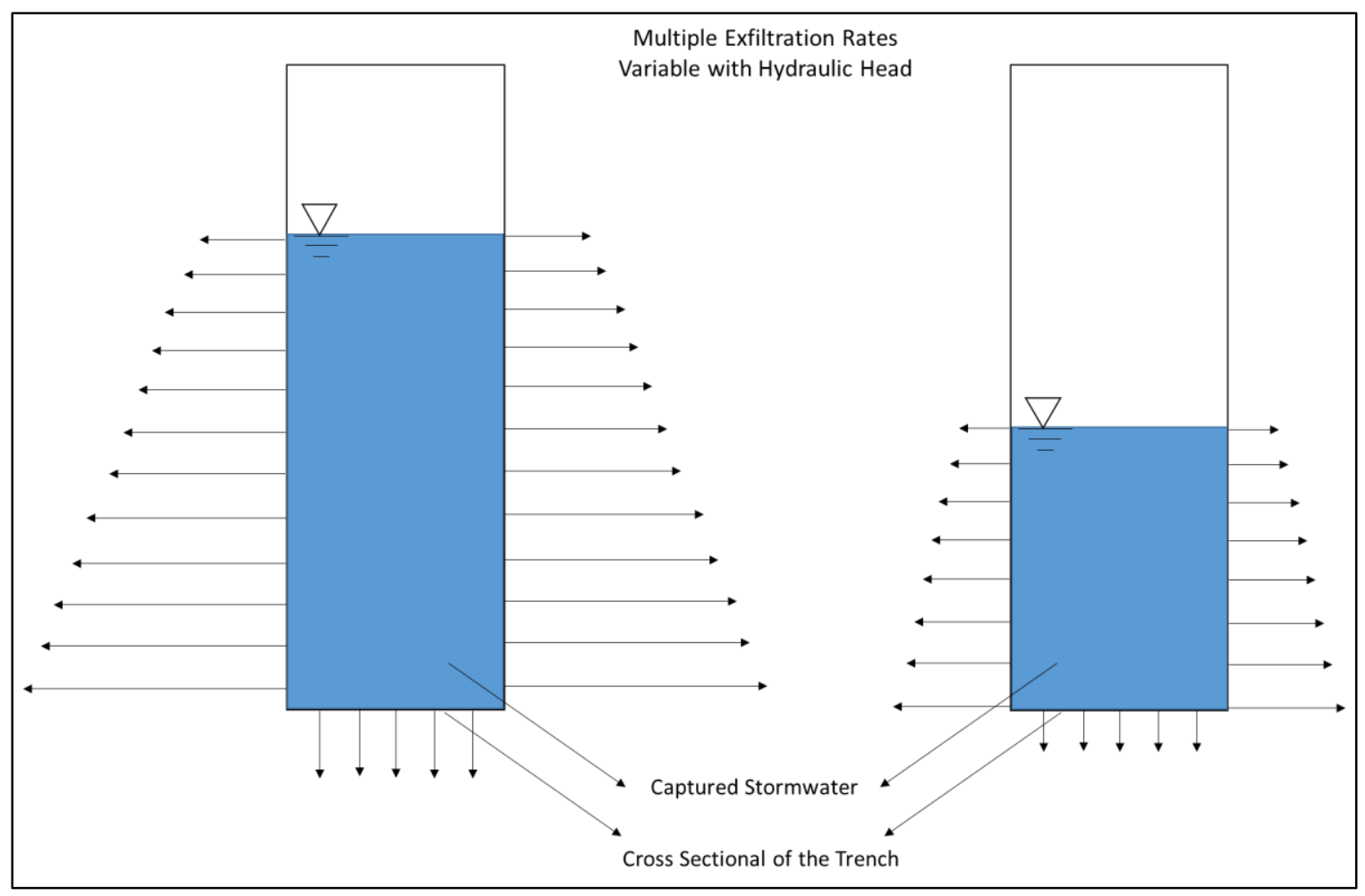

Figure 60 - Actual Exfiltration Performance from GI Controls

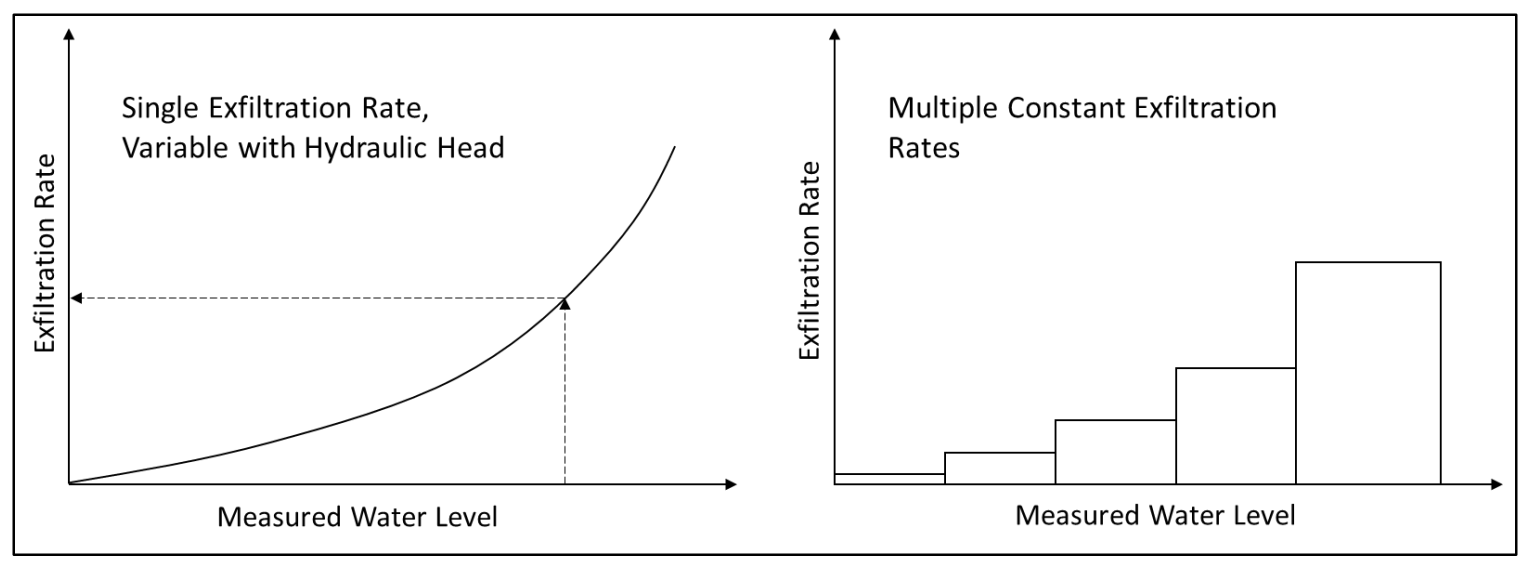

Figure 61 - Modeling Exfiltration Performance

By using the first technique (shown on left Figure 61), the average of observed exfiltration rates for the initial rainfall events are used for estimating the outflow volumes and then by adjusting the catchment area the model is calibrated for these events. By using the second technique, the inflow volumes are calculated by estimated values from Table 2 
and the model is calibrated by adjusting assumed exfiltration rates for different segments of GI control. These two different techniques have advantages over each other. The first technique better predicts the outflow volumes, since it is using the actual observed exfiltration rates, and also provides estimation of actual drainage areas for each GI control. The second technique is probably less reliable in estimating the outflow and inflow volumes but gives better understanding of exfiltration process and specific roles of different segments of GI controls which are contributing to the exfiltration process. Table 19 summarizes the characteristics and benefits of each modeling method. Both modeling techniques are explained in more detail in following sections.

Table 19 - Characteristics of Used Modeling Methods

\begin{tabular}{lll}
\hline & \multicolumn{1}{c}{ Technique 1 } & \multicolumn{1}{c}{ Technique 2 } \\
\hline Base assumption & $\begin{array}{l}\text { One exfiltration rate for entire GI } \\
\text { control, varying with water level }\end{array}$ & $\begin{array}{l}\text { Constant exfiltration rates for } \\
\text { different segments of GI control }\end{array}$ \\
\hline $\begin{array}{l}\text { Parameter adjusted } \\
\text { for calibration of } \\
\text { the model }\end{array}$ & Drainage area of the GI control & Exfiltration rate values \\
\hline $\begin{array}{l}\text { Benefits of the } \\
\text { selected method }\end{array}$ & $\begin{array}{l}\text { Provides better estimates of inflow } \\
\text { and outflow volumes, and } \\
\text { impermeable drainage area for the } \\
\text { GI control }\end{array}$ & $\begin{array}{l}\text { Provides better understanding of } \\
\text { exfiltration process and roles of } \\
\text { contributing segments of the GI } \\
\text { control }\end{array}$ \\
\hline
\end{tabular}

\subsection{Modeling Technique 1: One Variable Exfiltration Rate}

As previously explained, in this method, one varying exfiltration rate with water level is assumed for the entire GI control. The exfiltration rates used in the model are estimated by averaging the calculated exfiltration rates from the initial four events following the first event (event 2 through 5). The first rainfall event is intentionally excluded from both modeling methods because after this event substantial decrease of exfiltration rates were observed. This is suspected to be because of sediments from construction materials 
and storage layer stones getting washed into the GI control with the first rain event and resulting in decrease of exfiltration performance. By adjusting the drainage area, the model is calibrated for the seven rainfall events following the first rainfall event. During these events the surface clogging had not reached the downgradient side on both GI controls and no decrease in infiltration capacity was expected, which was desirable for calibrating the model. Figure 62 shows the observed and modeled water levels for control 19G.

Adjusting the drainage ratio for Control $19 \mathrm{H}$ was more challenging than it was for control 19G. It was noticed that for the rainfalls with higher intensities, more runoff volume was captured by control $19 \mathrm{H}$ and subsequently less captured runoff volumes were observed for rainfall events with lower intensities. Field visits during the rainfall events showed that a small alley, perpendicularly located upgradient of control $19 \mathrm{H}$, contributed to runoff volumes during more intense rain events. The surface of this alley, which was paved with bricks, was considered historic and therefor never repaved with asphalt. There was a catch basin at the center and downgradient of the alley but during intense rainfall events some stormwater runoff bypassed the catch basin and flowed toward control $19 \mathrm{H}$, resulting in captured runoff volumes greater than anticipated. 


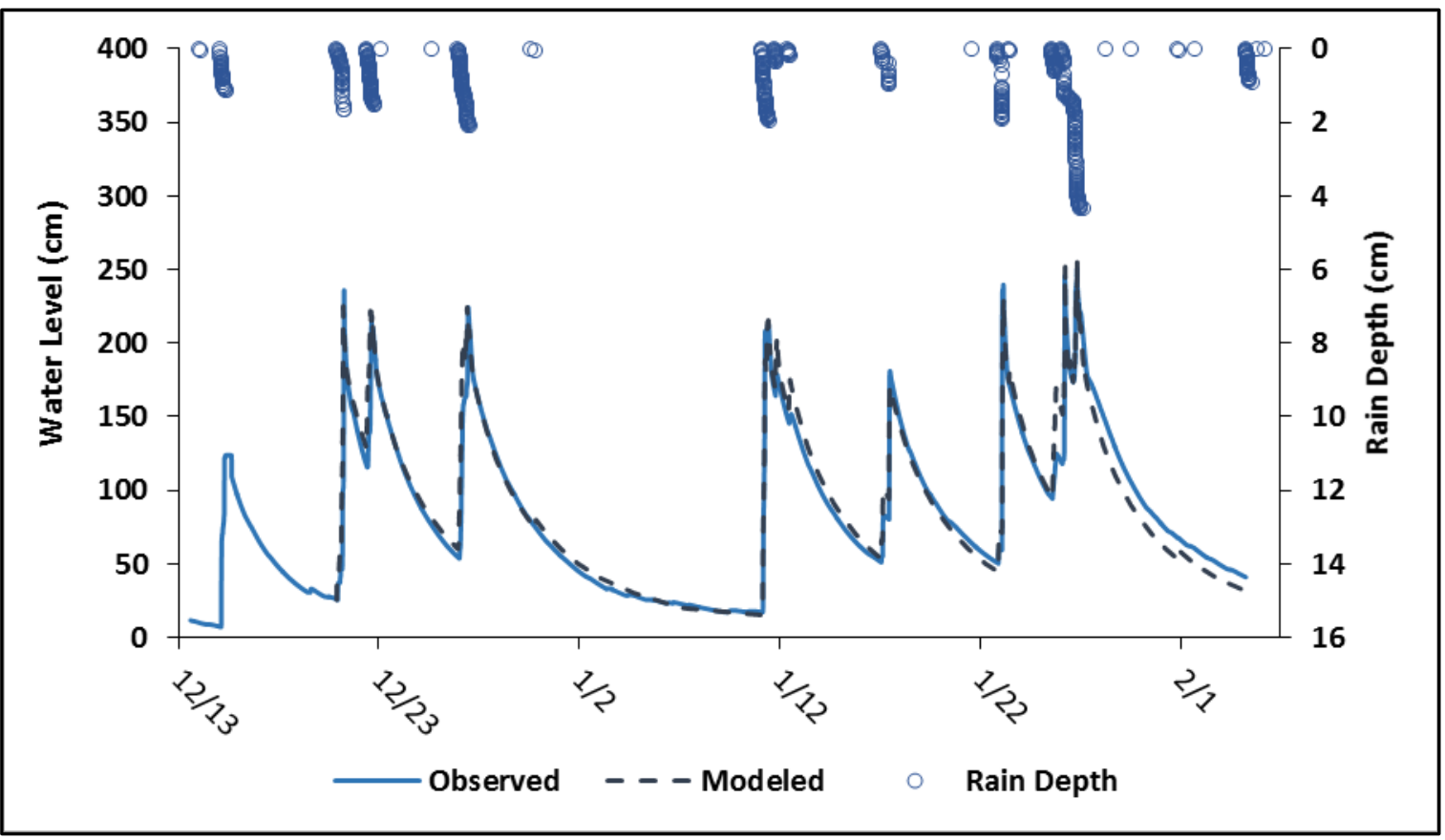

Figure 62 - Observed and Modeled Water Levels based on Technique 1, Control 19G

To provide a better evaluation of inflow volumes into control $19 \mathrm{H}$, two different values for drainage areas were estimated. The model uses one of these drainage area values based on the characteristics of rainfall events. The greater value is used for rainfall events that have been either greater than 1 (inch) or had a maximum 15-min rainfall intensity greater than 0.4 (in/hr) or a combination of both. With this definition, the third, fourth, and sixth rainfall events from the calibration period are considered small with low intensity rainfall events and the rest are characterized into high intensity rainfall events. As mentioned earlier the first rainfall event immediately after installation of controls $19 \mathrm{G}$ and $19 \mathrm{H}$ is excluded from calibration period events. Figure 63 shows the observed and modeled water levels for control $19 \mathrm{H}$. 


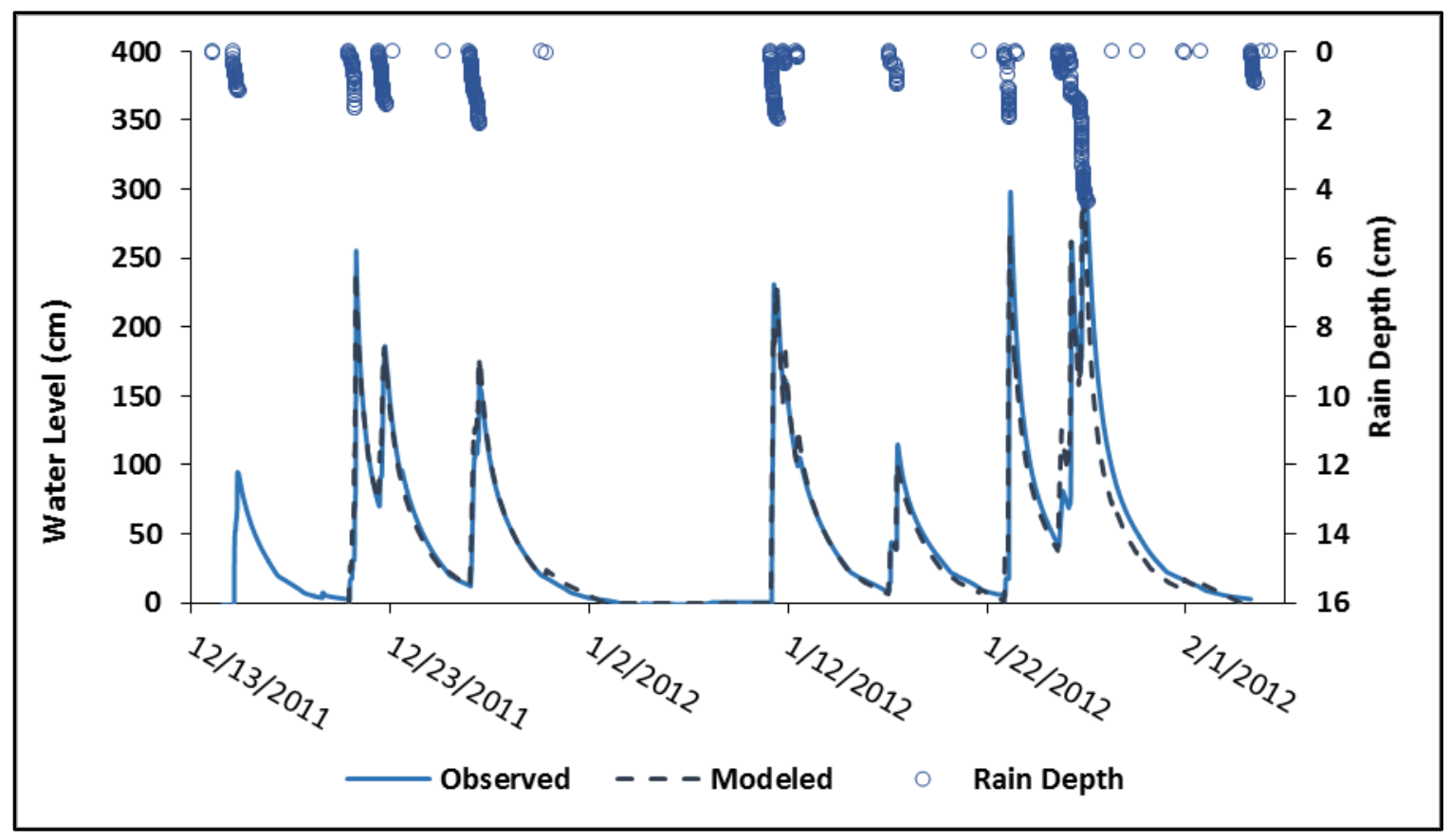

Figure 63 - Observed and Modeled Water Levels based on Technique 1, Control 19H

\subsection{Modeling Technique 2: Multiple Constant Exfiltration Rates}

In this technique the drainage values from Table 2 is used for calculating the inflow volumes. A percentage of impermeable draiange areas in Table 2, calcuated and used by URS Corp. during the design of controls $19 \mathrm{G}$ and $19 \mathrm{H}$, were rooftop areas that were supposed to contribute to the inflow volumes after disconnecting the downspouts from the exsiting combined sewer system. Since the downspout disconnections had not started until mid-2013 and also to account for variations in runoff coefficients for different surfaces of drainage areas of controls $19 \mathrm{G}$ and $19 \mathrm{H}$, the impermeable surfaces from Table 2 are decreased by the pecentage of connected roofop area. The URS Corp stated that these values to be $40 \%$ and $20 \%$ for controls $19 \mathrm{G}$ and $19 \mathrm{H}$, respectively. It must be noted that no excess in runoff volume is expected even after the downspouts are disconnected, because the stomwater runoff from these downspouts are mostly directed into lawns and vegetaed areas. 
To calculate the outflow volumes with this technique, the area inside each GI control was divided into ten different segments: one for the bottom of the trench, one for all areas (shoulders and lateral) in the storage gallery, and eight for the lateral area inside the trench. Segmenting of the lateral area started at the bottom with 30 centimeter increments and the last one covered all lateral areas higher than 210 centimeter. Eventually the outflow volume is calaculated by assuming constant exfiltration rates for the discrete soil layers and multiplying these rates by the wetted perimeter. By having the inflow and outflow volumes the water level inside the GI control can be calcualted. The model is then calibrated for the same rainfall events as technique 1 and by adjusting the assumed exfiltration rate values. Figures 64 and 65 show the modeled and observed water levels for controls 19G and 19H.

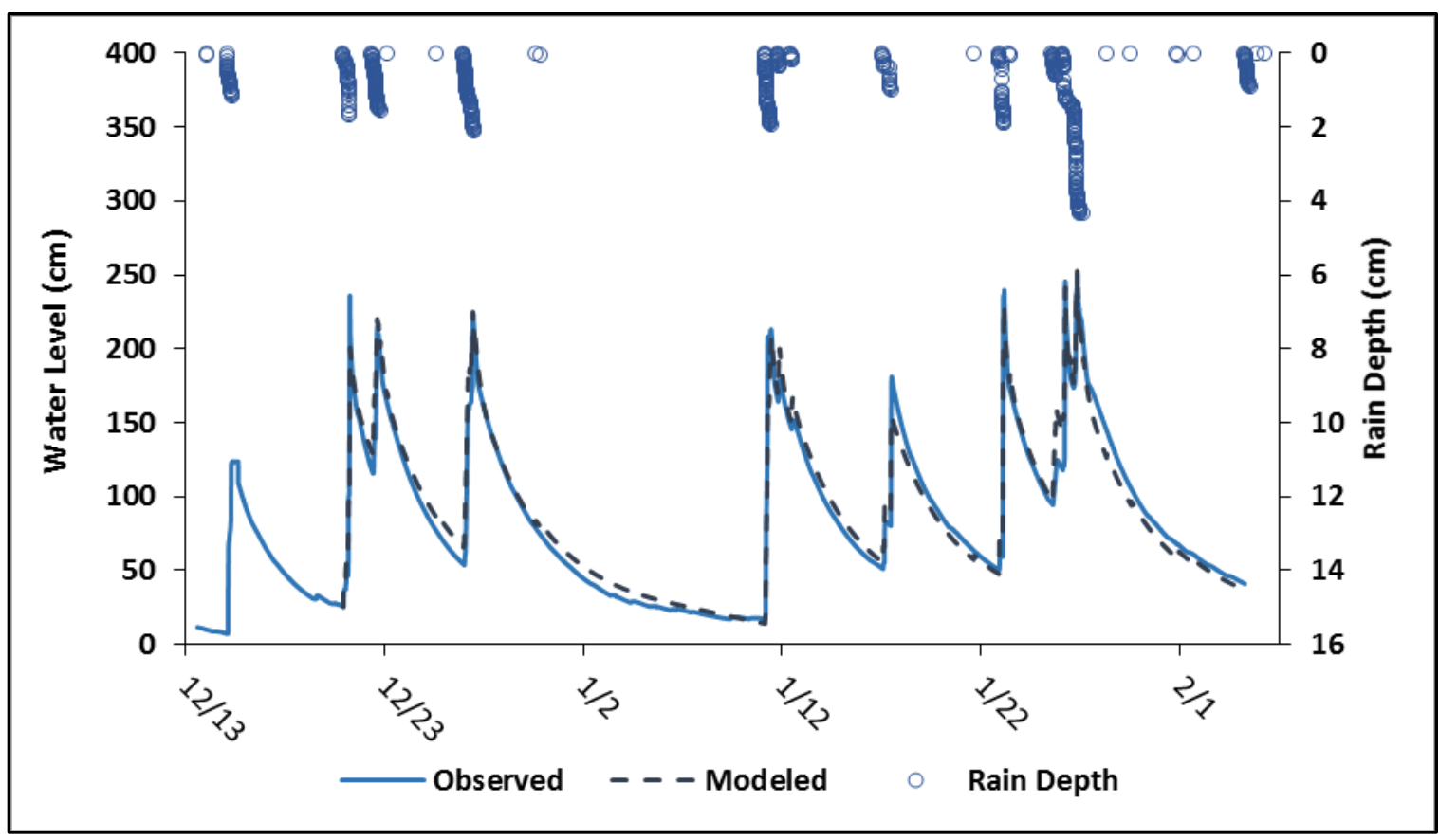

Figure 64 - Observed and Modeled Water Levels based on Technique 2, Control 19G 


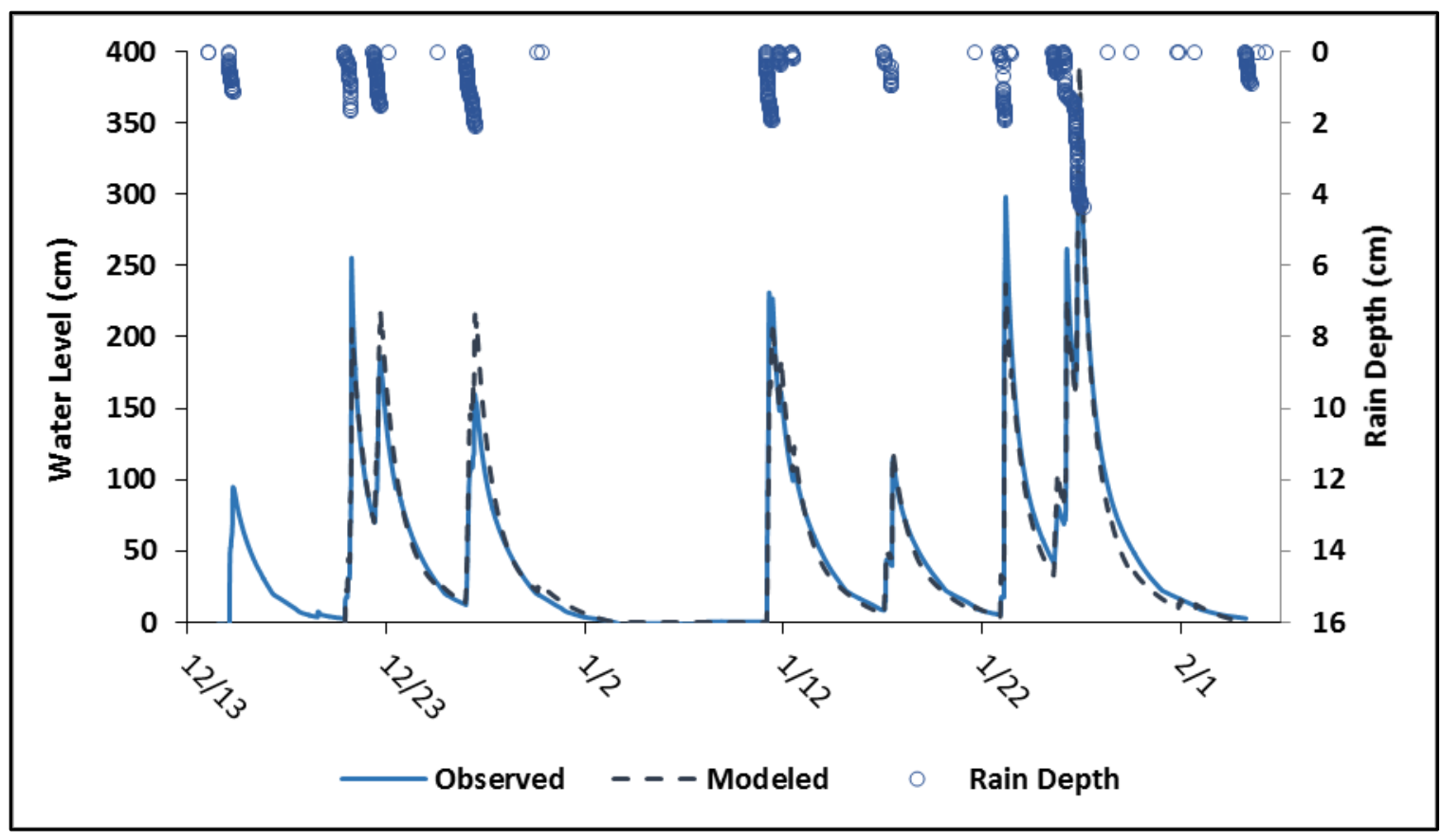

Figure 65 - Observed and Modeled Water Levels based on Technique 2, Control 19H

It must be noted that by using this method developing two models for control $19 \mathrm{H}$ wasn't practical since changing the drainage areas would have required readjusting the assumed exfiltration rate values and recalibration of the model. The second recalibrated model would have had different drainage areas and exfiltration rates than the first model and combining their results, similar to the proposed method suggested for Technique 1, wouldn't have been valid.

To compare the accuracies of developed models based the proposed techniques, the R-squared values are calculated for each developed model. The calculated R-squared values and effective impermeable drainage areas for each control are provided in Table 20. 
Table 20 - R-Squared and Drainage Areas from both Modeling Techniques

\begin{tabular}{lcccc}
\hline Modeling Technique & $\begin{array}{c}\text { Technique 1 } \\
\text { (One variable exfiltration } \\
\text { rate) }\end{array}$ & \multicolumn{2}{c}{$\begin{array}{c}\text { Technique 2 } \\
\text { (Multiple constant exfiltration } \\
\text { rates) }\end{array}$} \\
\hline Control ID & $19 \mathrm{G}$ & $19 \mathrm{H}$ & $19 \mathrm{G}$ & $19 \mathrm{H}$ \\
\hline R-Squared Value & 0.96 & 0.97 & 0.97 & 0.96 \\
\hline $\begin{array}{l}\text { Impermeable } \\
\text { Drainage Area } \\
\text { (acre) }\end{array}$ & 0.29 & $0.108-0.196$ & $0.242^{*}$ & $0.132^{*}$ \\
$\begin{array}{l}\text { *Values derived from Table 2 which are used during design of controls 19G and 19H, provided by } \\
\text { URS Corp. }\end{array}$
\end{tabular}

Although both techniques were able to provide satisfying R-Squared values (close to 1), calibrating the model developed from the second method was found to be more challenging and time consuming compared to the one developed by the first method, mostly because it required adjusting multiple values (constant exfiltration rates) simultaneously.

The developed models from the second technique provided more insight into the exfiltration performance of the controls $19 \mathrm{G}$ and $19 \mathrm{H}$. The calibrated models suggest that during the calibration period, most of captured stormwater exfiltrates through lateral areas of the trenches, see Figure 66. This seems reasonable because of the large ratios of trenches' sidewall areas to the bottom areas, which were approximately 10:1 for both GI controls. Infiltration measurements during construction also had indicated existence of soils layers with low hydraulic conductivity values at the bottom of the trenches, see Table 4 . The model also predicted that a small percentage of stormwater has exfiltrated from the storage gallery in control 19H and none from control 19G's. This was also anticipated since a comparison between infiltration rates of controls $19 \mathrm{G}$ and $19 \mathrm{H}$ indicates that control $19 \mathrm{H}$ fills up quicker than control 19G, see Figure 39. 


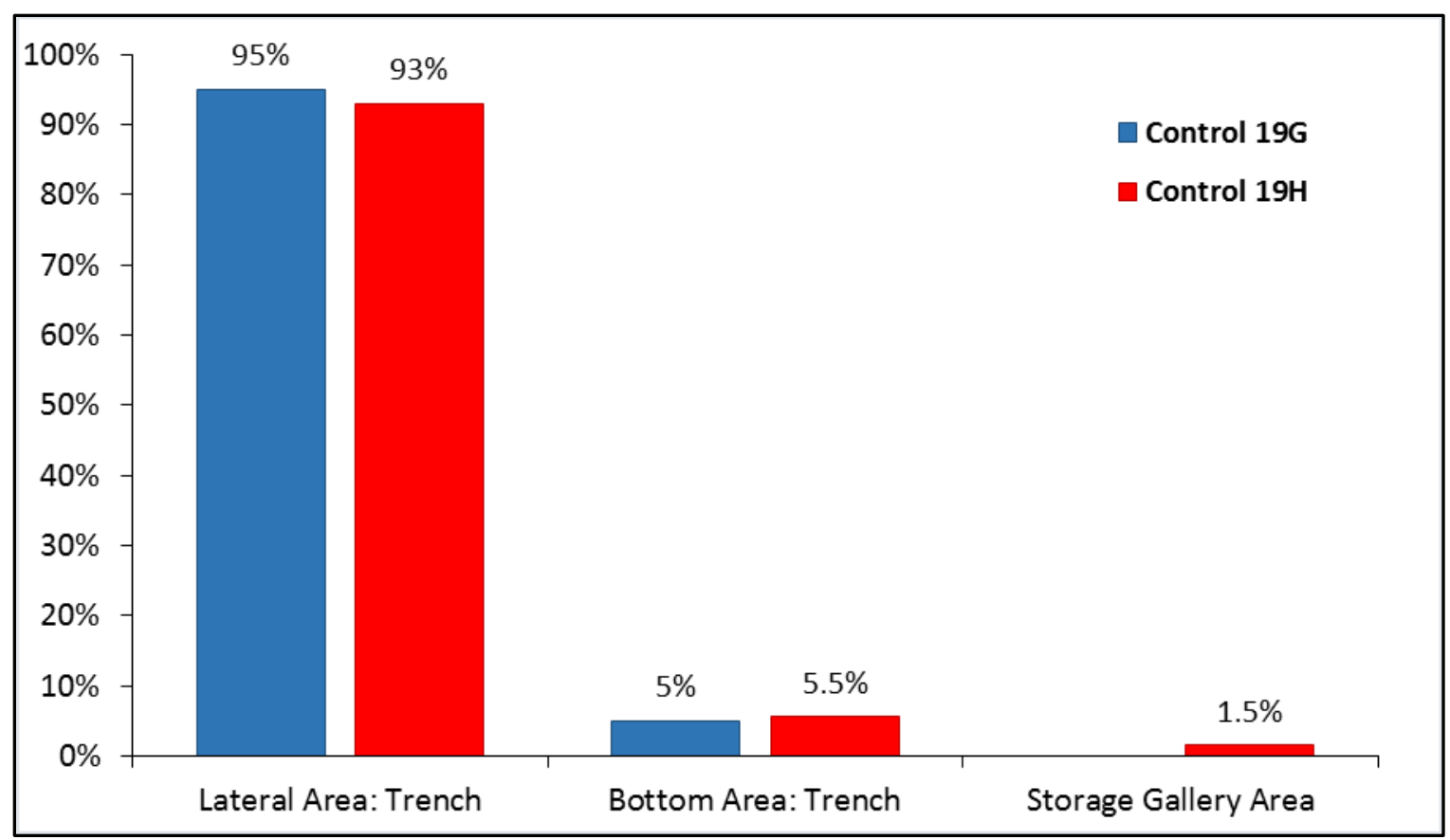

Figure 66 - Suspected Exfiltration Process from Modeling Technique 2, Controls 19G and 19H

\subsection{Modeling Results}

After calibration of both developed models they were used to assess the changes in hydrological performances of controls $19 \mathrm{G}$ and $19 \mathrm{H}$ over the course of this study. This was done by separating the "rises" and "drops" of water level inside the GI controls into independent events. For this analysis only rainfall events greater than 0.5 inches $(1.127$ centimeters) are selected and then the observed rises and drops of water levels associated with each event are used to assess changes in infiltration capacity and exfiltration performance, respectively. To do this before the start of each rainfall event the model is reset (by assuming a water level for the model equal to the observed water level) and then the peak values of observed and modeled water levels during the course of rainfall event are identified. At the end of the rainfall event the model is reset once again and this time the minimum levels of observed and modeled level are identified, see Figure 67. Having these 
maximum and minimum values after each rainfall event enable measuring the infiltration capacity and exfiltration performance with the following formulas:

$$
\begin{gathered}
\text { Infiltration Capacity }=\frac{\text { Observed Rise }}{\text { Predicted Rise }} \times 100 \\
\text { Exfiltration Performance }=\frac{\text { Observed Drop }}{\text { Predicted Drop }} \times 100
\end{gathered}
$$

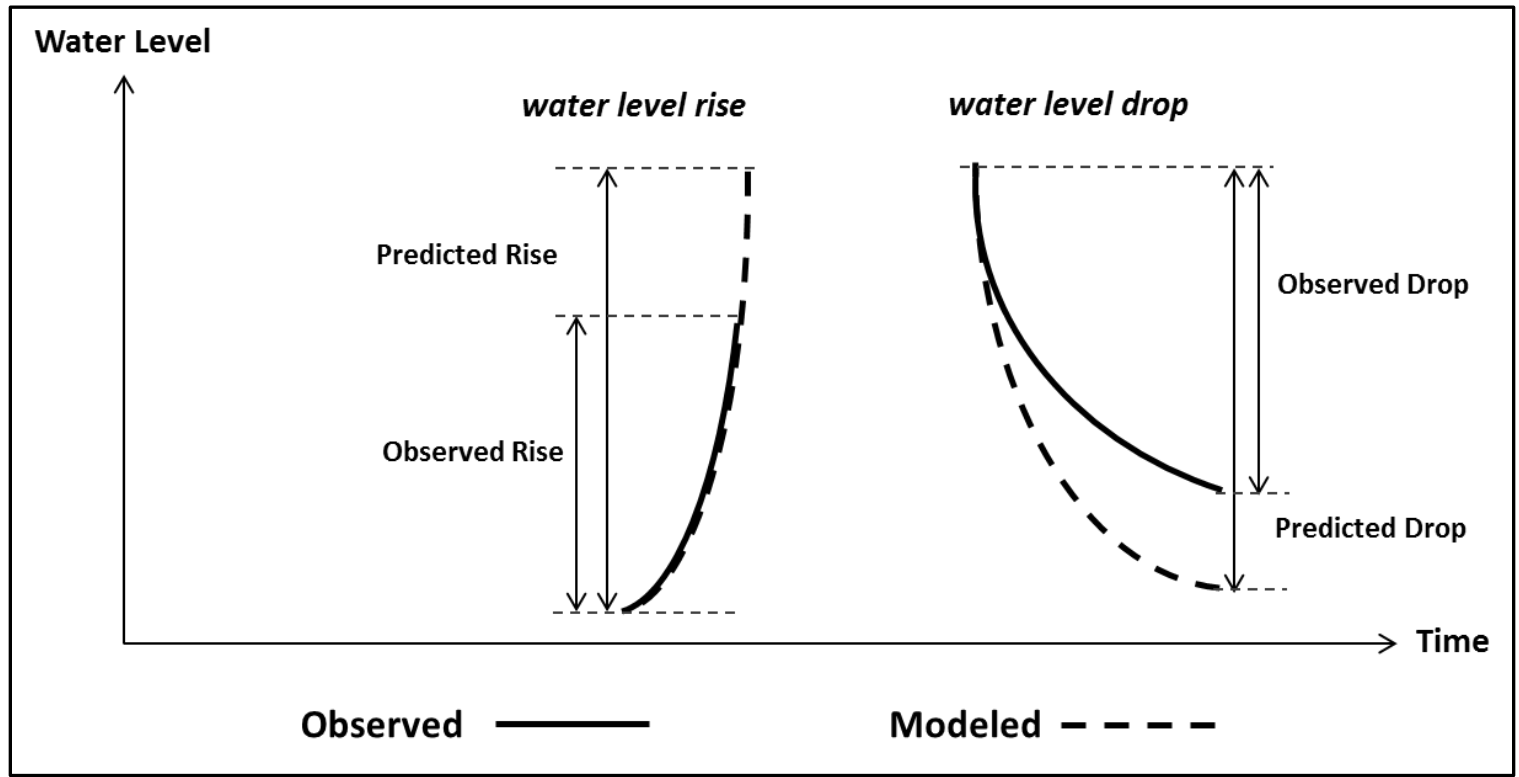

Figure 67 - The Model is Reset before Each Water Level Drop and Rise

Since the model has been calibrated with the initial hydrological performance of the GI controls, the modeling results indicate changes in infiltration capacity and exfiltration performance over time and compared to their baseline performance. All results from both modeling techniques are shown in Figures 68 through 75. It must be noted that all events with modeled drops and rises smaller than 50 centimeters are excluded from the results. This is done to make sure the margin of errors associated with limitations of models were minimal compared to the final calculated values. 


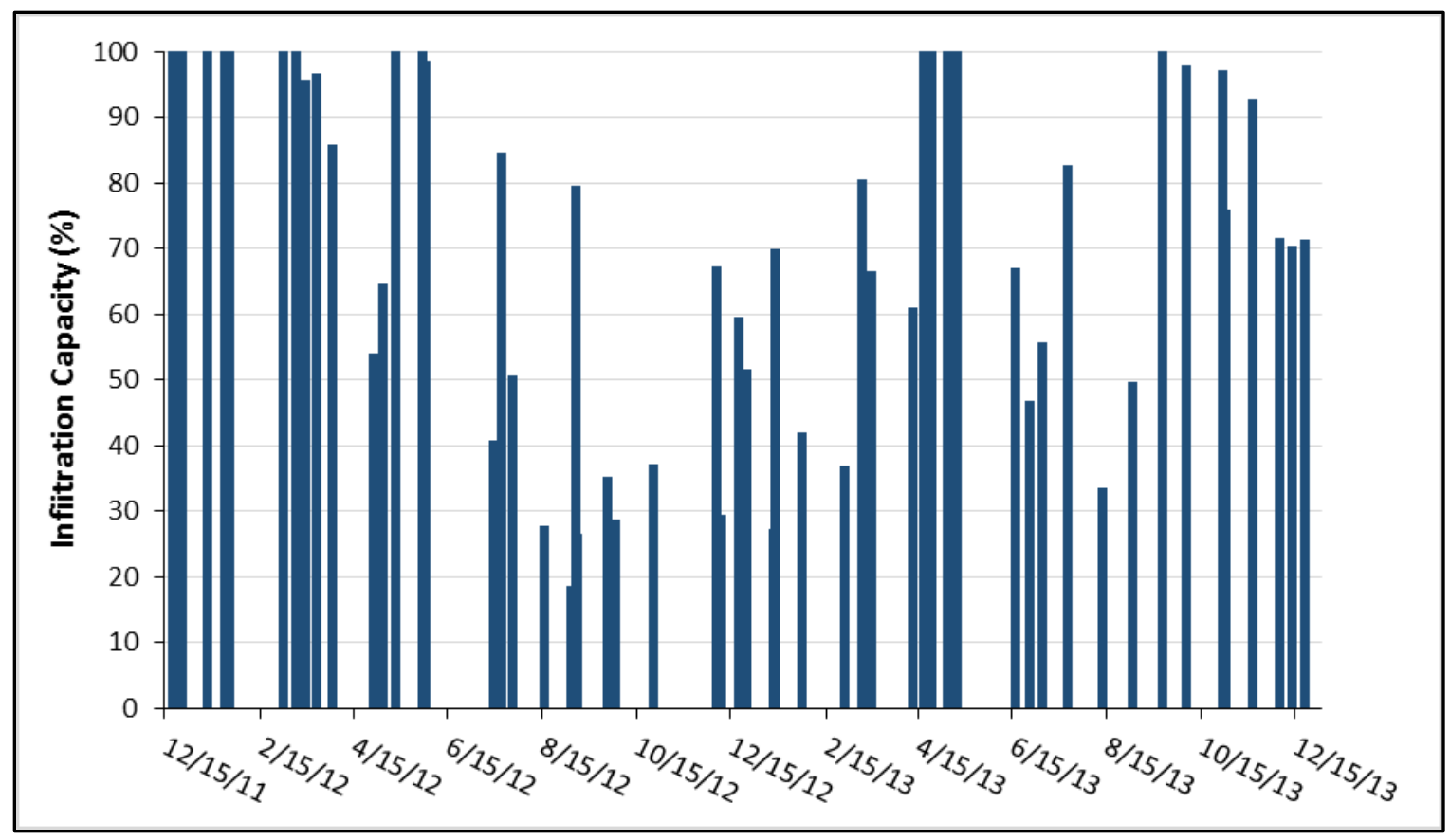

Figure 68 - Changes in Infiltration Capacity for Control 19G, Model by Technique 1

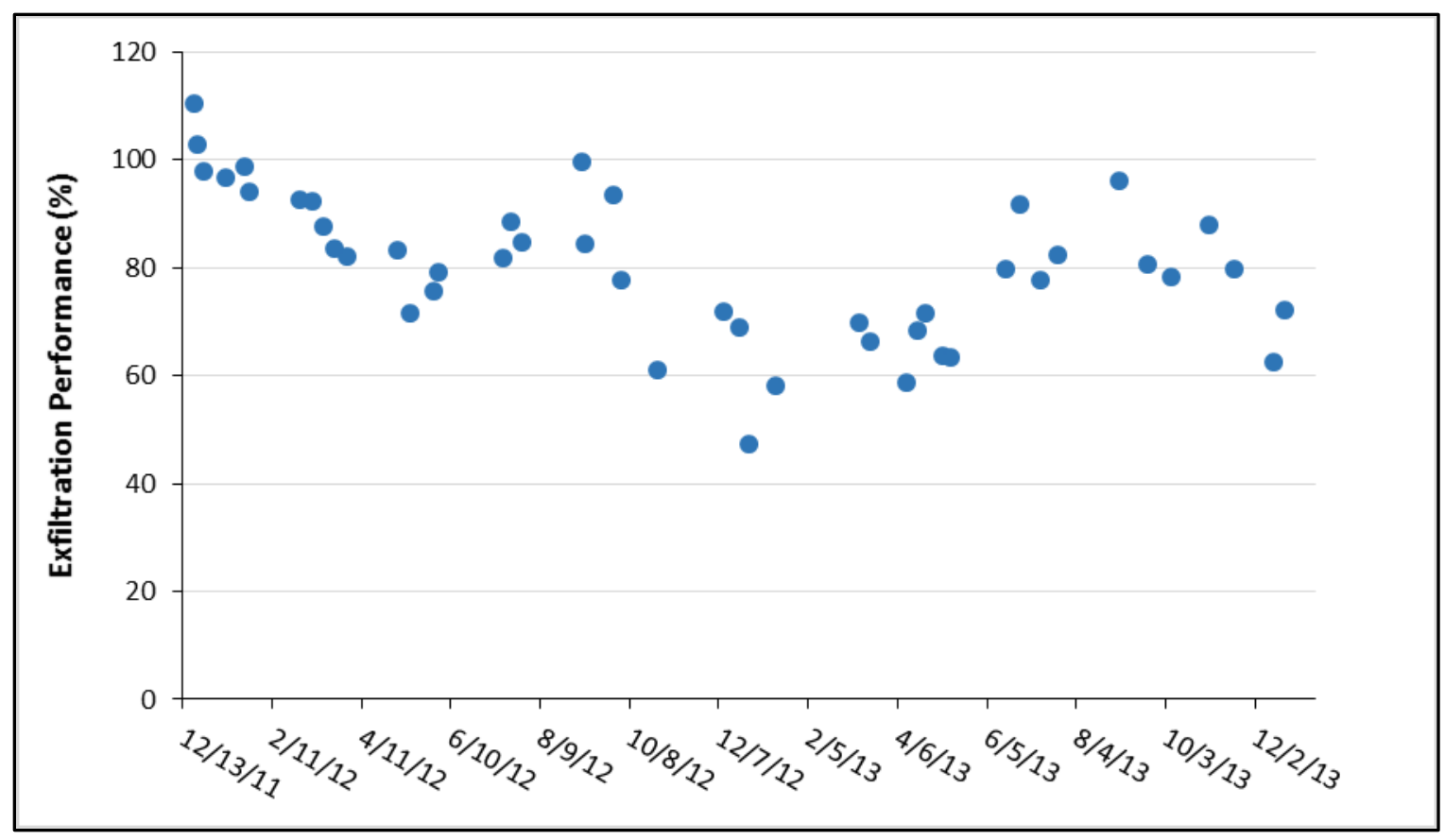

Figure 69 - Changes in Exfiltration Performance for Control 19G, Model by Technique 1 


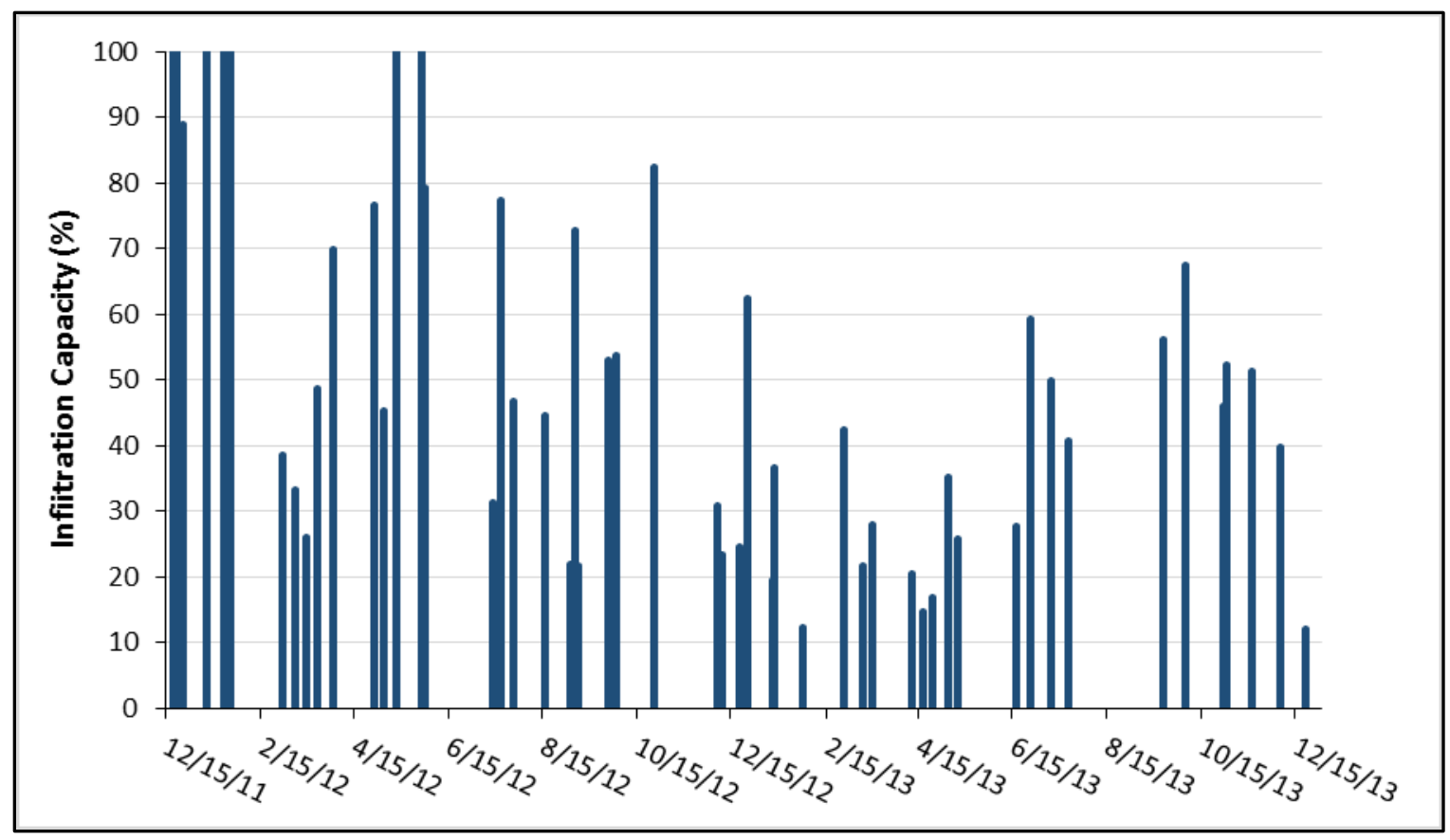

Figure 70 - Changes in Infiltration Capacity for Control 19H, Model by Technique 1

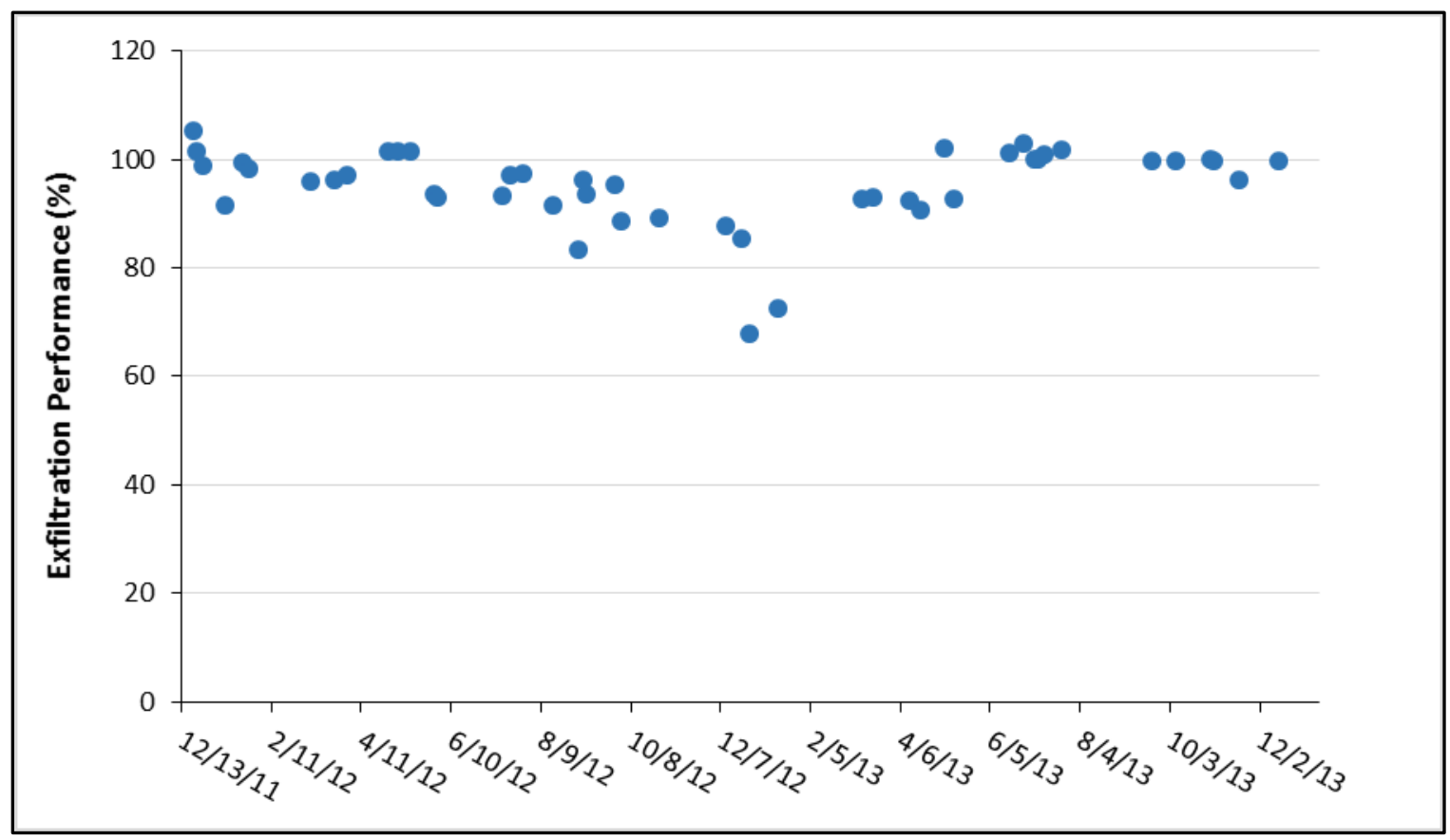

Figure 71 - Changes in Exfiltration Performance for Control 19H, Model by Technique 1 


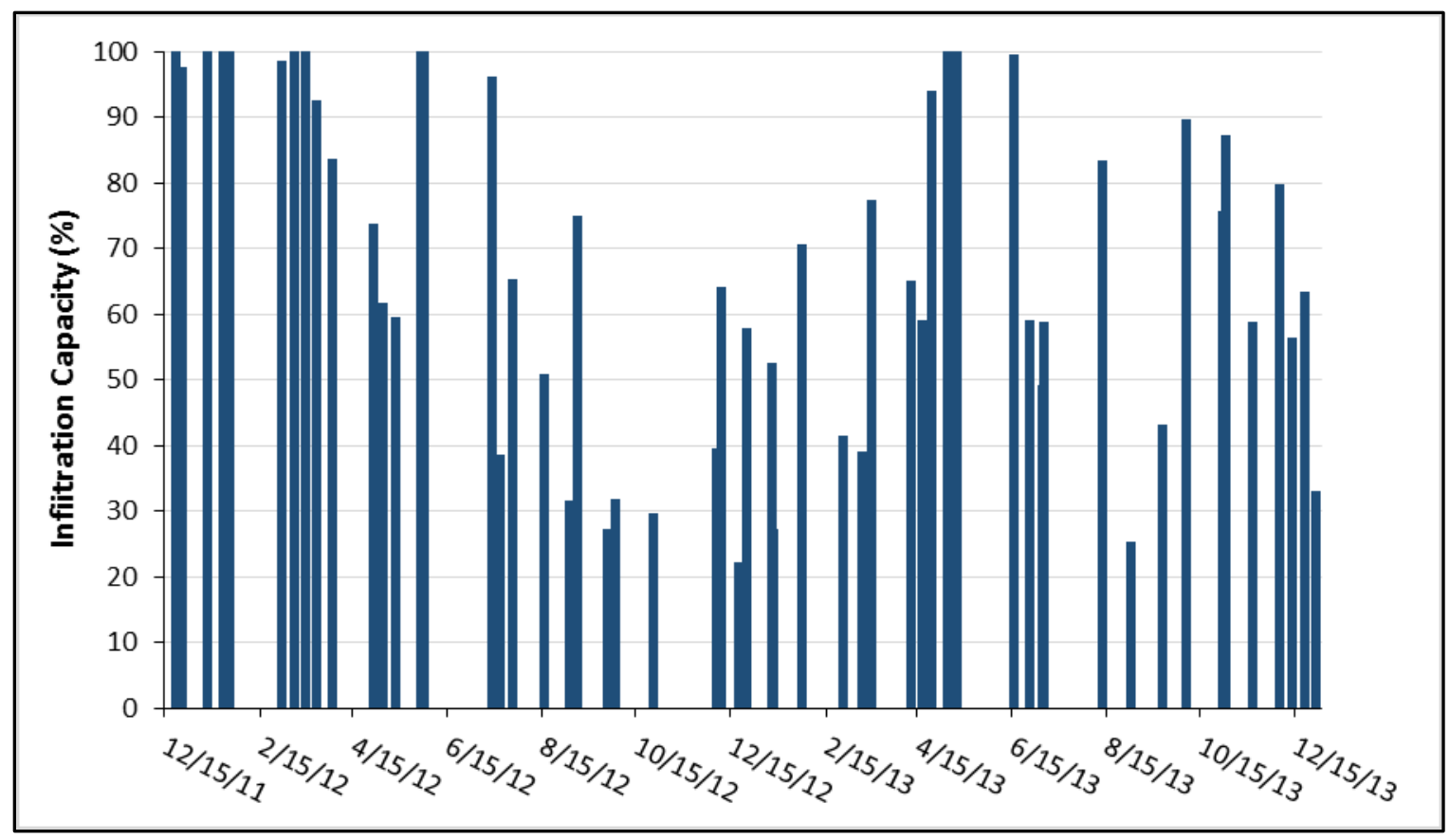

Figure 72 - Changes in Infiltration Capacity for Control 19G, Model by Technique 2

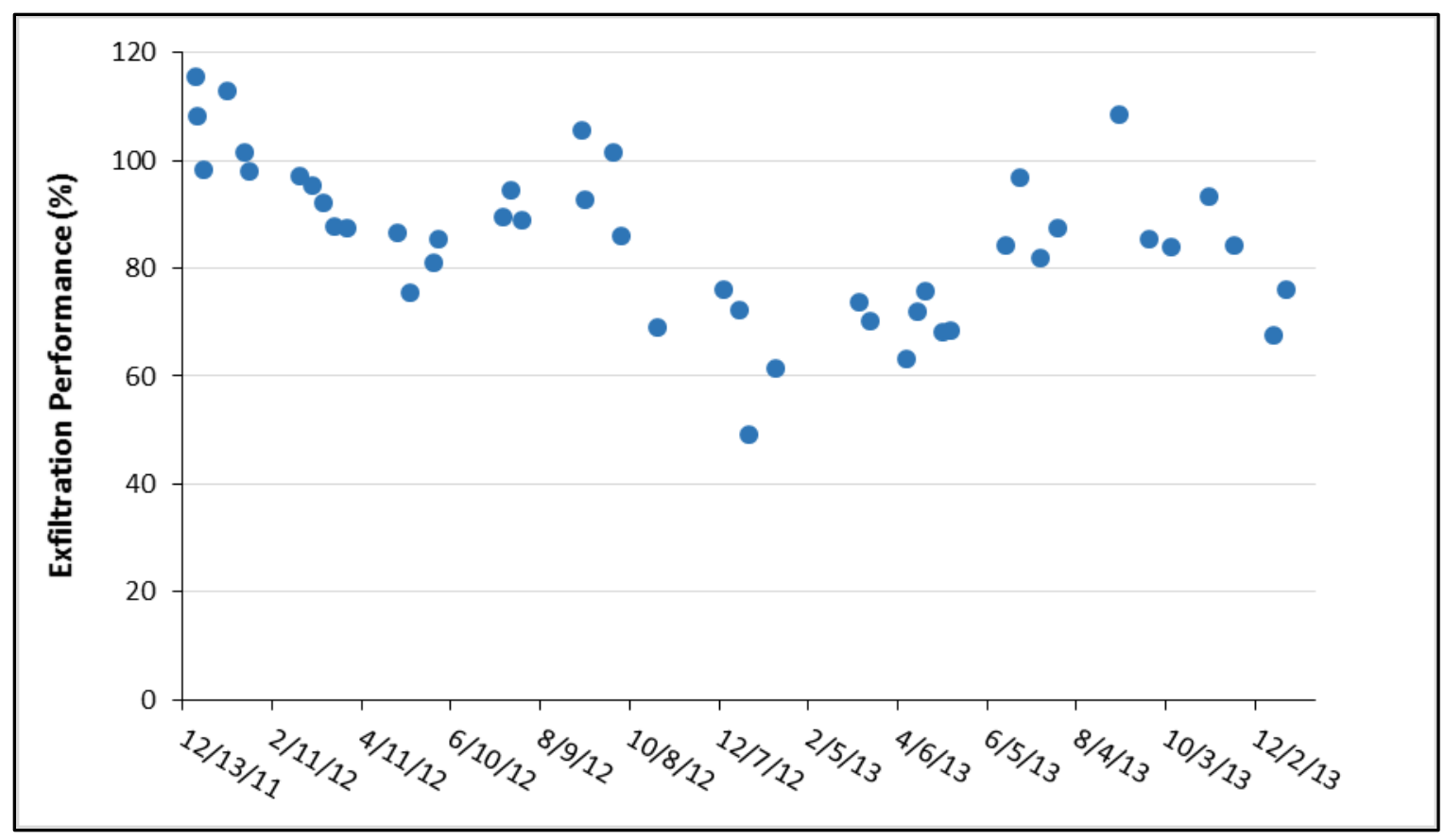

Figure 73 - Changes in Exfiltration Performance for Control 19G, Model by Technique 2 


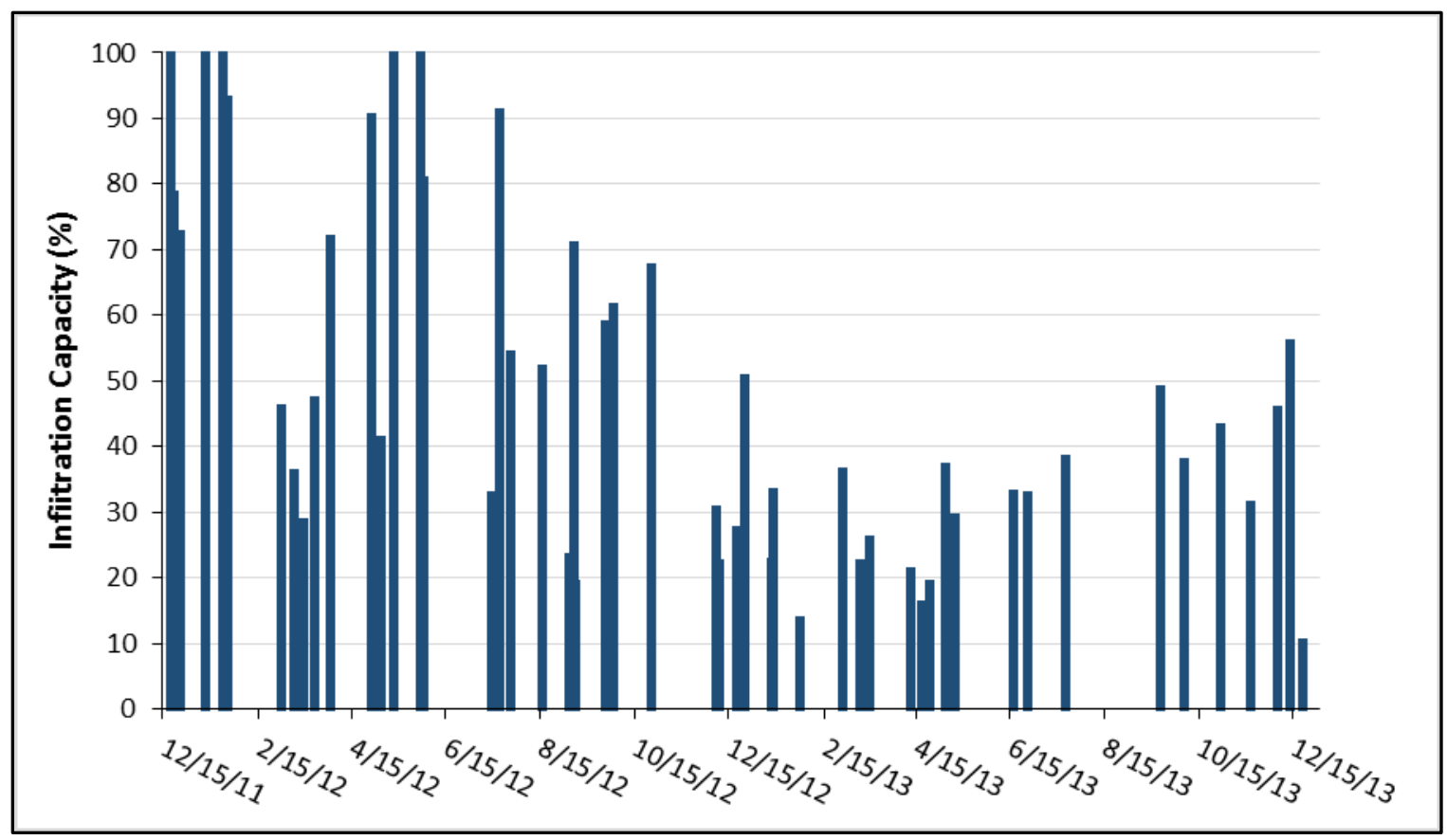

Figure 74 - Changes in Infiltration Capacity for Control 19H, Model by Technique 2

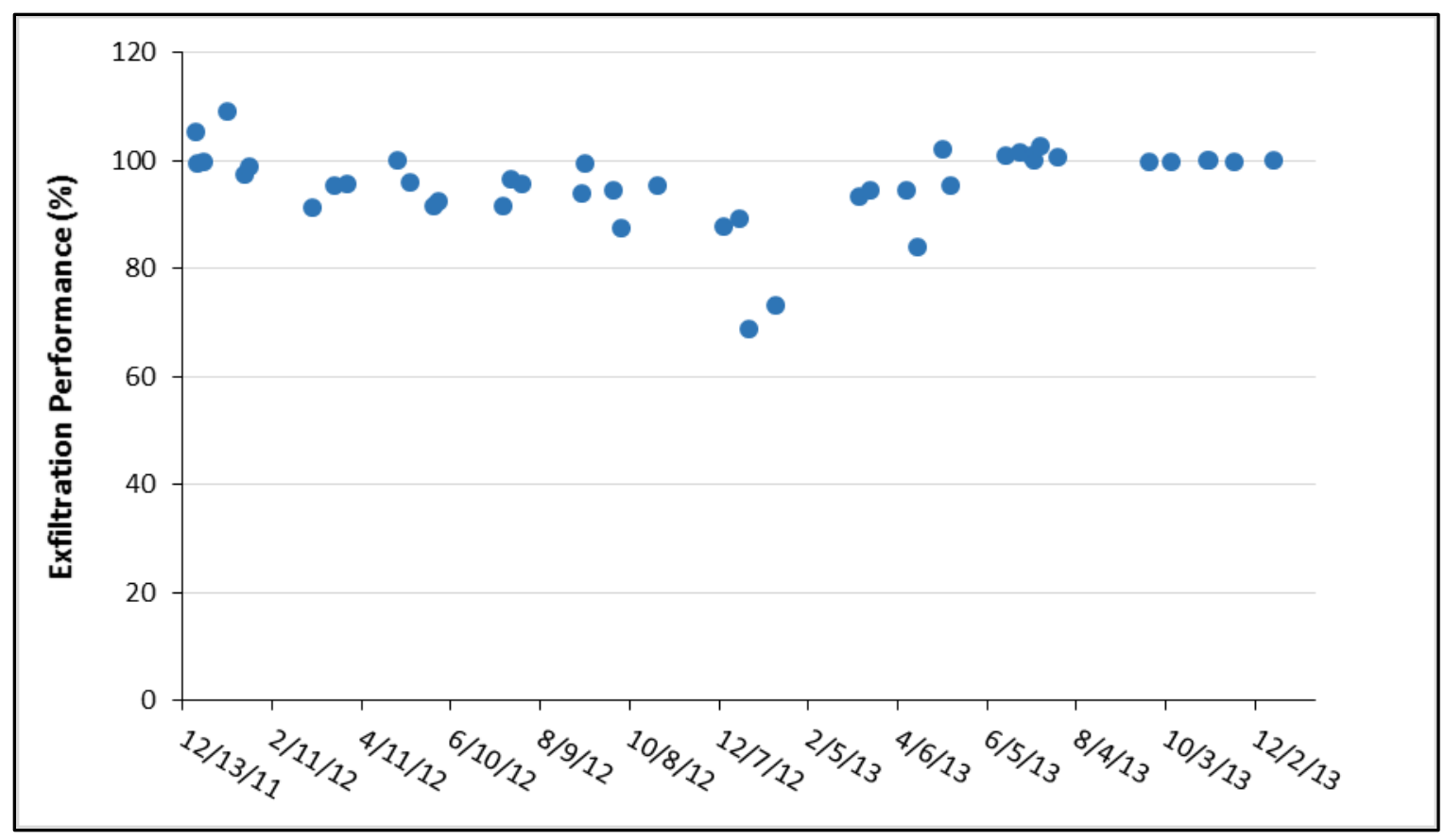

Figure 75 - Changes in Exfiltration Performance for Control 19H, Model by Technique 2 
As it can be seen from these figures, the results from both modeling techniques are in good agreement with each other. Infiltration capacity of both controls decreased over time following their construction and were only restored after conducting successful surface maintenance treatments. The figures showing changes in infiltration capacities are used to assess the long-term effectiveness of different maintenance methods and are discussed in detail in Chapter 6.

The changes in exfiltration performance from both GI controls are following the general trends observed in calculated exfiltration rates, see Figures 48 and 49. The calculated exfiltration rates show increases during summer time which results in restoration of exfiltration performance. Since the installation of GI controls (and subsequently the calibration of the model) was done in December and winter time, the observed restorations during summers of 2012 and 2013 doesn't indicate full restoration of exfiltration performances to their baseline level. While an initial significant decrease in exfiltration performances of both GI controls is observed in early 2012 no additional decrease is visible in late 2012 and 2013.

It should be pointed out that during the second half of 2013 a decrease in runoff volume into control 19G was observed which became quite apparent after the latest surface maintenance in September 2013. Following this maintenance treatment several field visits were done during the storm events to evaluate the restoration of infiltration capacity of Control 19G. These field observations and further analysis of surface TDRs showed that control 19G has been effectively able to capture all surface runoff from its drainage area and the clogging progression on the surface of this control has reverted to its initial pattern as previously explained in Section 4.3. On the other hand the modeling results from the 
original calibration showed conflicting results indicating that infiltration capacity is not completely restored. This was believed to be happening because of the construction of new GI controls in CSO130 basin in 2013. During this phase of construction a number of treeboxes were installed along the north side of Story Avenue which is located at upgradient of Adams Street with control 19G along its parking lane. An existing catch basin at the down gradient of these treeboxes were initially supposed to capture all surface runoff from north side of Story Avenue so this drainage area was excluded from contributing runoff to control 19G. It is suspected that this catch basin, similar to the one upgradient of control $19 \mathrm{H}$, has been bypassing some runoff volume toward control 19G and it was only after the construction of treeboxes that this runoff volume was completely captured before flowing into the Adams Street and toward control 19G.

To resolve this problem the drainage area in control 19G's model, developed from technique 1, was recalibrated for the first three rainfall events following the new maintenance treatment. The runoff volume is believed to be completely captured by control 19G during these three storm events. The recalibration showed that the drainage area for control 19G were decreased by approximately 36\% percent compared to 2011 and 2012 . Due to this recalibration there is an evident difference in infiltration performance of Control 19G in late 2013 from the two modeling techniques, see Figures 68 and 72.

\subsection{Captured Stormwater Volume}

The developed models were also utilized to estimate the volume captured by controls 19G and 19H while considering the intra-event exfiltration which were explained in Section 4.4.2. To do this, first, all increments of water level during rise were multiplied by dimensions of the GI controls and the porosity of the storage layers. Then the volume of 
water exfiltrated during the rise (intra-event exfiltration) was estimated by use of the developed models. The total volume captured was eventually estimated by adding the volume infiltrated (calculated from water level rise) and the volume exfiltrated during the rain event (intra-event exfiltration). Cumulative runoff and captured volumes for controls 19G and 19H are shown in Figures 76 and 77. As shown on these figures, both GI controls were able to capture all surface runoff initially and after their installation but as clogging on the surface of the permeable pavement systems reached the downgradient sides, the volume captured were decreased compared to the total runoff volume.

The total ratios of volumes captured to the total runoff volumes for controls $19 \mathrm{G}$ and 19H are shown in Figure 78. Control 19G has been able to capture more surface runoff from its drainage area compared to the control $19 \mathrm{H}$, which is partly because of the conducted surface maintenance treatments that are explained in more detail in Chapter 6. Figure 78 also shows the ratios of intra-event exfiltration volumes and the volumes calculated from water level rise to the total captured volume. As it can be seen almost $37 \%$ and $24 \%$ of the volumes captured were exfiltrated from controls $19 \mathrm{G}$ and $19 \mathrm{H}$ during the storm events which indicates that excluding the intra-event exfiltration from the calculations could have caused significant errors. 


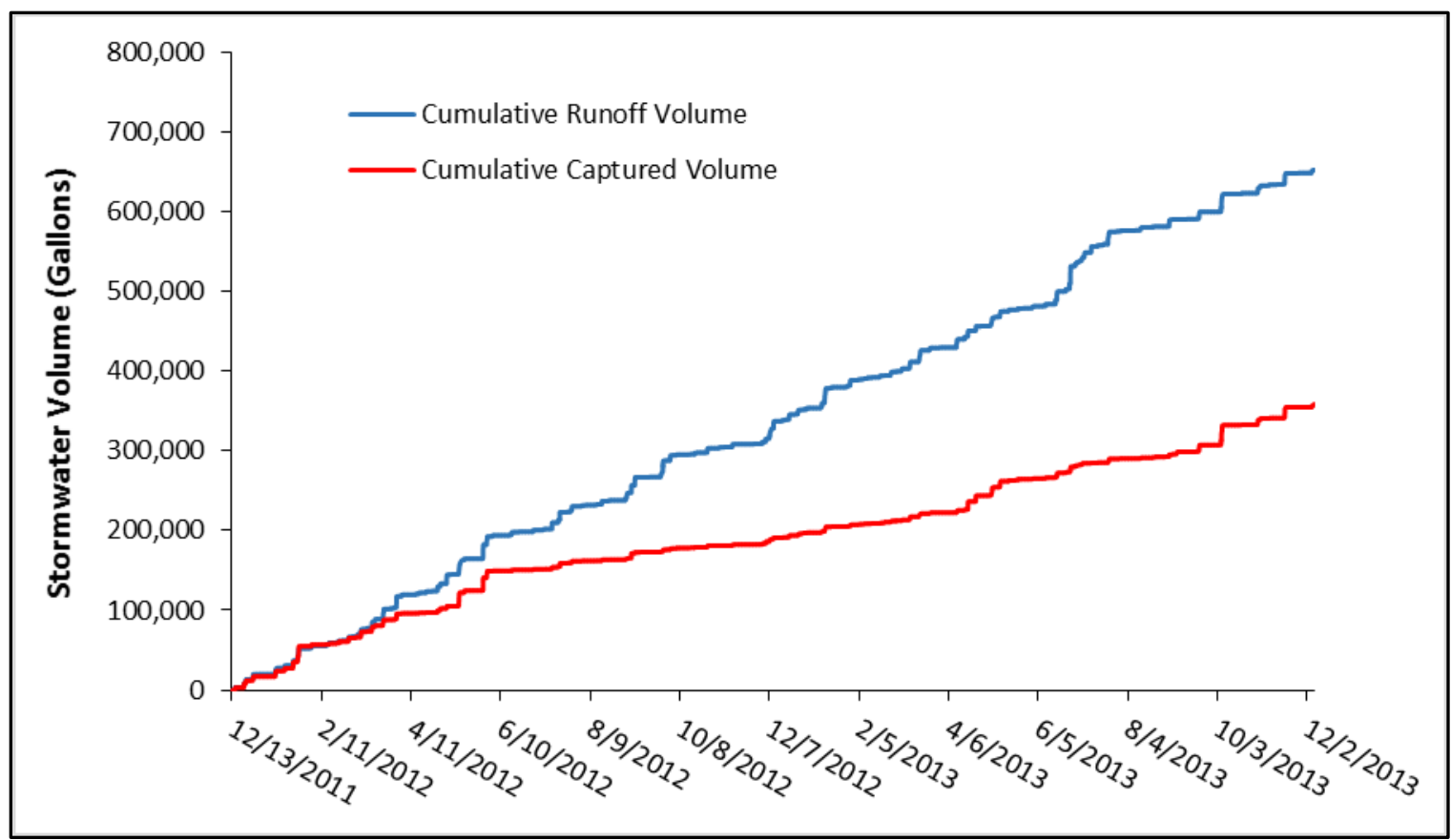

Figure 76 - Cumulative Runoff and Captured Volumes, Control 19G

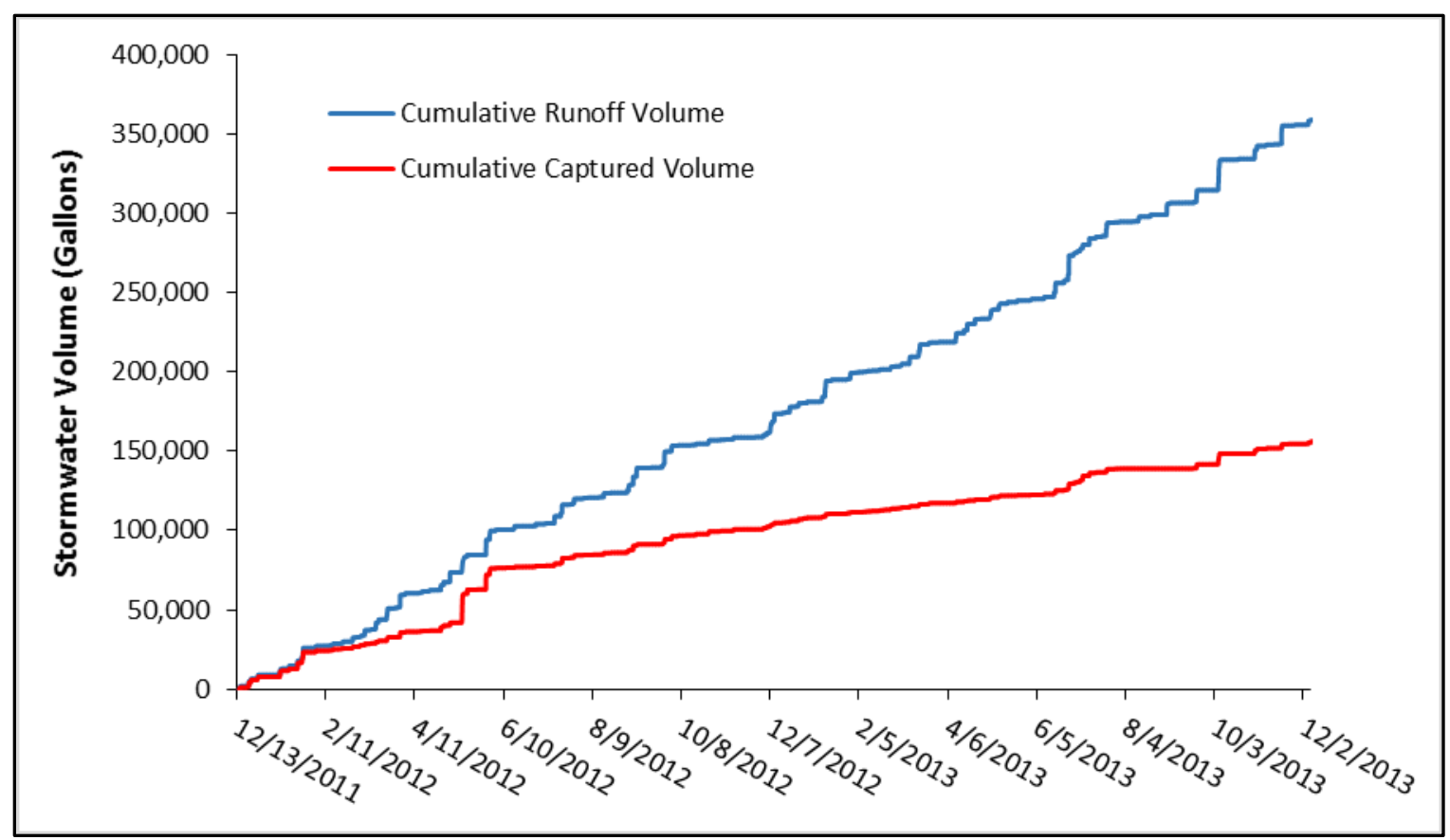

Figure 77 - Cumulative Runoff and Captured Volumes, Control 19G 


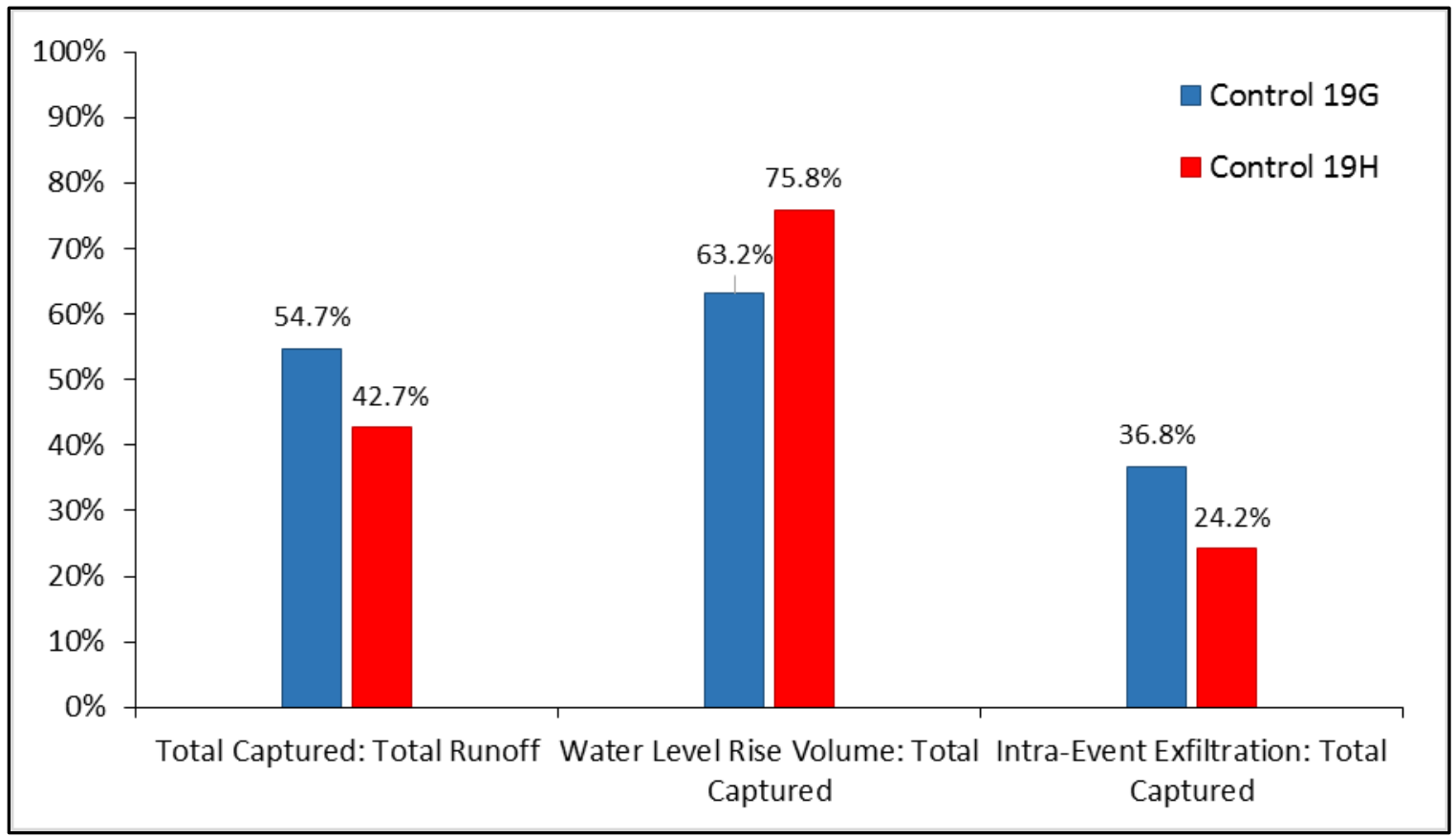

Figure 78 - Comparison between Infiltration Performances of Controls $19 \mathrm{G}$ and $19 \mathrm{H}$ and Role of Intra-event Exfiltration 


\section{CHANGES IN INFILTRATION CAPACITY AND SURFACE MAINTENANCE TREATMENTS}

This section explains the results from manual surface infiltration tests and the type and effectiveness of surface maintenance treatments that have been conducted on controls $19 \mathrm{G}$ and $19 \mathrm{H}$ during the course of this study. To assess the effectiveness of different maintenance methods, the results from surface infiltration tests, modeling effort explained in chapter 5 , and TDR data analysis are used.

\subsection{Manual Surface Infiltration Testing}

As mentioned in Chapter 3, following the construction of controls 19G and 19H, manual surface infiltration tests were completed to establish the baseline infiltration capacity of unclogged permeable pavements. The test locations on the surface of each GI control matched the location of TDRs installed between \#57 and \#3 stone layers, see Figure 15. Since over the course of study the runoff flow was observed to be concentrated along the curb edge of each GI control, the test locations for conducting these manual measurements were revised. The revised plan only included locations of curbside TDRs. The detailed method for conducting the surface infiltration tests is included in Appendix III. The results from initial surface infiltration tests are provided in Tables 21 and 22. In these tables each test location is identified with naming system shown in Figure 15, and X, Y coordinates measured from upgradient edge and curb side respectively. 
Table 21 - Initial Surface Infiltarion Test Results, Control 19G

\begin{tabular}{cccccc}
\hline Test Date & $\begin{array}{c}\text { Test } \\
\text { Location ID }\end{array}$ & $\mathbf{X}(\mathbf{f t})$ & $\mathbf{Y}(\mathbf{f t})$ & $\begin{array}{c}\text { Infiltration } \\
\text { Rate }(\mathbf{c m} / \mathbf{h r})\end{array}$ & $\begin{array}{c}\text { Infiltration } \\
\text { Rate }(\mathbf{i n} / \mathbf{h r})\end{array}$ \\
\hline $12 / 21 / 2011$ & 1-A & 2.5 & 0.5 & 948.1 & 383.1 \\
\hline $12 / 21 / 2011$ & 1-B & 2.5 & 4 & $3,354.7$ & $1,355.4$ \\
\hline $12 / 21 / 2011$ & $1-\mathrm{C}$ & 2.5 & 7.5 & $3,488.9$ & $1,409.7$ \\
\hline $12 / 21 / 2011$ & $2-\mathrm{A}$ & 7.5 & 0.5 & 807.6 & 326.3 \\
\hline $12 / 21 / 2011$ & 2-B & 7.5 & 4 & $2,422.8$ & 978.9 \\
\hline $12 / 21 / 2011$ & 2-C & 7.5 & 7.5 & $2,565.4$ & $1,036.5$ \\
\hline $12 / 21 / 2011$ & $3-\mathrm{A}$ & 40 & 0.5 & $2,813.6$ & $1,136.8$ \\
\hline $12 / 21 / 2011$ & $3-\mathrm{B}$ & 40 & 4 & $2,725.7$ & $1,101.3$ \\
\hline $12 / 21 / 2011$ & 3-C & 40 & 7.5 & $2,813.6$ & $1,136.8$ \\
\hline $1 / 6 / 2012$ & $4-\mathrm{A}$ & 75 & 0.5 & $3,007.7$ & $1,215.2$ \\
\hline $1 / 6 / 2012$ & $4-\mathrm{B}$ & 75 & 4 & $2,565.4$ & $1,036.5$ \\
\hline $1 / 6 / 2012$ & $4-\mathrm{C}$ & 75 & 7.5 & $3,488.9$ & $1,409.7$ \\
\hline
\end{tabular}

Table 22 - Initial Surface Infiltarion Test Results, Control 19H

\begin{tabular}{cccccc}
\hline Test Date & $\begin{array}{c}\text { Test } \\
\text { Location ID }\end{array}$ & $\mathbf{X}(\mathbf{f t})$ & $\mathbf{Y}(\mathbf{f t})$ & $\begin{array}{c}\text { Infiltration } \\
\text { Rate }(\mathbf{c m} / \mathbf{h r})\end{array}$ & $\begin{array}{c}\text { Infiltration } \\
\text { Rate }(\mathbf{i n} / \mathbf{h r})\end{array}$ \\
\hline $12 / 23 / 2011$ & 1-A & 2.5 & 0.5 & 379.2 & 153.2 \\
\hline $12 / 23 / 2011$ & $1-\mathrm{B}$ & 2.5 & 4 & $3,488.9$ & $1,409.7$ \\
\hline $12 / 23 / 2011$ & $1-\mathrm{C}$ & 2.5 & 7.5 & $3,634.2$ & $1,468.4$ \\
\hline $12 / 23 / 2011$ & $2-\mathrm{A}$ & 7.5 & 0.5 & 55.6 & 22.4 \\
\hline $12 / 23 / 2011$ & 2-B & 7.5 & 4 & $3,488.9$ & $1,409.7$ \\
\hline $12 / 23 / 2011$ & 2-C & 7.5 & 7.5 & $3,488.9$ & $1,409.7$ \\
\hline $12 / 23 / 2011$ & $3-\mathrm{A}$ & 20 & 0.5 & $3,354.7$ & $1,355.4$ \\
\hline $12 / 23 / 2011$ & $3-\mathrm{B}$ & 20 & 4 & $3,488.9$ & $1,409.7$ \\
\hline $12 / 23 / 2011$ & 3-C & 20 & 7.5 & $3,634.2$ & $1,468.4$ \\
\hline $12 / 23 / 2011$ & $4-\mathrm{A}$ & 40 & 0.5 & $3,354.7$ & $1,355.4$ \\
\hline $12 / 23 / 2011$ & $4-\mathrm{B}$ & 40 & 4 & $3,488.9$ & $1,409.7$ \\
\hline $12 / 23 / 2011$ & $4-\mathrm{C}$ & 40 & 7.5 & $3,634.2$ & $1,468.4$ \\
\hline
\end{tabular}

As it can be seen in these tables, the surface infiltration measurements were conducted almost 10 days after the installation of the GI controls. In this period controls 19G and 19H had experienced two (total depth 1.12 inches) and three rainfall events (total depth 1.73 inches), respectively. Visual inspections and TDR data analysis indicated that surface clogging had started to advance on the surface of both control before conducting 
the surface measurements. Therefore the most upgradient data points with lowest infiltration rates from each table (shaded in gray) were suspected to be invalid, not representing the baseline infiltration rates of permeable pavements. To statistically confirm this, unpaired student's t-tests were performed and these point were confirmed to have significantly smaller infiltration rates compared to others, with P-values equal to 0.00 and 0.03 for Tables 21 and 22, respectively. By excluding these data points the average baseline infiltration rates for each GI control were decided which are shown in Table 23.

Table 23 - Average of Baseline Infiltration Rates for Controls 19G and 19H

\begin{tabular}{lcccc}
\hline & \multicolumn{2}{c}{ Control 19G } & \multicolumn{2}{c}{ Control 19H } \\
\cline { 2 - 5 } & $\begin{array}{c}\text { Infiltration } \\
\text { Rate }(\mathrm{cm} / \mathrm{hr})\end{array}$ & $\begin{array}{c}\text { Infiltration } \\
\text { Rates }(\mathrm{in} / \mathrm{hr})\end{array}$ & $\begin{array}{c}\text { Infiltration } \\
\text { Rate }(\mathrm{cm} / \mathrm{hr})\end{array}$ & $\begin{array}{c}\text { Infiltration } \\
\text { Rates (in/hr) }\end{array}$ \\
\hline Mean & 2980.4 & 1204.7 & 3505.7 & 1416.5 \\
\hline Median & 2813.6 & 1136.8 & 3488.9 & 1409.7 \\
\hline Standard Deviation & 374.7 & 159.5 & 103.6 & 41.9 \\
\hline Minimum & 2565.4 & 1036.5 & 3354.7 & 1355.4 \\
\hline Maximum & 3488.9 & 1409.7 & 3634.2 & 1468.4 \\
\hline
\end{tabular}

Following the construction of the controls 19G and 19H in December 2011, visual field inspections and the electronic data showed that clogging advanced on the surface of both GI controls from the upgradient edge toward the downgradient edge, see Figure 79. 


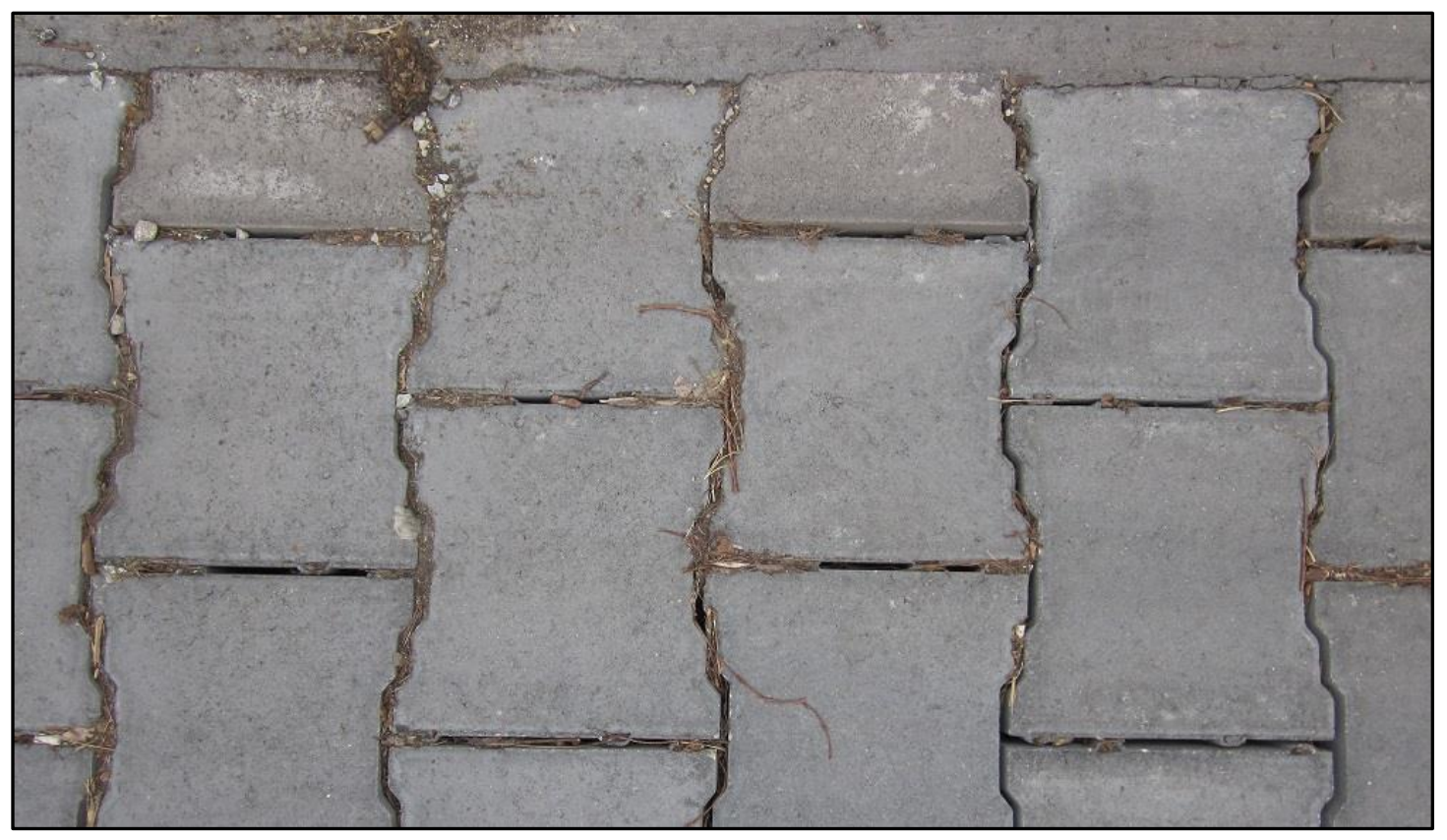

Figure 79 - Observed Surface Clogging on the Surface of Control 19G, February 2012

\subsection{Maintenance Treatment Methods and their Effectiveness}

To restore the infiltration capacity of controls $19 \mathrm{G}$ and $19 \mathrm{H}$, several maintenance treatment methods were conducted during the course of this study. In this section these methods are explained and studied to determine which one was most effective. The effectiveness of a method can be determined for short-term and long-term conditions but a method with more long-term effectiveness is clearly preferred. After installation of GI controls in 2011 through the end of 2013, a total of five and three maintenance treatments were conducted on controls $19 \mathrm{G}$ and $19 \mathrm{H}$. These maintenance efforts consisted of three different methods included using: a conventional street sweeper/vacuum truck, a pressurized air jet, and a prototype attachments for hydro excavation trucks (Vac-Head method). These methods are explained briefly as follows: 
- Street Sweeper/Vacuum Truck: this was the first maintenance method that were tested and it was conducted on control 19G only and by using a street sweeper truck (Isuzu model NQR 435). In this method the truck applies a combination of vacuuming and mechanical sweeping to clean the gaps of permeable pavement. The sweeper truck cleaned the surface of the pavement four times over the entire length of the control 19G.

- Pressurized Airjet: this method which was conducted on both GI controls, includes using a high pressurized airjet to manually blow out the sediments trapped between the gaps of the permeable pavement. This method were repeated three times over the course of this study. After the first maintenance with this method, a street sweeper made one pass over the surface of the GI controls to collect the discharged sediments from the permeable pavements' gaps.

- Vac-Head Method: this was the last maintenance treatment used to restore the surface infiltration rates of the permeable pavements and was only conducted on control 19G. This method, which was devised and recommended by the manufacturer of the permeable pavements (PaveDrain ${ }^{\circledR}$ LLC), included using a prototype attachment that were connected to a hydro excavation truck, common in the sewer service industry. The attachment, which is controlled manually, used two rotating water jets to dislodge the trapped sediments out of the gaps of permeable pavements and vacuumed concurrently to capture the mix of water and dislodged clogging material. Since this method utilizes a combination of 
water head and vacuum to clean the pavements, per suggestion of the manufacturer of the pavements, it is referred to as "Vac-Head" method.

Table 24 provides the number of maintenance methods and their employment dates. The rainfall data between each maintenance treatment is included as well. Figure 80 shows photos from these three different maintenance methods. Figure 81 shows photos from pre and post maintenance treatments. To evaluate the effectiveness of these maintenance treatments, three different assessment techniques are used. These techniques include using the surface infiltration test results, TDR data analysis, and using the infiltration capacity results from modeling effort explained in Chapter 5.

Table 24 - Summary of Maintenance Treatments

\begin{tabular}{lcccc}
\hline \multicolumn{1}{c}{ Maintenance Type } & Date & $\begin{array}{c}\text { Control } \\
\text { ID }\end{array}$ & $\begin{array}{c}\text { Days Since } \\
\text { Installation/Last } \\
\text { Maintenance }\end{array}$ & $\begin{array}{c}\text { Rainfall Depth Since } \\
\text { Installation/Last } \\
\text { Maintenance (Inches) }\end{array}$ \\
\hline Sweeper/Vacuum Truck & $03 / 20 / 2012$ & $19 \mathrm{G}$ & 97 & 11.1 \\
\hline Pressurized Airjet \#1 & $05 / 09 / 2012$ & $19 \mathrm{G}, 19 \mathrm{H}$ & 51 & 6.8 \\
\hline Pressurized Airjet \#2 & $10 / 05 / 2012$ & $19 \mathrm{G}, 19 \mathrm{H}$ & 149 & 17.8 \\
\hline Pressurized Airjet \#3 & $04 / 17 / 2013$ & $19 \mathrm{G}, 19 \mathrm{H}$ & 194 & 19.63 \\
\hline Vac-Head & $09 / 18 / 2013$ & $19 \mathrm{G}$ & 154 & 19.53 \\
\hline
\end{tabular}




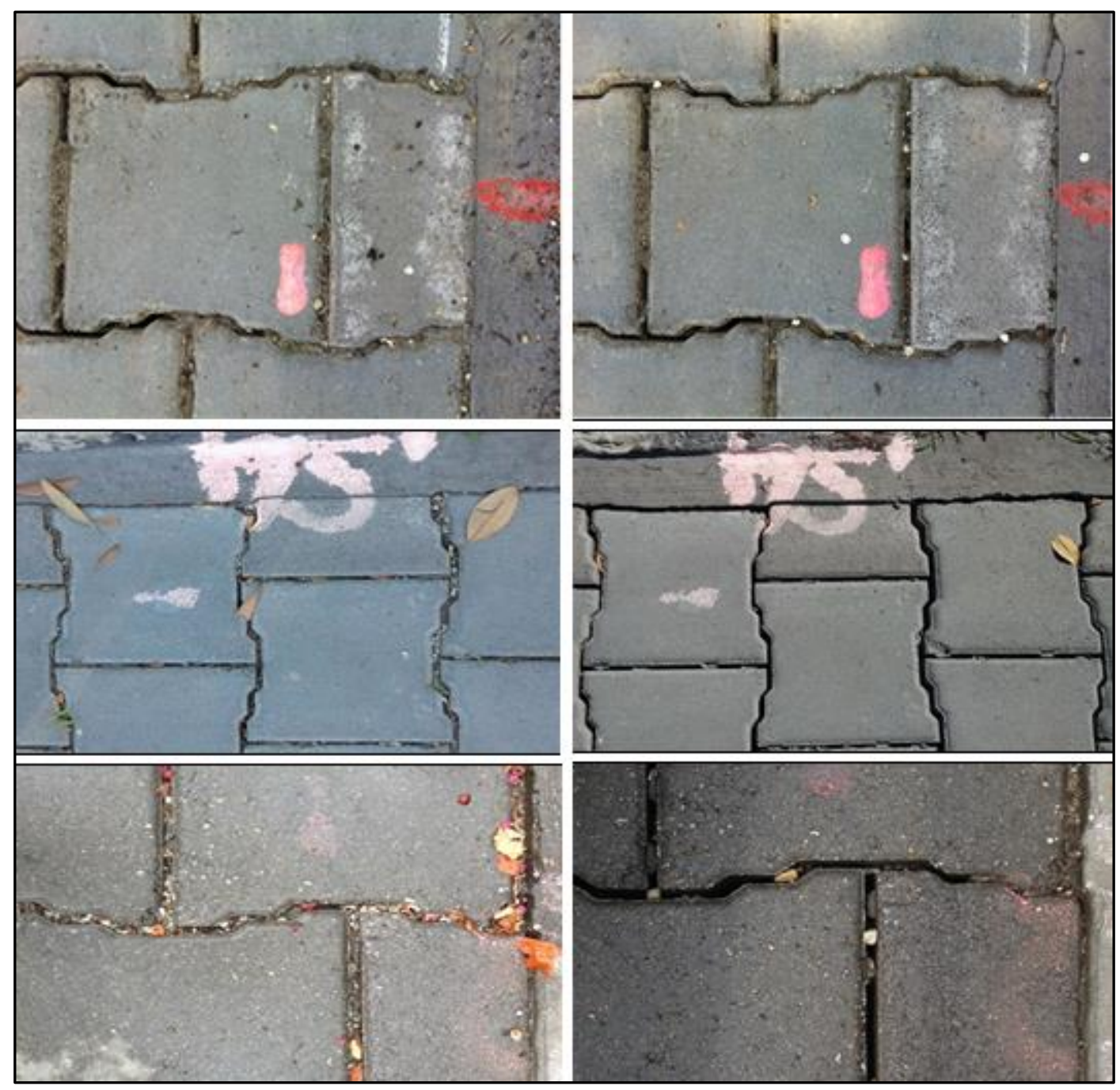

Figure 80 - Pre (left) and Post (right) Maintenance Photos. Sweeper/Vacuum Truck (top). Pressurized Airjet \#1 (middle), and Vac-Head (bottom) 


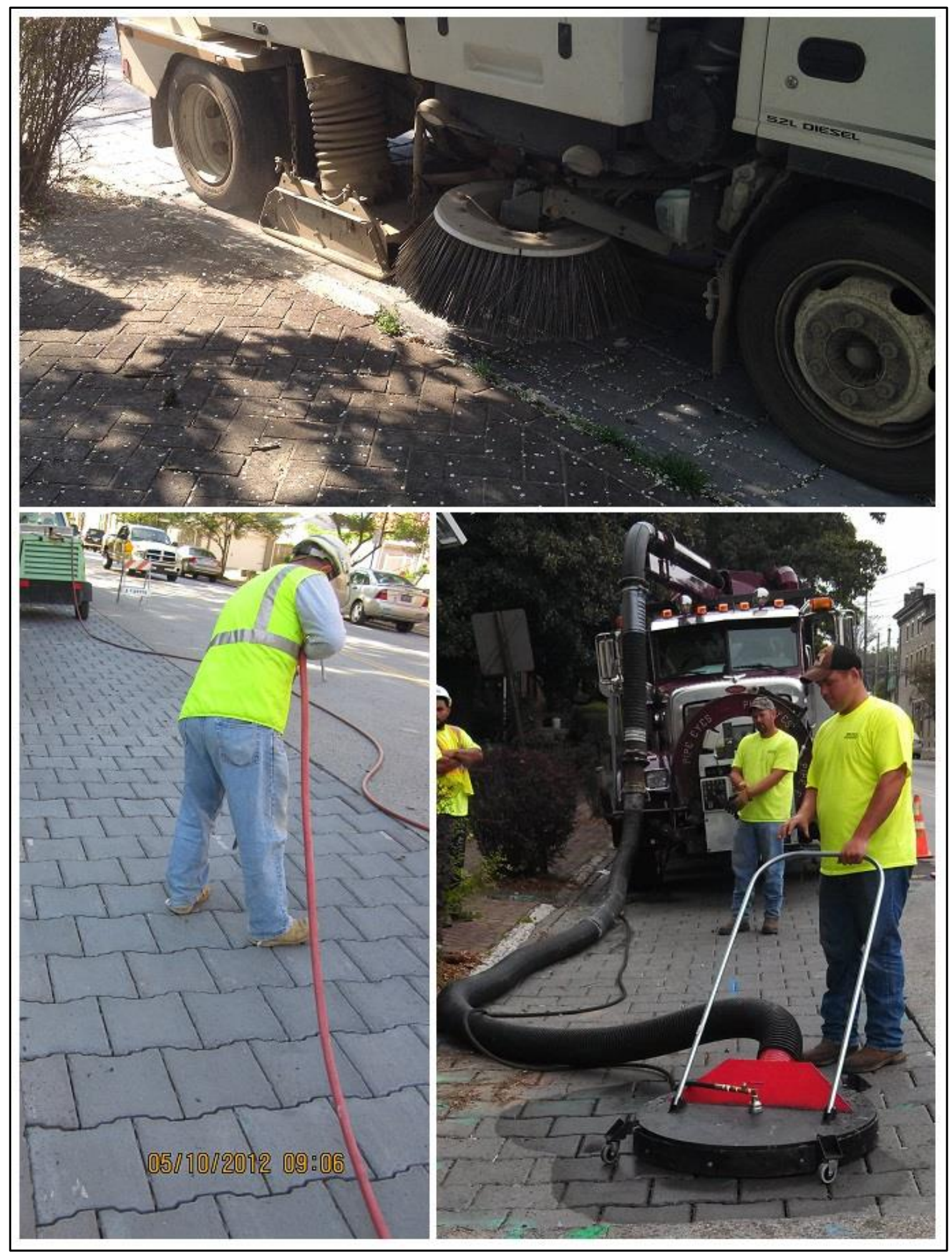

Figure 81 - The Three Methods Used for Restoring Infiltration Capacity of Controls $19 \mathrm{G}$ and $19 \mathrm{H}$ :

Sweeper/Vacuum Truck (up), Pressurized Airjet (bottom, left), and Vac-Head (bottom, right) 


\subsubsection{Surface Infiltration Test Results}

As previously mentioned, manual surface infiltration tests were conducted on controls 19G and 19H within one week before and after each maintenance treatment. The pre and post maintenance measured surface infiltration rates are included in Tables 25 to 28. Since UofL wasn't notified prior to Pressurized Airjet Maintenance in April 2013 no testing were conducted before and after this treatment. It must be noted that the results from surface infiltration tests only assess the restoration of infiltration capacity over the test locations and not the total surface area of permeable pavement. Also the results from these tests only represent short-term effectiveness of each maintenance treatment and use of results from TDR analysis and modeling effort is recommended to evaluate the long-term effectiveness of these methods. As it can be seen in Tables 25 to 28 , the restoration in infiltration rates can be observed with all maintenance treatments.

Table 25 - Pre and Post Maintenance Surface Infiltration Test Results, Sweeper Vacuum Truck

\begin{tabular}{|c|c|c|c|c|c|c|}
\hline \multicolumn{7}{|c|}{ Control 19G } \\
\hline $\begin{array}{c}\text { Test } \\
\text { Location }\end{array}$ & $\mathbf{X}(\mathbf{f t})$ & $\mathbf{Y}(\mathbf{f t})$ & $\begin{array}{c}\text { Pre- } \\
\text { Maintenance } \\
\text { Date }\end{array}$ & $\begin{array}{c}\text { Measured } \\
\text { Infiltration Rate } \\
(\text { in/hr })\end{array}$ & $\begin{array}{c}\text { Post } \\
\text { Maintenance } \\
\text { Date }\end{array}$ & $\begin{array}{c}\text { Measured } \\
\text { Infiltration } \\
\text { Rate } \\
\text { (in/hr) }\end{array}$ \\
\hline $1-\mathrm{A}$ & 2.5 & 0.5 & $3 / 7 / 2012$ & 6.0 & $3 / 21 / 2012$ & 340.5 \\
\hline $1-B$ & 2.5 & 4 & $3 / 7 / 2012$ & 429.8 & $3 / 21 / 2012$ & 496.4 \\
\hline $1-\mathrm{C}$ & 2.5 & 7.5 & $3 / 7 / 2012$ & $1,006.9$ & $3 / 21 / 2012$ & $1,456.3$ \\
\hline $2-\mathrm{A}$ & 7.5 & 0.5 & $3 / 7 / 2012$ & 19.5 & $3 / 21 / 2012$ & 124.7 \\
\hline $2-\mathrm{C}$ & 7.5 & 7.5 & $3 / 7 / 2012$ & 978.9 & $3 / 21 / 2012$ & $1,552.5$ \\
\hline 4-A & 40 & 0.5 & $3 / 7 / 2012$ & 130.3 & $3 / 21 / 2012$ & 906.0 \\
\hline $4-C$ & 40 & 7.5 & $3 / 7 / 2012$ & $1,350.3$ & $3 / 21 / 2012$ & $1,393.0$ \\
\hline
\end{tabular}


Table 26 - Pre and Post Maintenance Surface Infiltration Test Results, Pressurized Airjet \#1

\begin{tabular}{|c|c|c|c|c|c|c|}
\hline \multicolumn{7}{|c|}{ Control 19G } \\
\hline $\begin{array}{c}\text { Test } \\
\text { Location }\end{array}$ & $X$ (ft) & $\mathbf{Y}(\mathbf{f t})$ & $\begin{array}{c}\text { Pre- } \\
\text { Maintenance } \\
\text { Date }\end{array}$ & $\begin{array}{c}\text { Measured } \\
\text { Infiltration Rate } \\
(\text { in/hr })\end{array}$ & $\begin{array}{c}\text { Post } \\
\text { Maintenance } \\
\text { Date }\end{array}$ & $\begin{array}{c}\text { Measured } \\
\text { Infiltration } \\
\text { Rate } \\
\text { (in/hr) }\end{array}$ \\
\hline $1-\mathrm{A}$ & 2.5 & 0.5 & $5 / 7 / 2012$ & 18.3 & $5 / 11 / 2012$ & 203.7 \\
\hline $1-B$ & 2.5 & 4 & $5 / 7 / 2012$ & 677.7 & $5 / 11 / 2012$ & $1,006.9$ \\
\hline $1-\mathrm{C}$ & 2.5 & 7.5 & $5 / 7 / 2012$ & $1,957.9$ & $5 / 11 / 2012$ & $2,188.9$ \\
\hline $2-\mathrm{A}$ & 7.5 & 0.5 & $5 / 7 / 2012$ & 89.2 & $5 / 11 / 2012$ & $1,329.9$ \\
\hline $2-\mathrm{C}$ & 7.5 & 7.5 & $5 / 7 / 2012$ & 87.0 & $5 / 11 / 2012$ & $1,854.8$ \\
\hline 4-A & 40 & 0.5 & $5 / 7 / 2012$ & 261.0 & $5 / 11 / 2012$ & $1,654.5$ \\
\hline $4-\mathrm{C}$ & 40 & 7.5 & $5 / 7 / 2012$ & $1,468.4$ & $5 / 11 / 2012$ & $1,468.4$ \\
\hline \multicolumn{7}{|c|}{ Control 19H } \\
\hline $1-\mathrm{A}$ & 2.5 & 0.5 & $5 / 7 / 2012$ & 235.0 & $5 / 11 / 2012$ & $1,968.8$ \\
\hline $1-\mathrm{B}$ & 2.5 & 4 & $5 / 7 / 2012$ & 36.0 & $5 / 11 / 2012$ & $2,073.0$ \\
\hline $1-\mathrm{C}$ & 2.5 & 7.5 & $5 / 7 / 2012$ & 496.4 & $5 / 11 / 2012$ & $2,060.9$ \\
\hline $2-\mathrm{A}$ & 7.5 & 0.5 & $5 / 7 / 2012$ & 167.8 & $5 / 11 / 2012$ & $2,013.8$ \\
\hline $2-\mathrm{C}$ & 7.5 & 7.5 & $5 / 7 / 2012$ & 176.2 & $5 / 11 / 2012$ & $1,438.4$ \\
\hline 4-A & 40 & 0.5 & $5 / 7 / 2012$ & 30.5 & $5 / 11 / 2012$ & $1,136.8$ \\
\hline $4-\mathrm{C}$ & 40 & 7.5 & $5 / 7 / 2012$ & 542.2 & $5 / 11 / 2012$ & $1,566.3$ \\
\hline
\end{tabular}

Table 27 - Pre and Post Maintenance Surface Infiltration Test Results, Pressurized Airjet \#2

\begin{tabular}{|c|c|c|c|c|c|c|}
\hline \multicolumn{7}{|c|}{ Control 19G } \\
\hline $\begin{array}{c}\text { Test } \\
\text { Location }\end{array}$ & $\mathbf{X}(\mathbf{f t})$ & $\mathbf{Y}(\mathbf{f t})$ & $\begin{array}{c}\text { Pre- } \\
\text { Maintenance } \\
\text { Date }\end{array}$ & $\begin{array}{c}\text { Measured } \\
\text { Infiltration Rate } \\
\quad(\mathrm{in} / \mathrm{hr})\end{array}$ & $\begin{array}{c}\text { Post } \\
\text { Maintenance } \\
\text { Date }\end{array}$ & $\begin{array}{c}\text { Measured } \\
\text { Infiltration } \\
\text { Rate } \\
\text { (in/hr) } \\
\end{array}$ \\
\hline $1-\mathrm{A}$ & 2.5 & 0.5 & $8 / 24 / 2012$ & 5.6 & $10 / 5 / 2012$ & $1,190.6$ \\
\hline $3-\mathrm{A}$ & 20 & 0.5 & $8 / 24 / 2012$ & 27.3 & $10 / 5 / 2012$ & $1,779.9$ \\
\hline $3-\mathrm{B}$ & 20 & 4 & $8 / 24 / 2012$ & 94.0 & $10 / 5 / 2012$ & $1,670.2$ \\
\hline $3-\mathrm{C}$ & 20 & 7.5 & $8 / 24 / 2012$ & $1,263.1$ & $10 / 5 / 2012$ & $1,915.3$ \\
\hline 4-A & 40 & 0.5 & $8 / 24 / 2012$ & 12.3 & $10 / 5 / 2012$ & $1,236.5$ \\
\hline $5-\mathrm{A}$ & 75 & 0.5 & $8 / 24 / 2012$ & 43.5 & $10 / 5 / 2012$ & $1,004.0$ \\
\hline \multicolumn{7}{|c|}{ Control 19H } \\
\hline $1-\mathrm{A}$ & 2.5 & 0.5 & $8 / 24 / 2012$ & 12.1 & $10 / 5 / 2012$ & $1,474.5$ \\
\hline $3-\mathrm{A}$ & 20 & 0.5 & $8 / 24 / 2012$ & 17.3 & $10 / 5 / 2012$ & $1,340.0$ \\
\hline $3-\mathrm{B}$ & 20 & 4 & $8 / 24 / 2012$ & 510.7 & $10 / 5 / 2012$ & $2,073.0$ \\
\hline $3-\mathrm{C}$ & 20 & 7.5 & $8 / 24 / 2012$ & 987.2 & $10 / 5 / 2012$ & $1,444.3$ \\
\hline $4-\mathrm{A}$ & 40 & 0.5 & $8 / 24 / 2012$ & 55.5 & $10 / 5 / 2012$ & $1,067.9$ \\
\hline
\end{tabular}


Table 28 - Pre and Post Maintenance Surface Infiltration Test Results, Vac-Head Method

\begin{tabular}{|c|c|c|c|c|c|c|}
\hline \multicolumn{7}{|c|}{ Control 19G } \\
\hline $\begin{array}{c}\text { Test } \\
\text { Location }\end{array}$ & $X(f t)$ & $Y(f t)$ & $\begin{array}{c}\text { Pre- } \\
\text { Maintenance } \\
\text { Date }\end{array}$ & $\begin{array}{c}\text { Measured } \\
\text { Infiltration Rate } \\
\quad(\mathrm{in} / \mathrm{hr})\end{array}$ & $\begin{array}{c}\text { Post } \\
\text { Maintenance } \\
\text { Date }\end{array}$ & $\begin{array}{c}\text { Measured } \\
\text { Infiltration } \\
\text { Rate } \\
\text { (in/hr) }\end{array}$ \\
\hline $1-\mathrm{A}$ & 2.5 & 0.5 & $9 / 18 / 2013$ & 4.7 & $9 / 18 / 2013$ & $1,532.2$ \\
\hline $3-\mathrm{A}$ & 20 & 0.5 & $9 / 18 / 2013$ & 16.7 & $9 / 18 / 2013$ & $1,601.9$ \\
\hline 4-A & 40 & 0.5 & 9/18/2013 & 21.0 & 9/18/2013 & $1,601.9$ \\
\hline $5-\mathrm{A}$ & 75 & 0.5 & 9/18/2013 & 27.3 & 9/18/2013 & $1,762.1$ \\
\hline
\end{tabular}

To make a valid judgment over the effectiveness of different maintenance methods, statistical analysis is applied. Student's t-tests (paired two sample tests) are used to evaluate the significance of restoration of infiltration rates on clogged locations. The clogged locations on each control are selected based on the baseline infiltration rates. If any test location has an infiltration rate smaller than average baseline infiltration rates minus the standard deviation, is considered clogged and is included in paired t-tests. The unclogged locations that are excluded from t-test analyses, from Tables 24 to 27 , are shaded in gray. The results from the all Student's t-tests are summarized in Tables 29 and 30.

Table 29 - Paired Student's T-Tests for Pre and Post Maintenance Surface Infiltration Rates, 19G

\begin{tabular}{llllllll}
\hline \multirow{2}{*}{ Maintenance Method } & \multicolumn{3}{c}{ Pre-Maintenance } & \multicolumn{3}{c}{ Post-Maintenance } & \multirow{2}{*}{ P } \\
\cline { 2 - 7 } & $\mathbf{N}$ & $\begin{array}{l}\text { Mean } \\
\text { (in/hr) }\end{array}$ & $\begin{array}{l}\text { St. Dev. } \\
\text { (in/hr) }\end{array}$ & N & $\begin{array}{l}\text { Mean } \\
\text { (in/hr) }\end{array}$ & $\begin{array}{l}\text { St. Dev. } \\
\text { (in/hr) }\end{array}$ & \\
\hline Sweeper/Vacuum Truck & 6 & 429 & 463 & 6 & 813 & 594 & 0.018 \\
\hline Pressurized Airjet \#1 & 5 & 227 & 268 & 5 & 1210 & 648 & 0.034 \\
\hline Pressurized Airjet \#2 & 5 & 37 & 35 & 5 & 1376 & 332 & 0.001 \\
\hline Vac-Head Method & 4 & 17.4 & 9.5 & 4 & 1624.5 & 97.4 & 0.000 \\
\hline
\end{tabular}


Table 30 - Paired Student's T-Tests for Pre and Post Maintenance Surface Infiltration Rates, 19H

\begin{tabular}{llllllll}
\hline \multirow{2}{*}{ Maintenance Method } & \multicolumn{3}{c}{ Pre-Maintenance } & \multicolumn{3}{c}{ Post-Maintenance } & \multirow{2}{*}{\begin{tabular}{l} 
Mean \\
\cline { 2 - 7 }
\end{tabular}} \\
\cline { 2 - 7 } & $\mathbf{N}$ & $\begin{array}{l}\text { Mean } \\
\text { (in/hr) }\end{array}$ & $\begin{array}{l}\text { St. Dev. Dev. } \\
\text { (in/hr) }\end{array}$ & $\mathbf{N}$ & $\begin{array}{l}\text { St.hr) } \\
\text { (in/hr) }\end{array}$ & \\
\hline Pressurized Airjet \#1 & 7 & 241 & 205 & 7 & 1751 & 371 & 0.000 \\
\hline Pressurized Airjet \#2 & 5 & 317 & 429 & 5 & 1480 & 368 & 0.004 \\
\hline
\end{tabular}

Results from Tables 28 and 29 show that all surface maintenance treatments were able to restore the infiltration rates of tests location with a statistical significance of at least $95 \%$ level $(\alpha=0.05)$. While these results show that all used methods have resulted in significant differences between measured infiltration rates, to compare the effectiveness of all methods, the average infiltration rates measured before and after each maintenance treatment are plotted together in Figure 82. As it can be seen in this figure, the Sweeping/Vacuum method was the only method that were not able to restore the infiltration rates of control $19 \mathrm{G}$ to its initial rates. While the other two methods have been both effective is restoring the infiltration rates for the GI controls, the Vac-Head method seems to have been slightly more successful than Airjet. 


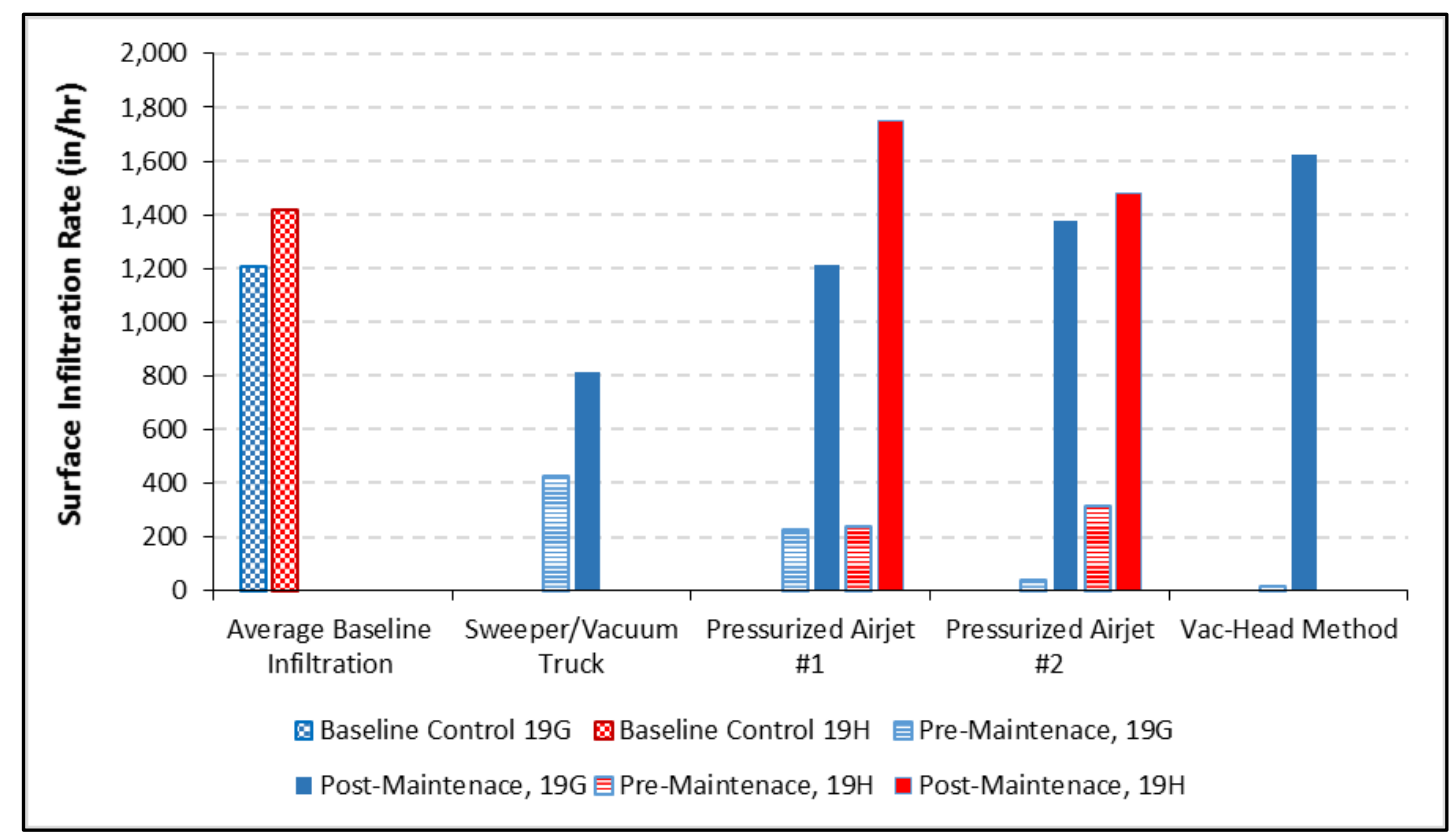

Figure 82 - Restoration of Average Surface Infiltration Rates for Different Maintenance Treatments

Figure 83 shows the restoration of infiltration rates from curbside locations of control 19G for the three different maintenance methods. The reason for selecting the curbside locations was their significant decreased infiltration rates and the concentration of runoff flow on these locations. Since both the first and second airjet methods have shown similar effectiveness only results from airjet \#1 is shown in this graph. The figure shows that only the Vac-Head method has been able to restore the infiltration rates for the most up-gradient location.

In conclusion, the analysis of measured surface infiltration rates from controls 19G and $19 \mathrm{H}$ indicate that airjet method and the Vac-Head method have been able to restore the short-term infiltration capacity of both controls, with the exception of the most up-gradient location for airjet method. To evaluate the long-term effectiveness of these two 
maintenance methods, the TDR data and the modeling results are used, which are explained in following sections.

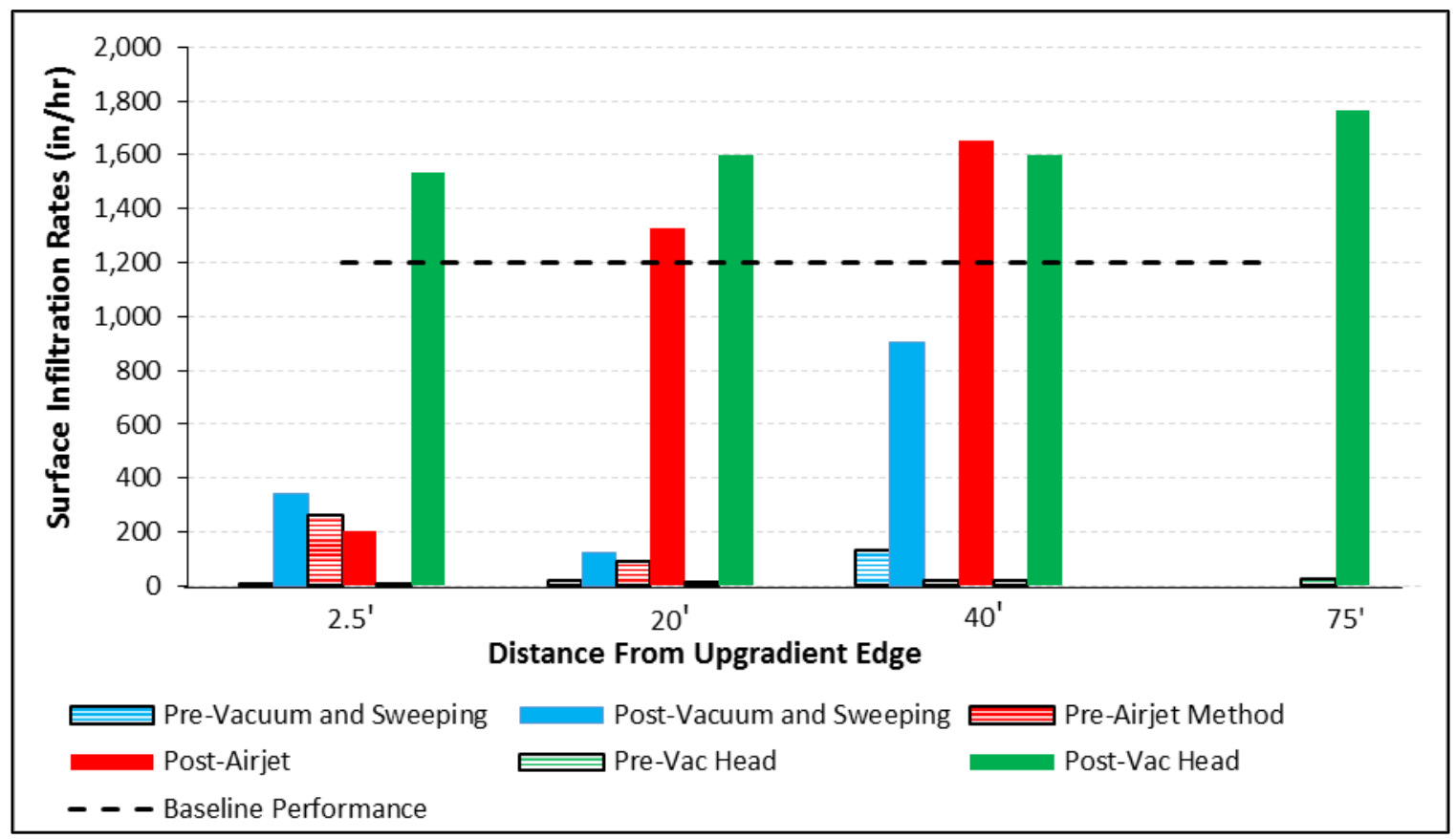

Figure 83 - Restoration of Surface Infiltration Rates for Curbside Locations along Control 19G and Different Maintenane Treatments

\subsubsection{TDR Data}

In Section 4.3, monitoring the clogging progression rate on the surface of control 19G initially after its construction was explained. The same method is applied to assess the surface clogging rates (based on the cumulative rainfall) and after each maintenance treatment. After the sweeping/vacuum and the airjet\#2, the curbside TDRS didn't record any VWC measurements greater than the previously defined thresholds $(0.1$ and 0.12 $\mathrm{cm}^{3} / \mathrm{cm}^{3}$ ). Figures 84 through 86 show the last measured TDRs responses greater than 0.1 $\mathrm{cm}^{3} / \mathrm{cm}^{3}$ threshold for the other three maintenance treatments. Since any location can be identified as "clogged" only once the corresponding TDR measurements drop below the 
defined threshold, only the last measured TDRs responses greater than the threshold limit are provided in these figures. Also to provide better comparison between the baseline clogging rate and the observed rate, the initial TDR responses after construction of GI controls are also included in each figure. It must be noted that the TDR installed at $2.5 \mathrm{ft}$ from upgradient edge never recorded any VWC measurement greater than $0.1 \mathrm{~cm} 3 / \mathrm{cm} 3$ after December 2011. This is either due to heavy clogging rate at that location or the malfunction of the sensor.

While the initial clogging rate at the surface of control 19G was found to be equal to $8.9 \mathrm{ft} /$ in (feet per an inch of rainfall), after each maintenance treatment this rate differed depending on long-term effectiveness of used method. Figures 84 and 85 show that the surface clogging rate increased with first and second airjet maintenance efforts to $15 \mathrm{ft} / \mathrm{in}$ and $56 \mathrm{ft} /$ in, respectively. On the other hand the Vac-Head method were able to recover the clogging rate to even a slower rate of $8 \mathrm{ft} / \mathrm{in}$. Although it must be noted again that as explained in Section 5.3, the modeling results indicated that following the construction of new GI controls in CSO130 basin in 2013, the drainage ratio of control 19G were approximately decreased by approximately $36 \%$ which explains the observation of a slower clogging rate. By assuming a direct linear relationship between clogging rate and drainage area, the clogging rate after the Vac-Head method is equal to $12.5 \mathrm{ft} /$ in which is still slower than the other two maintenance treatments. 


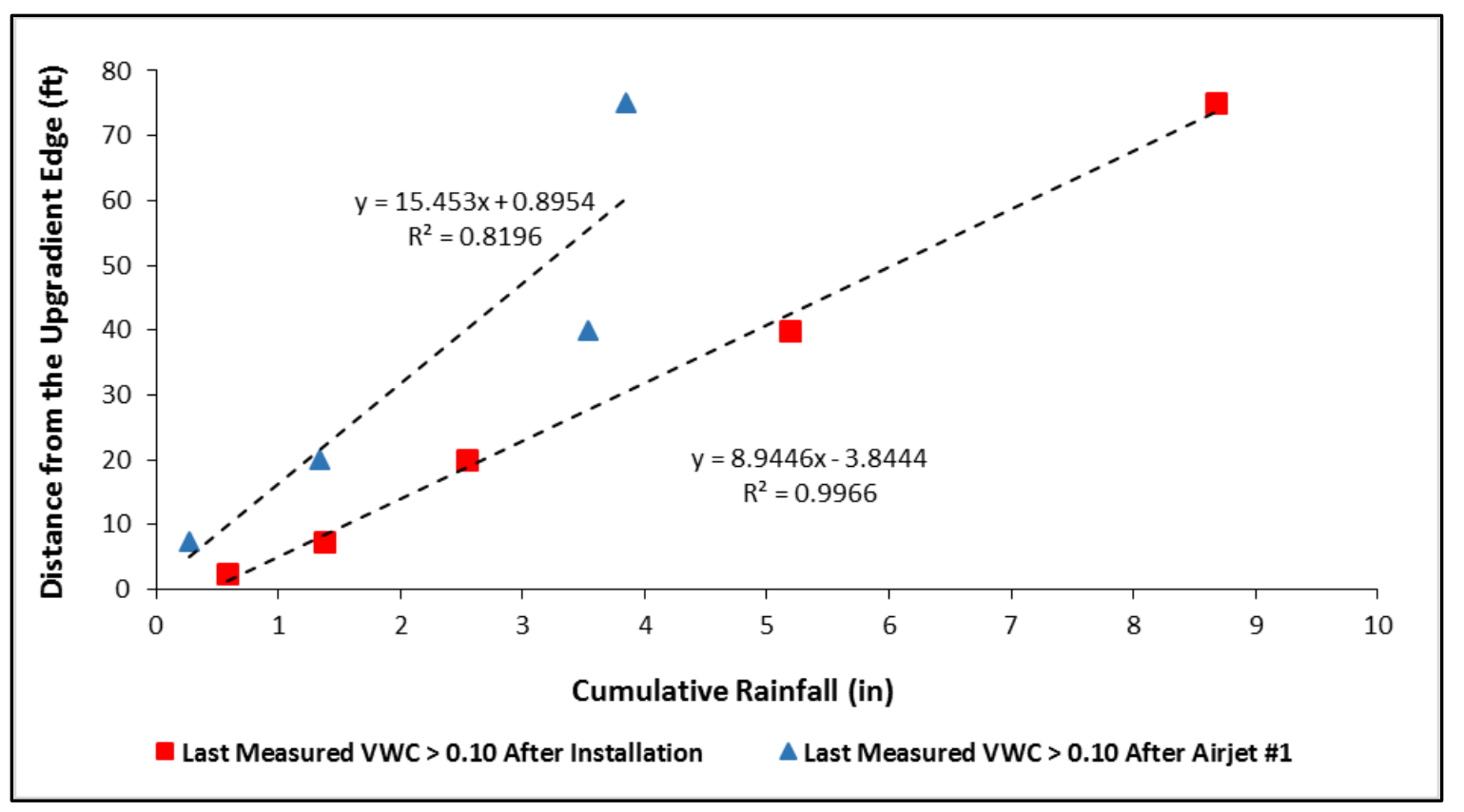

Figure 84 - Last Curbside TDRs' Measurements after Construction and Airjet \#1, Control 19G

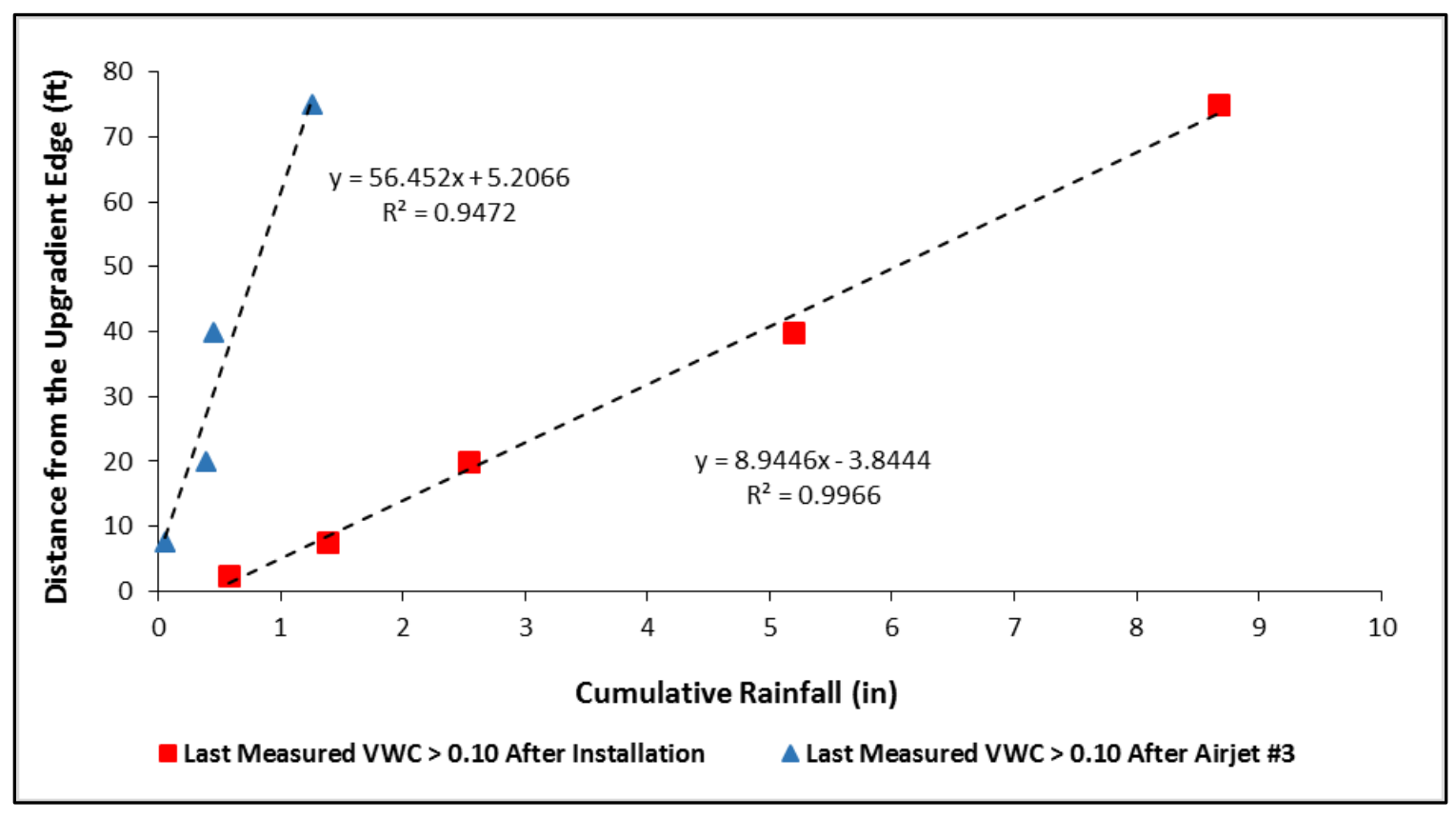

Figure 85 - Last Curbside TDRs' Measurements after Construction and Airjet \#3, Control 19G 


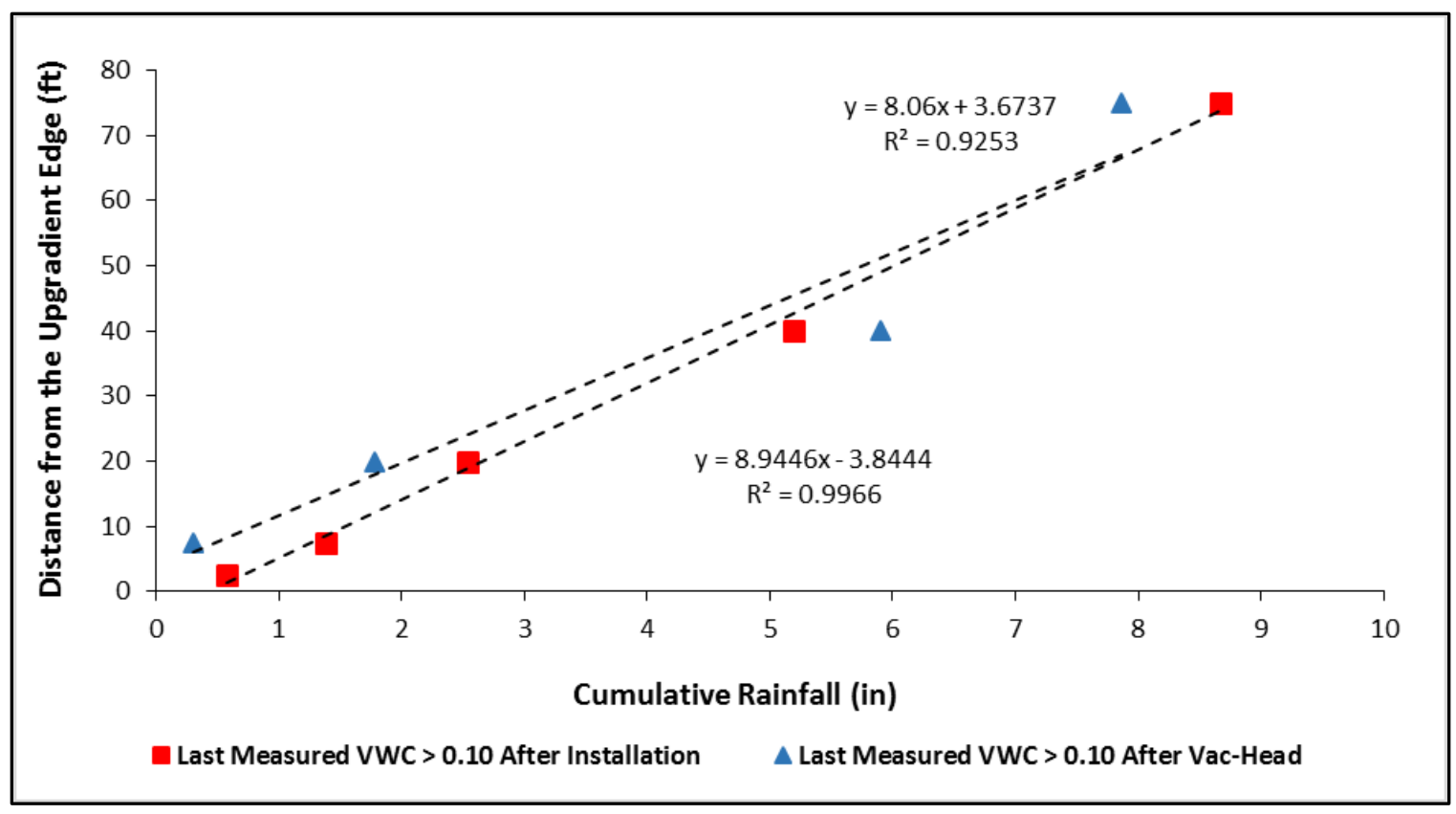

Figure 86 - Last Curbside TDRs' Measurements after Construction and Vac-Head, Control 19G

\subsubsection{Modeling Results}

Modeling of the water level inside the GI controls were explained in chapter 5 and it was mentioned that the developed models were used as assessment tools to compare the hydrological performance of the GI controls over their service lives with their initial performances. It was observed that once the clogging reaches the downgradient edge of the GI control, their infiltration capacities were decreased and were only recovered after conducting proper maintenance treatments. The changes in infiltration capacity of the GI controls, resulted from modeling technique 1, are shown in Figures 87 and 88. In these figures the time of maintenance treatments are marked with vertical dashed green lines. Also the cumulative rainfall depth following each maintenance treatment and before the infiltration capacity drops to lower than $90 \%$ are provided in Table 31 . Once the infiltration capacity drops to lower than $90 \%$ the GI controls are considered clogged. 


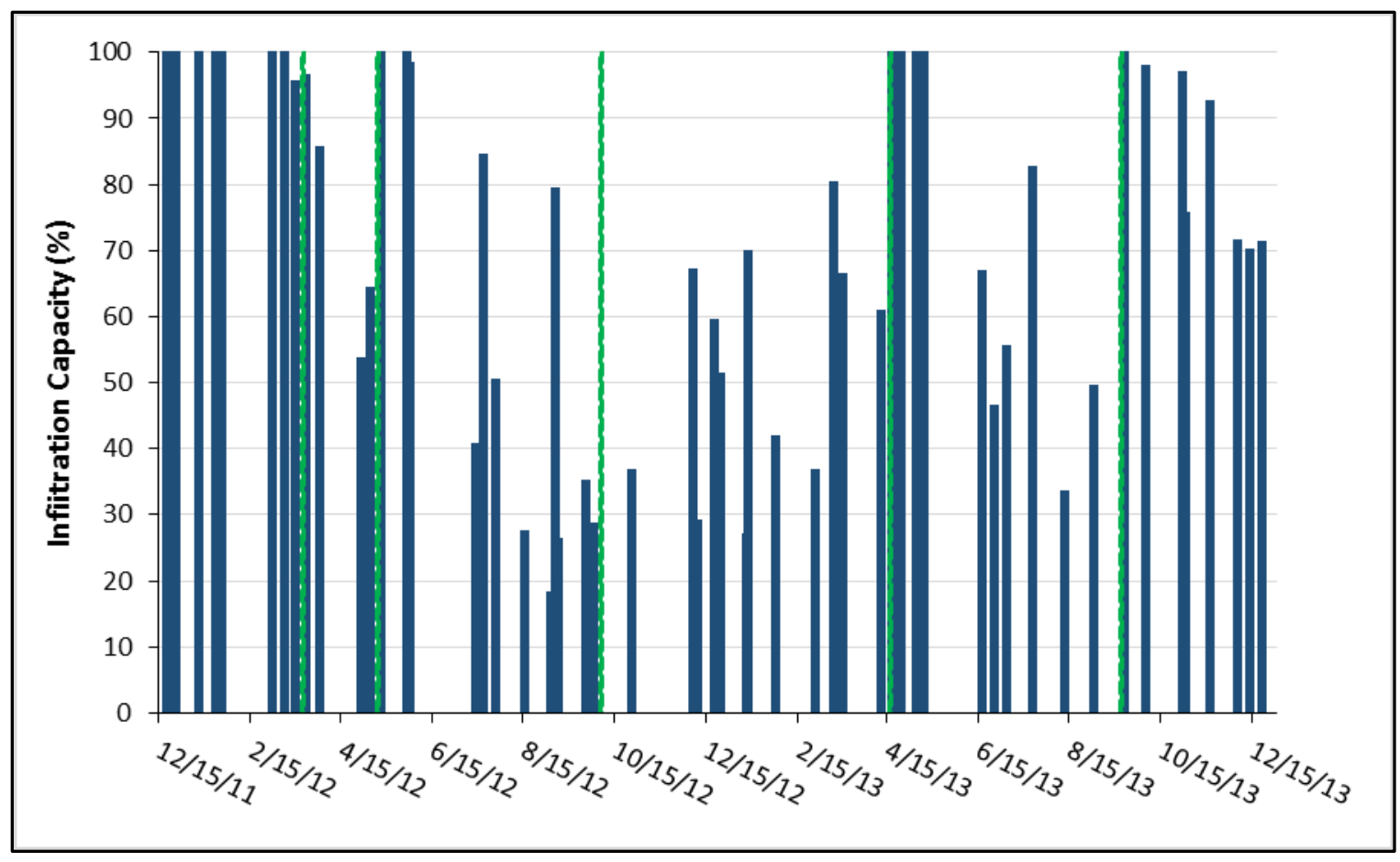

Figure 87 - Infiltration Capacity and Surface Maintenance Treatments, Control 19G

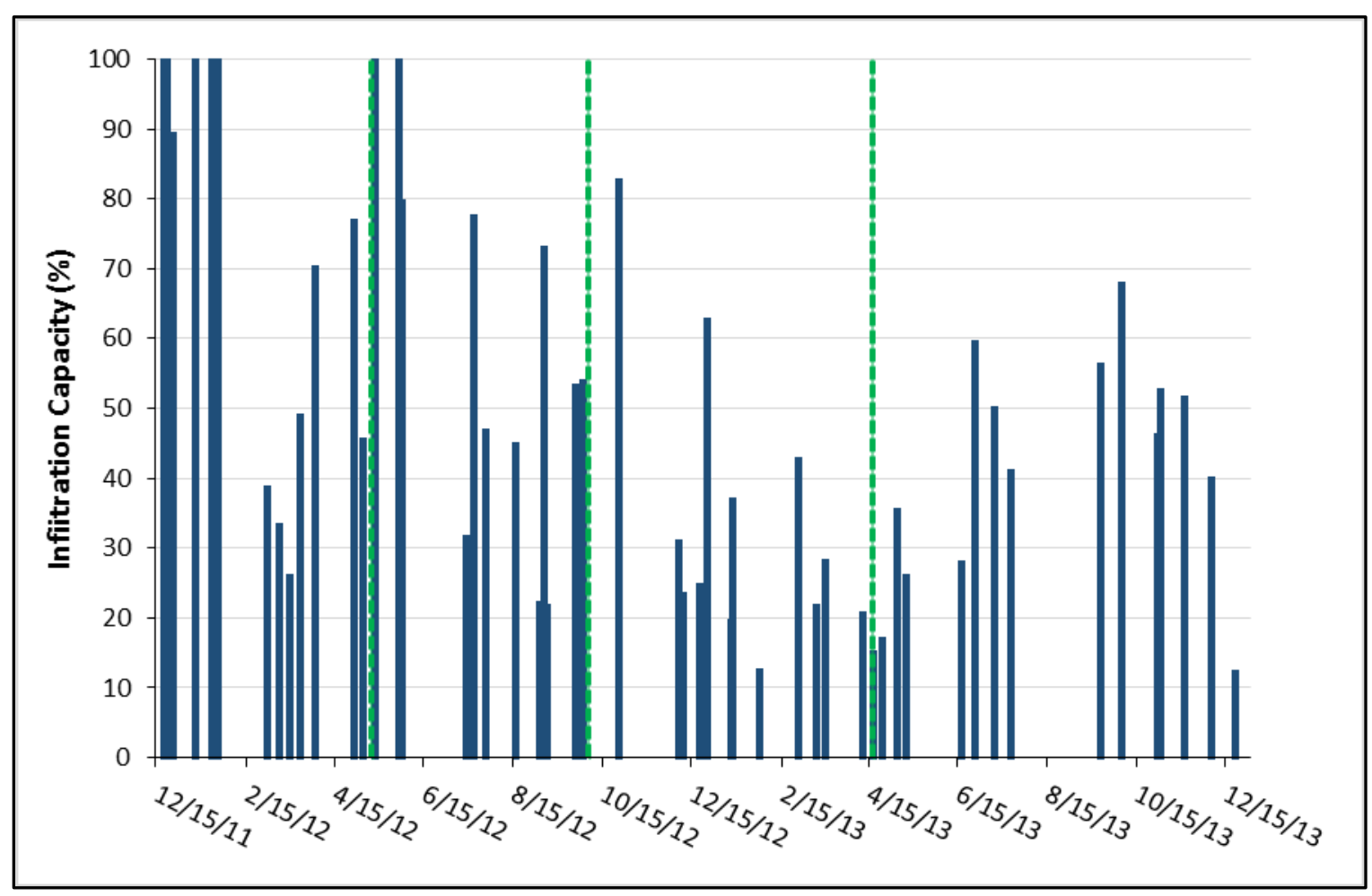

Figure 88 - Infiltration Capacity and Surface Maintenance Treatments, Control 19H 
Table 31 - Cumulative Rainfall Depth Effectively Captured after Each Maintenance Treatment

\begin{tabular}{clc}
\hline Control ID & $\begin{array}{l}\text { Surface Maintenance } \\
\text { Treatment }\end{array}$ & $\begin{array}{c}\text { Cumulative Rainfall Depth } \\
\text { before Infiltration Capacity } \\
\text { Decreases by }>90 \% \text { (inches) }\end{array}$ \\
\hline $19 \mathrm{G}$ & Installation & 11.14 \\
\hline $19 \mathrm{G}$ & Sweeper/Vacuum Truck & 1.63 \\
\hline $19 \mathrm{G}$ & Pressurized Airjet \#1 & 6.14 \\
\hline $19 \mathrm{G}$ & Pressurized Airjet \#2 & N/A \\
\hline $19 \mathrm{G}$ & Pressurized Airjet \#3 & 3.86 \\
\hline $19 \mathrm{G}$ & Vac-Head & 8.81 \\
\hline $19 \mathrm{H}$ & Installation & 6.78 \\
\hline $19 \mathrm{H}$ & Pressurized Airjet \#1 & 4.82 \\
\hline $19 \mathrm{H}$ & Pressurized Airjet \#2 & N/A* \\
\hline $19 \mathrm{H}$ & Pressurized Airjet \#3 & $\mathrm{N}^{*}$ \\
\hline
\end{tabular}

* No major (>0.5 inch) rainfall events were observed with an infiltration capacity greater than $90 \%$.

Results from these figures and Table 31 show that the first airjet maintenance and Vac-Head method have been the most effective maintenance efforts. The third airjet maintenance has been somewhat effective on control 19G. It should be mentioned once again that before the Vac-Head method the drainage area of control 19G is suspected to be decreased by $36 \%$. By considering this, the rainfall depth value in Table 31 can be corrected as: $(1-0.36) \times 8.81=5.64$ inches, which is similar to the value observed for the first airjet maintenance method.

\subsection{Clogging Material}

During the first three maintenance efforts, samples of the clogging materials were collected for further laboratory analysis. The samples were tested for size distribution analysis by use of sieve gradation according to ASTM D6913-04 and determination of organic matter according to ASTM2974-07a. The testing procedure was revised with each maintenance treatment. After the first maintenance, it was decided to collect the materials separately from down-gradient to up-gradient locations and by dividing the surface of the 
pavements into different sub-sections. Controls $19 \mathrm{G}$ and $19 \mathrm{H}$ were divided into four $30-\mathrm{ft}$ and two 27.5-ft segments respectively. The samples were labeled with subscripts A, B, C, and $\mathrm{D}$ in which $\mathrm{A}$ and $\mathrm{D}$ (B in case of control 19H) indicate the most upgradient and most downgradient locations, respectively. While organic content matter were only tested for sub-samples of the collected material during the second maintenance method, the plan was revised to test the organic content from sieve gradation to determine the size distribution of the organic content as well. The results from these tests are explained in the following sections.

\subsubsection{Size Distribution}

The sieve numbers used in this analysis were as follows: $0.312 ", \# 4, \# 10, \# 20, \# 40$, $\# 80$, \#100, and \#200. Because of the high percentage of organic matter hydrometer analysis couldn’t be performed for materials passing \#200 sieve. Size distribution analysis results are shown in Figures 89 through 93. Having the particle size distribution plots, the coefficients of uniformity $(\mathrm{Cu})$ and curvature $(\mathrm{Cc})$ are calculated for each sample and provided in Table 32 .

Since all samples showed more than 50\% passing sieve \#4 (4.75 mm) and less than 50\% passing sieve \#200 (0.075 mm), they are classified as "Sand" according to Unified Soil Classification System. For a sand sample to be classified as "well graded" it should have a $\mathrm{Cu} \geq 6$ and $1<\mathrm{Cc}<3$. With this criteria except for three samples that are shown in bold in Table 31, the rest are classified as "poorly graded". 


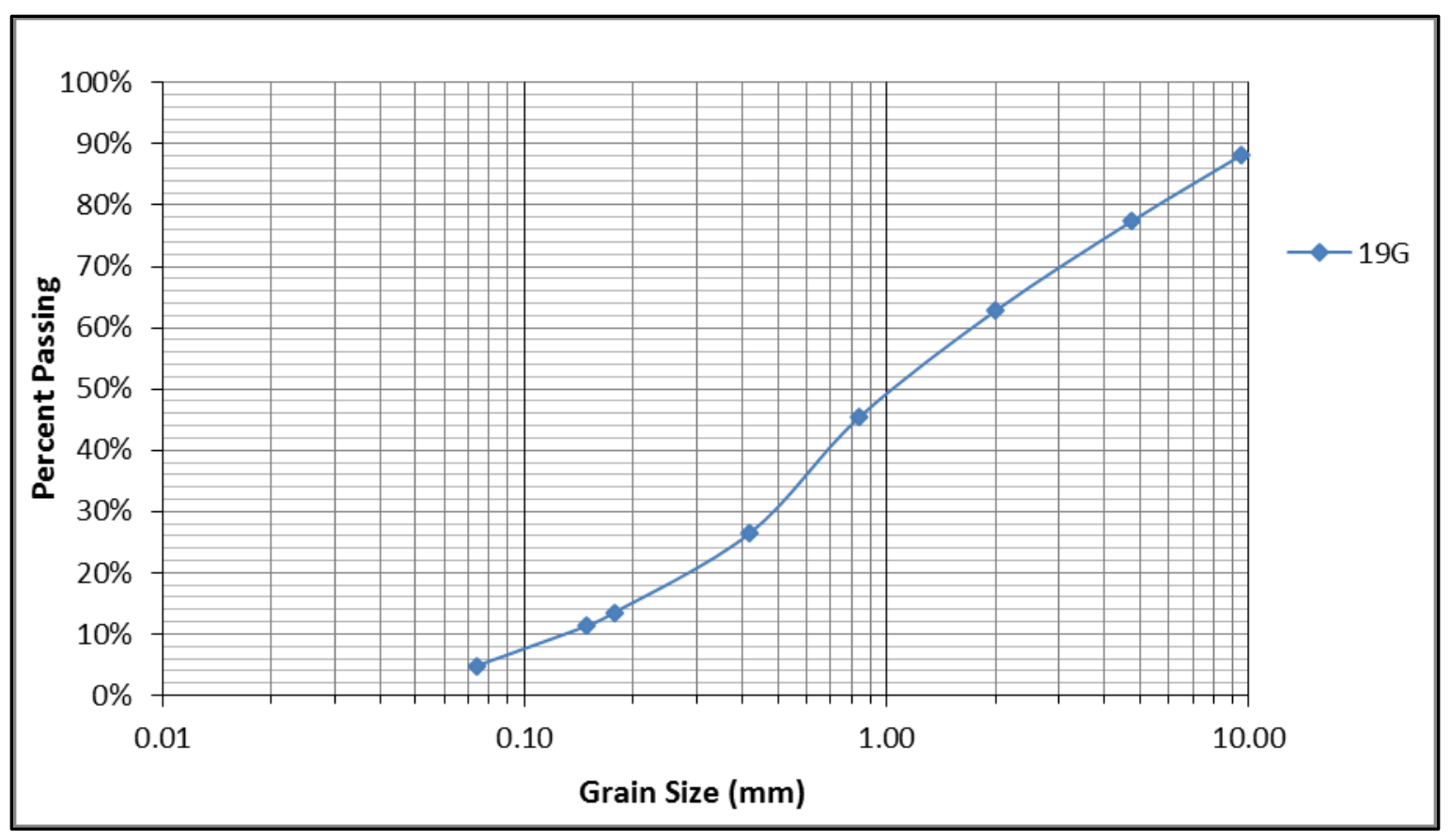

Figure 89 - Size Distribution of Collected Samples from Vacuum and Sweeping Maintenance,

Control 19G

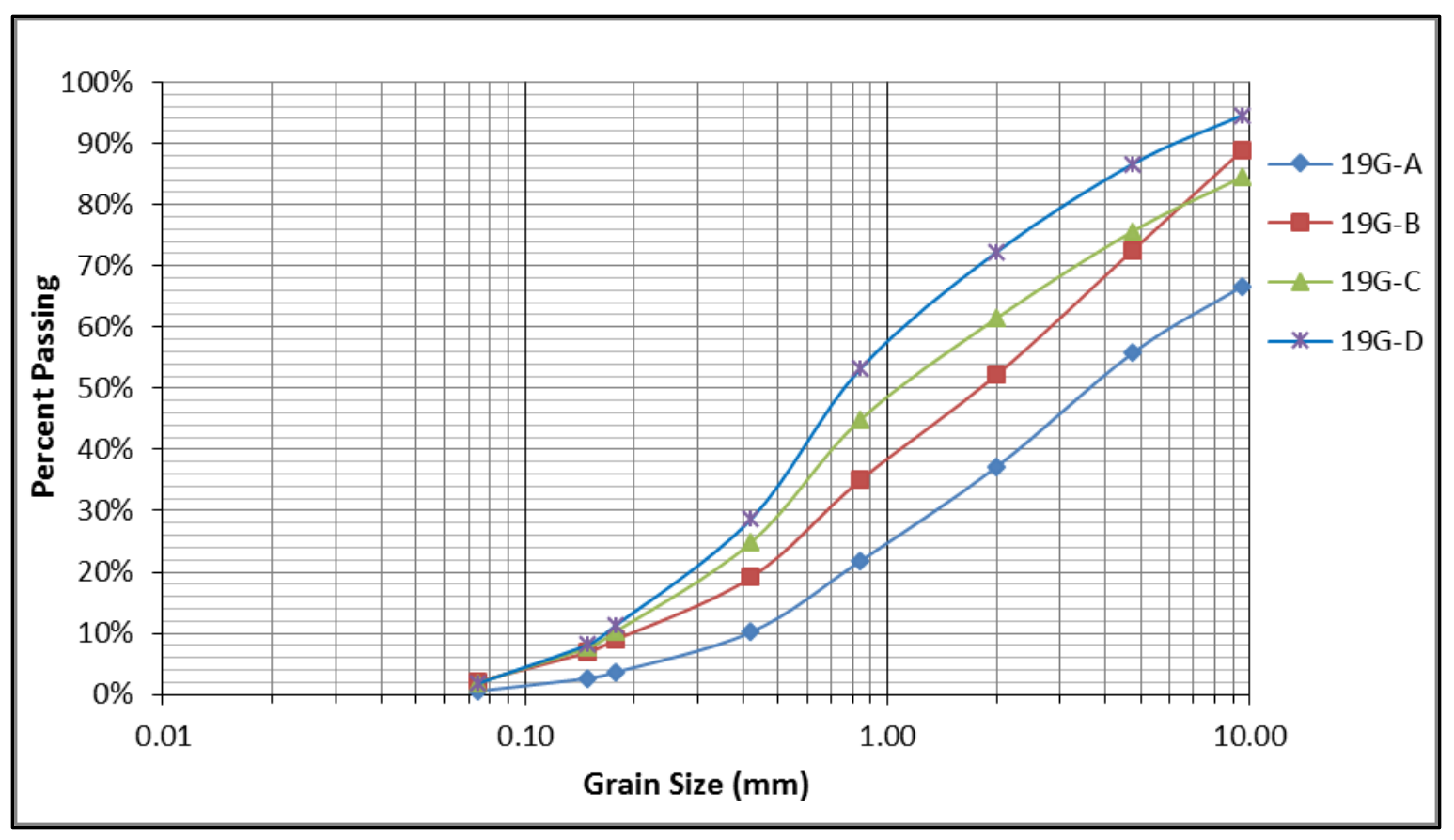

Figure 90 - Size Distribution of Collected Samples from Airjet \#1 Maintenance, Control 19G 


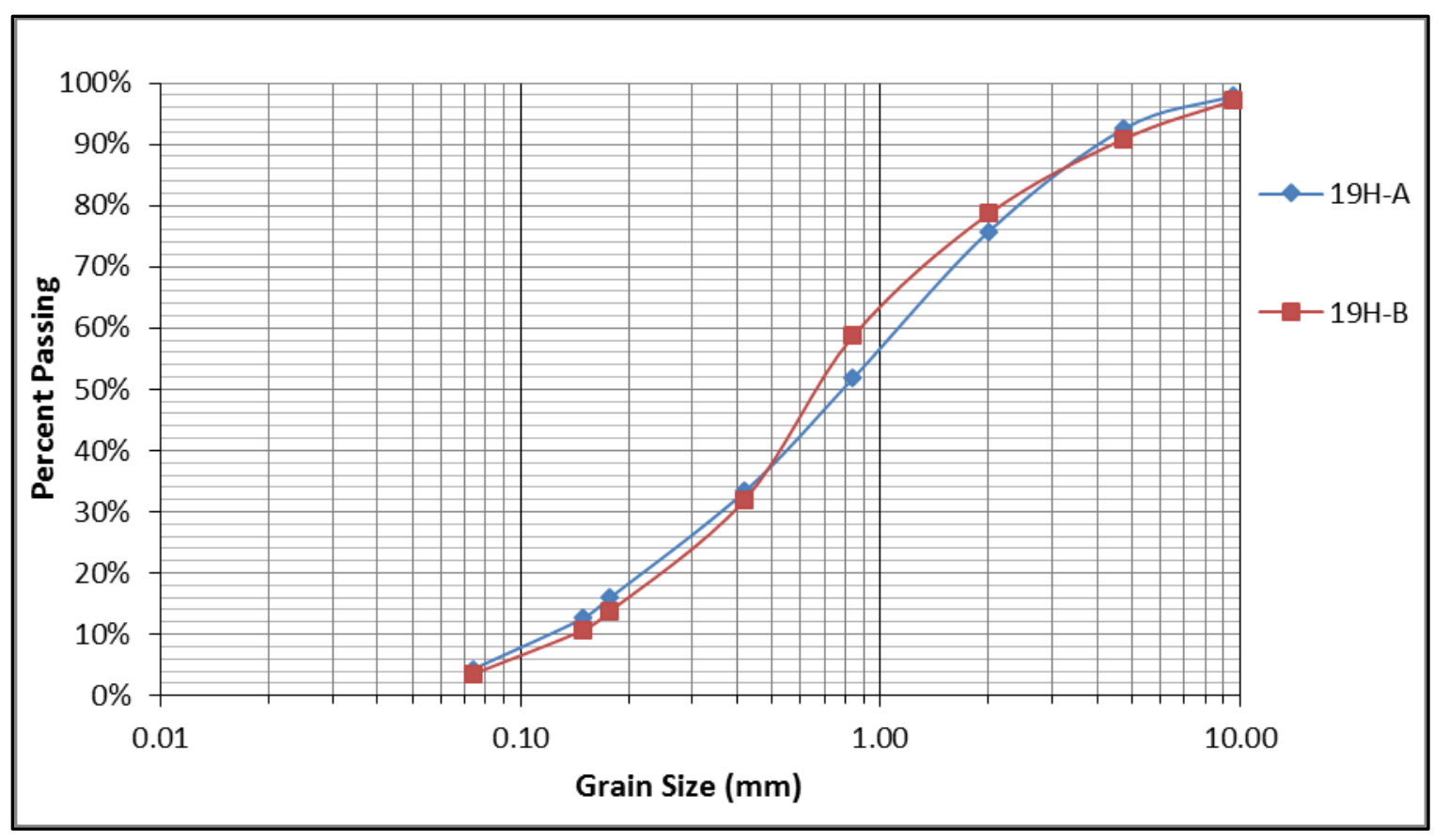

Figure 91 - Size Distribution of Collected Samples from Airjet \#1 Maintenance, Control 19H

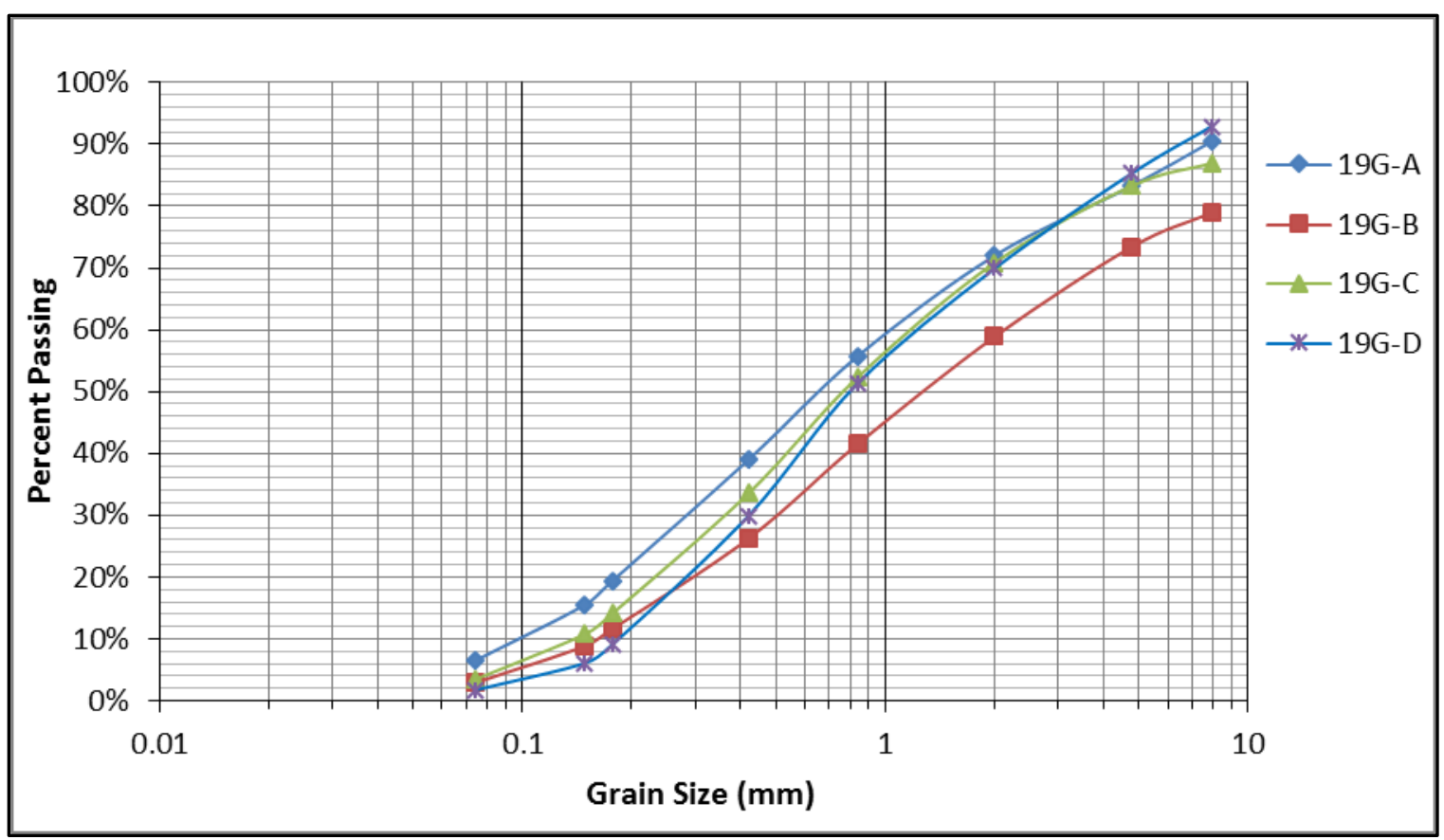

Figure 92 - Size Distribution of Collected Samples from Airjet \#2 Maintenance, Control 19G 


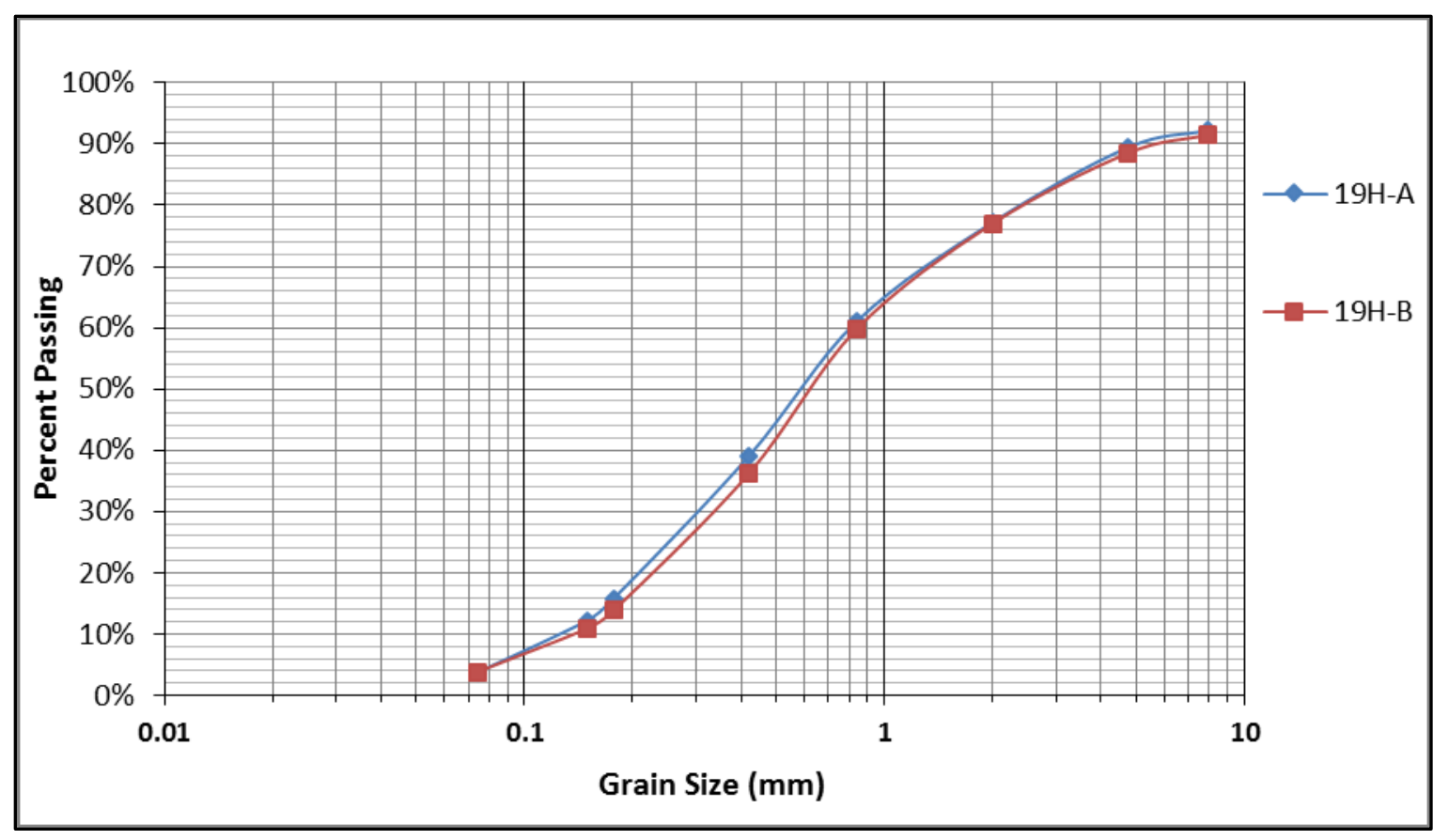

Figure 93 - Size Distribution of Collected Samples from Airjet \#2 Maintenance, Control 19H

Table 32 - Coefficients of Uniformity and Curvature from Size Distribution Analysis

\begin{tabular}{cccc}
\hline Maintenance Method & $\begin{array}{c}\text { Control and } \\
\text { Sample ID }\end{array}$ & Cu & Cc \\
\hline Vacuum / Sweeping & 19G & $\mathbf{1 3 . 8 5}$ & $\mathbf{1 . 0 7}$ \\
\hline Airjet \#1 & 19G - A & 14.29 & 0.67 \\
\hline Airjet \#1 & 19G - B & 14.00 & 0.83 \\
\hline Airjet \#1 & 19G - C & 10.59 & 0.82 \\
\hline Airjet \#1 & 19G - D & $\mathbf{7 . 5 0}$ & $\mathbf{1 . 0 1}$ \\
\hline Airjet \#1 & 19H - A & 10.91 & 0.93 \\
\hline Airjet \#1 & 19H - B & $\mathbf{6 . 4 3}$ & $\mathbf{1 . 2 7}$ \\
\hline Airjet \#2 & 19G - A & 11.34 & 0.73 \\
\hline Airjet \#2 & 19G - B & 12.94 & 0.67 \\
\hline Airjet \#2 & 19G - C & 8.00 & 0.72 \\
\hline Airjet \#2 & 19G - D & 7.50 & 0.76 \\
\hline Airjet \#2 & 19H - A & 6.15 & 0.89 \\
\hline Airjet \#2 & 19H - B & 6.07 & 0.97 \\
\hline
\end{tabular}




\subsubsection{Organic Content}

To determine the percentage of organic content, selected sub samples were first dried for 24 hours in the oven at 105 Celsius. In the next step the samples were burnt in a high temperature oven at 440 Celsius for another 24 hours. The difference in the weight of the sample before and after burning is used to calculate the percentage of organic matter. The results of organic content tests for the first two maintenance methods are provided in Table 33.

Table 33 - Organic Content Test Results for the First Two Maintenance Treatments

\begin{tabular}{cccc}
\hline Maintenance Method & $\begin{array}{c}\text { Control and } \\
\text { Sample ID }\end{array}$ & $\begin{array}{c}\text { Organic Content } \\
(\mathbf{\%})\end{array}$ & $\begin{array}{c}\text { Mean Organic } \\
\text { Content (\%) }\end{array}$ \\
\hline Vacuum / Sweeping & 19G & 8.03 & \\
\hline Airjet \#1 & 19G - A & 47.11 & \multirow{2}{*}{26.65} \\
\hline Airjet \#1 & 19G - B & 20.88 & \\
\hline Airjet \#1 & 19G - C & 20.99 & \multirow{2}{*}{15.15} \\
\hline Airjet \#1 & 19G - D & 17.60 & \\
\hline Airjet \#1 & 19H - A & 18.75 & \\
\hline Airjet \#1 & 19H - B & 11.54 &
\end{tabular}

It should be mentioned that testing on collected samples from the Vacuum and Sweeping were conducted 65 days after the maintenance treatment and it is suspected that some of the organic matter were already decomposed. For the second and third maintenance treatments all tests were conducted in a 2 -week period after the collection of samples. To better analyze the distribution of organic content from the third maintenance treatment, the samples were selected from the remaining material of the size distribution analysis. The results from these tests are provided in Table 34. For these test results two mean values are calculated: one for all samples from each section of the GI control 
remaining on different sieves and one for all samples in the GI control and remaining on one sieve only.

Table 34 - Size Gradation of Organic Content for Third Maintenance Treatment (Airjet \#2)

\begin{tabular}{ccccccccc}
\hline $\begin{array}{c}\text { Sieve Opening } \\
(\mathbf{m m})\end{array}$ & \multicolumn{8}{c}{ Percentage of Organic Content (Weight Basis) } \\
\cline { 2 - 9 } & 19G- A & 19G - B & 19G - C & 19G - D & Mean & 19H - A & 19H - B & Mean \\
\hline 7.92 & 36.24 & 15.81 & 28.10 & 20.84 & 25.25 & 44.42 & 34.07 & 39.24 \\
\hline 4.76 & 48.09 & 41.53 & 37.45 & 32.40 & 39.87 & 37.95 & 18.62 & 28.28 \\
\hline 2 & 60.70 & 72.35 & 58.12 & 41.39 & 58.14 & 30.40 & 33.80 & 32.10 \\
\hline 0.84 & 73.12 & 57.84 & 59.23 & 42.96 & 58.28 & 32.33 & 51.26 & 41.80 \\
\hline 0.42 & 28.47 & 26.35 & 18.38 & 12.58 & 21.45 & 24.59 & 12.47 & 18.53 \\
\hline 0.177 & 18.74 & 18.04 & 9.86 & 6.40 & 13.26 & 35.59 & 7.45 & 21.52 \\
\hline 0.149 & 19.66 & 17.32 & 11.01 & 9.75 & 14.43 & 19.27 & 9.32 & 14.29 \\
\hline 0.074 & 15.93 & 14.20 & 11.95 & 8.96 & 12.76 & 9.73 & 8.65 & 9.19 \\
\hline Pan & 12.07 & 8.34 & 11.72 & 8.74 & 10.22 & 9.85 & 8.23 & 9.04 \\
\hline Mean & 34.78 & 30.20 & 27.31 & 20.45 & 28.18 & 27.13 & 20.43 & 23.78 \\
\hline
\end{tabular}

Results from Tables 32 and 33 show that both GI controls had an average of 15 to 28 percent of organic content clogging their surfaces and collected samples from both airjet maintenances show that control 19G had slightly more organic content trapped between its gaps than control 19H. Table 34 show that for both GI controls the percentage of recovered organic material are greater for downgradient sections compared to upgradient areas. This corresponds to the low density of organic materials that allows them to flow with runoff to more downgradient sections while the inorganic finer sediments settle down quicker, due to their high density, and are subsequently trapped in more upgradient sections. 


\section{OVERALL EEFFECTIVENESS OF GI STORMWATER CONTROLS}

As previously discussed, 18 permeable pavement strips and 29 treeboxes were designed for installation in CSO130 to mitigate the stormwater overflows from this sewershed. Previous sections of this document were focused on evaluating the individual hydrological performance of the first two permeable pavements which were constructed in December 2011. The remaining proposed GI controls were installed in late 2012 and early 2013. This chapter briefly compares the overflow volumes from CSO130 sewershed before and after 2012-2013 construction. A regression analysis is carried out to identify the parameters affecting the CSO volumes in CSO130 which are then used to set up a regression equation to model the overflow volumes. The model is then utilized as an assessment tool to evaluate the effectiveness of installed GI controls in CSO mitigation.

As previously mentioned, the MSD's objective was to reduce the number of overflows in CSO 130 from 16 to 8, for the typical rainfall year (year 2001). Figure 94 shows the rainfall events for year 2001 which are identified with the assumption of a 6hour dry period before each event. By assuming a direct relationship between rain depth and overflow volumes, all rain events equal to or smaller than 1.09 inches should be captured to achieve MSD's defined objective. 


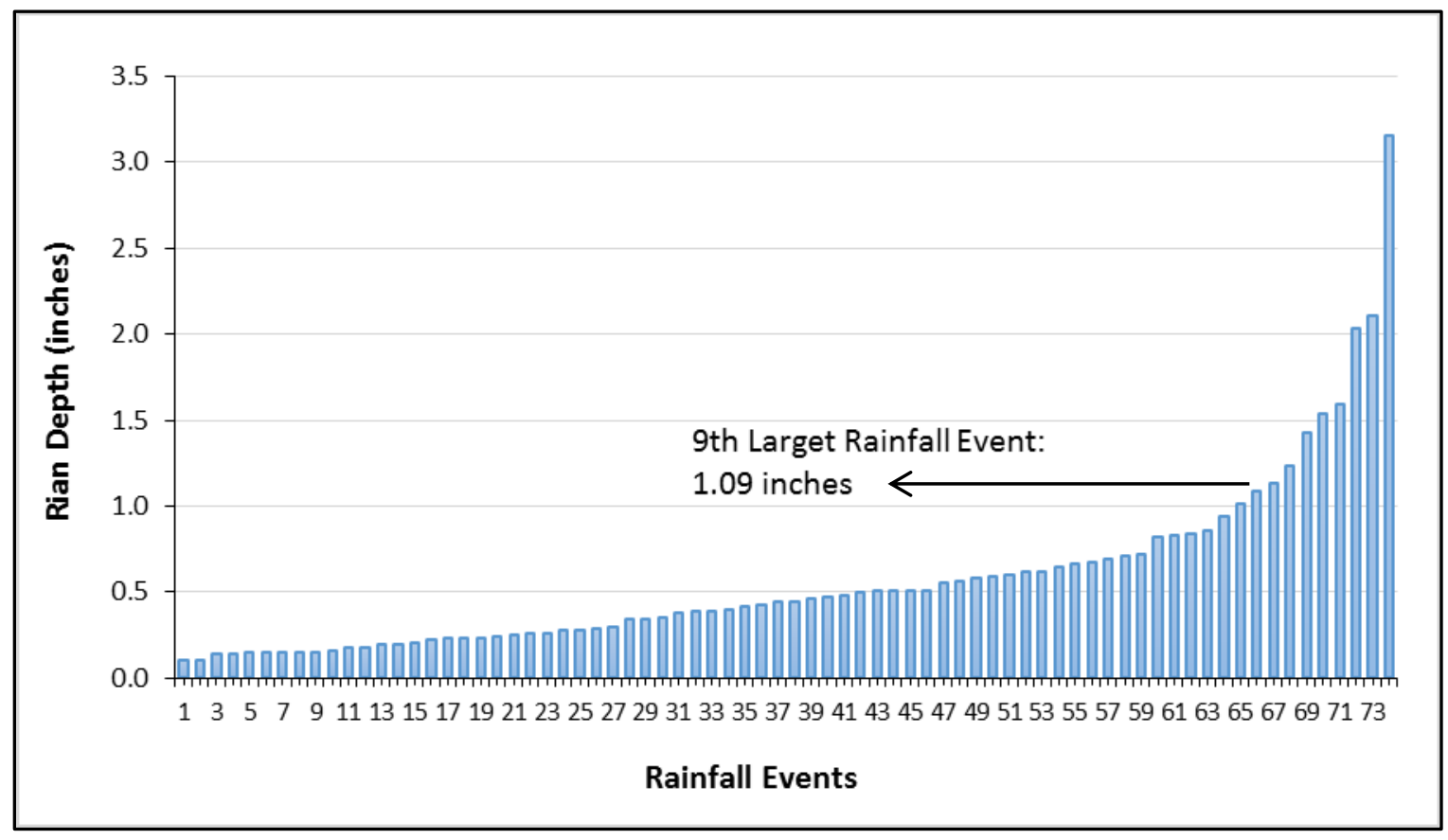

Figure 94 - Typical Year Rainfall Distribution using Year 2001 Rainfall Data

To investigate the relationship between rain depths and overflow volumes, rain depths values before the $2^{\text {nd }}$ phase of construction (December 2011-August 2012) are plotted versus the measured overflows with a $2^{\text {nd }}$ order polynomial trend line shown in Figure 95. As it can be seen, the R-Squared value is approximately 0.8. Although a direct relationship between overflow volumes and rain depth values can be observed in Figure 95, the relatively low value of correlation coefficient suggests involvement of other factors contributing to the overflow volumes (e.g. rainfall intensities, rainfall durations, and dry periods between overflow events). To investigate the effects of these other factors, a multivariant regression model was established which is explained in the following section. 


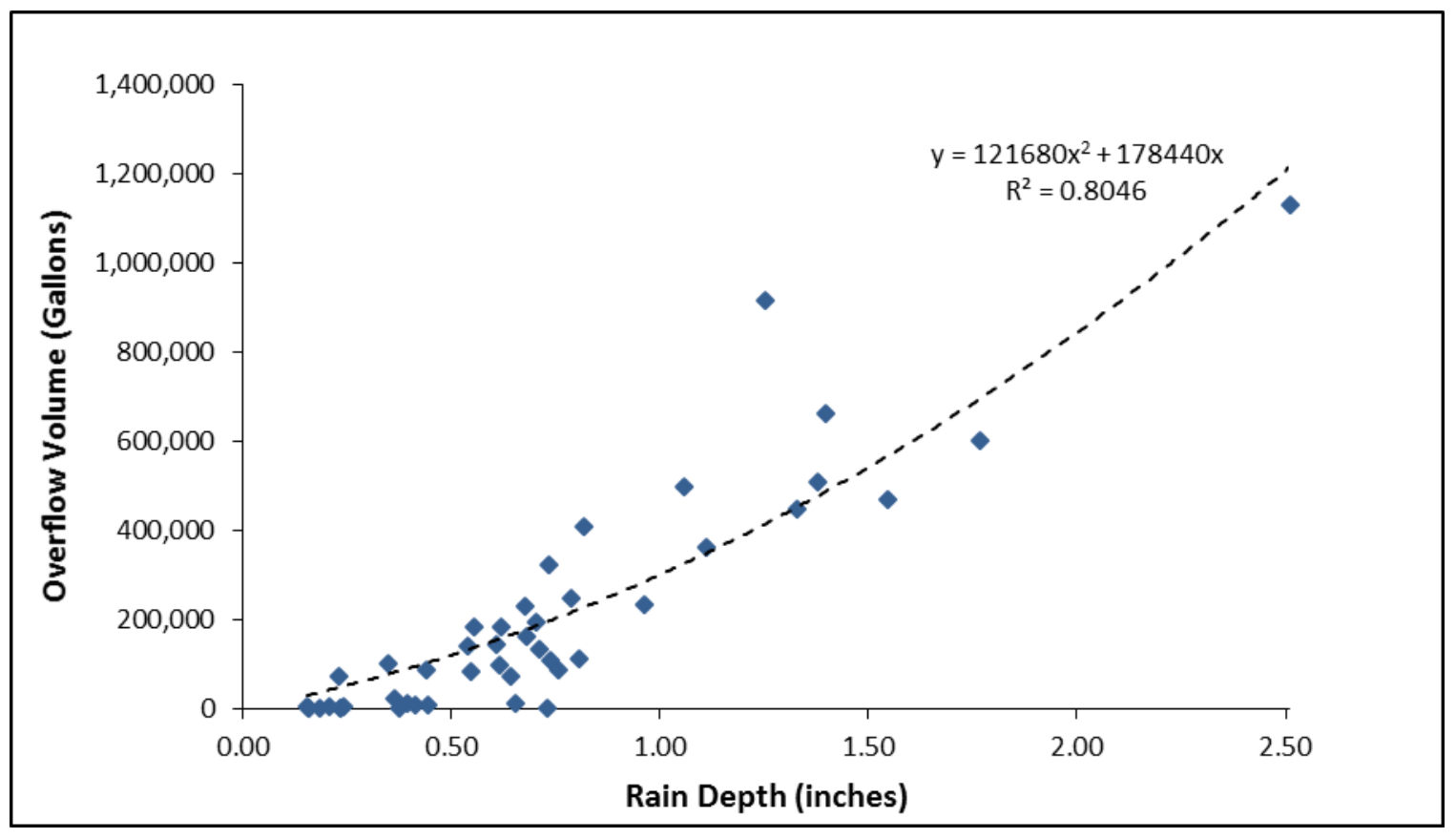

Figure 95 - Rain Depth versus Overflow Volume, CSO130

\subsubsection{Modeling the Overflows of CSO130}

A regression analysis was carried out to model the overflow volumes from CSO130 for rainfall events prior to construction of new GI controls. Regression analysis can be utilized to ascertain the causal effects of suspected factors on the response of the problem. In this regression analysis, the suspecting factors affecting the overflow volumes from CSO130 are selected and their relationship and effects on the resultant intrinsic permeability values are investigated. The input parameters entered into the model are defined in Table 14, and the output was defined as the observed overflow volume (gallons). The regression model was developed by using the statistical software package Minitab (version 17). 
Table 35 - Parameters Selected for Regression Analysis of Overflow Volumes

\begin{tabular}{|c|c|c|c|c|c|c|}
\hline & & & Predictor & & & Response \\
\hline Type & $\begin{array}{l}\text { Rainfall } \\
\text { Depth }\end{array}$ & $\begin{array}{l}\text { Rainfall } \\
\text { Event } \\
\text { Duration }\end{array}$ & $\begin{array}{l}\text { Rainfall } \\
\text { Event } \\
\text { Frequency }\end{array}$ & $\begin{array}{c}\text { Maximum } \\
\text { Rainfall } \\
\text { Intensity }\end{array}$ & $\begin{array}{c}\text { Antecedent } \\
\text { Rainfall } \\
\text { Depth }\end{array}$ & $\begin{array}{l}\text { Overflow } \\
\text { volume }\end{array}$ \\
\hline Unit & inches & hours & year & in $/ \mathrm{hr}$ & in & gallons \\
\hline
\end{tabular}

The regression model study utilized a step-wise process where critical parameters were first identified and then their influence determined. This is accomplished by entering the suspected factors and the response (overflow volumes in our case) values into individual columns of a spreadsheet. Then by selecting regression, the spreadsheet columns corresponding to the factors and response are defined for the software. The software determines the best relationship between factors that estimates the closest values to the response and reports the regression equation. Also by providing the P-values for each factor, Mintab reports the statistical significance of that factor in affecting the predicted response. Existence of lower $\mathrm{P}$-values indicates greater significance of that factor, e.g. $\mathrm{P}=$ 0.05 shows that the response has a significant effect on $95 \%$ of the predicted response values.

Residuals are the difference between the observed response values and the values that the model predicts. The distribution of residuals should not exhibit a discernible pattern otherwise the model is determined as "not a good fit". We can gain insight into the "goodness" of a fit by visually examining a plot of the residuals. If the residual plot has a pattern (that is, residual data points do not appear to have a random scatter), it would indicate that the model does not properly fit the data. 
Along with the regression equation, the software reports four plots for the residuals, which are: Histogram of Residuals, Normal Plot of Residuals, Residuals Versus Fit, and Residuals Versus Order Plot. These plots are used to determine if the regression is applicable or not. The residuals from an applicable regressions should follow a normal distribution around the regression which is tested by the Histogram plot of residuals. The residuals for a good fit also should fall in a straight line cantered at zero, shown by the normal probability plot. The plot of residuals versus the fitted values are used to make sure the residuals have a constant variance (exhibit homoscedasticity). Eventually the residuals versus the observed order show if the residuals are independent of each other (desirable) or there is any serial correlation (not desirable).

Another measure of goodness of a fit is the coefficient of determination, or $\mathrm{R}^{2}$ or R-squared. This coefficient indicates how closely values obtained from fitting a model match the response that the model is intended to predict. Closer values of $\mathrm{R}^{2}$ to 1 , indicates a good fitness of regression model. $\mathrm{R}^{2}$ is defined by using the residual variance from a fitted model:

$$
\mathrm{R}^{2}=1-\mathrm{SS}_{\text {resid }} / \mathrm{SS}_{\text {total }}
$$

Where:

$\mathrm{SS}_{\text {resid }}=$ the sum of the squared residuals from the regression .

$\mathrm{SS}_{\text {total }}=$ the sum of the squared differences from the mean of the response value 
It must be noted that since the maximum intensity and antecedent rainfall depth values can be estimated with different definitions, four intensity values and four antecedent rainfall depth values are calculated. The maximum intensities are calculated for 5-minute, 15-minute, 30-minute, and 60-minute periods during each rainfall event. Antecedent rainfall depths values are calculated for 1-day, 3-day, 5-day and 7-day before the start of each rainfall event. First to determine which is the best antecedent conditions to use, a regression is conducted to between the calculated antecedent values and by having the overflow values as the response. The Minitab output results of general regression analysis are as follows:

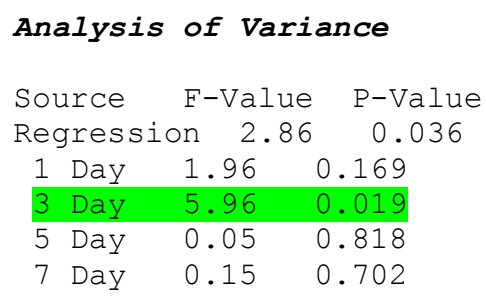

The results from this analysis indicates that 3-day antecedent rainfall depth (highlighted in green) is the most significant factor $(\mathrm{P}<0.05)$ affecting the overflow values. To determine the suitable intensity values to include in the analysis, similar effort is repeated but for calculated maximum intensity values. The results are as followed:

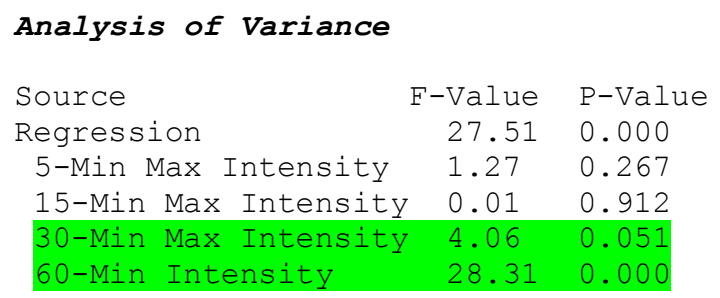

The results indicate the significance of 60-Minute intensity values over the other three values. Since the P value for 30-Min intensity is only very slightly greater than 0.05 , it is also selected for the next step of regression analysis. In the final step, the selected 
intensity and antecedent condition values along with rainfall depth, storm duration, and frequency values are analyzed with Minitab. Since the relationship of second order of rain depth and overflow volumes are suspected (see Figure 95), the parameters that represent the rain depth (rainfall depth and 3-day antecedent) are tested with both first and second order. Different combinations of these parameters are selected and the regression steps are repeated several times until the parameters with significant effects on the overflow volumes are identified. The results indicated that storm duration and first orders of rainfall depth and 3-day antecedent depth do not significantly affect the values of overflow volumes. The output of analysis results for the final derived regression model, which showed highest Rsquared values, are as follows:

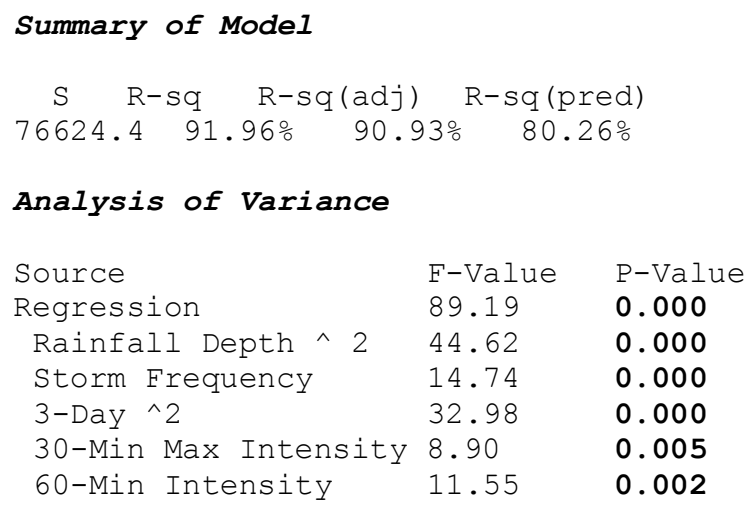

Figure 96 shows the residual plots for the final selected regression model which are satisfactory. The normal probability plot and the histogram of the residuals both indicate that the residuals have a normal distribution, and the plot of residuals versus the fitted values show homoscedasticity of the residuals. The plot showing the residuals versus the observed order indicate that the residuals are independent of each other and show no significant serial correlation. The Normal Probability plot in Figure 96 shows that the 
residuals fairly fall in a straight line centered at zero which is desirable. The final regression equation is:

Overflow Volume (Gallons) $=-30549+\left[175838\right.$ (Rainfall Depth (in)) $\left.{ }^{\wedge}\right]$

- [99939 Storm Frequency] + [70578 (3-Day Antecedent Rainfall Depth (in)) ^2] - [535638 (Max 30-Min Intensity (in/hr))] + [1190953 (Max 60-Min Intensity (in/hr))]

It must be noted that since all rainfall events used in this regression analysis were smaller than 2.5 inches, care must be given before applying the regression equation to rainfall events greater than 2.5 inches. The observed and modeled overflow volumes versus the rain depth values for their associated rainfall events are shown in Figure 97.

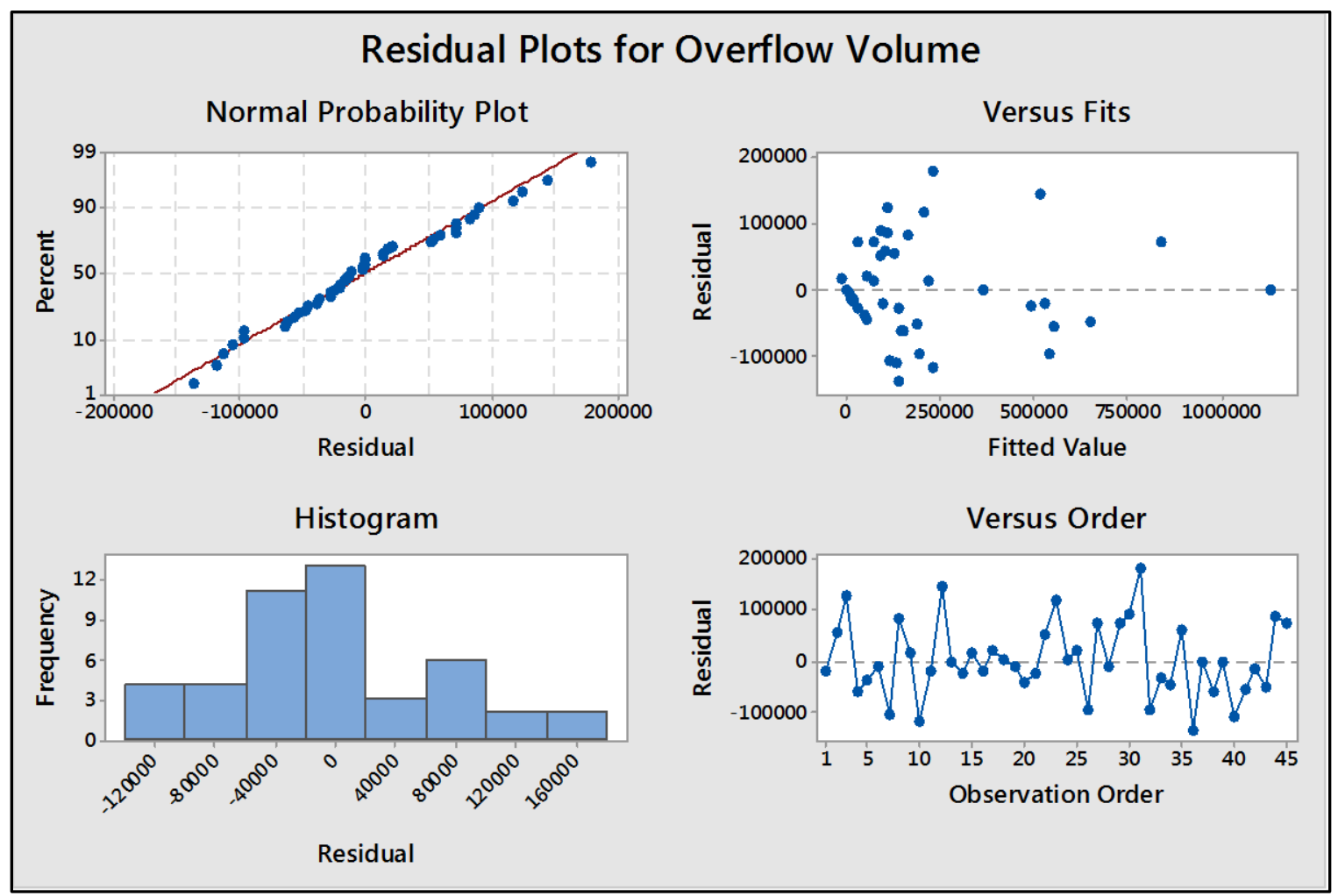

Figure 96 - Residual Plots for the Final Selected Regression Model 


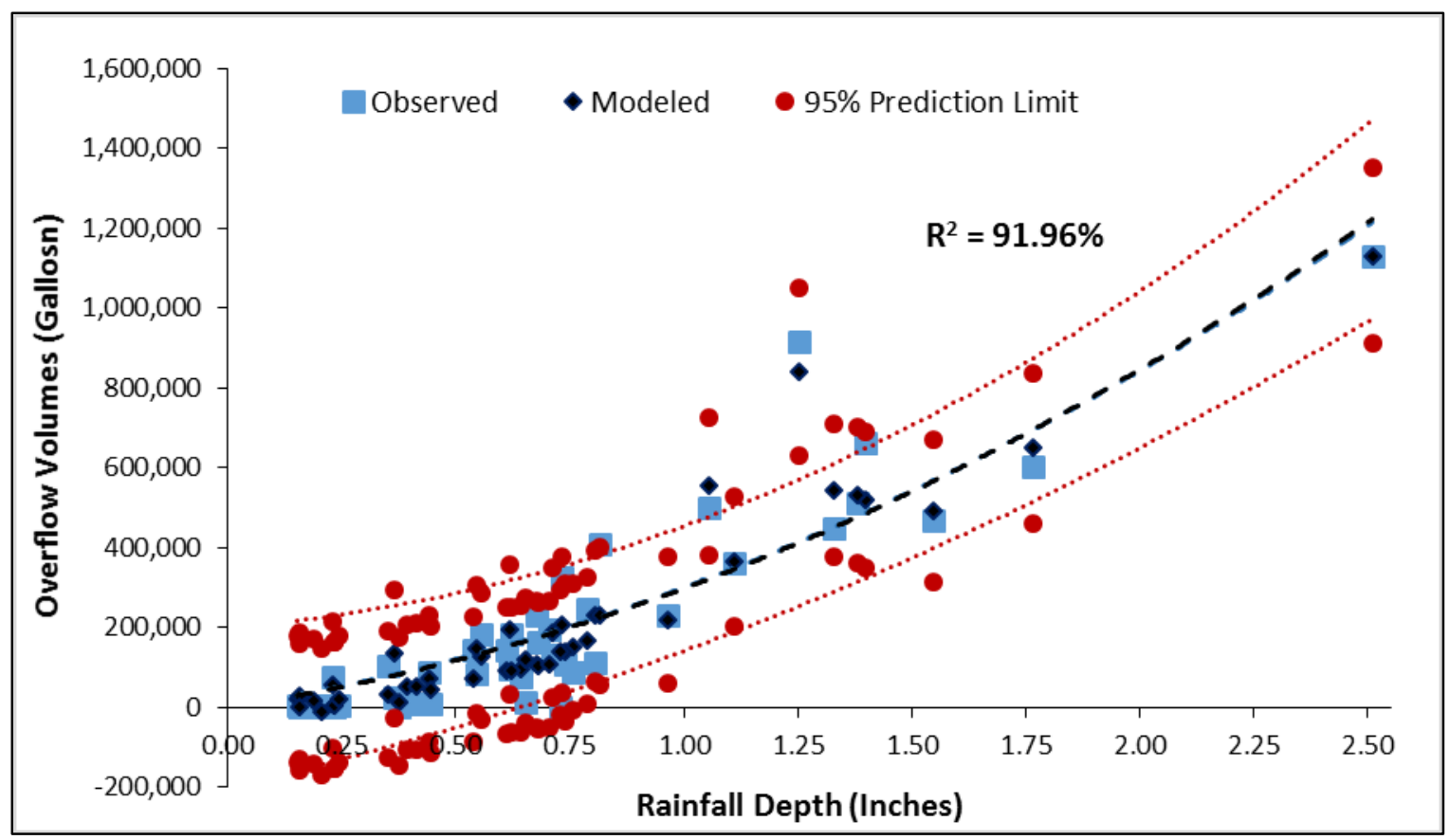

Figure 97 - Modeled and Observed Overflow Volumes versus the Rain Depth, CSO130

Since the intensity values and rainfall frequencies are related to each other, doing a sensitivity analysis on those parameters isn't applicable. The rainfall event and 3-day antecedent depth values are selected for testing the model's sensitivity to changes of these parameters and the results are showed in Figures 97 and 98. The intensity values and frequencies selected for both graphs are equal to average values of all events used in the calibration of the model. Both graphs represent a direct relationship between rainfall depth values and overflow volumes which is expected. 


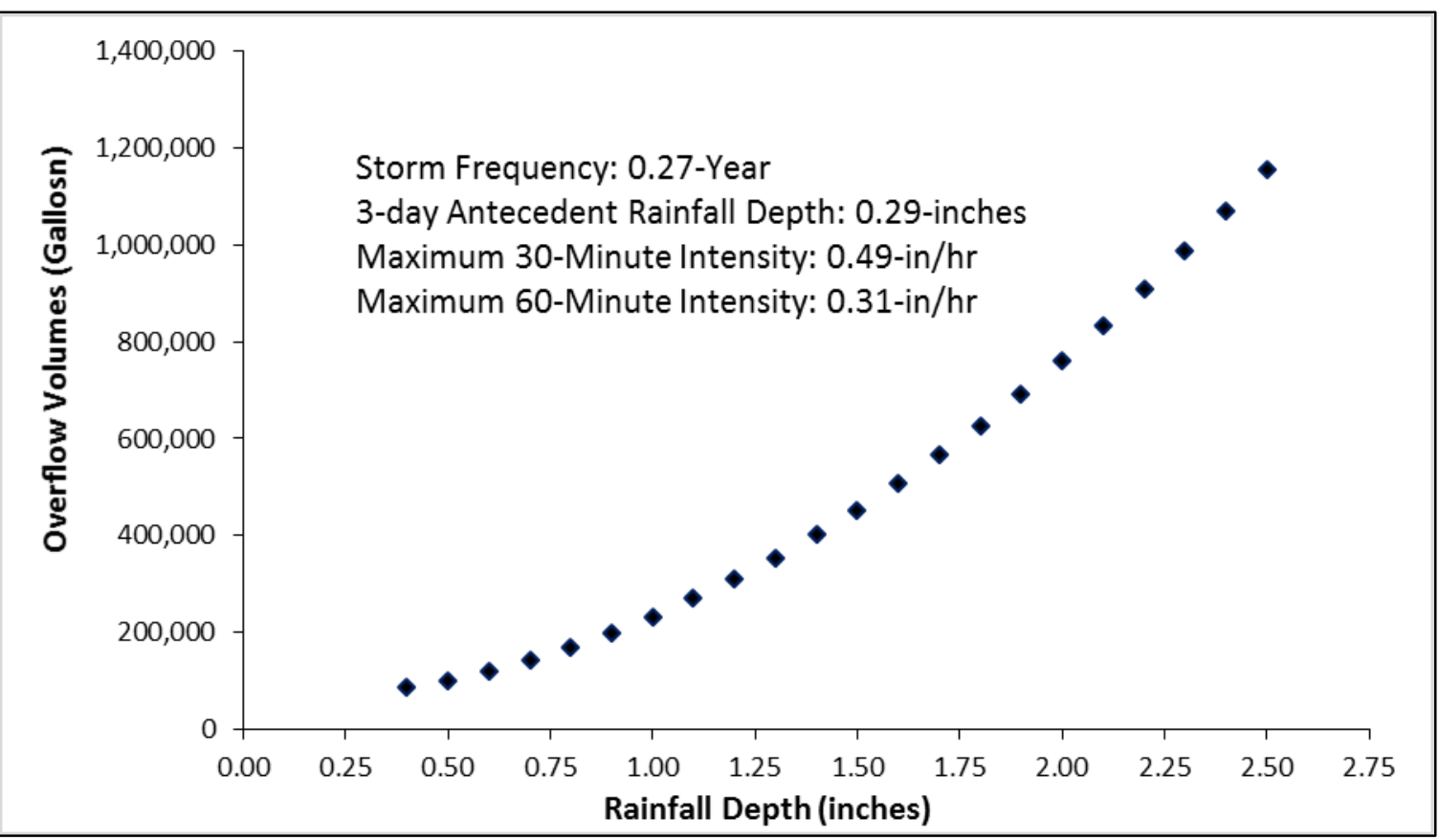

Figure 98 - Changes in Modeled Overflow Volume due to Changes of Rainfall Event Depth

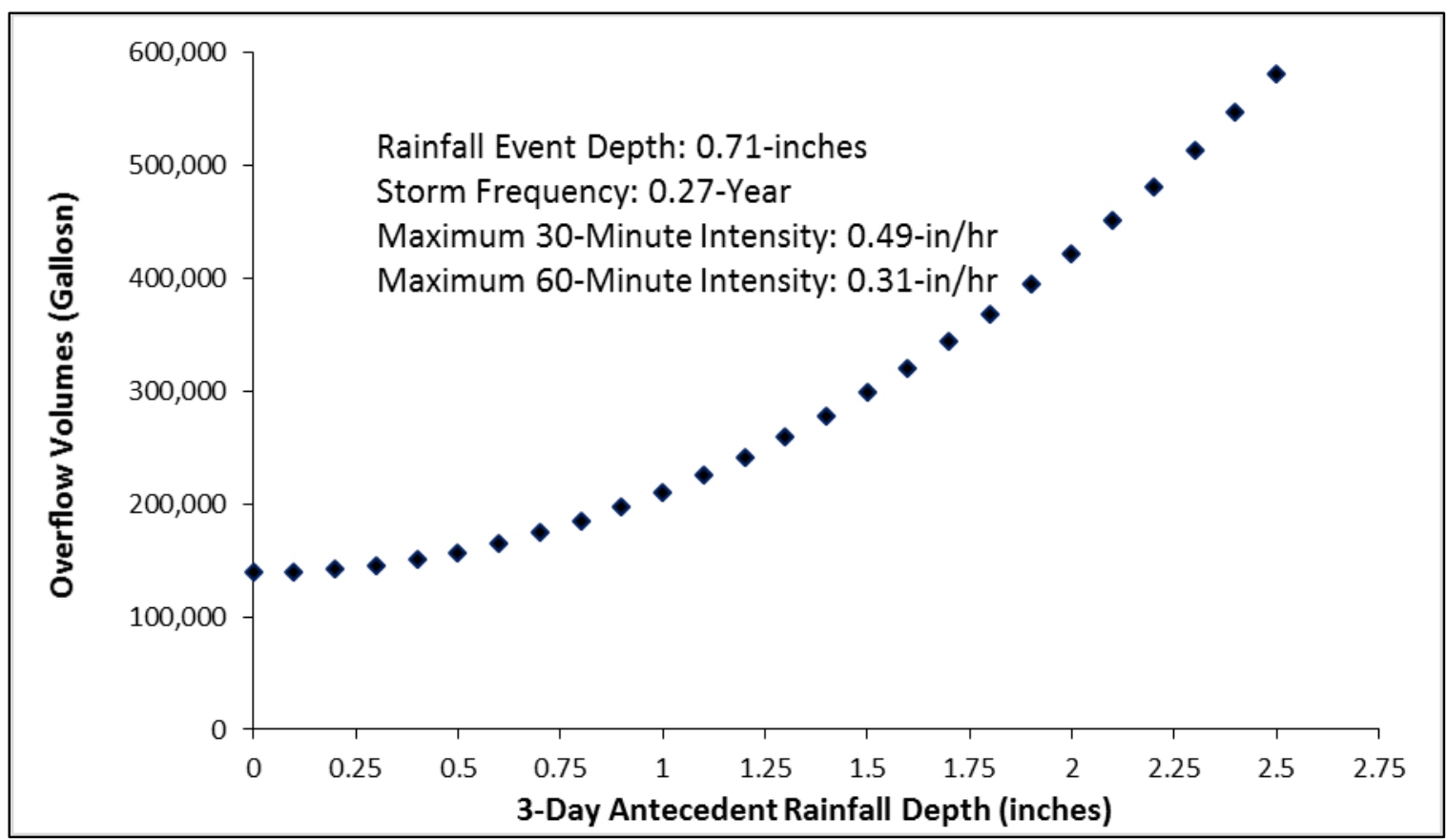

Figure 99 - Changes in Modeled Overflow Volume due to Changes of 3-Day Antecedent Rainfall Depth 


\subsubsection{Effectiveness of GI Controls}

Figure 100 shows the overflow volumes from CSO130 versus the rain depth values before (December 2011-August 2012) and after (May 2013-December 2013) construction of new GI Stormwater controls. The results show that after construction of the new GI controls, overflow volumes for rainfall events smaller than 0.7 inches have been significantly reduced except for one rainfall event with 0.43 inches depth which had a 2.6inch 3-day antecedent rainfall depth.

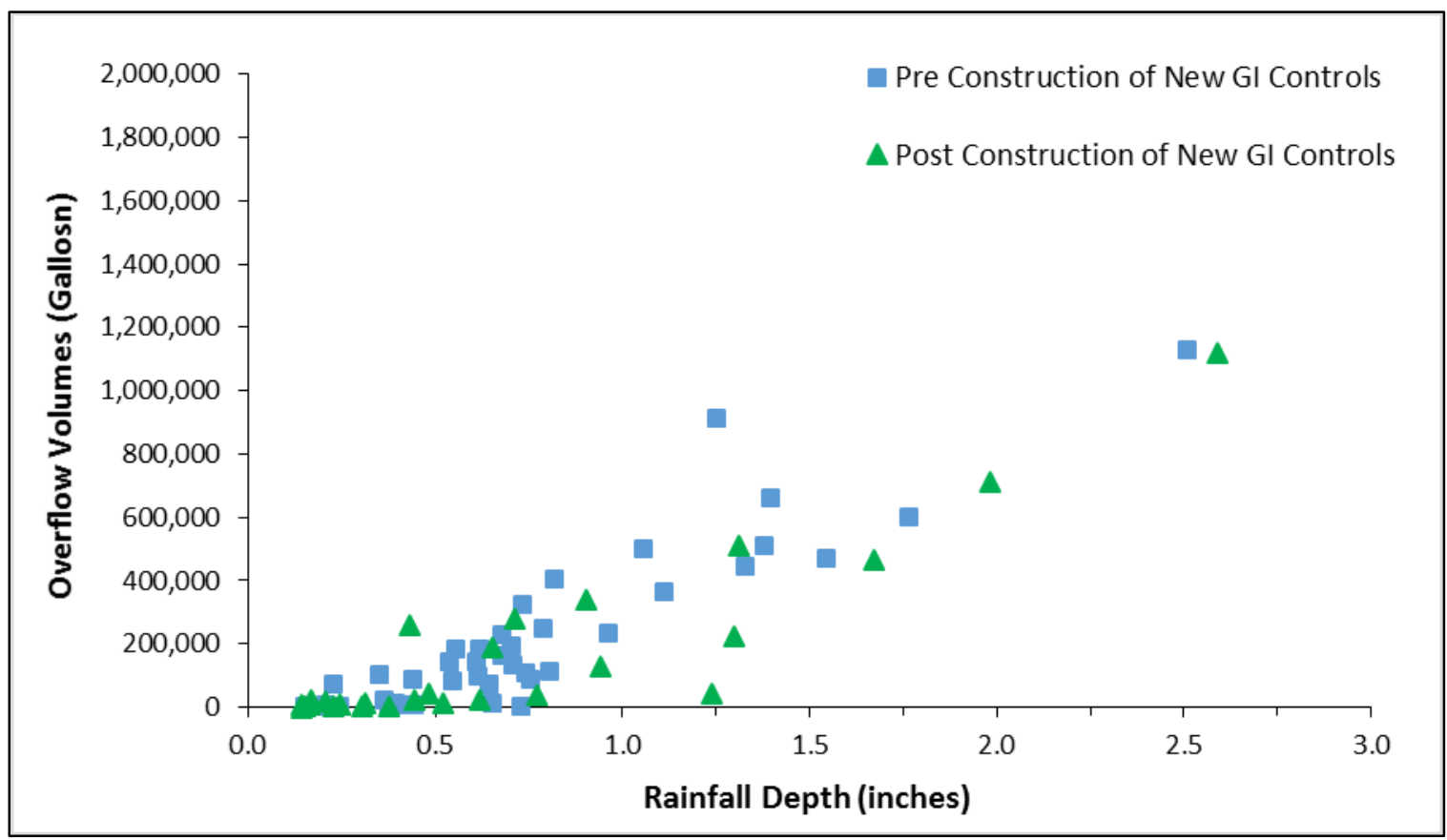

Figure 100 - Comparison of Overflow Volumes for Pre and Post Construction of New GI Controls

Eventually the developed regression model is used to predict the overflow volumes between May 2013 and December 2013. In this application the model is utilized as an assessment tool to compare the overflow volumes for pre and post development of new GI controls conditions as it was developed for the period of December 20111 and August 2012. It must be noted that since the model was only developed for rainfall events smaller 
than 2.5 inches, the events greater than this values are ignored for this effort. The observed overflow volumes and modeled are showed in Figure 101.

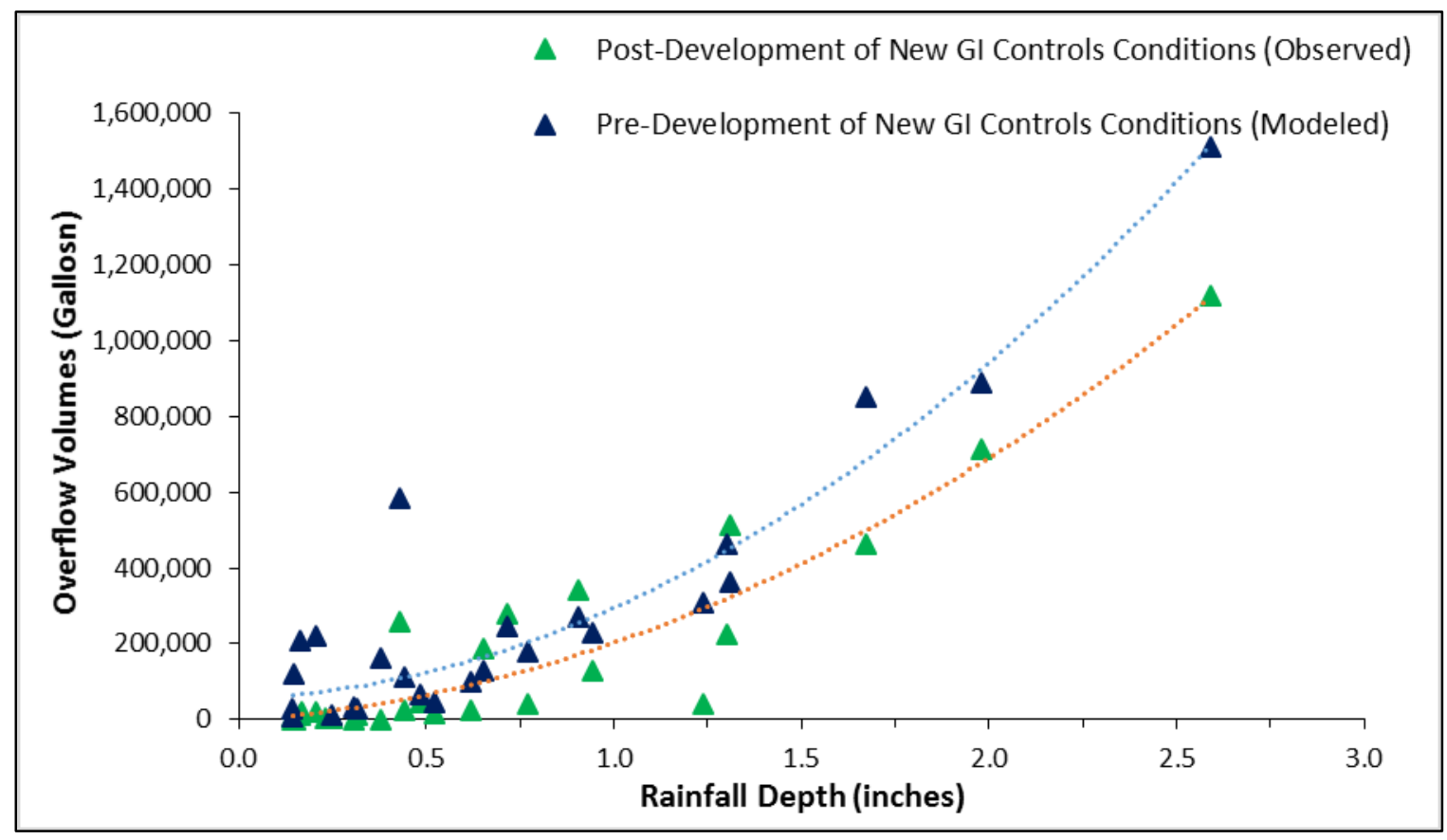

Figure 101 - Comparison of Observed and Modeled Overflow Volumes for Pre and Post Construction of New GI Controls Conditions

While Figures 100 and 101 indicate that after installation of new GI controls the overflow volumes have decreased to some extent, it seems that the level of reduction is not meeting MSD's initial objectives in CSO mitigation for CSO130 sewershed, which is to capture all storm events equal to or smaller than 1.09 inches. This is attributed to maintenance issues and the ongoing construction of GI controls during the monitoring period. Improvements in effectiveness of GI controls is expected after the completion of the construction and some additional needed rehabilitation work in the area, full implementation of the downspout disconnection program, and enactment of a regular 
maintenance plan. Conducting regular maintenance treatments is a key component for ensuring proper infiltration capacity of the GI controls.

The time period for this analysis was relatively short and it is suggested to conduct continuous monitoring of overflow volumes from CSO 130 in future and especially after conducting successful and regular maintenance treatments on the GI controls. 


\section{DISCUSSION OF THE RESULTS}

In this study the hydrological performance of two permeable pavement systems (19G and 19H) was monitored over the two-year course of this study. These two GI stormwater controls were installed in CSO130 sewershed in Louisville, Kentucky as part of a project which included installation of 16 other permeable pavement systems and 29 treeboxes. This project had the goal of mitigating the CSO problem in this sewershed by use of GI stormwater controls.

The hydrological performance of each GI control is designed for two primary functions: infiltration capacity and exfiltration performance. Unsatisfactory performance of any of these two functions could results in failure of GI stormwater controls. In this investigation both functions were monitored with a series of manual tests and electronically collected data. Chapters 4 through 6 explained the methodology and initial results from analysis of the collected data. This chapter discusses the analysis results and evaluates the individual hydrological performances of these two permeable pavement systems. 
The overall effectiveness of GI stormwater controls in CSO130 sewershed was evaluated as well. This was accomplished by comparing the pre and post construction overflow volumes from this basin. A regression analysis was also carried out to identify the factors affecting the overflow volumes from CSO130 sewershed for pre-construction conditions. The developed regression model was then used for post construction overflow events and the decrease in overflow volumes was determined. A discussion of the results from this analysis and the efficacies of installed green infrastructure controls are also provided in this chapter.

\subsection{Infiltration Capacity}

The infiltration capacity of a GI stormwater control is defined as its ability to capture the precipitation and surface storm runoff. The surface infiltration is mostly affected by surface clogging which forms when the sediments and debris in surface runoff get trapped between the gaps and pores on the surface of the GI control. Clogged sections of permeable pavements have a significant decreased infiltration capacity compared to unclogged sections.

Following the installation of GI controls $19 \mathrm{G}$ and $19 \mathrm{H}$ along the parking lanes of Adams Street, the field observations, manual field tests, and analysis of electronically collected data indicated the progression of clogging on the surface of both controls. The clogging advanced from the upgradient edge toward the downgradient edge and along the curbside. The concentration of clogging on the curbside was caused by the transverse slope of the street which also resulted in an active runoff flow width smaller than width of permeable pavement sections, see Figures 46 and 47. 
The progression of clogging on the surface of permeable pavement sections was monitored by TDR responses that were located along the curbside and at the depth (from pavement surface) of approximately 1.5 feet. The measurements from this array (curbside) of TDRs indicated a progression of clogging with a rate of 9-10 ft/in (feet per an inch of rainfall). The middle side TDRs showed that clogging at the center of permeable pavement section advanced with a similar pattern with curbside but at a slower rate $(\sim 5 \mathrm{ft} / \mathrm{in})$, see Figure 47.

The surface clogging rate is determined by identifying a threshold for TDR responses to separate the clogged and unclogged sections. The analysis of data based on first and last responses of curbside TDRs exceeding the selected threshold value are shown in Figure 44. The results suggested that for control 19G, the first TDR response exceeding $0.12 \mathrm{~cm}^{3} / \mathrm{cm}^{3}$ (Volumetric Water Content) indicate the infiltration of runoff flow at that location. The analysis also indicated that the $0.1 \mathrm{~cm}^{3} / \mathrm{cm}^{3}$ value is the threshold limit for separating the clogged and unclogged conditions on control 19G. Based on this threshold, a section of permeable pavement is declared clogged once the TDR at that location records a response greater than $0.1 \mathrm{~cm}^{3} / \mathrm{cm}^{3}$ for the last time.

Figure 47 not only shows that clogging at the center of permeable pavement section happens following the clogging at curbside but also that the distance increases as the curbside clogging advances toward the downgradient edge. This suggests that the clogging front is in form of a right triangle with one leg stretching as the clogging advances toward downgradient. 
To monitor the infiltration performance of permeable pavements, manual surface infiltration tests were also conducted periodically to evaluate the changes of infiltration rates over time. The initial tests following the installation of pavements showed that both controls had an average infiltration rate equal to $1250 \mathrm{in} / \mathrm{hr}$. The manual surface infiltration tests were repeated within one week before and after each maintenance treatment. The TDR data analysis and results from surface infiltration measurements are used to assess the effectiveness of each surface infiltration method.

While the manual surface infiltration results indicate short-term effectiveness of different maintenance treatments, the TDR data analysis and subsequently the calculated clogging rates, are utilized to monitor the long-term effectiveness of maintenance practices. During the course of study, control 19G was maintained five times using three different surface cleaning methods. The three methods included cleaning the pavement sections with a conventional street sweeper truck, by use of a pressurized airjet, and using an attachment prototype (Vac-Head) device. The different maintenance procedures were explained in detail in Chapter 6.

The analysis results showed that sweeper truck has been ineffective in restoring the short-term and long-term infiltration capacity of permeable pavement systems. This is suspected to be due to the fact that vacuum pressure of street sweeper has been unable to reach the full depth of articulated concrete blocks that are typically deeper than conventional interlocking concrete pavers.

Following sweeper truck, the articulated concrete blocks were cleaned by use of a pressurized airjet to blow out the trapped sediments from the gaps of concrete blocks. 
While the airjet method was successful in restoring the infiltration rates of the permeable pavements (short-term infiltration capacity), its long-term effectiveness decreased with each practice. Figure 102 compares the last measured TDR responses greater than 0.10 for the pressurized airjet maintenance treatments (control 19G) and the baseline performance. The graph shows that the slope of the each data set, which represents the clogging rate (feet per an inch of rainfall), significantly were increased by each airjet maintenance treatment. It must be noted that following the second airjet maintenance no measurements greater than 0.1 were collected by the TDRs located at 40 and 75 feet from the upgradient edge.

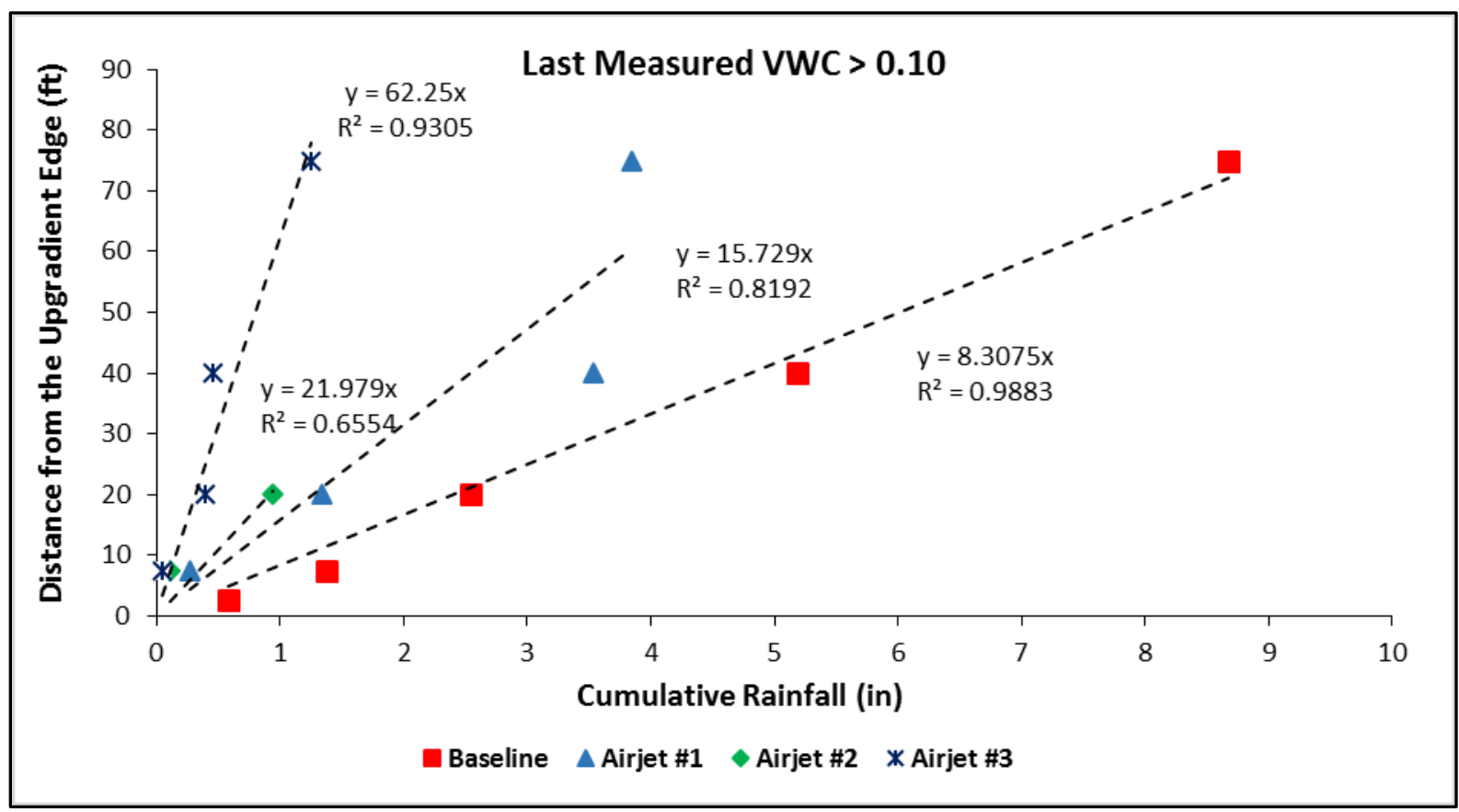

Figure 102 - Last Measured TDR Responses Greater than 0.1 Threshold for Airjet Maintenances, Control 19G

It is suspected that while each airjet maintenance removed the top layer of trapped sediments and debris, it was pushing some of it deeper inside the gap and under the curves of articulated concrete blocks. This restored the short-term infiltration rates but 
decreased the long-term infiltration capacity of permeable pavements. Since there was a concern associated with this method to blow the sediments further down and under the arches of concrete blocks, another maintenance treatment method was applied on control 19G.

The last tested maintenance treatment used an attachment connected to a conventional sewer cleaning truck, referred to as the Vac-Head maintenance treatment. The attachment used a rotating water jet to dislodge the trapped sediments which were vacuumed to the truck simultaneously. The TDR responses following this maintenance treatment indicated that clogging rate decreased to an even slower rate than baseline performance $(8.06 \mathrm{ft} /$ in versus baseline value of $8.95 \mathrm{ft} / \mathrm{in})$, see Figure 87 .

As previously discussed, the modeling effort showed that drainage area for control 19G had decreased by approximately $36 \%$ before conducting the Vac-Head maintenance treatment. By considering this fact and assuming constant drainage area for control 19G, the clogging rate after this maintenance would be possibly be equal to $12.59 \mathrm{ft} / \mathrm{in}$ :

$$
8.06 \mathrm{ft} / \mathrm{in} \div(1-0.36)=12.59 \mathrm{ft} / \mathrm{in}
$$

The new clogging rate is smaller than values from all previous maintenance treatments. This is satisfactory especially considering the fact that three airjet maintenance treatments had possibly caused permanent damage to the infiltration performance of permeable pavements by pushing the sediments and clogging materials into the arches of concrete blocks. The manual surface infiltration rates also confirmed that the Vac-Head maintenance, unlike the airjet maintenances, restored the short-term infiltration capacity at the most upgradient test location ( $2.5 \mathrm{ft}$ from upgradient edge). This location was heavier 
clogged than other test locations and its infiltration rates had never been restored to its baseline value with any of the previous maintenance treatments.

\subsection{Exfiltration Performance}

The exfiltration performance of a GI control is defined as its ability to pass the captured runoff volume to the underlying and surrounding natural soil layers. This performance is affected by hydraulic conductivity, and subsequently exfiltration rates, of natural soil layers. In this study, measuring horizontal infiltration rates of soil layers were not feasible but a falling-head test was used during construction to estimate the vertical infiltration rates of underlying soil layers at the bottom of trenches. The results from the test showed that control $19 \mathrm{H}$ had a more permeable underlying soil compared to $19 \mathrm{G}$, see Table 4.

As previously discussed in Chapter 4, the pressure transducers data were utilized to estimate the recession and exfiltration rates for controls $19 \mathrm{G}$ and $19 \mathrm{H}$. The analyzed data showed that these rates were greater for higher levels of water which is expected due to the direct relationship of hydraulic head and exfiltration performance. Figures 34 through 36 confirmed that for low levels of water inside the trench, control $19 \mathrm{H}$ showed higher recession and exfiltration rates compared to control 19G, which was consistent with results from falling head test conducted during construction. These figures indicate that despite the low permeability of underlying soil layers for control 19G compared to control $19 \mathrm{H}$, 19G had surrounding soil layers with higher permeability at its top levels.

Similar to the infiltration performance, the most critical factor adversely affecting the exfiltration performance is clogging. Clogging is suspected to form at the bottoms of 
controls 19G and 19H and at the interface of \#3 storage layer and underlying soil layer. The sources of clogging material at the bottom of trench include: the remaining debris from construction of GI controls, attached solids from \#3 and \#57 stone layers, loose fine soil materials coming off from sidewalls of trench and storage gallery, and debris and suspended solids carried by surface runoff. The analysis of pressure transducers data shows significant decrease of exfiltration rates following the first few rain events which is suspected to be caused by the first three sources mentioned. This decrease was greater for control 19G compared to control 19H, see Figures 48 and 49. After these initial events, the captured runoff volume is believed to be the major source contributing to clogging at the bottom of the trenches.

The modeling results showed that generally $95 \%$ of exfiltration from controls $19 \mathrm{G}$ and $19 \mathrm{H}$ takes place through the lateral side walls of each control. The exfiltration from bottom of the trenches becomes more significant for low levels of water. This indicates that clogging inside the trenches would probably wouldn't affect the exfiltration performance of controls $19 \mathrm{G}$ and $19 \mathrm{H}$ greatly for higher level of water.

Another factor contributing to changes of exfiltration rates is the temperature variation at the bottom of the GI controls. The influence of the seasonal temperature changes on exfiltration rates throughout the course of study are evident in Figures 48 and 49. Changes in temperature affect the dynamic viscosity of water that subsequently affects the hydraulic conductivity values (and exfiltration rates) of soil layers. The estimated exfiltration rates showed their maximum and minimum annual values for late summer (August, September) and late winter (February, March), respectively. 
Further analysis based on estimated exfiltration rates and temperature values showed that temperature decency of exfiltration performance for control $19 \mathrm{G}$ during the years 2012 and 2013, and for control 19H during year 2012, is mostly due to changes of water viscosity. On the other hand the results suggest that for control 19H during 2013 a combination of changes of water viscosity and another factor was causing the temperature dependency and seasonal variations of exfiltration rates.

It is hypothesized that exfiltration performance of control 19H during 2013 is affected by its infiltration performance. In this year the surface of control $19 \mathrm{H}$ was mostly clogged and didn't experience any successful maintenance treatments to restore its infiltration capacity. As a result, during 2013 control 19H captured approximately 50\% runoff volume compared to previous year. The smaller captured runoff volume disturbed the balance, established during the 2012, between the entering organic sediments and their decomposition rate for 2013. The decomposition rate of organic material is typically increased for higher temperatures and during summer time. The clogging material at surface of control $19 \mathrm{H}$ has also probably performed as a filtering layer by reducing the amount of sediments entering the GI control. Combination of these two increased the control 19H's exfiltration rates of the GI control during 2013 compared to previous year and especially for summer time.

\subsection{Effectiveness of GI Controls in CSO Mitigation}

As previously mentioned, Louisville MSD's plan was to achieve a level of CSO mitigation for CSO130 basin by using GI stormwater controls. The proposed GI stormwater controls to achieve this goal included a total number of 18 permeable pavement 
strips and 29 treeboxes. The majority of these GI controls were installed during first half of year 2013.

An analysis was carried out to evaluate the total effectiveness of these GI stormwater controls in achieving the CSO mitigation. The goal of the project was to eliminate CSOs for events smaller than 1.09 inches of rainfall, which corresponds to the $9^{\text {th }}$ largest rainfall event for the design rainfall year (2001). The pre and post construction overflow volumes from CSO130 was compared and the results showed that following the construction of GI stormwater controls, rainfall events up to 0.70 inch has been controlled.

To better assess the improvements in CSO mitigation after the construction of new GI controls in CSO130 sewershed, a regression analysis was utilized. In this analysis a regression equation was developed to model the overflow volumes from CSO130 based on pre-construction conditions. This model was then used for post construction rain events to predict the overflow volumes by assuming no GI controls were installed in this sewershed.

The results from this analysis showed that following the construction of new GI controls the overflow volumes were decreased, see Figure 100. The overflow volumes saved by installing the new GI controls in second half of year 2013 are approximately equal to $2.6 \mathrm{MG}$, see Table 36 . It must be noted that since the regression equation in this analysis was developed and calibrated for rain events smaller than 2.5 inches, three rain events exceeding this threshold are excluded from calculation provided in Table 36. Actual overflows and volumes saved by installing GI controls including these three events are greater than values provided in Table 36. 
Table 36 - Predicted and Observed Overflow Volumes for Pre and Post Construction Conditions

Total Observed Overflow Total Overflow Volume Predicted Overflow Volume Saved

Volume (Post Construction) (Assuming No Construction)

$4.5 \mathrm{MG} 7.1 \mathrm{MG} \quad 2.6 \mathrm{MG}$

It must be mentioned that during the course of this analysis, the construction of few GI controls were not complete and the permeable pavement systems, except for controls 19G and 19H, had never been maintained. It is expected to see improvements in effectiveness of GI stormwater controls once the construction is completed and a regular maintenance plan is applied. 


\section{CONCLUSIONS AND FUTURE WORK}

\subsection{Conclusions}

The objective of this research was to quantitatively assess the hydrological performance of two green infrastructure storm water control systems. Unlike many previous research studies, this work had the opportunity to monitor the changes in hydrological performance in full scale and real urban environment over a two year study period. To complete the monitoring effort a comprehensive monitoring plan was devised during the design and, during construction the GI systems were heavily embedded with electronic sensors. Remote data collection from these sensors, along with frequent field visits and measurements, provided ample data to evaluate changes in infiltration and exfiltration performances of both GI controls.

As mentioned in Chapter 1 of this document, the two systems had larger drainage ratios compared to traditional applications of permeable pavements. The large drainage areas combined with heavy debris conditions of the urban environment, resulted in accelerated surface clogging and changes of infiltration performance of both permeable pavements. This was favorable for research purposes as infiltration performance observed in the two-year study period provided greater data than a normal application of the GI controls would have. 
A spreadsheet model was subsequently developed using the available data to monitor the changes in infiltration performance and assess the effectiveness of maintenance procedures in restoring infiltration capacity. The results indicated that clogging initially progressed from upgradient to downgradient and along the curbside with a rate equal to approximately 10 feet per an inch of rainfall.

The developed model also allowed the calculation of captured stormwater runoff while considering the intra-event exfiltration. The output of the model and calculated captured volumes indicated that clogged permeable pavement sections still maintain a percentage of their infiltration capacity. The modeling results provided a better understanding of the exfiltration processes as the GI systems transferred the stormwater runoff volume to the natural aquifers. Seasonal changes in system performance were observed and attributed to changes in dynamic viscosity of water caused by variation of temperature. The model also showed that a significant percentage of exfiltration is occurring horizontally, through the walls of deep trenches excavated underneath the permeable pavements, and not the through the bottom area. This is especially important as it will promulgate a design modification for GI stormwater controls on soils with low hydraulic conductivity values.

The modeling results also provided the opportunity to assess the effectiveness of various maintenance operations performed on the permeable pavement systems. By analyzing the TDR data, the rate and extent of surface clogging could be closely monitored. While many maintenance operations may provide a brief short-term improvement in infiltration performance, a deep cleaning is necessary to reestablish an effective long-term infiltration. The critical evaluation of maintenance operations in collaboration with the 
manufacturer resulted in improving the maintenance protocols and equipment for articulated concrete blocks. The results indicated that permeable pavements with large impermeable drainage areas, even in high debris environments, could provide sufficient and favorable infiltration performance with frequent application of a suitable maintenance method.

Ultimately, this study worked to assess the effectiveness of multiple GI controls for reducing storm water overflows within a specific watershed. Multiple tree boxes and permeable pavement systems were installed in the CSO130 sewershed and the effectiveness of the suite of GI systems was assessed by comparing pre- and postconstruction overflow volumes. Because of the dynamic nature of rainfall and subsequently runoff a regression analysis was performed to first identify the factors significantly affecting the overflow volumes and second to develop a model to compare the pre and post-construction conditions. The results showed that almost 2.6 million gallons of overflows were eliminated from the combined sewer system following the construction of all GI stormwater controls in CSO130 sewershed and during the second half of year 2013. The regression analysis effort also identified other factors playing a role in overflow volumes such as 3-day antecedent rainfall depth and rainfall intensity. And finally, the model determined that the storm duration did not significantly affect the overflows volumes. The lack of correlation to storm duration could be of importance when modeling the runoff flow from other areas with combined sewer systems.

As a final conclusion, based on the results of this study the following is suggested to be considered in future design and applications of the permeable pavement systems: 
- In similar applications of permeable pavement systems as long narrow strips, it is suggested to decrease the width of the pavement section to fully exploit the infiltration performance of the permeable pavements. This would allow a decrease in construction cost or application of a longer strip which could decrease the impermeable area draining to the permeable pavement, resulting in better infiltration performance of the system.

- Application of a pre-treatment setup upgradient of the permeable pavement system is suggested to capture the debris and organic material in stormwater runoff. This would potentially prolong the surface clogging progression and result in decrease in maintenance associated costs.

\subsection{Future Work}

The objectives of this research work were narrowly focused to assess the individual performance of the green infrastructure stormwater control systems constructed within CSO130 sewershed. While some interesting and design changing information was learned, there are still many opportunities to improve green infrastructure stormwater control systems specifically related to large scale implementation, water quality and design improvement.

Large scale implementation - Since this study primarily focused on individual hydrological performance of permeable pavements it is suggested for the future research on the effectiveness of a suite of GI stormwater control measures from a larger scale, similar to effort conducted in Chapter 7 of this document. Such macro-scale monitoring and associated techniques are critical especially as more communities move toward applications of GI stormwater controls to address the CSO issues. 
Water quality - Current stormwater regulations are focused primarily on reducing the overall flow volume, a quantity measure. It is evident that forthcoming stormwater regulations will focus on both stormwater quantity and stormwater quality. The results from stormwater quality study combined with hydrological performance results presented in this document, could provide a more robust assessment of permeable pavement performance and serve as a decision factor for future application of GI stormwater control measures.

Design improvements - Prior to this study, little was known about the infiltration and exfiltration characteristics of installed stormwater control systems. By including the instrumentation in these systems, a much better understanding of operational performance could be obtained. Through detailed data analysis, it was observed that surface infiltration performance, which is greatly affected by surface clogging, is a key component in hydrological performance of permeable pavements. This is especially of importance in urban environments with increased amount of debris and organic materials. The specific design details of each stormwater control system should be adapted to account for the specific onsite characteristics. There is a need for a rational design guide that would account for in situ permeability, drainage ratio, anticipated debris loading and other key factors likely encountered in real world applications. 


\section{REFERENCES}

\section{Abbott, C. L., and Comino-Mateos, L. (2003). "IN-SITU HYDRAULIC PERFORMANCE OF A PERMEABLE PAVEMENT SUSTAINABLE URBAN DRAINAGE SYSTEM." Water and Environment Journal, 17(3), 187-190.}

Al-Rubaei, A., Stenglein, A., Viklander, M., and Blecken, G. (2013). "Long-Term Hydraulic Performance of Porous Asphalt Pavements in Northern Sweden." Journal of Irrigation and Drainage Engineering, 139(6), 499-505.

Arnold, C. L., and Gibbons, C. J. (1996). "Impervious Surface Coverage: The Emergence of a Key Environmental Indicator." Journal of the American Planning Association, 62(2), 243-258.

ASTM (2009). "Standard Test Method for Infiltration Rate of In-place Pervious Concrete "West Conshohocken, PA.

ASTM (2009). "Standard test method for infiltration rate of soils in field using doublering infiltrometer." ASTM D3385-09, American Society for Testing Materials (ASTM) International, West Conshohocken, PA.

Badwaterjournal.com (2010). "MSD 'GREEN INFRASTRUCTURE’ PROJECTS MOVE FORWARD."

Baladès, J. D., Legret, M., and Madiec, H. (1995). "Permeable pavements: Pollution management tools." Water Science and Technology, 32(1), 49-56.

Bean, E., Hunt, W., and Bidelspach, D. (2007a). "Field Survey of Permeable Pavement Surface Infiltration Rates." Journal of Irrigation and Drainage Engineering, 133(3), 249-255.

Bean, E., Hunt, W., and Bidelspach, D. (2007b). "Evaluation of Four Permeable Pavement Sites in Eastern North Carolina for Runoff Reduction and Water 
Quality Impacts." Journal of Irrigation and Drainage Engineering, 133(6), 583592.

Booth, D. B., Hartley, D., and Jackson, R. (2002). "FOREST COVER, IMPERVIOUSSURFACE AREA, AND THE MITIGATION OF STORMWATER IMPACTS1." JAWRA Journal of the American Water Resources Association, 38(3), 835-845.

Booth, D. B., and Leavitt, J. (1999). "Field Evaluation of Permeable Pavement Systems for Improved Stormwater Management." Journal of the American Planning Association, 65(3), 314-325.

Borst, M., Rowe, A., Stander, E. K., and O'Connor, T. P. (2010). "Surface Infiltration Rates of Permeable Surfaces: Six Month Update (November 2009 through April 2010)." EPA/600/R-10/083, US Environmental Protection Agency Office of Research and Development,, Washington, D.C.

Braga, A., Horst, M., and Traver, R. (2007). "Temperature Effects on the Infiltration Rate through an Infiltration Basin BMP." Journal of Irrigation and Drainage Engineering, 133(6), 593-601.

Brattebo, B. O., and Booth, D. B. (2003). "Long-term stormwater quantity and quality performance of permeable pavement systems." Water Research, 37(18), 43694376.

Brown, R., and Borst, M. (2013). "Assessment of Clogging Dynamics in Permeable Pavement Systems with Time Domain Reflectometers." Journal of Environmental Engineering, 139(10), 1255-1265.

Brown, R., Borst, M., and O'Connor, T. (2012). "Analysis of Instrumentation Selection and Placement to Monitor the Hydrologic Performance of Permeable Pavement Systems and Bioinfiltration Areas at the Edison Environmental Center in New Jersey." World Environmental and Water Resources Congress 2012, 223-234.

Campbell Scientific, I. (2012). CS650 and CS655 Water Content Reflectometers: Instruction Manual, Campbell Scientific, Inc, Logan, Utah.

Chai, L., Kayhanian, M., Givens, B., Harvey, J., and Jones, D. (2012). "Hydraulic Performance of Fully Permeable Highway Shoulder for Storm Water Runoff Management." Journal of Environmental Engineering, 138(7), 711-722. 
Chopra, M., Kakuturu, S., Ballock, C., Spence, J., and Wanielista, M. (2010). "Effect of Rejuvenation Methods on the Infiltration Rates of Pervious Concrete Pavements." Journal of Hydrologic Engineering, 15(6), 426-433.

Collins, K., Hunt, W., and Hathaway, J. (2008). "Hydrologic Comparison of Four Types of Permeable Pavement and Standard Asphalt in Eastern North Carolina." Journal of Hydrologic Engineering, 13(12), 1146-1157.

Coughlin, J., Campbell, C., and Mays, D. (2012). "Infiltration and Clogging by Sand and Clay in a Pervious Concrete Pavement System." Journal of Hydrologic Engineering, 17(1), 68-73.

CWP (2003). Impacts of Impervious Cover on Aquatic Systems, Center for Watershed Protection, Ellicott City, MD.

Decagon-Devices (2011). "Minidisk infiltrometer: User's manual version 9.", Decagon Devices Inc., Pullman, WA.

Deo, O., Sumanasooriya, M., and Neithalath, N. (2010). "Permeability Reduction in Pervious Concretes due to Clogging: Experiments and Modeling." Journal of Materials in Civil Engineering, 22(7), 741-751.

District Department of the Environment Watershed Protection Division (2013). "Draft District of Columbia Stormwater Management Guidebook "District of Columbia

Dreelin, E. A., Fowler, L., and Ronald Carroll, C. (2006). "A test of porous pavement effectiveness on clay soils during natural storm events." Water Research, 40(4), 799-805.

El Samrani, A. G., Lartiges, B. S., Ghanbaja, J., Yvon, J., and Kohler, A. (2004). "Trace element carriers in combined sewer during dry and wet weather: an electron microscope investigation." Water Research, 38(8), 2063-2076.

Emerson, C., and Traver, R. (2008). "Multiyear and Seasonal Variation of Infiltration from Storm-Water Best Management Practices." Journal of Irrigation and Drainage Engineering, 134(5), 598-605.

Emerson, C. H. (2008). "Evaluation of Infiltration Practices as a Means to Control Stormwater Runoff." Doctor of Philosophy, Villanova University, Villanova, PA. 
Emerson, C. H., Wadzuk, B. M., and Traver, R. G. (2010). "Hydraulic evolution and total suspended solids capture of an infiltration trench." Hydrological Processes, 24(8), 1008-1014.

Even, S., Mouchel, J.-M., Servais, P., Flipo, N., Poulin, M., Blanc, S., Chabanel, M., and Paffoni, C. (2007). "Modelling the impacts of Combined Sewer Overflows on the river Seine water quality." Science of The Total Environment, 375(1-3), 140-151.

Even, S., Poulin, M., Mouchel, J.-M., Seidl, M., and Servais, P. (2004). "Modelling oxygen deficits in the Seine River downstream of combined sewer overflows." Ecological Modelling, 173(2-3), 177-196.

Fassman, E., and Blackbourn, S. (2010). "Urban Runoff Mitigation by a Permeable Pavement System over Impermeable Soils." Journal of Hydrologic Engineering, 15(6), 475-485.

Fassman, E., and Blackbourn, S. (2011). "Road Runoff Water-Quality Mitigation by Permeable Modular Concrete Pavers." Journal of Irrigation and Drainage Engineering, 137(11), 720-729.

Ferguson, B. K. (2005). Porous Pavements, Taylor \& Francis, Boca Raton, FL.

Field, R., Sullivan, D., and Tafuri, A. N. (2003). Management of Combined Sewer Overflows, Taylor \& Francis, Boca Raton, FL.

Galster, J. C. P., Frank J.; Hargreaves, Bruce R.; Morris, Donald P.; Peters, Stephen C.; Weisman, Richard N. (2006). "Effects of Urbanization on Watershed Hydrology: The Scaling of Discharge with Drainage Area." Geology, 34(9), 713-716.

Gilbert, J. K., and Clausen, J. C. (2006). "Stormwater runoff quality and quantity from asphalt, paver, and crushed stone driveways in Connecticut." Water Research, 40(4), 826-832.

Haselbach, L. M., Valavala, S., and Montes, F. (2006). "Permeability predictions for sand-clogged Portland cement pervious concrete pavement systems." Journal of Environmental Management, 81(1), 42-49. 
Haster, T., and James, W. (1994). "Predicting Sediment Yield in Storm-Water Runoff from Urban Areas." Journal of Water Resources Planning and Management, 120(5), 630-650.

Hillel, D. (1998). Environmental Soil Physics, Academic Press.

Hou, L., Feng, S., Ding, Y., Zhang, S., and Hou, Z. (2008). "Experimental study on rainfall-runoff relation for porous pavements." Hydrology Research, 39(3).

Jones, M. P., and Hunt, W. F. (2010). "Performance of rainwater harvesting systems in the southeastern United States." Resources, Conservation and Recycling, 54(10), 623-629.

Kayhanian, M., Anderson, D., Harvey, J. T., Jones, D., and Muhunthan, B. (2012). "Permeability measurement and scan imaging to assess clogging of pervious concrete pavements in parking lots." Journal of Environmental Management, 95(1), 114-123.

LeBoon, M., and Traver, R. (2007). "An Infiltration Model of an Underground Rock Storage Bed Infiltration BMP." World Environmental and Water Resources Congress 2007, 1-10.

Li, H., Harvey, J. T., Holland, T. J., and Kayhanian, M. (2013). "The use of reflective and permeable pavements as a potential practice for heat island mitigation and stormwater management." Environmental Research Letters, 8(1).

Lin, C., Greenwald, D., and Banin, A. (2003). "Temperature dependence of infiltration rate during large scale water recharge into soils." Soil Sci. Soc. Am. J., 67(2), 487493.

Moghadasi, J., Müller-Steinhagen, H., Jamialahmadi, M., and Sharif, A. (2004). "Theoretical and experimental study of particle movement and deposition in porous media during water injection." Journal of Petroleum Science and Engineering, 43(3-4), 163-181.

Montgomery, D. C., Peck, E. A., and Vining, G. G. (2001). Introduction to Linear Regression Analysis, Wiley, New York. 
MSD (2009). "Chapter 4: Selection of a Final CSO Long-Term Control Plan." Integrated Overflow Abatement Plan: Final CSO Long-Term Control Plan Louisville and Jefferson County Metropolitan Sewer District (MSD), Louisville, KY, 1-44.

MSD (2011). Green Infrastructure Design Manual: Green Management Practices and Design Strategies to Manage Stormwater in our Community, Louisville and Jefferson County Metropolitan Sewer District, Louisville, KY.

MSD (2013a). "Federal Consent Decree." <http://msdprojectwin.org/About-Us/FederalConsent-Decree.aspx>.

MSD (2013b). "Integrated Overflow Abatement Plan (IOAP)." $<$ http://msdprojectwin.org/About-Us/Integrated-Overflow-Abatement-PlanIOAP.aspx $>$.

NCHRP (2006). "Evaluation of Best Management Practices for Highway Runoff Control." NCHRP REPORT 565, Transportation Research Board.

Nowak, D. J., Crane, D. E., and Stevens, J. C. (2006). "Air pollution removal by urban trees and shrubs in the United States." Urban Forestry \& Urban Greening, 4(34), 115-123.

NRDC (2011). "Rooftops to Rivers II: Green Strategies for Controlling Stormwater and Combined Sewer Overflows." Natural Resources Defence Council, New York, NY.

PaveDrain (2011). "Sustainable Stormwater Solutions." $\langle$ http://www.pavedrain.com/pdf/PaveDrain-Brochure.pdf $\rangle$.

Pazwash, H. (2011). Urban Storm Water Management, Taylor \& Francis, Boca Raton, FL.

Pitt, R., Chen, S., Clark, S., Swenson, J., and Ong, C. (2008). "Compaction's Impacts on Urban Storm-Water Infiltration." Journal of Irrigation and Drainage Engineering, 134(5), 652-658.

Pitt, R., Lantrip, J., Harrison, R., Henry, C. L., Xue, D., and O'Connor, T. P. (1999). "Infiltration through disturbed urban soils and compost-amended soil effects on 
runoff quality and quantity." Rep. No. EPA/600/R-00/16, U.S. Environmental Protection AgencyCincinnati, OH.

Pyke, C., Warren, M. P., Johnson, T., LaGro Jr, J., Scharfenberg, J., Groth, P., Freed, R., Schroeer, W., and Main, E. (2011). "Assessment of low impact development for managing stormwater with changing precipitation due to climate change." Landscape and Urban Planning, 103(2), 166-173.

Sansalone, J., Kuang, X., Ying, G., and Ranieri, V. (2012). "Filtration and clogging of permeable pavement loaded by urban drainage." Water Research, 46(20), 67636774.

Scholz, M., and Grabowiecki, P. (2007). "Review of permeable pavement systems." Building and Environment, 42(11), 3830-3836.

Schueler, T. R. (1994). "The Importance of Imperviousness." Watershed Protection Techniques, 1(3), 100-111.

SEMCOG (2008). "Low Impact Development Manual for Michigan: A Design Guide for Implementors and Reviewers."Detroit, MI.

Shuster, W. D., Bonta, J., Thurston, H., Warnemuende, E., and Smith, D. R. (2005). "Impacts of impervious surface on watershed hydrology: A review." Urban Water Journal, 2(4), 263-275.

U.S.EPA (1999a). "Combined Sewer Overflow: O\&M Fact Sheet " EPA 832-F-99-039, U.S.EPA.

U.S.EPA (1999b). "Storm Water Technology Fact Sheet: Vegetated Swales." 832-F-99006, U.S.EPA.

U.S.EPA (2000). "Low Impact Development (LID): A Literature Review." EPA-841-B00-005 U.S.EPA.

U.S.EPA (2002). "Combined Sewer Overflows: CSO Control Policy ".

U.S.EPA (2003). "Fact Sheet : Clean Water Is Everybody's Business." EPA 841-F-03003, U.S.EPA. 
U.S.EPA (2004). "Report to Congress: Impacts and Control of CSOs and SSOs." U.S. Environmental Protection Agency, Washington, D.C.

U.S.EPA (2009a). "Porous Asphalt Pavement ", $<$ http://cfpub.epa.gov/npdes/stormwater/menuofbmps/index.cfm?action=browse\& Rbutton $=$ detail $\& b m p=135 \&$ minmeasure $=5>$.

U.S.EPA (2009b). "Pervious Concrete Pavement ", $<$ http://cfpub.epa.gov/npdes/stormwater/menuofbmps/index.cfm?action=browse\& Rbutton $=$ detail $\& b m p=137 \&$ minmeasure $=5>$.

U.S.EPA (2009c). "Permeable Interlocking Concrete Pavement." $<$ http://cfpub.epa.gov/npdes/stormwater/menuofbmps/index.cfm?action=browse\& Rbutton $=$ detail $\& b m p=136 \&$ minmeasure $=5>$.

U.S.EPA (2012a). "National Enforcement Initiatives for Fiscal Years 2008 - 2010:Clean Water Act: Municipal Sewer Overflows (Combined Sewer Overflows \& Sanitary Sewer Overflows)."

U.S.EPA (2012b). "Combined Sewer Overflows." <http://cfpub.epa.gov/npdes/home.cfm?program_id=5>.

U.S.EPA (2012c). "Stormwater Management Best Practices." $<$ http://www.epa.gov/oaintrnt/stormwater/best practices.htm>.

U.S.EPA (2012d). "Green Landscaping: Greenacres." $<$ http://www.epa.gov/greenacres/landuse.html〉.

U.S.EPA (2013a). "Clean Water Act (CWA)." $\langle$ http://www.epa.gov/agriculture/lcwa.html〉.

U.S.EPA (2013b). "Green Infrastructure." $<$ http://water.epa.gov/infrastructure/greeninfrastructure/index.cfm〉.

U.S.EPA (2013c). "Rainwater Harvesting: Conservation, Credit, Codes, and Cost: Literature Review and Case Studies." EPA-841-R-13-002, U.S.EPA. 
Valavala, S., Montes, F., and Haselbach, L. (2006). "Area-Rated Rational Coefficients for Portland Cement Pervious Concrete Pavement." Journal of Hydrologic Engineering, 11(3), 257-260.

Van Heystraeten, G., and Moraux, C. (1990). "Ten Years' Expreince of Porous Asphalt in Belgium." Transportation Research Record No. 1265, Porous Asphalt Pavements: An International Perspective 1990, Transportation Research Board, Washington, D.C., 34-40.

Weyrauch, P., Matzinger, A., Pawlowsky-Reusing, E., Plume, S., von Seggern, D., Heinzmann, B., Schroeder, K., and Rouault, P. (2010). "Contribution of combined sewer overflows to trace contaminant loads in urban streams." Water Research, 44(15), 4451-4462.

Whipple, W. (1983). Stormwater Management In Urbanizing Areas, Prentice-Hall.

Winter, T. C., Harvey, J. W., Franke, O. L., and Alley, W. M. (1998). "Ground Water and Surface Water: A Single Resource." Circular: 1139, U.S. Geological Survey (USGS), Denver, CO. 


\section{APPENDIX: SENSOR NAMING}

As mentioned in Section 3.3.1, a nine-character naming scheme was used to identify each sensor's type, location, and measurement. The naming scheme used is as follows: City (1 letter) - Basin Number (1 number) - Control Type (1 letter) - Data Logger Number (1 letter) - Control Associated with Respective Data logger (1 number) - Sensor Type (1 letter) - Unique Name (2 letters) - Measurement Type (1 letter). Table 37 describes each character. The type of measurements from each sensor are explained in Table 38. Unique universal names are provided in look up table 39. In this table parameter $\mathrm{X}$ is

measured from Upgradient Edge, parameter $\mathrm{Y}$ from Curbside, parameter $\mathrm{Z}$ from the surface of the pavement (street level). 
Table 37 - Descrpition of Characters Used in Naming Scheme for Controls 19G \&19H

\begin{tabular}{|c|c|c|}
\hline Descriptor & $\begin{array}{l}\text { Order } \\
\text { (Letters/Numbers) }\end{array}$ & Key \\
\hline City Name & $1^{\text {st }}($ letter $)$ & $\mathrm{L}=$ Louisville \\
\hline Basin number & $2^{\text {nd }}$ (number/letter) & $\begin{array}{l}\text { Basin number within each city. For } \\
\text { Louisville, KY, } 1 \text { = CSO130 }\end{array}$ \\
\hline Control Type & $3^{\text {rd }}$ (letter) & $\begin{array}{l}\mathrm{T}=\text { tree box }, \mathrm{P}=\text { permeable pavement }, \mathrm{B}= \\
\text { bioretention, } \mathrm{G}=\text { green roof, } \mathrm{I}=\text { infiltration } \\
\text { trench, } \mathrm{U}=\text { Dry Well }\end{array}$ \\
\hline Data logger Number & $4^{\text {th }}$ (letter) & $\begin{array}{l}\text { Label of data logger within the basin as A- } \\
\mathrm{Z} \text { (for control 19G: "B", and for control } \\
\text { 19H: "C" }\end{array}$ \\
\hline $\begin{array}{l}\text { Control Associated with } \\
\text { Previous Data logger }\end{array}$ & $5^{\text {th }}$ (number) & $\begin{array}{l}\text { For each data logger, number the controls } \\
1-9 . \text { For both controls } 19 \mathrm{G} \text { and } 19 \mathrm{H}=1\end{array}$ \\
\hline Sensor Type & $6^{\text {th }}$ (letter) & $\begin{array}{l}\mathrm{C}=\text { smart charge controller (power } \\
\text { regulator), } \mathrm{D}=\text { data logger, } \mathrm{H}=\text { humidity } \\
\text { sensor, }, \mathrm{M}=\text { thermistor, } \mathrm{P}=\text { pressure } \\
\text { transducer/piezometer, } \mathrm{T}=\text { time domain } \\
\text { reflectometers (TDR) }\end{array}$ \\
\hline Unique Universal Name & $7^{\text {th }}$ and $8^{\text {th }}$ (letter/number) & $\begin{array}{l}\text { Each sensor will be assigned by a unique } \\
\text { two-digit number, see Table } 38\end{array}$ \\
\hline Measurement Type & $9^{\text {th }}$ (letter/number) & $\begin{array}{l}\text { Indicator of the measured parameter by the } \\
\text { instrument; } \mathrm{A}=\text { voltage ratio (unitless), } \mathrm{B}= \\
\text { battery voltage }(\mathrm{V}), \mathrm{C}=\text { battery current }(\mathrm{A}), \\
\mathrm{D}=\text { drain voltage }(\mathrm{mV}), \mathrm{E}=\text { bulk electrical } \\
\text { conductivity }(\mathrm{dS} / \mathrm{m}), \mathrm{F}=\text { flush count }(\#), \mathrm{H} \\
=\text { humidity }(\%), \mathrm{K}=\text { bulk dielectic } \\
\text { permihermttivity (unitless), } \mathrm{L}=\text { water } \\
\text { level/pressure }(\mathrm{m}), \mathrm{P}=\text { period }(\mathrm{uSec}), \mathrm{R}= \\
\text { rainfall }(\mathrm{mm} \text { per constant time interval), } \mathrm{T} \\
=\text { temperature }\left({ }^{\circ} \mathrm{C}\right), \mathrm{V}=\text { volume }(\mathrm{mL}), \mathrm{W}= \\
\text { volumetric water content }\left(\mathrm{m}^{3} / \mathrm{m}^{3}\right), 1=\mathrm{load} \\
\text { current }(\mathrm{A}), 2=\text { charge input voltage }(\mathrm{V}), 3 \\
=\text { charge input current }(\mathrm{A}), 4=\text { charge state } \\
{[-1=\text { regulator fault, } 0=\text { no charge, } 1=} \\
\text { current limited charging, } 2=\text { cycle } \\
\text { charging, } 3=\text { float charging, } 4=\text { battery } \\
\text { test }], 5=\text { charge source }[0=\text { none, } 1= \\
\text { solar, } 2=\text { continuous }], 6=\text { check battery }[0 \\
=\text { normal, } 1=\text { check battery }]\end{array}$ \\
\hline Additional Information & $10^{\text {th }}$ (letter) & $\begin{array}{l}\text { A way to include additional information } \\
\text { (i.e., } \mathrm{D}=\text { duplicate sensor, or label sensor } \\
\text { manufacturer) }\end{array}$ \\
\hline
\end{tabular}




\begin{tabular}{|c|c|}
\hline Sensor & $\begin{array}{l}\text { Types of Measurements Based on } \\
\text { Abbreviations Described in Table } 6\end{array}$ \\
\hline Smart Charge Controller (Power Regulator) & $\mathrm{B}, \mathrm{C}, 0,1,2,3,4,5, \& 6$ \\
\hline Data logger & $\begin{array}{l}\text { B \& T (stores measurements from all other } \\
\text { sensors) }\end{array}$ \\
\hline Humidity Sensor & 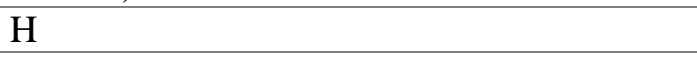 \\
\hline Thermistor & $\mathrm{T}$ \\
\hline Pressure Transducer (Piezometer) & $\mathrm{L} \& \mathrm{~T}$ \\
\hline Time Domain Reflectometer (TDR) & $\mathrm{A}, \mathrm{E}, \mathrm{K}, \mathrm{P}, \mathrm{T}, \& \mathrm{~W}$ \\
\hline
\end{tabular}

Table 39 - Reference Table for Two-Character Unique Naming

\begin{tabular}{|c|c|c|c|c|c|}
\hline $\begin{array}{l}\text { Type of } \\
\text { Sensor }\end{array}$ & Description of the Location & $\underset{(\mathbf{f t})}{\mathrm{X}}$ & $\underset{(\mathbf{f t})}{\mathbf{Y}}$ & $\begin{array}{c}\mathbf{Z} \\
(\mathbf{f t})\end{array}$ & $\begin{array}{c}6^{\text {th }}, 7^{\text {th }}, \text { and } 8^{\text {th }} \\
\text { Characters }\end{array}$ \\
\hline Thermistor & In the Paver & 2.5 & 4 & 0.17 & M01 \\
\hline Thermistor & In the Paver & 40 & 4 & 0.17 & M02 \\
\hline Thermistor & In the Paver & 75 & 4 & 0.17 & M03 \\
\hline Thermistor & 4-ft into the trench & $2.5^{*}$ & 5.5 & 6.5 & M06 \\
\hline Thermistor & 4-ft into the trench & 40 & 5.5 & 6.5 & M07 \\
\hline Thermistor & 4-ft into the trench & 75 & 5.5 & 6.5 & M08 \\
\hline TDR & Bottom of the \#57 gravel layer & 2.5 & 0.5 & 1.4 & T01 \\
\hline TDR & Bottom of the trench & $2.5^{*}$ & 4 & 12.5 & T33 \\
\hline TDR & Bottom of the $\# 57$ gravel layer & 2.5 & 4 & 1.4 & T03 \\
\hline TDR & Bottom of the \#57 gravel layer & 7.5 & 4 & 1.4 & 13 \\
\hline TDR & Bottom of the $\# 57$ gravel layer & 7.5 & 7.5 & 1.4 & 14 \\
\hline TDR & Bottom of the \#57 gravel layer & 20 & 0.5 & 1.4 & 15 \\
\hline TDR & Bottom of the \#57 gravel layer & 20 & 4 & 1.4 & 16 \\
\hline TDR & Bottom of the trench & $1 / 3$ of $L^{* *}$ & 4 & 12.5 & T34 \\
\hline TDR & Bottom of the \#57 gravel layer & 20 & 7.5 & 1.4 & 18 \\
\hline TDR & Bottom of the \#57 gravel layer & 40 & 0.5 & 1.4 & 19 \\
\hline TDR & Bottom of the \#57 gravel layer & 40 & 4 & 1.4 & 20 \\
\hline TDR & Bottom of the trench & $2 / 3$ of $L^{* *}$ & 4 & 12.5 & T35 \\
\hline TDR & Bottom of the \#57 gravel layer & 40 & 7.5 & 1.4 & 22 \\
\hline TDR & Bottom of the \#57 gravel layer & 80 & 4 & 1.4 & 23 \\
\hline TDR & Bottom of the \#57 gravel layer & 80 & 7.5 & 1.4 & 24 \\
\hline TDR & Bottom of the \#57 gravel layer & 120 & 4 & 1.4 & 26 \\
\hline TDR & Bottom of the \#57 gravel layer & 120 & 7.5 & 1.4 & 27 \\
\hline $\begin{array}{c}\text { Pressure } \\
\text { Transducer }\end{array}$ & Bottom of the trench & $2.5^{*}$ & 4 & 12.5 & P40 \\
\hline $\begin{array}{c}\text { Pressure } \\
\text { Transducer }\end{array}$ & Bottom of the trench & $1 / 3$ of $L^{* *}$ & 4 & 12.5 & P41 \\
\hline $\begin{array}{c}\text { Pressure } \\
\text { Transducer }\end{array}$ & Bottom of the trench & $2 / 3$ of $L^{* *}$ & 4 & 12.5 & P42 \\
\hline $\begin{array}{c}\text { Pressure } \\
\text { Transducer }\end{array}$ & Weir box & - & - & - & P99 \\
\hline
\end{tabular}


* In control $19 \mathrm{H}$, the piezometer cluster located at 2.5-ft from the upgradient edge was moved to 4.5 feet because the slope of the trench at the upgradient edge prevented installation as planned. This slope prevented the TDR tines from lying flat.

** "L" stands for the length of the GI control (120 feet for control 19G and 55 feet for control 19H). In 19G, P41 (and T34), and P42 (and T35) are at 40, and 75-ft from the upgradient edge, respectively. In 19H, P41 (and T34), and P42 (and T36) are at 20, and 39$\mathrm{ft}$ from the upgradient edge, respectively. 


\section{APPENDIX: DETERMINATION OF ATTACHED SOLIDS}

The following explains the procedure used by the EPA for determining the

percentage of attached solids of \#3 and \#57 stones, which were used in storage layers of controls $19 \mathrm{G}$ and $19 \mathrm{H}$. The results from this testing were provided in Tables 5 and 6 , see Chapter 3. The data sheet for recording the test results is provided in Figure 103.

\section{Equipment, Materials and Reagents}

1.1. Balance $0.1 \mathrm{~g}$ to $20,000 . \mathrm{g}$

1.1.1. Resolution $0.1 \mathrm{~g}$

1.1.2. Repeatability STD. DEV. 0.1g

1.1.3. Pan Dimensions sufficient to support the sample containers to be used.

1.1.4. Calibration must be verified and recorded daily or as used per manufacturer specification using Class $\mathrm{F}$ weights. If the balance fails the calibration verification the balance must be recalibrated.

1.1.5. Annual Balance Calibration Recertification and maintenance is highly recommended.

1.1.6. Annual Weight Recertification/Recalibration is highly recommended

1.2. Analytical Balance $0.0001 \mathrm{~g}$ to $120 \mathrm{~g}$

1.2.1. Resolution $0.0001 \mathrm{~g}$ 


\subsubsection{Repeatability Linearity $\pm 0.2 \mathrm{mg}$}

1.2.3. Calibration must be verified and recorded daily or as used per manufacturer specification using Class 1 weights. If the balance fails the calibration verification the balance must be recalibrated.Annual Balance Calibration Recertification and maintenance is highly recommended.

1.2.4. Annual Weight Recertification/Recalibration is highly recommended

1.3. Aluminum weigh dishes 4 ”W X 5/16”H

1.4. Whatman 934-AH glass fiber filters $90 \mathrm{~mm}$ diameter or equivalent

1.5. Vacuum Source

1.6. Side arm vacuum flasks (2) 4L Kontes \# 953760-4002 or equivalent

1.7. Bubble Tubing Oxford \#8889-224054 or equivalent

1.8. Filtration Apparatus 1L, $90 \mathrm{~mm}$ (filter funnel, fritted (40 to $60 \mu \mathrm{m}$ frit) glass support base silicone stopper and Al clamp) Wilmed Labglass \# BP-1755-090 or equivalent.

1.9. Desiccator and desiccant

1.10. Drying Oven, gravity-convection type, for operation at $104^{\circ} \mathrm{C} \pm 2^{\circ} \mathrm{C}$ with NIST traceable certified thermometer.

1.11. Refrigerator for use at $4^{\circ} \mathrm{C} \pm 2{ }^{\circ} \mathrm{C}$ with NIST traceable certified thermometer. Temperature of the refrigerator should be recorded at the beginning of each work day on a refrigerator log sheet. The refrigerator should be adjusted as needed to maintain the specified temperature.

1.12. Wash bottles $1 \mathrm{~L}$

\subsection{Type 3 Reagent Grade}

1.14. Sealable pails or shallow tubs. Pails or tubs must be sealable to prevent spilling. Pails or tubs must have rounded corners/seams to prevent entrapment of solids. Pails 
or tubs must be designed for easy transfer of water and solids to pre-cleaned 2-L HDPE wide mouth containers.

1.15. HDPE wide mouth containers 2-L pre-cleaned by EPA procedure B. NOTE: If analysis of samples other than solids is required the appropriate EPA cleaning procedure should be used.

1.16. HDPE pails 19L (5 gallon) with sealable lids pre-cleaned by EPA procedure B. NOTE: If analysis of samples other than solids is required the appropriate EPA cleaning procedure should be used.

1.17. Round tipped forceps

1.18. Plastic bristle brush

1.19. Graduated cylinders $1 \mathrm{~L}, 500 \mathrm{~mL}$ and $100 \mathrm{~mL}$. Class A or B, Calibrated TD.

1.20. Laboratory notebook, bound and paginated.

1.21. Permanent markers, felt tipped.

1.22. Pens with permanent ink.

1.23. Class $F$ weight set, sufficient to verify calibration and recalibrate High Capacity Balance

1.24. Class 1 weight set, sufficient to verify calibration and recalibrate analytical balance

\section{Sample Analysis Procedure}

2.1. Preparation of glass fiber filters

2.1.1. Using forceps place filter wrinkled side up on the filtration apparatus. Apply vacuum and wash the filter with three successive $100 \mathrm{~mL}$ portions of reagent grade water. Continue the vacuum until all visible traces of water have been removed from the filter. Turn off vacuum. Remove filter from the filter apparatus with forceps and transfer to an aluminum weighing dish. Discard wash water. 
2.1.2. Place the aluminum dish with the washed filter into the oven at $104^{\circ} \mathrm{C} \pm 2^{\circ} \mathrm{C}$. Bake for one hour. NOTE: Placing additional washed filters in the oven during the drying will increase the humidity in the oven and extend the drying time.)

5.1.1. Remove from oven and place in desiccator to cool.

5.1.2. Once cool label the dish with a unique ID with a permanent marker, place on the analytical balance, and wait for a stable reading and record the weight of the dried dish and filter. Note: Filters should be weighed immediately after cooling in the dessiccator. Washed filters stored for longer than two days should be re-dried before weighing. Also, marking the dish must be done before weighing. Forceps must be used to handle weigh dishes and filters.

5.2. Record the sample identification of the sample to be processed.

5.3. Place sample bucket on the pan of the high capacity balance. Record the total weight of the sample and container (with lid).

5.4. Prepare four wash containers (Refer to 4.1.4) with sufficient reagent grade water to submerge a piece of the \#3 aggregate. The total volume must not exceed 1.5 L.

5.5. Open the sample container and select one piece of aggregate. Taking care not to lose any solids rinse the loose solids in the water of the first wash pail. Use the plastic bristle brush as needed to remove the fines, frequently rinsing in the first wash container. Continue until the majority of the fines have been removed from the aggregate and captured in the first wash container. Rinse the brush as best possible in the first wash container.

5.6. Transfer the aggregate piece and brush to the second wash container. Repeat the rinsing and washing procedure as described in 5.5. 
5.7. Transfer the aggregate piece and brush to wash container three. Repeat the rinsing and washing procedure as described in 5.5.

5.8. Repeat the process with sufficient wash containers to remove all the fines from the aggregate. Place the washed aggregate into a separate labeled container for storage. Repeat steps 5.5 through 5.7 with each piece of aggregate in the sample.

5.9. The last (in series) wash container water should remain visibly clean until wash containers one through three become too loaded to remove solids. When this container begins to become noticeable cloudy. The first wash container will be emptied into a properly labeled pre-cleaned 2-L HDPE wide mouth containers taking care to not lose any of the solids. Once the bulk of the water and solids has been transferred to the storage container use a wash bottle to rinse and transfer any remaining solids from the wash container. Refill the wash container as described in 5.4. Place this container as the last container in the wash container sequence. Repeat as need until all aggregate sample pieces have been washed.

5.10. After all the pieces of aggregate have been cleaned, rinse the solids from the lid of the original sample pail into the pail using a wash bottle. Rinse the solids from the original sample pail into the first wash container in sequence ensuring all of the solids are transferred. Dry the pail and lid. Place the pail and lid on the pan of the high capacity balance. Record the weight of the empty container.

5.11. Transfer the contents of each of the wash containers to a properly labeled pre-cleaned 2-L HDPE wide mouth container as described in 5.9.

5.12. Place all storage containers in a refrigerator at $4{ }^{\circ} \mathrm{C} \pm 2^{\circ} \mathrm{C}$ for a minimum of 48 hours (If the samples contain mostly large heavy fines and the samples do not cause the 
filters to plug during the filtration step this time may be reduced to 24 or 12 hours). All refrigerated samples must be filtered within three weeks of generation.

5.13. Remove stored samples to be filtered from the refrigerator taking care not to disturb the solids that have settled to the bottom of the container.

5.14. Assemble the filtration apparatus and install a prepared, weighed filter ensuring the wrinkled side is face up. Start the vacuum. Decant slowly from the first storage container of the sample set into the filter funnel apparatus. Take care to avoid disturbing the settled solids on the bottom of the container. Monitor the passage of the supernatant flow through the filter for signs of filter clogging. Do not overload the filter. Ensure that all supernatant passes through the filter within 15 to 30 seconds. If standing supernatant takes longer allow existing supernatant in the filter funnel to pass through the filter. Do not add more supernatant. Rinse the filter funnel, filter and residue with three aliquots of $100 \mathrm{~mL}$ of reagent grade water. If solids are still on the filter funnel or are against the bottom edge of the filter funnel where it meets the filter use the wash bottle to rinse the solids onto the filter and away from the edges toward the center of the filter. If the supernatant does not clog the filter the supernatant from multiple storage containers from the same sample set may be passed through a filter. When decanting leave a small amount of supernatant in the container with the solids.

5.15. Continue the vacuum until all visible signs of water have passed through the filter. Then stop the vacuum. Remove the filter using forceps and place in the weigh dish from which it was removed, ensuring solids are not lost in the transfer.

5.16. Place the dish and filter with solids in the oven set at $104^{\circ} \mathrm{C} \pm 2^{\circ} \mathrm{C}$ for a minimum of two hours. Bake the dish until when cooled and weighed the weigh remains constant. 
Baking times will vary by the oven loading and the amount of solids on the filters. Heavily loaded filters may need to be baked overnight.

5.17. When a constant weight is measured. Record the weight.

5.18. Repeat steps 5.14 through 5.17 as many times as is required to filter all the supernatant from all the storage containers from a single set.

5.19. After all the supernatant has been filtered, setup the filtration apparatus as described in 5.14. With the vacuum on, swirl the container to suspend some of the solids in the small amount of supernatant left in the container. Slowly transfer the remaining contents of the container to the filter ensuring the filter does not become clogged. Using the wash bottle and small amounts of reagent grade water may be added to the solids in the container to aid in transfer to the filter. After a majority of the solids have been transferred to the filter rinse all remaining solids from the container onto the filter. Add $100 \mathrm{~mL}$ of reagent grade water to the sample container, cap, shake vigorously and quickly uncap and transfer to the filter. Repeat this step with two additional aliquots of $100 \mathrm{~mL}$ of reagent grade water. Rinse the filter with solids with three successive aliquots of $100 \mathrm{~mL}$ reagent grade water. Even though the filter may not become clogged with large grit the analyst should limit the loading of the filter to what can be practically transfer to the dish without loss of solids. If solids are still on the filter funnel or are against the bottom edge of the filter funnel where it meets the filter use the wash bottle to rinse the solids onto the filter and away from the edges toward the center of the filter. Settled solids from containers within the same sample set may be added to the same filter as long as the filter does not clog and the solids remain manageable for transfer to the dish. 
5.20. Continue the vacuum until all visible signs of water have passed through the filter. Then stop the vacuum. Remove the filter using forceps and place in the weigh dish from which it was removed, ensuring solids are not lost in the transfer. Use caution when transferring the filter to the dish, mounded, loose, heavy or large grit can easily be dislodged or spilt during transfer.

5.21. Place the dish and filter with solids in the oven set at $104^{\circ} \mathrm{C} \pm 2^{\circ} \mathrm{C}$ for a minimum of four hours. Bake the dish until when cooled and weighed the weigh remains constant. Baking times will vary by the oven loading and the amount of solids on the filters. Heavily loaded filters may need to be baked overnight or longer.

5.22. When a constant weight is measured. Record the weight.

5.23. Repeat steps 5.19 through 5.21 as many times as is required to filter all the settled solids from all the storage containers from a single set.

5.24. Discard the filtrate following site specific disposal methods.

5.25. Ensure the filter apparatus is thoroughly cleaned and rinsed with reagent grade water between different sample sets.

5.26. Recommend the use of LCS (Lab Control Sample)

\section{Calculations}

6.1. weight solids captured on a filter $(\mathrm{mg})=(A-B) \times 1000$

6.1.1. $\mathrm{A}=$ Weight of filter, dish and residue

6.1.2. $\mathrm{B}=$ Weight of filter and dish

6.2. Total Weight of Fines per Sample $=$ Sum of (6.1) per sample set

6.3. Total Aggregate Sample weight $(K g)=(A-B) / 1000$

6.3.1. $\mathrm{A}=$ Weight of the original sample, bucket and lid.

6.3.2. $\mathrm{B}=$ Weight of the bucket and lid empty, clean and dry. 
6.4. Total weight of fines per sample as is $\left(\frac{\mathrm{mg}}{\mathrm{Kg}}\right)=(($ Result of 6.2$) \times 1000) \div$ (Result of 6.3)

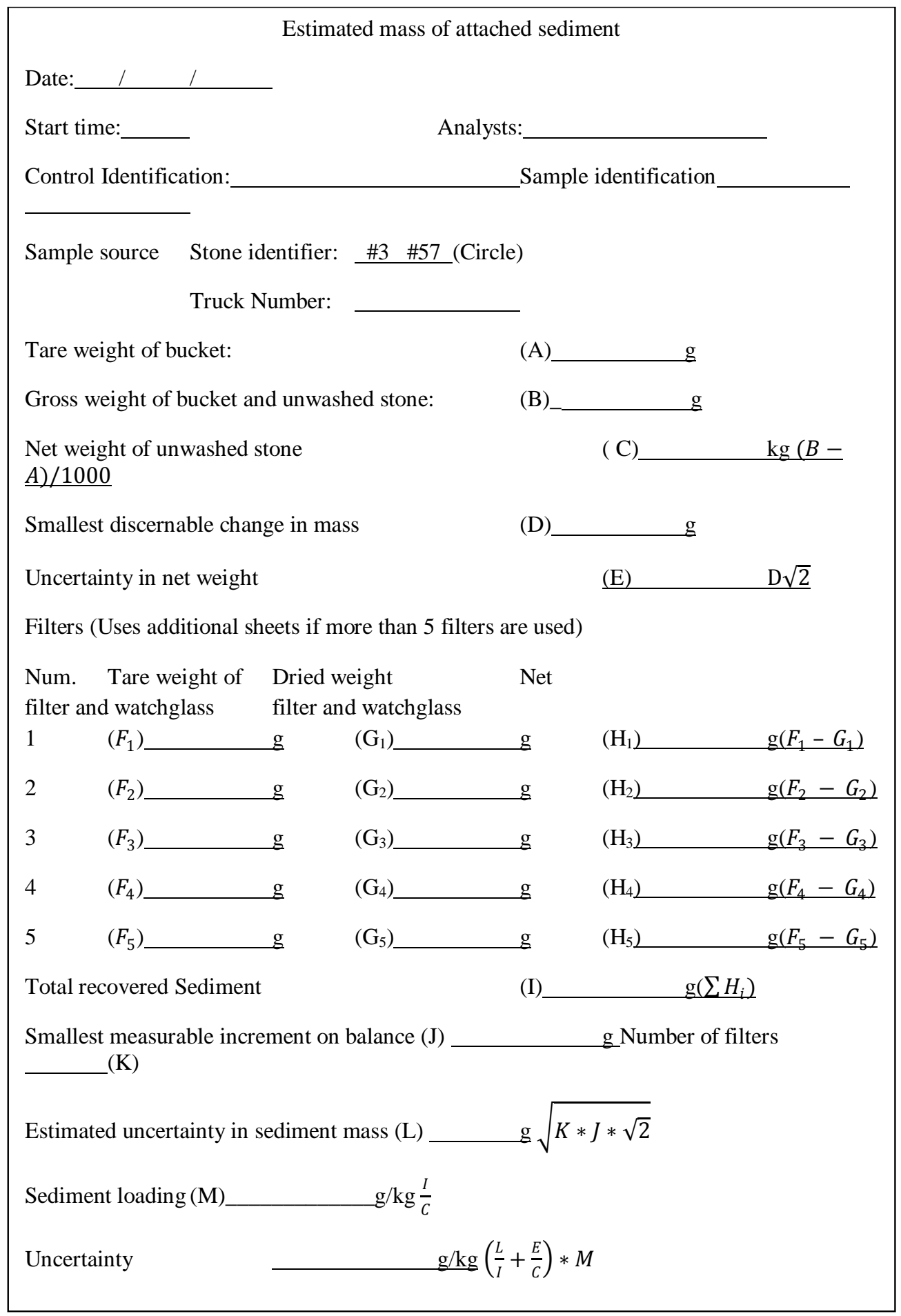

Figure 103 - Data Sheet for Estimating the Sediment Attached to the Aggregate 


\section{APPENDIX: SURFACE INFILTRATION TESTS}

The surface infiltration tests followed a modified version of ASTM method C1701. As explained in Section 3.3.3, the modification was using a neoprene sheeting with combination of weight loads to provide proper sealing of the 12 -in cylinder with pavement, instead of using plumber putty's as originally suggested by the ASTM. The other modification to the ASTM standard, is the requirement of measurement of the water temperature and also the before and after temperatures of the test surface, using a non-

contact thermometer. The modified method was originally developed and used by Borst et al (2010). The test procedure is reviewed here but is also provided in detail by Borst et al (2010).

The 12-in PVC pipe used in this testing is $15 \mathrm{~cm}$ high with parallel black lines drawn 10 and $15 \mathrm{~mm}$ from the bottom of the pipe. As mentioned, a Neoprene sheeting is used to provide sealing with PVC pipe and the permeable pavement surface. The Neoprene sheeting is trimmed to align with the inside circumference of the PVC pipe. A wooden frame which holds four 5-gallon buckets filled with water is used to compress the Neoprene to the pavement surface to provide minimal leakage. Tie-down straps spanning the PVC cylinder support the frame slightly above the permeable pavement surface, see Figure 22. Additional Neoprene strips are placed in the gaps between the individual paver blocks to seal the openings. 
After positioning the pipe and applying the weight, $3.60 \mathrm{~kg}$ of water is poured into the area isolated by the cylinder while making sure the water level stays between the two lines drawn on the bottom interior of the pipe, during the pouring. The time required for the water to infiltrate into the permeable pavement, called the pre-wet time, is measured and recorded. The time begins when the water first contacts the permeable surface and stops when water is no longer visible on the surface. While pouring the water, care must be taken so the water impacts the pavers' surface and not the gap areas. If the pre-wet time is less than 30 seconds, then the infiltration measurement is completed with $18.00 \mathrm{~kg}$ of water. If the time is 30 seconds or more, then the infiltration measurement is made using $3.60 \mathrm{~kg}$ of water.

The temperature of test water and the test area's surface, before and after the experiment is measured using a non-contact thermometer. The temperatures, pre-wet and main tests time, and weight of test water are recorded in special data collection sheets, see Figure 104. The infiltration rate is calculated by use of the following formula: 


\section{Data sheet for modified ASTM C1701 testing}

Date: $1 \quad 1$

Start Time:

Control Identification:

Location Identifier:

Air temp: $\quad{ }^{\circ} \mathrm{C}$

Rain within previous 24 hours? Y / N

Pre-Test surface temperature:

${ }^{\circ} \mathrm{C}$

$\left(\mathrm{T}_{1}\right)$ Pre-wet time:

seconds

Water temp: ${ }^{\circ} \mathrm{C}$

(M) Weight of water used for testing:

If $\mathrm{T}_{1}<\ldots$ seconds then $18.0 \mathrm{~kg}$

if $\mathrm{T}_{1}>$ _ seconds then $3.6 \mathrm{~kg}$

$\left(\mathrm{T}_{2}\right)$ Test time: seconds

Water temp: ${ }^{\circ} \mathrm{C}$

Infiltration rate for 12-inch diameter pipe: $(\mathrm{M} * 458366600) /\left(\mathrm{T}_{2} * 300^{2}\right) \mathrm{cm} / \mathrm{h}$

Post-test surface temp: ${ }^{\circ} \mathrm{C}$

Analysts: $\&$

Photo taken $\mathrm{Y} / \mathrm{N}$ File name:

End time:

Comments:

Figure 104 - Data Collection Sheet for Surface Infiltration Tests 


\section{WATER LEVEL CHANGES IN GI CONTROLS 19G AND 19H}

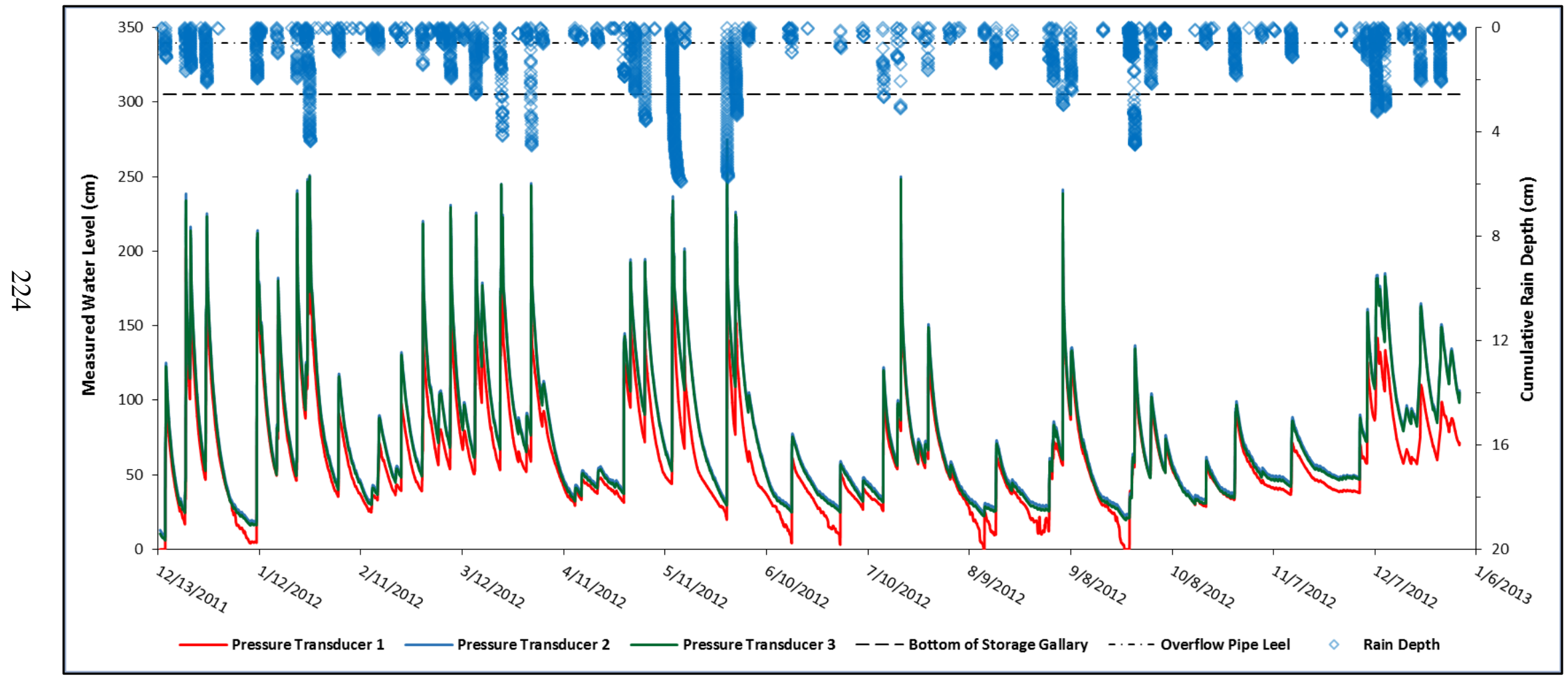

Figure 105 - Changes in Measured Water Level for Control 19G, 2011 \& 2012 


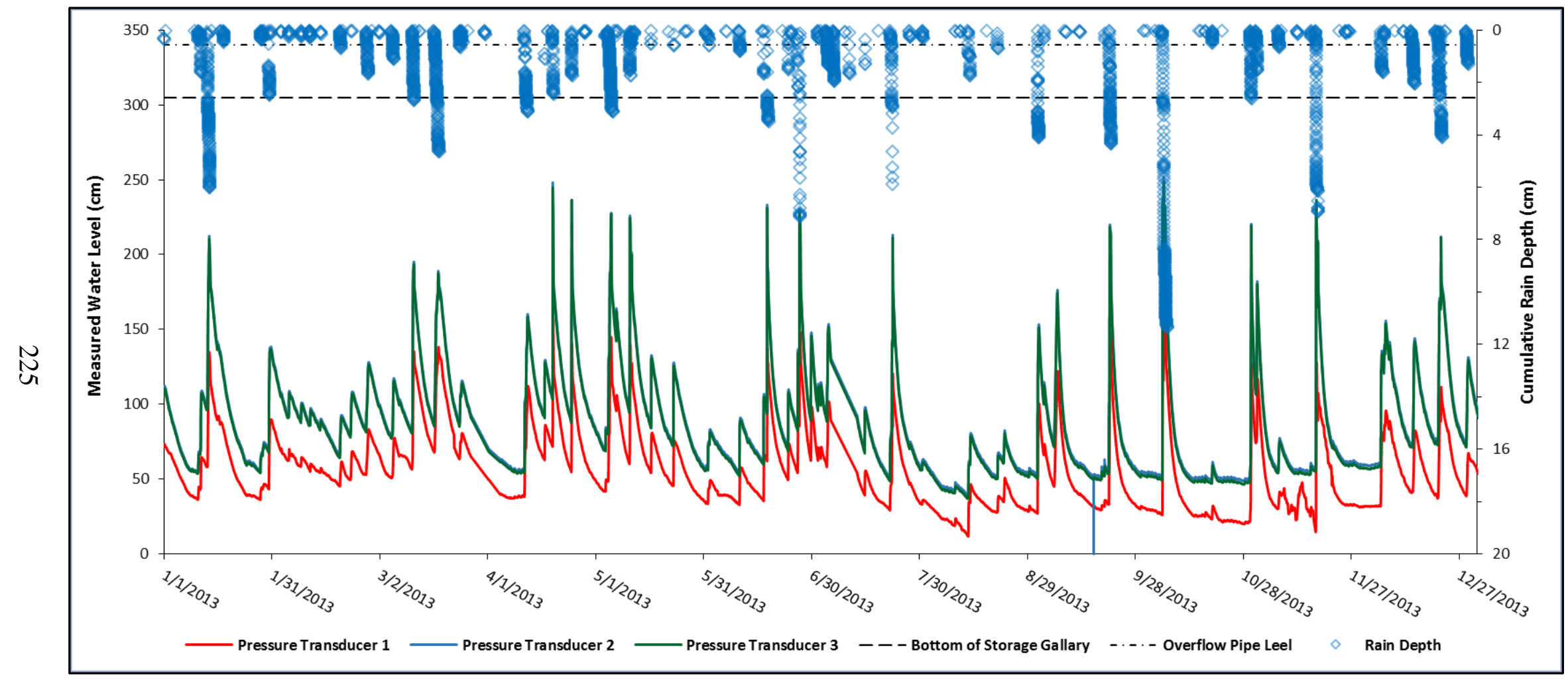

Figure 106 - Changes in Measured Water Level for Control 19G, 2013 


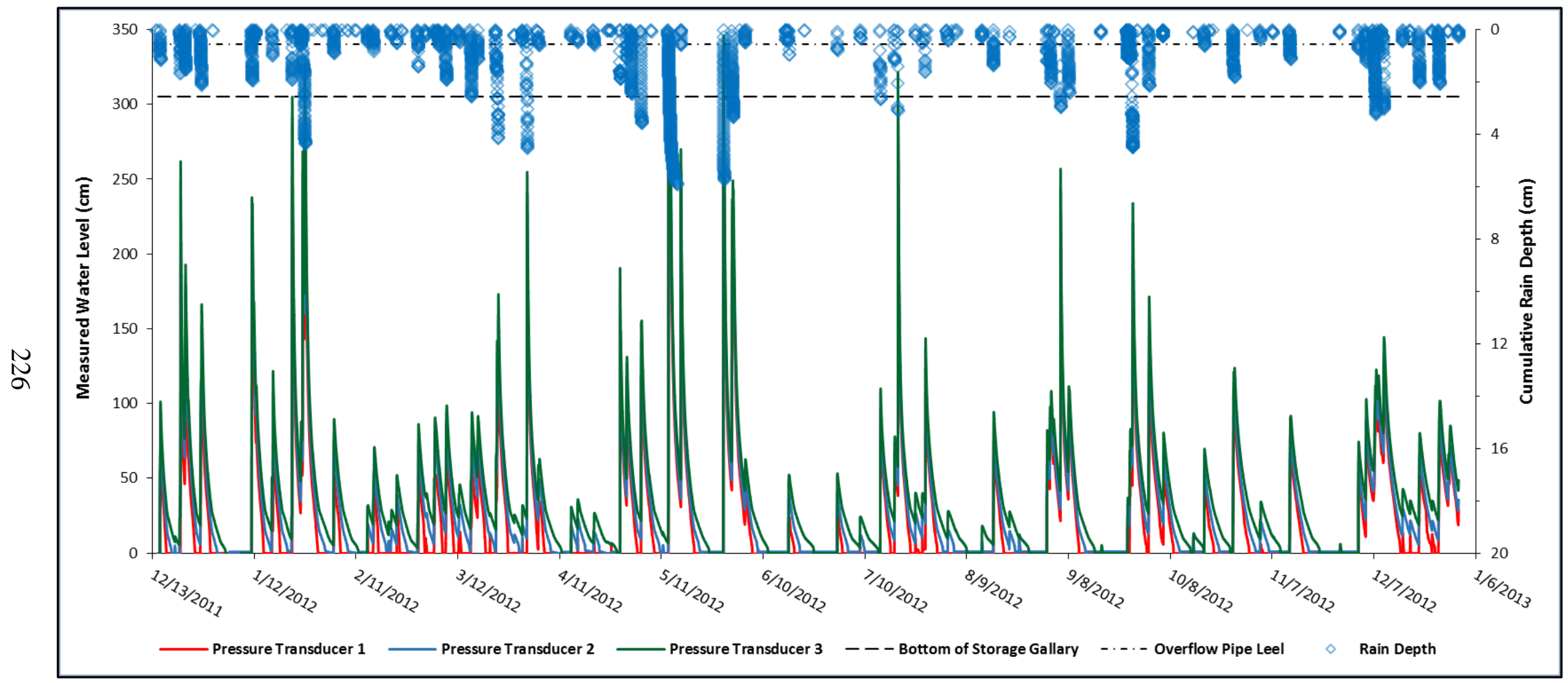

Figure 107 - Changes in Measured Water Level for Control 19H, 2011 \& 2012 


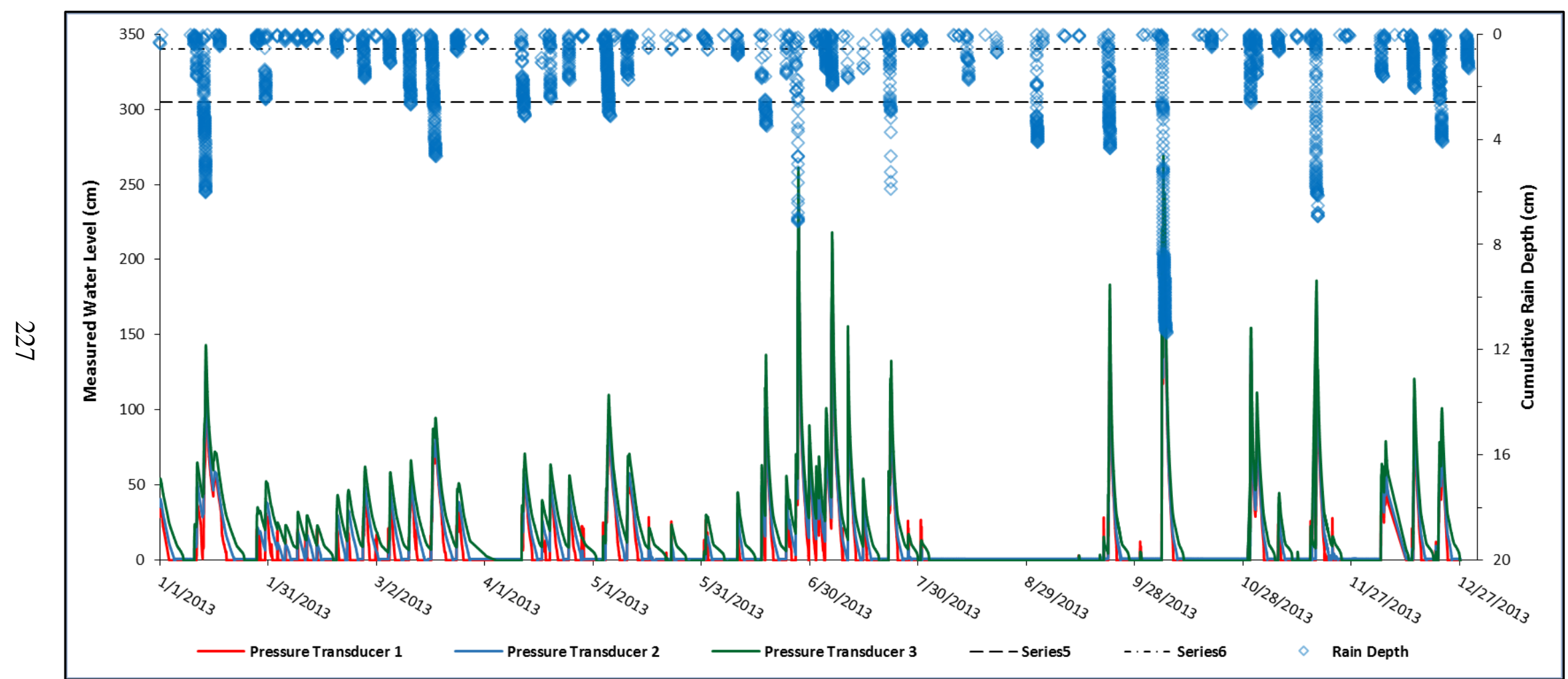

Figure 108 - Changes in Measured Water Level for Control 19H, 2013 


\title{
CURRICULUM VITAE
}

\author{
Hamidreza Kazemi
}

$2301 \mathrm{~S}^{\text {rd }}$ St.

(E.I.T.)

W.S. Speed Hall, CEE Dept.

Louisville, KY 40292

$502 / 852-4617$

\section{Education}

Ph.D. Civil Engineering, December 2014

University of Louisville, Louisville, KY, USA.

- Dissertation: "Evaluating the Effectiveness and Hydrological Performance of Green Infrastructure Stormwater Control Measures"

In this dissertation the long-term hydrological performances and maintenance needs of two permeable pavement systems have been monitored and evaluated through data analysis and modeling techniques. One section of this dissertation also focuses on evaluation the effectiveness of a suite of green infrastructure (GI) stormwater control measures (SCMs) in mitigation of the combined sewer overflow (CSO) issue.

- Coursework: Green Engineering and Sustainable Design, Design of Earth Structures, Earth Pressure and Retaining Structures, Groundwater Hydrology, Non-Destructive Testing, Environmental Analysis Transportation Systems, Rock Mechanics, GPS Theory and Application, Capstone Design

- GPA: 3.9 (coursework)

M.Sc. Civil Engineering- Geotechnical Engineering, April 2010

Ferdowsi University of Mashhad, Mashhad, Iran.

- Thesis: "An Investigation on Consolidation Behavior of Fine-Grained Soils Using Hydraulic Consolidation Cell"

In this thesis changes in horizontal hydraulic conductivity of remolded clays were studied by modeling vertical and radial (inward and outward) drainage paths in hydraulic consolidation cell (Rowe Cell).

- M.Sc. Seminar Lecture: "A Review on Different Slope Stabilization Methods", May 2009 
- Coursework: Advanced Soil Mechanics, Advanced Foundation Engineering, Soil Dynamics, Groundwater Engineering, Advanced Soil Mechanics Laboratory, Earth Dams Engineering, Rock Mechanics, Finite Elements Methods, Advanced Engineering Mathematics

- GPA: 3.25 out of 4

B. Sc. Civil Engineering, September 2006

Ferdowsi University of Mashhad, Mashhad, Iran.

\section{Employment Experience}

Research Assistant, University of Louisville, Louisville, KY, 01/2011 - 11/2014

- "Performance Assessment of Louisville MSD's Integrated Overflow Abatement Plan (IOAP) Individual Projects" 03/2014 - 11/2014

- "Assessing Effectiveness of Green Infrastructure Stormwater Control Measures in CSO Mitigation", CSO130 Project, 01/2014 - 08/2014

- "Evaluation of Hydrological Performance of Permeable Pavement Systems", CSO130 Project, 01/2012 - 11/2014

- "Assessing Maintenance Effectiveness of Permeable Pavement Systems", CSO130 Project, 01/2012 - 12/2013

- Supervised laboratory tests to evaluate the physical properties (porosity, attached solids, organic matter, size distribution) of aggregates and samples collected from CSO130 project, 08/2012 - 10/2013

- Assisted the Installation of Electronic Sensors for Monitoring the Performance of Green Infrastructure Stormwater Control Measures (four permeable pavement systems and six treeboxes), $2^{\text {nd }}$ Phase of CSO130 Green Practices Installation, 02/2013 - 04/2013

- Assisted the Installation of Electronic Sensors for Monitoring the Performance of Green Infrastructure Stormwater Control Measures (two permeable pavement systems), $1^{\text {st }}$ Phase of CSO130 Green Practices Installation, 11/2011 - 12/2011

- Research on Hydrological Performance of Green Infrastructure (GI) Stormwater Control Measures (SCMs), 01/2011 - 11/2011

Visiting Researcher, Environmental Protection Agency, Office of Research and Development, Edison, NJ, 2011 - 2012

- Data Analysis on Monitoring Data Collected from CSO130 Project, 01/2012 $07 / 2012$

- Conducted laboratory tests to investigate the performance and issues observed with some of the electronic sensors used in monitoring of CSO130 project, $03 / 2012-06 / 2012$

- Assisted during the Development of the Addendum to Quality Assurance Project Plan (QAPP) for $2^{\text {nd }}$ Phase of CSO130 Project 01/2012 - 07/2012 
- Assisted during the Development of the Quality Assurance Project Plan (QAPP) for $1^{\text {st }}$ Phase of CSO130 Project 05/2010 - 08/2010

- Research on Performance of Green Infrastructure (GI) Stormwater Control Measures (SCMs)

Teaching Assistant, Ferdowsi University of Mashhad, Mashhad, Iran. 09/2007 - 06/2009

- Foundation Engineering, 4 Semesters. (09/2007-06/2009)

- Soil Mechanics Laboratory, (Spring Semester 2009)

- Advanced Soil Mechanics, (Fall Semester 2008)

- Soil Mechanics, 2 Semesters. (Sep. 2007-June 2008)

Tutor, Mashhad, Iran 2006-2009

- More than 200 hours of tutoring, Soil Mechanics and Foundation Engineering

Supervisor, Computer Lab of Civil Engineering Graduate Students, Ferdowsi University, Mashhad, Iran, 09/2006 - 12/2007

Co-Op, Technical \& Soil Mechanics Laboratory Company, Iran Ministry of Road and Transportation, Mashhad Branch, Mashhad, Iran, 06/2006 - 09/2006

\section{Publications/Presentations}

Publications

- Kazemi, H., Rockaway, T.D., and Rivard, J.A., (2013) "Monitoring Hydrological Performance of Green Infrastructure Stormwater Controls" ASCE-EWRI World Environmental and Water Resource Congress Cincinnati, Ohio May 19-23

- Kazemi, H., and Bolouri-Bazaz J., "A review On Consolidation Behavior and determining Horizontal Hydraulic Conductivity of Fine Grained Soils by Using Hydraulic Consolidation Cell", 5th National Congress on Civil Engineering, Mashhad, Iran, May 2010.

Presentations

- Kazemi, H., Rivard, J.A, Rockaway, T.D., (2014) "Performance of Stormwater Control Measures: Challenges and Lessons Learned" Kentucky Society of Professional Engineers Annual Conference, Owensboro, KY June 26

- Kazemi, H., Rockaway, T.D., and Rivard, J.A., (2014) "Hydrological Performance of Two Permeable Pavement Storm-Water Control Measures in Louisville, Kentucky" ASCE-EWRI World Environmental and Water Resource Congress, Portland, Oregon June 1-5

- Kazemi, H., Rockaway, T.D., and Rivard, J.A., (2014) "Assessment of Infiltration Performance and Maintenance of PaveDrain pavements" PaveDrain(C's Annual Sales Meeting, Baltimore, Maryland January 7-9

- Rivard, J.A, Kazemi, H., and Rockaway, T.D., (2013) "Assessment of Maintenance and Operations for Permeable Pavers" Five Cities Plus Conference, Louisville, KY November 13, 2013 


\section{Publications/Presentations (Continued)}

- Kazemi, H., Rockaway, T.D., and Rivard, J.A., (2013) "Performance of Green Infrastructure Stormwater Controls" Water Professional Conference Green Workshop Louisville, KY September 2013

- Kazemi, H., Rockaway, T.D., and Rivard, J.A., (2013) "Monitoring Hydrological Performance of Green Infrastructure Stormwater Controls" Kentucky Society of Professional Engineers Annual Conference, Louisville, KY April 10

- Kazemi, H., Rockaway, T.D., and Rivard, J.A., (2013) "Monitoring Hydrological Performance of Green Infrastructure Stormwater Controls" ASCE-EWRI World Environmental and Water Resource Congress Cincinnati, Ohio May 19-23

- Ehsaei A. and Kazemi, H., (2012) "Effective monitoring instrumentation of Green stormwater management practices" Kentucky Tennessee AWWA Annual Conference, Memphis, Tennessee July 10.

- Kazemi, H., (2012) "Evaluating the Performance and Behavior of Green Infrastructure Stormwater Control Systems" ASCE Kentucky October 2012.

- Brown, R. A., BORST, M., Kazemi, H., Gray, J., and Kurtz, L., (2012) "Case Study: Installation and Monitoring of Green Infrastructure Stormwater Controls in Louisville, Kentucky" ASCE-EWRI World Environmental and Water Resource Congress Albuquerque, NM, May 20 - 24

Posters:

- Kazemi, H., Rockaway, T.D., and Rivard, J.A., (2014) "Mitigating Combined Sewer Overflows with Green Infrastructure Storm-Water Control Measures in Louisville, Kentucky" ASCE-EWRI World Environmental and Water Resource Congress, Portland, Oregon June 1-5

- Kazemi, H., Rockaway, T.D., and Rivard, J.A., (2013) "Tracking the Hydrological Performance of Permeable Pavement Systems" International Low Impact Development Symposium St. Paul, MN August 18-21

- Kazemi, H. and Zomorrodian, M. A., (2011) "An Overview on University of Louisville's Green Infrastructure Master Planning" UofL Homecoming Reception Louisville, Kentucky October 2011

\section{$\underline{\text { Awards and Honors }}$}

- Recipient of Grosscurth Ph.D. Fellowship which covered 2-years of stipend, full tuition reemission and health insurance, University of Louisville, Louisville, KY, August 2010 - June 2012.

- Member of Golden Key International Honor Society, 2011 - Present 